\title{
U N S S
}

suURUII BXTRAMARINORUIII

\section{SCANDINAVIAE}

(SUECIE, NORVEGIF, DANI \& \& FENNIE).

AUCTORE

$$
381059
$$

CARL AGARDH WESTERLUND.

(Societati exhibitum d. 1 Febr. 1896).

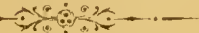

HELSINGFORS 1897. 


$$
1
$$




\section{SYNOPSIS}

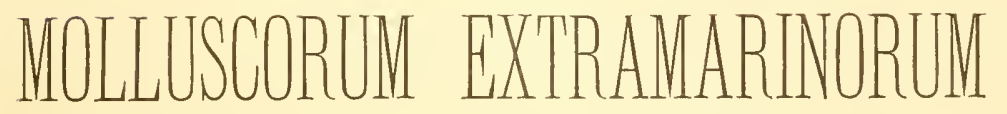

\section{SCPNDINAVI AE}

(SUEGIE, NORVEGIE, DANIA \& FENNIE.)

AUCTORE

CARL AGARDH WESTERLUND.

11)

(Societati exhibitum d. 1 Febr. 1896).

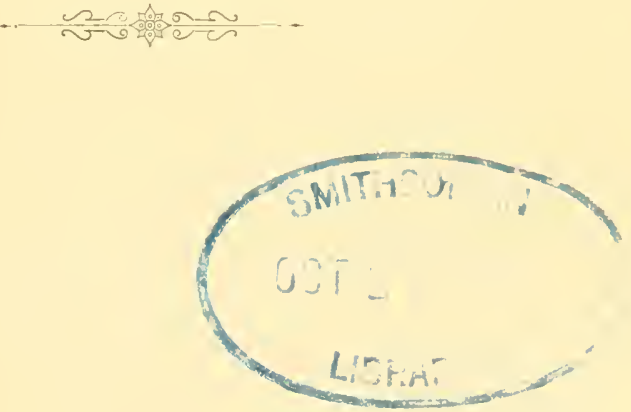





\begin{abstract}
Cum fauna palearctica molluscorum extramarinorum his proximis annis modo critica pertractata sit atque etiam a multis partibus prorsus renovata, quod et quænam ratio inter singulas species ejus intersit nova luce illustratum certioribusque experimentis exploratum est et, id quod ad retractationem illam necessarium est, diligenter est quiesitum vel quo modo et quatenus singula genera cœlo ac temperie terræ dependeant vel quo modo et quatenus ob eam ipsam rem variationem patiantur, propter has res atque etiam propter magnam illam multitudinem novarum formarum, quæ his decem proximis annis etiam in septentrionalibus terris sunt inventx, factum est ut Fauna molluscorum Scandinavixe in permultis ejus generibus longe alia nunc appareat, atque decem abhinc annos erat.

Quæ fauna cum et ipsa sua, ut ita dicam, vetustate et terrarum Scandinavicarum ad boream situ ad totam fuunam molluscorum constituendam magni momenti sit, eam critico modo breviter retractare operæ pretium esse putavi.
\end{abstract}





\section{Fontes hujus opuseuli.}

\section{Scripta edita.}

\section{De molluscis suecanis.}

Carl Lime. Natus 1707, denatus 1778. - Iter Oelandicum 1741, Systema Naturæ ed. 10. 1758, Fauna Suecica ed. 1. 1746, ed. 2, 1761.

In his operibus sequentia mollusca terrestria et fluviatilia Sueciæ descripsit (nomina in parenthesi inclusa sunt cujusque auctoris): Arion (Limax) ater, Limax maximus, Agriolimax (Limax) agrestis, Helix hispida, lapicida, arbustorum, hortensis (nemoralis!), pomatia, Pupa (Turbo) avenacea (muscorum), Balea (Turbo) perversa, Succinea (Helix) putris, Physa (Bulla) fontinalis, hypnorun, Limnata (Helix) stagnatis, auricularia, balthica, Pianorbis (Helix) corneus, umbilicatus (planorbis) albus?, spirorbis. contortus, vortex, crista (in S. N. ed. XII nautileus), complanatus, Ancylus (Patella) lacustris, Paludina (Helix) vivipara, Bythinia (Helix) tentoculata, Neritina (Nerita) Auviatilis cum var. lacustris et litoralis, Sphorium (Tellina) comenu, Anodonta (Mytilus) anatina, Margaritana (Mya) margaritifera. Adhuc descripsit sub nomine Hel. albellee $\mathrm{H}$. lapicidre juv. et s. n. Hel. fragilis juvenem (h. e.superam partem spiræ) varietatis cujus dam Limnææ stagnalis. In Syst. Nat. ed. X Heticem gothicam descripsit (st. carinata, utrinque convexa, cornea fasciis subferrugineis; habitat in Sueciæ nemoribus"), sed postea in Fauna Suec. ed. 2 omisit. Cl. Mörch (Syn. Moll. Dan.) opinat hanc testam Patula rotundata Müll. fuisse.

Helix limosa Lin. etiam est species de qua quæritur. Alii et egomet putavimus, verbis auctoris in Fanna Suec. ed. I („operculo etiam clauditur") ducti, eam Bythiniæ speciem esse. Confiteor tamen eos, qui in hac limosa Lin. Limnceam ovatam Drap. vidunt, causam quandam habere, quod Linné in editione altera suæ Faunæ verba ex editione prima citata forte ut errata omisit; quod numquam in Scandinavia species in fluviis habitans, operculata, "testa pellncida et valde tenera ac acuta, apertura ovata" inventa est, et quod difficile est credere Linneum hanc sub nna alterave forma vulgatissimam speciem non cognovisse.

Observare necesse est, synonyma Linnei ex auctoribus alienis, siepissime tantum ex figuris eorum tunc temporis parum bonis capta, 
rstimatione omnino in judicando specierum ejus carere, in qua re maximus pondus in locis originalibus patriæ jacet. Observes etiam editionem secundam Faunæ Suecire (1761), certissime non ab auctore ipso tandem curatam, multo minus valere quam editio prima (1748), quod variis locis copiose in Fauna molluscorum Suec. etc. (1873) demonstravi.

\section{Johan Fischerström.}

Nat. $28 / 7$ 1735, in juventute literis humanioribus deditus, postea scientias aconomicas studuit et in his scripta plura publicavit; den. in Stockholm 23/11 1796.

- In »Utkast till beskrifning om Mälaren», 1785, p. 236 -245, enumerat mollusca præcipue ab in his rebus bene erudito Adolf Modéer ad et in lacu hujus nominis observata.

Hic $A d$. Modéer fuit nat. 15/4 1739 in Carlskrona, civis acad. in Iund 1754, geometer et ab anno 1770 secretarius societatis Svensha Patriotiska Sällskapet, bonus observator, diligens auctor in historia naturali et præcipue in rebus œconomicis; den. in Stockholm $16 / 71799$.

Anders Johan Hagström nob. Hagströmer.

Nat. 8/9 1753 ad Linna prov. Södermanland, civ. acad. in Upsala, doctor med. 1781 in Åbo, med. prof. 1793 in Stockholm, director summus 1808, nobili nomine ornatus 1812 , botanicus, medicus frequenter exercitatus, prælector præstans, pro benignitate et liberalitate laudatus; den. in Stockholm 8/3 1830.

- In » Weckoskrift för Läkare och Naturforskare», p. 185 -191, indicem molluscorum circa Stockholm cum auxilio jam laudati Ad. Modéer elaboravit.

\section{Anders Jahan Retzius.}

Nat. 3/10 1742 in Kristianstad, discipulus inter optimos Limnéi, docens in Lund 1764, professor hist. natur. et œconomiæ extraord. ibidem 1777, ord. 1781, "in scientiis omnibus giganteus"; den. in Stockholm 6/10 1821.

- Dissertatio Nova Testaceorum Generum sistens. Lundæ 1788.

In hac dissertatione genus Unio creavit et faunam $U$. crasso 11 . sp. \& $U$. tumido n. sp. altgmentavit. (Cfr. C. G. Westerlund in Nachrichtsblatt Mal. Ges. 1889 p. 19-23).

Sven Nilsson.

Nat. $8 / 31787$ in Altvastorp prov. Skåne, ubi pater agricola levis, civ. acad. in Lund 1806, doctor phil. ib. 1811, prof. honor. 1821, 1828-1831 præfector zool. in Mruseo Acad. Scientiarum Hol 
miæ, 1831-1856 prof. zool. ord. in Lund, 1838 etiam pastor et præpositus, zoologus, operibus suis de vertebratis Scandinaviæ meritissimus, iis de molluscis et de vestigiis rerum antiquarum fundator harum scientiarum patriæ; den. in Lund 30/11 1883.

- Historia molluscorum terrestrium et fluviatilium Sueciæe. Lundac 1822.

Maxime hoc opere studium molluscorum, ,inde a temporibus immortalis Linnæi in Suecia plane neglectum", ut ipse dicit, apud nos ampliavit, scientiam observationibus numerosis magni ponderis illustravit et faumam suecicam formis sequentibus auxit: Limax tenellus n. sp., marginatus (cinereus var.), Vitrina pellucida, Conulus (Helix) fulvus, Vitrea (Hx.) crystallina, Zonitoides $(\mathbf{H x}$.$) nitidus, Hyalinia (\mathbf{H x}$.$) cel-$ laria, Arion (Limax) albus, cinctus (fasciatus $\nu, \delta, \varepsilon, \zeta)$, bourguignati (fasciatus $\alpha, \beta, \gamma)$, flavus, Punctum (Hx.) pygmœum, Patula (Hx.) rotundata, ruderata (rotund. var.), Helix pulchella, costata (pulch. var.) aculeata, bidens, strigella, incarnata, fruticum, striata (ericetorum), nemoralis, Buliminus (Bulimus) obscurus, Pupa muscorum, costulata n. sp., antivertigo, pygmae, pusilla (vertigo), Claus. laminata (bidens), plicatula, bidentata (rugosa), nilssoni (papillaris), dubia (rugosa var.), Zua (Bulimus) lubrica, Cecilianella (Achatina) acicula, Succ. pfeifferi (S. amphibia var.), Carychium (Auricula) minimum, Amplipeplea (11. gen.) glutinosa, Limnce ovata, lagotis (ovata var.), prevegra, palustris, var. fusca, succinea n. sp., glabra (elongata), truncatula (minuta), Planorbis albus (hispidus), carinatus, crista (imbricatus), nitidus, Ancylus fluviatilis, Bythinia (Paludina) leachi (sp. sine nom.), Talvata piscinalis, pusilla, cristata, Sphwrium (Cyclas) ovale (Iacustre), calyculatum, Pisid. amnicum (obliquum), subtruncatum (obtusale f. Malm), pusillum (fontinale f. Malm), Unic ater n. sp., rostratus (pictorum), batavus, limosus n. sp., Margaritana (Unio) elongata n. sp., Anodonta sulcata et piscinalis n. sp. Species, quam ut "Helicem conspurcatam" descripsit, est H. strigella juvenis, Palud. balthica et octona ad faunam marinam pertinent. Anod. anatina $\alpha$ est forma nunc indeterminabilis et A. intermedia "Pfr." verosimiliter A. cygnæea immatura, ut docet cel. Rossmässler.

Johan Emanuel Wikström.

Nat. 1/11 1789 in Wänersborg, civ. acad. in Upsala 1806, doctor medic. 1817, botan. professor ad. Museum Reg. Acad. Scient. in Stockholm, botanicus eruditus; den. $4 / 51856$.

- In introductione suxe »Stockholms Flora», I. 1840, p. 118-131, bonam faunulam molluscorum regionis circa Stockholm dedit.

D. S. Högberg.

- Om några för Sverige nya arter och formförändringar af Land- och Insjösnäckor »in K. Sv. Vet. Ak. Handl. 1841 p. 
197-205̃. Idem etiam distribuavit »Typsammling för Srenska snïckor.»

Primus Pupam arenaceam, cylindraceam, edentulam et Succ. oblongam recte in Suecia distinxit.

\section{Carl Henrik Boheman.}

Nat. 10/7 1796 in Jönköping, a patre ad mercatorem destinatus, postea ipse vitæ genus mutavit, juris stud. in Lund 1812, tamen 1813 dux militaris inferior et 1814 particeps in expeditione bellica ad Norregiam, centurio 1837; in omnibus his annis studia insectorum ardore coluit, itaque 1841 professorem entomologiæ ad Mus. Reg. Acad. Scient. in Stockholm constitutus est et scripta plura egregia in scientia sua elaboravit; den. in Stockholm $2 / 111868$.

- In Öfversigt af K. Sv. Vet. Ak. Förh. 1844 enumeravit »Land- och Sötvattenmollusker, funna omkring Qvickjock i Luleå Lappmark.»

Helix harpa Say est ab eo in Lapponia detecta.

L. P. Hansén.

- In Öfvers. af K. Sv. Vet. Ak. Förh. 1845 p. $254-25 \overline{7}$ scripsit »Om nya svenska sötvattensmollusker».

— Ibidem 1848 p. 201 -202: Nya svenska snäckor.

Claus. ventricosam, Valv. macrostomam (depressam), Pisid. henslouianum et pulchellum primus in faunam nostram introduxit ut etiam $P\left(a^{-}\right.$ lud. contectam in Suecia primus indicavit. Quid "Anod, rostrata" et .ponderosa“ ejus sint, munc est agerrime judicare.

\section{Withelm Lilljeborg.}

Nat. ${ }^{6} / 101816$ in Helsingborg, civ. acad. in Lund 1834, doctor phil. 1841, prof. zool. extraord. in Lund 1853, prof. zool. ord. in Upsala 185́4-1882, doct. med. honor. 1877; itineribus et excursionibus per vitam longam numerosis faunæ terrarum borealium optime peritus, scriptis zoolog. numerosis celeberrimus, præcipue opere magno monumentali de animalibus vertebratis Sueciæ et Norvegiæ laudatus.

- In Öfvers. af K. Sv. Vet. Ak. Förh. 1858 pp. 13 c. 1 tab. communicavit »Några anmärkningsvärda former af Clausilia rugosa Drap., Rossm., eller Claus. nigricans Mat. and Rackett, Forbes and Hanley, hvilka förut hos oss dels icke blifvit anmirkte och dels blifvit förvexlade med andra.» 
mollusker.

- Ibidem 1850 p. 58-90: Gotlands Land- och Sötvattens-

- Ibidem 1849 et in K. Sv. Vet. Ak. Handl. 1850: Förteckning på land- och sötvattens-blötdjur, funna vid Tromsö i Norge, i Jemtland i Sverige samt i Ryssland.

Helix lamellata, Claus. connectens (rugosa f. major), sejuncta (rugosa г. pumila) et Succ. arenaria ab eo invente sunt in Suecia, et in Norregia Helix harpa, Pupa substr. v. monas et Balea perv. v. rayiana.

August Wilhelm Malm.

Nat. $23 / 71821$ in Lund, civ, acad. ib. 1838, præfector musei in Göteborg 1848, de quo meritissimus, zoologus, 1858 professoris nomine honoratus, observator bonus, collector et descriptor rerum naturalium infatigabilis; den. in Göteborg 5/3 1882.

— In Göteborgs Vet. och Vitt. Samh. Handl. 1851 p. 111 -131 \& 1855 p. 77-152 tractavit »Om Svenska Landt- och Sötvattens-mollusker, med särskildt afseende på de arter och former, som förekomma i granskapet af Christianstad och Göteborg."

-- Ibidem 1863 p. 135-145: Om Limnxa limosa Malm.

- Ibidem 1863: Skandinaviska Land-Sniglar, Limacina, afbildade efter lefvande exemplar och beskrifna. Pp. 93 cum 5 tab. col.

Observationes ejus circa subfam. Limacina et Arionina, fam. Sphreriidce et Unionida nec non genus Limna sollicite factæ et magni scientiæ ponderis sunt, licet conclusiones non semper probandæ sint. Species et varietates plures faunæ novas distinxit, scilicet: Hyalin. hammonis (Hx. pura), Pupa substriata, Plan. riparius (glaber), Hydrobia steini (Byth. similis), Pisidium nitidum, personatum n., milium (arcæforme), obtusale, nee non Hyal. nitens (nitidula), Zua lubr. v. nilssoni n., (Paludina contecta), Valiata antiqua (contorta).

\section{Gustaf Lindström.}

Nat. 27/8 1829 in Visby, civ. acad. in Upsala 1848, doct. phil. ib. 185́, adj. scholæ in Visby 1856, prof. et præf. palæontol. Musei Reg. Acad. Scient. in Stockholm 1876.

- In Öfvers. af K. Vet. Ak. Förh. 1855 p. 49-: Om utvecklingen af Neritina fluviatilis Müll.

— Ibid.: Bidrag till kännedomen om Östersjöns invertebratfauna. 
- In » Visby läroverks skolprogram 1868»: Om Gotlands nutida mollusker, pp. 48 cum 3 tab.

In his operibus palæontologus laudatus obserrationes egregias præsertim anatomicas de rariis molluscis communicarit.

Lars Edvard Walmistedt.

Nat. ${ }^{14}, 101819$ in Cpsala, docens ib. 1849, prof. mineralogixe et geologiæ ib. 1859-1884, successor patris; den. ib. $3 / 2$ 1892.

- In Öfrers. af K. Sr. Vet. Ak. Förh. $185 j$ p. 79-87:

Om slägtet Clausilia och dess i Srerige förekommande arter. Clausilia plicata Drp. ab eo in Suecia (ins. Gotland) detecta.

\section{Hans Darid Johan W'allengren.}

Nat. $8 / 61823$ in Lund, cir. acad. ib. 1842 , sacerdos $184 \bar{i}$, pastor eccles. in Farhult pror. Skåne 1866, zoologus egregius, præcipue scriptis multis entomologicis celebratus; den. ${ }^{25} 101894$.

- Skandinariska arter af slägtet Pupa. Lund 185̄6, pp. 16. Dissertatio academica.

— In Öfvers. af K. V'et. Ak. Förh. 1857 p.118-121: Landoch sötrattens-mollusker i nordöstra Skåne.

Formam Pupce cylindracece edentulam (dilucida Wallengr.) et rar. acronostomam ejusdem speciei (nilssoni n. sp. $\mathrm{Wgr}$.) in Gotlandia detexit.

Carl Friedrich Theodor Gideon de Wallenberg.

Nat. 1836 in rico Gross-Peterwitz prope oppidum Neumarkt Silesiæ, civ. acad. unirers. Tratislariæ 1855, doct. phil. in Berlin 1858 , postea in annis $1859-1860$ studia astronomica et magnetica in Sternwarte in Berlin et eum prof. Lamont in itinere in Gallia et Hispania curarit, tandem, ægrotatione coactus, his studiis absistens, ritam ad finem actnosam in prædio suo equestri Obra provinciæ Posen degit, ubi 30/1 1872 mortuus est.

- De molluscis Lapponire Lulensis. Berolini 1858, pp. 46 c. 1 tab. Dissertatio inauguralis.

Cum obserrationibus bonis de molluscis lapponicis eorumque distributione geographica etiam sequentes novitias faunæ continet dissertatio ejus: Hyal. petronella (viridula), Pupa arctica n. sp., alpestris (shuttleworthiana), gredleri (columella).

Carl August Gosselman.

Nat. ${ }^{18} / 81831$ in Carlskrona, cir. acad. in Lund 18 19 , doct. phil. ib. 185̌3, lector scholie in Carlskrona 186x; den. ${ }^{15} / 41893$. 
- Zoologiska och geologiska iakttagelser inom Blekinge. Lund 1863. Diss. acad. in qua enumerationem molluscorum rariorum hujus provinciæ sibi cognitorum fecit.

\section{Johan Emanuel Zetterstedt.}

Nat. ${ }^{23} / 41828$ in par. Viby prov. Nerike, civ, acad. in Upsala 1846, doct. phil. ib. 1854, docens œeonomiæ practicæ ib. 18ว̄, lector scholæ in Jönköping 1863, botanicus meritus; den 12/2 1880.

- In K. Sv. Vet. Akad. Handl. 1864 manu erudito inventum Bulimini montani in Suecia inopinatum descripsit.

\section{Carl Hartman.}

Nat. $5 / 61824$ in Ulriksdal prope Stockholm. civ. acad. in Upsala 1842, doct. phil. ib. 1848, lector scholæ in Örebro 1S59; botanicus egregius editionibus 6 ultimis semper forteque auctis operis laudati patris "Handbok i Skandinaviens Flora" (ed. I. 1820) studium botanicum patrix maxime aluit; den. in Örebro 1884.

- Spridda bidrag till Nerikes naturalhistoria, Mollusca, 1864, pp. 13 in 4:0. Diss. pro munere lectorali.

- In Öfvers. af K. Sv. Vet. Ak. Förh. 1866 p. 381--394: Land- och Sötvattens-mollusker i östra delen af Stockholmstrakten.

Pupam minutissimam in Suecia detexit.

\section{August Gustav Eisen \& Anton Julius Stuxbery.}

Eisen: nat. $\% 1849$ in Stockholm, civ. acad. in Upsala 18tis, cand. phil. et docens zool. ib. 1873, post itineribus scientificis in variis Europæ terris ad Calitorniam 1873 transmigravit et postea Californiam et varias terras Americæ centralis et borealis perlustravit et scripta multa historiam naturalem tractantia edidit; doctor phil. honor. in Upsala 1893.

Stuxberg: nat. 18/4 1849, civ. acad. in Upsala 1869, doct. phil. ib. 1875, primo itinera scientifica plura in Suecia habuit, publice et privatim, deinde itinera illa arctica laudata duce nostro A. E. Nordenskiöld ut zoologus expeditionum fecit, 1875 ad Novaja Semlja et Jenisej, 1876 ad Jenisej et 1878-1880 cum nave Vega circa Asiam et Europam, quæ itinera in variis operibus deligenter descripsit ut etiam numerosa scripta præcipue zoologica publicavit; ab anno 1882 curator zoologiæ musei in Göteborg. 
- In Öfvers. af K. Sv. Vet. Akad. Förh. 1868, mollusca p. 373-374: Bidrag till kännedomen om Gotska Sandön.

Lim. maximus var. calosoma et $L$. tenellus mut. phoconotus in opusculo novitiæ sunt.

- Stephan Clessin, bavaricus. Laudabile coluit partem de Sphariidreis scandinavicis in mea Fauna Suecix, Norvegixe et Daniæe (1873), nec non scripsit

— Nordschwedische Mollusken» in Malak. Blätter 1878 et ibidem »Nordschwedische Mollusken»1779 (ex collectionibus a C. G. Andersson missis).

- In Öfvers. af K. Sv. Vet. Ak. Förl. 1888: „Ueber zwei Lamellibranchiaten aus den postglacialen Schichten Schonens.»

Ipse.

Nat. $12 / 11831$ in Vico Berga prope Kalmar, civ. acad. in Upsala 1853, doct. phil. in Lund 1862, collega scholæ in Ronneby 1863-1893, quotannis ab 1848 plagas nunc majores nune minores in provinciis plerisque Sueciæ et Daniæ perquisivi, me ad studium plantarum et animalium conferens, in ultimis annis socio et anxilio filiorum trium natu majorum, 1868 Norvegiam invisi, 1872 naturam Germaniæ, Austriæ et Italiæ, 1874 et 1881 eam Carinthiæ, Carniolie, Istriæ et Italiæ publice studere occasionem habui.

- Sveriges land- och sötvatten-mollusker. Lund 1865, pp. 142. Diss. acad.

- In Öfvers. af K. Sv. Vet. Ak. Förh. 1865, p. 537562: Malakologiska iakttagelser under en resa i Blekinge, Kalmar län och på Öland sommaren 1865 .

- In Nova acta reg. soc. sc. Upsal., 1871: Exposé critique des mollusques de terre et d'eau douce de la Suède et de de la Norvège, pp. 200 in 4:0.

- Fauna molluscorum terrestrium et fluviatilium Suecia, Norvegiæ et Danice. Sveriges, Norges och Danmarks Land- och Sötvatten-mollusker. Lund. I. 1871, II. 1873, pp. 651.

- In Öfvers. af K. Sv. Vet. Ak. Förh. 1881 p. 35-70: Malakologiska bidrag.

- Sveriges, Norges, Danmarks och Finlands Land- och Sötvatten-Mollusker. Exkursionsfauna. Stockholm 1884, pp. 76. 
- Fauna der in der Palä-arktischen Region lebenden Binnenconchylien, tomi 7, 1884-1890.

\section{Carl Gustaf Westerlund.}

Nat. ${ }^{23} / 61861$ in Ronneby, eiv, acad. in Lund 188', licent. phil. in Upsala 189.'

- In Nachrichtsblatt d. Malacol. Ges. 1889 p. 19-23: Wer ist der Verfasser der »Dissertatio academica Nova testaceorum Genera sistens», Lund: 1788?

- Bidrag till kännedomen om Ronnebytraktens Fauna och Flora, Stockholm 1890, mollusca p. 79-94.

\section{De molluscis norvegicis.}

Hans Ström.

Nat. 25/1 1726 in Borgsund Norvegix, depon. a Bergen 1743, sacellanns successoris patris 1750 , pastor eccl. par. Volden 1764, par. Eger 1775, nomine professoris honoratus 1780, doctor theologire 1790 ; den. in Eger $1 / 21797$.

- In scriptis suis "Beskrivelse over fogderiet Söndmöer» (62 ${ }^{\circ}$ l. bor.) 1762, »Beskrivelse over Egers Præstegjeld» 1784 et in Trondhjemske Selskabs Skrifter 1765 primus mentiones de molluscis norvegicis paucis fecit, scilicet Vitrina pellucida, anyelica (domestica), Helix hortensis, Hyal. (Hx.) hammonis n. sp. Balea perversa (Turbo perversus), Zua lubrica (Turbo muscorum), Pupa muscorum (Turbo cylindricus).

Clans. (Turbo) laminata?, bidentata n. sp., Nerita flaviatilis? (Nerita sp.) Margaritana (cujus naturam jam antea, 1753, Pontoppidan in sua Norges Naturlige Historie copiose tractavit).

\section{Joachim Friele.}

- Norske Land- og Ferskvands-Mollusker, som findes i Omegnen af Christiania og Bergen. Christiania 1853, pp. 65.

In hac prima molluscorum fauna norvegica descriptes sunt: Linax agrestis, Vitr. pellucida, Conulus (Hx.) fulvus, Vitrea (Hx.) crystallina, Hyalinia (Hx.) cellaria, nitidula (nitida), Arion rufus (forte a terris meridionalibus ad Bergen, ubi rarissima, introducta: Fr.), fuscus (subfuscus), Patula (Hx.) rotundata, ruderata (rot. var.), Helix pulchella, costata (pulch. var.), lapicida, hispida, strigella, fruticum, arbustorum, nemoralis, pomatia, Zua (Bulimus) lubrica, Pupa cylindracea, muscornm, pyg- 
mcea, Balea (Pupa) perversa, Claus. plicutula, öidentata (rugosa), dubia (rugosa pr. p.), Succinea putris (amphibia), Carych. (Anricula) minimum, Limncea stagnalis, auricularia, ovata, lagotis (ovata v. vulgaris), peregia, palustris v. fusca, glabra (elongata), truncatula, Physa fontinalis, hypnom, Plan. umbilicatus (marginatus), vortex, spirorbis, albus, contortus, nautileus, nitidus, Pahudina contecta (vivipara), Bythinia (Paludina) tentaculata. Talvata piscinalis, cristata, Neritina (Nerita) fluviatilis, Sphcerium (Cyclas) corneum, ovale (lacustre), calyculata, Pisidium obtusale, fontinale, Margarit. (Unio) margaritifera, Anodonta anatina.

\section{Edvard Karl de Martens.}

Nat. 18/4 1831 in Stuttgart, regni Württemberg, quo loco in gynanasia studia primo coluit, deinde a 1849-1852 artem medicam in Tübingen, 185̌3-185́ in München, doct. med. ib. 185̃ , in Berlin discipulus anatomiæ peritiss. Joh. Müller, præterea a juventute ardenter studiis malacologicis occupatus, extra patriam perquisivit partes Norvegiæ 1855 et Italiæ 1856, 1860-1863 zoologi loco in expeditione navis bellicæ Thetis ad Asiam orientalem particeps fuit, ab anno 1873 prof. zool. univ. in Berlin, scientiam malacologicam scriptis numerosis valde ampliavit.

- Ueber die Binnenmollusken des mittleren und südlichen Norwegens - in Malak. Blätter, 1856 p. 69-117.

- Ueber einige Landschnecken aus dem nördlichen Norwegen - in Sitzungsber. d. naturf. Ges. zu Berlin, 1881 p. 34 -39 (ex. collectione doctor. Aurel et Arthur Kraus, sub itinere 1880 inter $66^{\circ} 25$ et $67^{\circ} 37^{\prime}$ lat. bor. facta).

Novæ species sunt Limax marginatus $(\mathrm{E}$. de Mr. fere credit Limacem quem Ström e Söndmör descripsit hic esse), Hyalinia petronella et Clausilia biplicata (specimen unicum a prof. Sars s:r in ins. Manger inventum). Testam, quam s. n. Balece Sarsii Philippi (Zeitschr. f. Mal. 18.47) descripsit atque etiam ad Göteborg Sueciæ lectum dicitur (Pfr.) nihil aliud esse ac juvenis Claus. bidentatæ in Exposé critique (1870) et Fauna moll. Suec. etc. (1873) demonstravi.

\section{O. S. Jensen.}

- Indberetning om en i Sommeren 1870 foretagen Reise i Kristiania og Kristianssands Stift forat undersöge Land- og Ferskvands-Molluskerne tilligemed Iglerne. Christiania 1872, pp. 42 c. 1 tab. col.

De variatione limacum et distributione mollnscorum multum bene observavit, de cætero bene meritus faunæ norvegicæ multis noritiis ejusdem, scilicet: Limax maximus, r. leucogaster, v. fasciatus, r. ci- 
nereo-nebulosus, cinereus, levis, tenellus, Hyal. petronella, pura, (alliaria, antea a clariss. Steenstrup ad Arendal inventa), Arion ater v. medius nov., cinereo-nebulosus nov. (albus antea a Müller et Fabricius inventus), Helix hort. v. hybrida, arbust. v. depressa, hisp. v. depilata, aculeata v. sublevis, Zua lubr. V. minima, Pupa antivertigo, gravida n. sp. (Vert. pachygaster Jens. non Z. \& Rossm.), lilljeborgi ("Vert. ovata Say?" J.), alpestris, v. mitis tridentata, Claus. ventricosa, sejuncta, Succ. oblonga, arenaria, Plan. glaber, complanatus, Ancylus fluviatilis, lacustris, Pisid. milium, personatum et nitidum.

\section{Georg Ossian Sars.}

Nat. $20 / 4$ 1837, civ. acad. in Kristiania 1857, zool. prof. ib. 1874, præcipue studia animalium infer. marinorum coluit.

- Mollusca regionis arctica Norvegix, 1878.

Birgithe Esmarl, Natura curiosa virgo norvegica.

- In Nyt Magazin for Naturvid. 1879 p. 215-223: Bidrag til Kundskaben om Udbredelsen af Norges Land-og Ferskvandsmollusker i forskjellige Egne af Landet.

- Ibid. 1880 p. $77-110$ c. 1 tab.: Nyt Bidrag til Kundskaben om Norges Land- og Ferskvands-Mollusker.

- In Tromsö Museums Aarshefter 1882 p. 93-104: Land and Freshwater Mollusca in the arctic regions of Norway.

- In Journ. of Conch., London, V. p. 129-150: On the Land and Freshwater Mollusca of Norway.

- In Malak. Blätter N. F. 1883 p. 1-6: Die Pisidien des südlichen Norwegens.

- Ibid. 1886 p. 84-123: Die Land- und Süsswassermollusken des arktischen Norwegens.

Forte nec injuste nec inepte sit referre clar. Clessin Auctori de fauna norvegica meritissimæ familiam Sphæriidæ, me genus Planorbis, alios malacologos alia que colligit vel colligere curavit determinavisse. Novitix faum norvegicæ sunt: Vitrea contracta, Zonitoides norvegicus B. E., Helix arbust. v. picea (จ. fragilis B. E.), Pupa aretica, substriata, Succ. pfeiff. V. contorta, Limncea palustris $\nabla$. turricula, truncatula $\mathrm{V}$. microstoma, v. maximella. v. schneideri B. E., v. compressa B. E., Plan. albus v. cinctutus, borealis, V. Hexus, $\nabla$. angigyrus, strömi, concinnus, Sphcerium mamillanum, Pisidium globulare, obtusale v. esmarchianum, pulchellum, milium, subtruncatum, pallidum, pusillum, scholtzi. Anod. sulcata. 


\section{De molluscis danicis.}

\section{Eriti Pontoppidan.}

Nat. 24/8 1698 in Aarhuus Danire, prof. theol. in Kjöbenharn 1733, episcopus in Bergen Norvegix 1745, auctor deligens in historia et theologia, etiam scripsit „Försög til Norges naturlig:e historie" 1752-1754; den. in Kjöbenhavn 20/12 1764 .

-_ In Danske Atlas tom. I. 1763 p. 660 et 662 enumeravit 14 mollusea danica, ad Linnei Syst. Nat. X. relegens.

Hæc sunt: Arion (Limax) ater, Limax agrestis, maximus, Helix hortensis vel nemoralis (nemoralis P.), pomatia, Balea (Turbo) perversa, Plan. (Hx.) umbilicatus (planorbis), corneus (corneus et cornu arietis P.).. complanatus, Paludina ( $\mathrm{Hx}$.) vivipara?, Limncea $\left(\mathrm{Hx}_{\mathrm{x}}\right.$ ) stagnalis, fragitis, Anodonta (Mytilus) anatina.

\section{Otto Friederich Miiller.}

Nat. 2/5 1730 in Kjöbenhavm, parentibus germanicis, patri rectori scholx egeno, primo theologiam studuit, in annis 1763-1767 cum descipulo comite juveni Schulin itinera spatiosa fecit, postea custos tabularii in rario norvegico, deinde uxorem viduam mercatoris devitis ducente, se studiis historiæ naturalis omnino tradere posuit, denique consiliarius intimus danicus, den. 1784; et botanicus et zoologus meritissimus egregie in utraque scientia observarit et scripsit, præstat tamen præcipue opere fundamentali „Historia vermium», in quo studium malacologium terrarum borealium radicem habet.

- In opusculo »Efterretning om Svampe», 1763, nonnulla Limacina observavit, scilicet A. ater, albus, Lim. maximus, flavus?

- »Faunæ Fridrichsdalinæ novicia, que anni spatio in agro Fridrichsdalensi legi», in Flora Fridrichsdalensi 1767 p. 237.

- Historia vermium terrestrium et fluviatilium, vol. II. 1774 et ad finem ejus »Testaceorum agri Fridrichsdalensis, sen Danice, non marinorum indigenæ.»

In opusculo 1767 mollusca sequentia danica enumerata sunt: Arion ater, rufus, Limax agrestis. Alaus, Helix nemoralis, Succinea ( $\mathrm{Hx}$. putris, Limna (Hx.) stagnalis, auricularia, Planorbis (Hx.) umbilicaris (planorbis), complanatus, vortex, Ancylus (Patella) lacustris, Neritina (Nerita) fluviatilis, Spharium (Tellina) comeum, Anodonta (Mytilus) anatina.

In Historia Vermium desuper descripsit: Limax cinereus Lister (ciner. $\beta, \gamma$ ), tenellus (flavus pr. p.), pallidus Schrenk f. Mörch (agrestis 
$\boldsymbol{\varepsilon})$, lavis n. sp.; marginatus n. sp., Vitrina (Hx.) pellucida n. sp., Conulus (Hx.) fulvus n. sp. (sc. quoad descriptionem teste juvenis! Descriptio teste mature spectat certissime Hx. bidentem Chemn.!), Titrea (Hx.) crystallina n. sp., Zonitoides (Hx.) nitidus (et valiam albam hyalinam pellucidissimam striatam reperi; an hujus varietas? Mïll. $=$ Hy. nitidula f. Mörch), Hyal. cellaria n. sp. Arion (Limax) fuscus, cinctus, Patula (Hx.) rotundata n. sp., aculeata n. sp., pulchella n. sp.. costata n. sp., incarnata n. sp., fruticum n. sp., arbustomu, lapicida, nemoralis (non Linné quir primo H. hortensis Müll.) Buliminus ( $\mathrm{Hx}_{\text {. }}$ ) obscurus n. sp., Pupa (Hx.) muscorum (nec. Lin.). pusilla n. sp. (Vertigo n.; g'en.), Claus. (Hx.) laminata (bidens), (Cl. plicatula, bidentata et Balea perversa in sua Hel. perversa comprehendunturl), Carychium (n. gen.) minimum n. sp., Amphipeplea (Buccinum) glutinosum n. sp., Limnaca (Buccin.) peregra n. sp. (pr. p.) palustris n. sp., glabra n. sp., Physa (Planorbis) bulla n. sp., hypuorum (Plan. turrita), Plan. carinatus n. sp., spirorbis (vel leucostoma), contortus, imbricatus n., nitidus n. sp, Talvata (n. gen.) cristata n. sp., piscinalis n. sp., pusilla n. sp., Bythinia tentaculata (Nerita jaculator), Pisidium (Tellina) amnicum n. sp. Adhuc in opere landato errore plares ut species distinctas descripsit, quas clar. Mörch (in Syn. Moll. Dan.) sic interpretavit: „Plan. similis = corneus jun., „Plan. gelatinus» := Physa fontinalis jun., verosimiliter, „Helix sericea» = incarnata juv, »Nerita contorta» = Plan. umbilicaris monstr. »lateri umbilicali turrito, $\mathrm{N}$. sphxrica = Byth. tentac. jun., verosimiliter, Helix trochulus $=$ Bul. obscurus jun. Postea (in Prodr. Zool. Dan. 1776) addidit Anodontam cygneam et (in Vid. Selsk. Skr. 178S) Paludinam contectan (sub nom. vivipare, non Lin.).

\section{Casper Schade.}

Nat. $30 / 81754$ in ins. Fyen Daniæ, civ. acad. 1780, sacerdos 1883, pastor eccles. variis locis ab a. 1787, priepos. eccl. in Mors 1794; den. in Nyköbing 3/4 1828.

- Beskrivelse over Oen Morö, 1811; p. 23022 species enumeravit, nullam novam.

\section{Henrich Henrichson Beck.}

Nat. 1799 in Aalborg Danict, civ. acad. 1818, 1823 ab universitate danica nummo memoreali anreo propter tractationem systematicam papilionum Danice honoratus, doct. phil. Göttingensis, curator musei malacologici privati regis Christiani VIII, et adjunctus musei regalis in Kjöbenhavn; den. ib. 1863.

- Index Molluscorum prasentis avi Musei Principis Augustissimi Christiani Frederici 1837 et 1838. 
- In Amtlicher Bericht über die 24. Versammlung Deutscher Naturforscher und Aerzte in Kiel in September 1846, Kiel 1847, p. 122-124: Verzeichniss einer Sammlung von Landconchylien aus den Dänischen Staaten in Europa.

Faunam danicam his formis augmentavit in opere primo: Helix strigella, Pupa (Alæa) pusilla, antivertigo, angustior (Vertigo), Claus. biplicata, ventricosa, plicatula, sejuncta (pumila), bidentata (rugosa), Ceccilian. (Acicula) acicula, Succ. pfeifferi, oblonga (Bucc. peregr. Müll. specim. minora), Acme fusca, Limnoea intermedia Drp., succinea, balthica, vulgaris C. Pfi., ovata, lagotis, Plan. complanatus (fontanus), carinatus v. disciformis, leucostoma. Ancylus fluviatilis. De cietero, a clar. Mörch interpretatie, „Plan. deformis Lam. = Pl. umbilicatus specim. jun., „Plan. spirorbis» = Pl. umbilicatus specim. jun. minuta. - In opere secundo: Punctum (Hx.) pygmceum, Patula (Hx.) ruderata, Helix concinna (Pupa avenacea), Valvata macrostoma Stenb., Paludina contecta, Byth. leachi, Unio crassus, nec non e Islandia „Vitrina domestica Ström» et Limnera geisericola Bk. a cl. Faber lectæ.

\section{Johannes Japetus Smith Steenstrup.}

Nat. 8/3 1813 in vico Vang in Thy Danix, civ. acad. 1832, 1839-1840 Islandiam mandato publico et 1844 socio principis Frederici insulas Færö et Scotiam transivit, 1845-1885 prof., zool. in Kjöbenhavn, 1868 doctor honor. in Lund.

- In Kröyers Naturh. Tidskr. 1838-1839 de distributione molluscorum Daniæ multas observationes fecit et in faunam Hel. lamellatam et Cycl. elegantem introduxit.

- In opere suo laudato »Undersögelser over Hermaphroditismen» 1845 Pisid. obtusale, fontinale et amnicum enumerata sunt.

- In Amtlicher Bericht etc. 1846 dedit »Uebersicht der von ihm auf Island gefundenen Land- und Süsswasser-Mollusken.»

- In Malakol. Blätter N. F. 1. 1879 proposuit »Berichtigung rücksichtlich der von Herrn S. Clessin aufgestellten Limnæa Stenstrupi aus Island.»

Arthur Fredrick, Feddersen.

Nat. $16 / 21835$ in Kjöbenhavn, 1846-185̃3 alumnus gymnasii in Sorö, $180 ̄ 5$ examen polytecnicum absolvavit, $186 t$ adjunctus scholit in Viborg, præcipue studia ichtyologica coluit. 


\section{- In Viborg Cathedralskoles Inbydelseskrift til Examen} 1863: Til Blöddyrfaunaen omkring Viborg.

In hoc opusculo circa 80 species enumeravit, quarum 41 terrestres sunt. Hyal. alliaria, pura, Pupa substriata, Pisid. pusillum, pulchellum et nitidum sunt novitice faunce danicx ab eo detectæ.

Carl August Elberling.

Nat. $16 / 4183 \dot{x}$ in Slagelse Daniæe, civ. acad. 1851, magister in hist. nat. $1859,1861-1863$ adjunctus musei mineralogici, 1863 adj. bibliothecæ regalis in Kjöbenhavn.

- In Vidensk. Meddelels. fra Naturhist. Forening i Kjöbenhavn, 1862 p. $339-340,1864$ p. 279-280 et prxcipue 1870 p. 211-266: Undersögelser over nogle danske Kalktufdannelser. Ibidem 1876: Om] en Kalktufdannelse ved Veistrup Aa paa Fyen.

Nove famn sunt Helix mestris, Succ. putris v. trianfracta. pfeifferi v. intermedia Bean, Plan. draparnaudi.

In hac ordine referre volo quod noster S. Nilsson in Hist. moll. Suec. de molluscis Sueciæ fossilibus dixit. "Mollusca petrefacta terrestria et fluviatilia, ad species jam demortuas pertinentia, apud nos numquam inventa sunt. Quæ fossiles obveniunt species adhue vivunt. Tales sunt. ex. gr. Helix fruticum et $\mathrm{H}$. strigella in strato calcis tophosie (tuffkalk) ad Benestad Scaniæe, et plurime fluviatilium species in terra turfosa (torfjord) passim fiequentes obviæ. Omnià mollusca vere petrefacta, quæ in tellure Suecana occurrunt, marine sunt originis. Hoc omne etiamnunc post septuaginta aunos omnino rei et Suecire et Danie congruit, si tantummodo addere velis, nomnullas formas in ultimis annis Sueciæ inventas esse, quæ postglaciales quidem mortux sunt, sed maximam affinitatem enm viventibus habent. Tales sunt Helix costata v. cyclostoma, H. adela, Limnue prisca, L. palustris v. stenostoma, Valvata glacialis, Spharium subsolidum, Pisidium lindströmi. Alie subfossiles in terris turfosis regionum maxime meridionalium peninsulæ occurrunt, quæ nunc temporis non nisi vel præcipue in maxima boreali parte ejusdem vivunt, nt Pıtpa genesii, Planorbis borealis et Talvata alpestris.

\section{Otto Andreas Louson Mörch.}

Nat. ${ }^{17} / 51828$ in Lund Sueciæ, patri danico, custodi ad hortum academicum, ex matre suecana, tantum anniculus ad Daniam eum parentibus transmigravit, ubi postea conchyliorum studium ardore amplexus est, ita ut denique in scientia malacologica ernditissimus fuit, quod scriptis multis ostendit; den. pneumonia in Nizza $25 / 1888$. 
- In Naturh. Foren. Vidensk. Meddelelser 1863 p. 265 -367: Fortegnelse over de i Danmark forekommende Land- og Ferskvandsblöddyr. Opus separatim sub titulo Synopsis molluscorum terrestrium et fluviatilium Danix 1864 editum.

- Ibid. 1867 p. 67-111 \& 1868 p. 228--229: Fauna Nolluscorum Insularium Færöensium.

- Ibid. 1868 p. 185-227 : Faunula Molluscorum Islandia.

- In American Journ. of Conchology 1868 p. 41-45: On the Land and Freshwater Mollusca of Iceland.

Novitice faunæ danice sunt: Limax unicolor, heydeni, varieyatus (primum ad Christinehavn 1814 ab O. Fabricius inventus), Hyal. cellaria forma albina (Hy. margaritacea Mörch non Schm.), var. syleatica. Helix concinna, conspurcata, candidula, ericetorum, arbust. v. rudis, Pupa avenacea, costulata, edenlula, Claus. biplicata, plicata, dubia, parvula, succinea gracilis Alder», «S. albida Hartm.?», Limnea auvicul. v. canalis, v. ampla, ovata v. succinea, peregra v. paludinoides, v. atrata, Physa hypnorum v. perissiana, Plan. eloph. v. ammonoceras (corneus v. uordenskiöldi), glaber, Acme lineata?, Valvata nana (minuta), antiqua, Hyilvobia steini, Spherium ryckholti, rivicola, Pisid. feröense n. sp., henslowianum, milium. personatum, subtruncatum, Unio et Anodonta formæ multæe, Dieissena polymorpha.

\section{Christian M. Poulsen.}

Nat. ${ }^{14} / 31818$ in Aalborg Daniæ, 1838 examen pharmacia absolvavit, 1838-1842 adjunctus laboratorii acad. in Kiel, 1843 publice Germaniam, Italiam, Galliam etc. visit, doct. phil. Heidelbergensis, 1845 docens scholæ altæ populo aptre in Rödding; denatus 1886.

- In Naturh. Fören. Vidensk. Meddel. 1873: Bornholms Land- og Ferskvands-Blöddyr, pp. 15.

\section{Jonas Sigismund Collin.}

Nat. 8/4 $18\{0$ in Kjöbenhavn, civ. acad. 1859, magister zool. 1865, miles voluntarius in bello germanico-danico 1864. socio poëtre H. C. Andersen pluries Galliam, Italiam, Hispaniam, Atricam borealem etc. obiit et ubique studia malacologica coluit, ab a. 1870 munera publica ad culturas ostrearum Danice habet, præcipue mollusca marina studuit et scriptis illustravit. 


\section{De molluscis fennicis.}

Wilhelm Lilljeborg in K. Sv. Yet. Ak. Handl. 1850 multas observationes graves de molluscis in et ad lacum Ladoga tradidit.

\section{Alexunder Theodor de Middendorff.}

Nat. 6/8 1815 in Liflandia, doctor med., prof. zool. in Kiew, cura academix imp. in S:t Petersburg a. 1840 terras ad promontorium arcticum visitavit et $1812-1845$ Sibiriam borealem investigavit, de quo itinere opus magnum laudatum, ad historiam naturalem terrarum borealium maximi momenti, „Reise in den äussersten Norden und Osten Sibiriens (tomi \&, 1848-1875) edidit, $a b$ a. $185 \tilde{5}$ secretarius perpetuus acad. sc. in S:t Petersburg; denatus $28 / 1$ 1894. In opere citato etiam faunam malacologican Fenniæ multis observationibus illustravit.

Nils Adolf Erik Nordenskiöld \& Anders Edvin Nylander.

Nordenskiöld: nat. 18/11 1832 in Helsingfors Fennix, civ. acad. 1849 et doctor phil. ib. 1857, prof. mineralogire in museo regni in Stockholm 185̄8, annis 185̃8-1883 investigator illustris terrarum quam maxime arctic. pluries has terras investigavit, ibidem etiam hiemes transigit, precipue celebratus expeditionibus 1868 usque ad $81^{\circ} 39$ lat. bor., 1870 ad. Grönlandiam et 1878 -1879 per fretum Belningense circa Asiam et Europam, scientiis maximo lucro.

Nylander: nat. 1831 in Uleåborg Fennie, civ, acad. in Helsingfors 1848, cand. phys.-matem. 18533, doctor med. 1860, medicus urbicns 1861, medicus provincialis in Kuopio 1870, deuatus $23 / 51890$.

- Finlands Mollusker, Helsingfors 1856, pp. 110 c. 7 tab.

Mollusca fennica usque ad id tempus cognita in hac opere descripta sunt: Limax maximus (cinereus), agrestis, Vitrina pellucida, Hyalinia (Hx.) hammonis (pura), Conulus (Hx.) fulvus, Zonitoidles $(\mathrm{Hx}$.) nitilus Arion ater, mut. draparnaudi (var. ס), fuscus (subfuscus), Punctum (Hx.) pygmueum, Putula (Hx.) ruderata, Helix harpa (aculeata), costata, pulchella, lapicila, hispida, strigella, arbustorum, fruticum, schrenki, hortensis, Pupa muscormm, edentula, anticertigo, pygmea, substriata, pusilla, Balea percersa. ("lausilia laminata, plicatula, bidentata (nigricans), Zua lubrica, Succ. putris, pfeifferi, Carychium (Auricula) minimum, Amphipeplea glutinosa, Limneea stagnalis, v. borealis (var. $\beta$ ), v. livonica? (var. $\gamma$ ), auricularia, ovata, lagotis (vulgaris), v. balthica, peregra, palustris, v. fuscus, truncatula (minuta), Physa fontinalis, hypnorum, Plan. corneus, umbilicatus (marginatus), vortex, spirorbis, contortus, albus, crista v. imbricatus (nautileus), Ancylus 
Auviatilis, lacustris, Paludina contecta, vivipara, Bythinia (Paludina) tentaculata (impura), Valvata piscinalis, macrostoma (depressa), cristata, Neritina fuviatilis, Sphurium (Cyclas) corneum, calyculatum, Pisidium amicum (obliquum), obtusale, fontinale, Unio crassus, rostratus (pictorum), Margavitana (Unio), Anodonta sulcata (cellensis), anatina, 'ponderosa Pfr.:

\section{A. E. Nylander.}

- In Notiser ur Sällsk. pro Fauna et Flora Fennica Förh. (T. 4, a. 1859) p. 127-143: Bidrag till Finlands Malakologi. Opus jamdudum laudatum multis observationibus complevit.

\section{Joln Sahlberg.}

Nat. $6 / 61845$ in Helsingfor's Fenniæ, civ. acad. 1865, magistel phil. 1869, licentiatus 1879, docens acad. 1879 et prof. entomol. extraord. 1883.

- In Notiser ur Sällsk. pro Flora et Fauna Fennica Förh. 1875 p. 313̈-317: Anteckningar om några land- och sötvattensmolluskers utbredning inom Finlands naturhistoriska område.

Novæ sunt: Pupa alpestris et var. mitis, Planorbis carinatus.

\section{Manuscripta}

plus minusve completa et argumentosa Mollusca extramarina in variis terris scandinavicis tractantia amici sequentes suis temporibus benigne traduerunt:

C. Hartman $(\dagger)$, de molluscis in prov. Nerike inventis;

C. H. Johanson enumeravit mollusca in prov. Westmanland observata;

J. E. Zetterstedt $(\dagger)$ indicia dedit numerosa de distributione molluscorum in Suecia;

G. Eisen, de molluscis in Gotlandia et circa Stockholm observatis nec non enumeratio collectionis sux cum indiciis ubi formæ lectæ sunt;

$P$. de Laval $(\dagger)$, annotationes numerosa diligentesque de molluscis ab eo in plerisque partibus Sueciæ meridionalis et medice collectis;

C. G. Andersson, annotationes et observationes magni pretii de molluscis in prov. Dalarne ab eo per multos annos fac- 
tas nec non catalogum molluscorum in prov. Medelpad ab eo collectorum communicavit;

$E$. de Goës $(\dagger)$, index molluscorum in collectione sua magna asservatorum cum indiciis numerosis de habitatione formarum, nec non observationes multa et optimæ de familia Unionidx. Hanc collectionem studio submittere occasionem amplam postea habui;

E. Hemberg, de molluscis lapponicis et smolandicis;

Josua Lindahl, de molluscis scanicis;

J. Antarcrona $(\dagger)$, de molluscis in Blekingia orientali inventis;

O. Jensen, de molluscis norvegicis;

C. M. Poulsen $(\dagger)$, annotationes locuplitissimas ad faunam danicam dedit;

Steenbuch »Fortegnelse over Land-og Ferskvands-Blöddyr, samlede i Danmark, navnlig i Südwest-Sjælland»;

Jonas Collin, notitix numerose de fauna molluscorum Daniæ;

Herman Lynge, »Bidrag till den danske Molluskfauna»;

Ch. Rabot, franco-gallus, faunam malacologicam arcticam Fennixe circa Enare et Norvegix ad Pasvig (nec non illam peninsula Kola Russix) anno 1884 diligenter perscrutavit et collectionem totam cum annotationibus mihi benigne traduit.

\section{Collectio}

per decennia quattuor in itineribus et excursionibus facta et ab amicis multis liberaliter aucta tandem locupletissima fundamentum bonum cognitionis faunæ molluscorum harum terrarum præbet. In memoriam revocare volo scientiæ cultorum illorum, qui mihi collectiones nunc maximas nunc minores sæpissime pluries e regnis borealibus communicarunt et eo modo faunam illustrarunt. Sunt autem hi

A. d'Ailly, K. Ahlner, C. G. Andersson, J. Ankarcrona, P. T. Cleve, E. Colliander, P. de Laval, G. Eisen, A. T. Goës, Cl. Grill, C. Hartman, E. Hemberg, J. Henriksson, Fi. 
Holmström, L. P. Holmström, J. Hulting, R. Hägg, A. J. Johansson, C. H. Johanson, W. Lilljeljorg, J. Lindahl, G. Lindström, Cl. Linroth, V. Landberg, R. Landberg, A. W. Malm, G. Marklin, Erland Nordenskiöld, O. Nordstedt, A. de Post, C. D. E. Roth, O. Sandahl, F. Söderlund, Ph. Tarenius, H. D. J. Wallengren, J. A. Wallin, J. Fr. Ag. Westerlund, Hj. Westerlund, O. MI. Witt, J. E. Zetterstedt.

R. Collett, Laur. Esmark, H. Friele, O. S. Jensen, Ch. Rabot, J. Tidemand-Ruud.

A. Benzon, D. Bölling, Jon. Collin, Jon. Collin j:r, A. Feddersen, H. Lynge, O. Mörch, C. M. Poulsen, H. Sell, Steenbuch.

K. M. Levander, A. J. Mela, Fr. Tr. Mätilin.

Quos omnes cum maxima gratiarum actione nominare debeo. Nomina eorum in sequente brevitatis causa tantum literis initialibus citavi.

Occasionem etiam habui collectionem pretiosam professoris celeberrimi $P$. T. Cleve (nunc donatione cum Museo Zoologico Upsalise conjunctam) apud me studere.

Non omittam gratias maximas celeberrimis amicis meis gallicis reddere, H. Drouët in Dijon, procipue in Unionidis eruditissimo, qui benignitate sua consueta et sagacitate sua cognita plurimas formas novas in hoc opusculo allatas generis difficillimi Anodontæ ex speciminibus meis distinxit, et $N$. Locard in Lyon, infatigabili et meritissimo scrutatori molluscorum suie patrixe, qui formas boreales generum Bythinia et Dreissena, quorum monographias scripsit, examinavit.

Restat denique in memoriam revocare nomina eorum eruditorum, qui olim, scientiæ malacologicæ faventes, malacologos speciales nostrarum terrarum in curis suis adjuvarunt.

Pro Fauna Suecia S. Nilsson (1822) laudat »A. A. Retzium, Med. D:rem, qui in alpestribus Jemtlandixe et Norvegix iter pro Historia Naturali faciens, Mollusca quoque colligit, eademque mecum (S. N.) benigne communicavit: C. H. Boheman, Subcenturionem, qui in Smolandica et Westrogothica inquisivit et de hisce locis multa mihi exoptata impertivit: G. Marlilin, Hist. Nat. Studiosum, qui Uplandica specimina misit: A. Bruze- 
Tium \& B. F. Fries, Cand. Phil., qui Bahusiensia, Oelandica \& Scanensia, atque Landgren \& Sundevall, qui exemplaria in Scania collecta dederunt.»

Pro Fauna Norvegica B. Esmark nominavit $R$. Collett, $O$. Jensen, Schneider et Sophie Möller.

Pro Fauna Danica O. Mörch (1864) citavit Möller, Holst, H. P. C. Möller, J. Stecnstrup, Steenbuch, A. Feddersen, J. Collin, Liebmann, Ed. Erstev, N. Ch. Larsen, G. Budde-Land et N. C. Jörgensen.

Pro Fauna Fennica A. E. Nordenstiöld \& A. E. Nylander (1856) laudant $J$. J. Chydenius, H. J. Holnberg, F. W. Mätlin, A. de Nordmann et IV. Nylander. Hanc seriem nominum sequentibus augere possum (e Collectione molluscorum Fenniam habitantium in Museo Zoologico in Helsingfors asservata, quam totam ad studium apud me habui), scilicet $H$. Backman, A. Bodén, H. Lagermarck, K. M. Levander, W. Juslin, A. J. Ilela, O. Nordqvist, T. Renvall, $R$. Sievers et Aulis Westerlund.

Observes velim descriptiones omnes in hoc opusculo allatas ex speciminibus e locis septemtrionalibus citatis sumtas adeoque non raro iis ex speciminibus a terris meridionalibus sat dissimiles esse.

Asterisco (") signavi locum (sc. ubi illorum plures) originalem formarum novarum. 


\section{Cl. 1. Malacozoa Cephalophora.}

Mollusca capite libero, tentaculato, oculifero, testa mulla (interna) vel univalvi.

\section{Ordo 1. Inoperculata.}

M. hermaphrodita; testa nulla externa vel (plerisque) spiralis, inoperculata.

\section{Subordo 1. Geophila Fér.}

M. pulmonata, terrestria, tentaculis 4, retractilitus, superioritus 2 apice oculiferis.

\section{Sectio 1. Monotrema West.}

M. orificio org. genit. extremo communi.

\section{Fam. Vitrinidæ.}

Maxilla levis.

\section{Subfam. Limacina.}

Testa externa mulla, interna sub clypeo unguiformis. Corpus per totam longitudinem cum pede conjunctum, postice carinatum, orificio respirationis postico, clypeo concentrice striato.

\section{Gen. Limax Miill.}

Corpus sapissime fasciatum et carina abdominali atbida: clypeus corpore cetero multo brevior.

1. Eulimax Moq.Tand. Corpus magnum, firmum, clypeus postice sepius attenuatus, sudor non coloratus.

L. maximus Lin. (Syst. nat. $X$ 1758). Tentacula grosse granosa, corpus cinereo-nigrum, quadrifasciatum, rugis oblongis 
strictis, clypeus niger unicolor, crista pervalida, albida, solea pedis bicolorata. Long. 150-180, lat. 16-20 mm. (L. cinereo-niger Wolf.).

Hab. Suecia merid. (locus orig. ad Björnhofda Delandiæ) et media, saltem usque in Herjedalen (Funnäsdalen), passim vulgaris. - Norvegia meridionalis. - Dania tota, passim. - Fennia meridionalis, orientalis (Impilahti) et media, usque ad Vasa et Kaiana, vulgaris.

Mutationes principes:

niger, unicolor, carina nigra $\nabla$. albida, solea bicolorata. - Passim frequentior quam typus.

albus Paasch (Arch. f. Nat. Ges., 1843), unicolor. - Suec. in Bohuslän et Westergötland.

fasciatus corpus cinereum, fasciis 2 latis, nigris, undulosis, solea bicolorata. - Suec. in Blekinge (Ronneby). - Norr. ad Laurvik.

Aavescens, unicolor. - Suec. passim rarus.

flavidus, clypeus totus ater unicolor (marginibus flavidis nigromaculatis), postice late rotundatus, (antice emarginatus), corpus flavidum, superne fasciis 4 atris, lateribus parce fuscomaculatis, solea medio alba, lateribus brunnescenti albida. - Suec. in Södermanlaud (ad Dalbyö: E. N.).

cinereo-nebulosus Malm (Göteb. Vet. Samh. Handl., 1858), albicans, clypeo flavido, corpore postice maculato, solea tota albida. - Suec. in Bohuslän. - Norv. in Asker.

calosoma Eisen \& Stuxb. (Öfvers. K. Vet. Ak. Förh., 1868), clypeo atro, carina dorsi fusco-olivacea, lateribus pallidioribus, maculis oblongis olivaceis conspersis, solea medio albida, lateribus cinereis. - Suec. in Gotska Sandön prope ad Gotland.

leucogaster Mörch (Syn. Moll. Dan. 1864), brunneus v. nigricans, fasciatus (fasciæ interdum maculis albidis suppletæ), solea alba unicolor. - Suec. in Gotland (ad Ahr) et Blekinge (ad Ronneby rariss.). - Norv. ad Skien et Laurvik. - Dan. in Bornholm.

Var. cinereus Lister (Hist. anim. angl., 1678): tentacula minute granulosa, clypeus albocinereus, nigromaculatus, postice rotundate productus, corpus albocinereum, seriatim nigromaculatum v. quadrifasciatum, rugis angustis, solea albida, unicolor; long. 125-140 mm. - Snec. ad Stockholm et Ulriksdal (V. L.). - Norv, ad Laurvik et Arendal. - 
Dan. in Sjælland (multis locis vulgaris) et Jylland. - L. maximus Auct. pl.

Tax. unicolor Heyn. (Malak. Blätt., 1862): tentacula minute granulosa, clypeus non vel obsolete nigromaculatus, postice breve attenuatus, corpus cineremm, rugis angustis, flexuosis, solea alba, unicolor; long. 120-130 mm. - Suec. ad Romeby et ad Stockholm (Haga: J. WV.). - - Norv. ad Bergen. - Dan. in Sjælland (ad Frederiksdal).

Var. gyratus W. (Exk. Famn 1884): clypeus atrobrumens, concolor, postice omnino rotundatus, corpus atrum, rugis longis vermiformibus, valde flexuosis, solea medio alba, lateribus atra; long. $75 \mathrm{~mm}$. - Suec. in Blekinge (ad Bomanstorp pr: Romneby, rariss.).

Var, beryensis W. (Exk. Fauna, 188ł́); clypeus ater, maculis parvis albis nigrisque conspersus, postice brevissime rostratus, corpus atrum, fasciis 5́ pallidis, rugis adeo flexuosis, ut corpus forte nodulosum rideatur, solea alba unicolor; long. $100 \mathrm{~mm}$. - Norv. ad Bergen.

¿. Malacolimax Malm. Corpus parvum, mollissimum, vugis angustis acuminatis, clypeus postice rotundatus, sudor luteus, copiosus.

L. tenellus Nilss. (Hist. Moll. Su., 1822). Clypeus luteus, corpus luteo-virescens v. luteo-cinerascens, postice luteum, utrinque fascia fusca obsoleta, rugis in seriebus parallelis positis. Long. $30-60 \mathrm{~mm}$.

Hab. Suecia meridionalis in fagetis et aliis silvis humidis non rarus, rarior magis septemtrionem versus, sed usque in Medelpad $\left(62^{\circ}-63^{\circ}\right.$ l. b.) et Jemtland $\left(63^{\circ}-64^{\circ}\right.$ l. b.) inventus. - Norv. meridion. multis locis. -- Dania vulgaris in fagetis. - Fennio meridionalis (Hogland, Helsingfors, Abo) et media (Kuopio, A. J. Mela).

Mut. pheronotus Eis. \& Stuxb. (Öfvers. K. Vet. Ak. Förh., 18€8), clypeus luteo-cinereus, marginibus luteus, postice macula fusco-cinerea, fasciis duabus fuscis brevibus, corpus dorso fusco-cinereum, linea dorsali pallidiore et lateribus ccruleo-cinereis, solea medio cœrulescens, lateribus pallidis; long. $25 \mathrm{~mm}$. -- Suecia in Gotska Sandön prope ad Gotland.

3. Clepticolimax (Malm). Corpus magnum, firmum, rugis planis ellipticis v. oblongis, clypeus postice angulatus, sudor luteus. 
L. variegatus Drap. (TabI. Moll., 1801). Clypeus flavus, brunnescenti-marmoratus, corpus flavidum, brunneo-maculatum, carina albida. Long. $85--100 \mathrm{~mm}$.

Hab. Dania in cellis in Kjöbenhavn, ad Kristianshavn. (A. W. Malm semel numerosa specimina hujus speciei, e Gallia cum fructubus introducta, in cella quadam in Göteborg Suecire invenit, sed mox eadem ut noxia exstirpavit).

4. Lehmannia Heyn. Corpus majuscuhum, mollissimum, ut aquosum, postice pellucidum, triangulare, clypeus postice medio obtuse productus, sudor non coloratus.

L. marginatus Müll. (Verm. Hist. II, 1774). Cocrulescens v. cinereus, clypeus utrinque striga obscura, albomarginata, corpus dorso antice linea alba, postice carina acuta, alta, alba, utrinque fascia obscura marginata præditum. Long. $50-75 \mathrm{~mm}$.

Hab. Suec. merid. \& media late distributa et passim vulgaris, usque in Jemtland (ad Ljungan) et Herjedalen (ad Funnäsdalsberget), $62^{\circ}-63^{\circ}$ l. b. - Norv. merid. et media passim vulgaris. - Dania vulgaris. Færö, Islandia. - Fenni meridionalis (Abo: A. J. Mela).

Mutatio albomaculatus Mörch (Syn. Moll. Dan., 1861), „fascix pallii, extus linea alba intus maculis seriatim digestis, marginate, abdomen albomaculatum. - Dania ad Maglevandsfaldet in Mörens Klint.

Mut. pallens Less. ¿ Poll. (Mon. Lim. ital., 1882), supra pallide cinerascens, de cetero albidus. - Suecia ad Ronneby. Mut. mpicola Less. \& Poll. 1. c., supra ater, lateribus et subtus nec non interdum carina dorsali albidus. - Suecia in Jemtland (ad Kjolan: E. N.). - Islandia (A. F.).

\section{Gen. Agriolimax (Mörch) Simroth.}

Corpus numquam fasciatum, carina, si adest, concolor; clypens postice rotundatus, longitudine $1 / 3$ ad $1 / 2$ longitudinis totius cequans.

1. Chorolimax W. Corpus parvulum, molle, pallidum, unicolov v. fuscomaculatum, forte carinatum. postice longe acutcque productum, sudor lacteus.

A. agrestis L. (Syst. Nat. X, 1758, Limax). Clypeus 1/2 longit. 
totius æquans v. superans, colore valde variabilis, rugæe corporis oblongæe, planulatæ. Long. 30-60 mm.

Hab. Suecia tota (etiam ad Karesuando in Torne Lappmark ad $68^{\circ}-69^{\circ}$ l. b. inventus). - Norv., Dan. \& Fennia vulgaris.

Var. norvegicus W. (Exposé crit., 1871): uncini radulæ laterales denticulo postico muniti, testa interna crassa, conrexa, ad dextrum emarginata! (in typo testa tenuis, plana, marginibus hyalinis, uncini non denticulati). - Norv. in Ringerige (juxta viam in abietina $200 \mathrm{~m}$. s. m.*), Fiskum, Hole.

2 Hydrolimax Malm. Corpus parvum, gracile, firmum, unicolor, non vel breviter carinatum, cylindicum, postice brevissime acuminatum, sudor non coloratus.

A. heydeni Heyn. (Malak. Blätt., 1863). Inter A. agrestem et lavem medius, corpore breviter carinato, albido, Iutescente vel rufescente, tentaculis brunneis, sudore lacteo. Long. clypei $5 \mathrm{~mm}$., corporis $10 \mathrm{~mm}$.

Hab. Suecia ad Stockholm et Dania in Sjælland ad Kongekilden (O. M.).

A. lavis Müll. (Verm. Hist. II, 1874, Limax). Clypeus $1 / 2$ totius longitudinis aquans, profunde concentrice striatus, postice omnino rotundus, corpus nigrescente-brunneum, nitidissimum, rugis longis latisque, sudor aquosus. Long. 15$25 \mathrm{~mm}$.

Hab. Suecia meridion. et media sat vulgaris, saltem usque in Upland. - Norv. meridionalis. - Dania vulgaris. - Fennia meridionalis et media (K. M. L.).

Var. mucronatus W. (Exposé crit., 1871): luteo-brunneus, lateribus pallidis, solea alba, unicolor (in $\alpha$ pallide brunnea, medio albescens), clypeus postice processu conico; long. 10 mm. - Suec. ad Ronneby (Herrstorpsjön).

3. Arctolimax IV. Corpus parvum, firmum, dorso roturdum, convexum, postice sat subito angustatum. cauda perbrevi compresso et superne subcarinato, clypens postice late rotundatus, antice crassus ct forte dilatatus. Limacella oblonga, lateribus parallelis, antice truncata.

A. hyperboreus W. (Sibir. Moli., 1877). Supra ater, lateribus 
pallidis, subtus albidus, vel supra nigrescens, lateribus pallido brunneis. Long. 10, lat. max. $3 \mathrm{~mm}$. (Specim. in spiritu asservata).

Hab. Islandia (A. F.). Hæc species, qux per totam Sibiriam (l. o. ins. Sopotschnoj in Jenisej, $66^{\circ} 17^{\prime}$ 1. b. * $)$ et in Alaska America borealis occurrit, certissime in partibus borealibus peninsulæ scandinavicæ ac Fenniæ invenienda est.

\section{Subfam. Vitrinina.}

Testa externa adest. Corporis pars posterior a pede distincta, contorta et in testa inchusa, pallio magno ad dextrum appendicem lingureformem retrorsum extus testam emittente.

\section{Gen. Vitrina Drap.}

Testa depresso-globosa, subimperforata, temissima, hyalina, fragillima, sublevis; anfr. 3-4, ultimus maximus, apertura ampla.

V. pellucida Müll. (Verm. Hist. II, 1774, Helix). T. depressoglobosa, rotundata; anfr. 3, celeriter accrescentes, ultimus convexus, non dilatatus; apert. rotundata, margine columellari forte arcuato. Long. 4-5, lat. 3-4, alt. $3 \mathrm{~mm}$. Hab. Suecia tota usque in Lapponiam Lulensem (ad $68^{\circ}$ l. b.). - Norv. tota usqua ad $70^{\circ}$ 1. b. - Dania tota. - Fennia vulgaris uscue in Lapponiam extremam.

Var. dillwyni Jeffr. (Linn. Trans., 1830): t. globoso-depressa, anfr. ultimo perconvexo et spira altiore. - Passim.

Var. perforata W. (Fauna europ., 1876): t. minor et rotundatior, minute perforata, margine columellari multo minus arcuato, basi reflexo. - Suec. in Blekinge (ad Ronneby in Snäckbacken *).

Var. brunnensis Ulicny (Malak. Blätt., 188t): t. major, virescens, spira altiore, anfr. ultimo magis dilatato, sutura profundiore, striata; long. 7-8, lat. \& $\mathrm{mm}$. - Suec. ad Stockholm (Valdemarsudde in Djurgården: J. W.) et in Södermanland (Brännkyrka: E. N.).

V. angelicæ Beck (Ind. Moll., 1837). T. convexiusculo-depressa, subelliptica; anfr. 4, rapide accrescentes, ultimus dilatatus, 
depressus; apert. elliptico-rotundata, margine colum. forte arcuato. Long. $6^{1} / 2$, lat. 5 , alt. $3^{1 / 2} \mathrm{~mm}$.

Hab. Norv. in Nordlanden et Finmarken. Islandia. Fennia arctica (Ch. R.) et ad Solovetsk (Mus. Helsingf.).

V. holmiensis n. sp. T. imperforata, elongato-ovata, striatula, dense et distincte ad suturam albam marginatam descendentem, virescens; spira sat elata, longitudinis dimidiam vix æquans; anfractus 3, ultimus validus, forte aperturam versus dilatatus, supra convexo-declivis, subtus planulatus; apertura peroblicua, horizontali-elongato-triangularis, apice rotundata, margine superiore curvatulo-descendente, inferiore vix membranaceo-marginato. Long. 4, lat. $3^{1} / 3$, alt. $1 \frac{1}{2}$. mm.

Hab. Suecia ad Nackanäs prope Stockholm (Hj. W.)

\section{Subfam. Zonitina.}

Testa extema adest. Animal ut in Vitrinina, sed pallio in testa incluso, sine appendice.

\section{Gen. Conulus Titz.}

Testa subimperforata, globoso-turbinata, subtus fere plana, lcevigata; anfr. 5--6, angusti; apert. depresso-lunari:.

C. fulvus Müll. (Verm. Hist. II, 1774, Helix). T. turbinato-conica, fulva, striatula, subtus nitidissima, sublævis; anfr. ult. levissime subangulatus; apert. anguste lunaris, latitudine major. Lat. 3, alt. $2^{1} / 2 \mathrm{~mm}$.

Habitat totam Scandinaviam usque ad limites summos Lapponix et Finmarkix.

Forma alderi Gray (ap. Turton Brit. Sh., 1840), t. minor, fuscobrunnea, lævigata, polita. - Passim cum typo.

Var. mortoni Jeffr. (Linn. Trans,, 1830, Helix): t. depressa leviter conica, utrinque subæqualiter convexa, apice magno, anfr. regulares, ult. superne obsolete angulatus, infra convexus, apert. basi rotundata; lat. $2-21 / 2$, alt. $1 \frac{1}{2} \mathrm{~mm}$. Passim.

Var. praticola Reinh. (Sitz. ber. Ges. Berl., 1883): t. conica. latitudine et altitudine rqualis, brunneo-flavida, subtus spiraliter striata, anfr. rotundati, angulo obsoleto, apert. 
altior; lat. \& alt. $3-3 \frac{1}{2} \mathrm{~mm}$. - Suec. et Dania passim in pratis humidis.

C. fabricii (Beck nomen in Ind. Moll., 1837). T. conica, apice tenui; anfr. ultimus latior, subtus convexiusculus, medio impressus; apert. verticalis, late lunata, margine inferiore superne reflexiusculo. Lat. 4, alt. $3 \mathrm{~mm}$.

Hab. Islandia.

\section{Gen. Vitrea Titz.}

Testa anguste perforata ( $v$. imperforata), depressissima, orbicularis, vitrea, sublevis; anfr. 1-6, angusti; apert. verticalis, ungrestu, lunaris.

V. crystallina Müll. (Verm. Hist. II, 1774, Helix). Anfr. ultimus penultimo major, subtus tumidulus; apert. margine basali regulariter arcuato. Lat. $3-4$, alt. $1 \frac{1}{2}-2 \mathrm{~mm}$.

Hab. Suecia a prov. Skåne late distributa, sed sporadica et ubique rara, superiorem partem prov. Dalarne (fere $62^{\circ} \mathrm{l}$. b.) attendit. - Dania vulg. - Fennia?

Var. subterranea B. (Amén. malacol. I, 1856): t. depressior, umbilico latiore, sutura profundiore, anfr. convexis, apert. forte excisa, rotundata, peristomate albolabiato. - Suec. in Skåne, Blekinge et Westergötland inventa.

V. contracta W. (Fauna Moll. Su., 1873). Anfr. ultimus penultimo subrequalis, compressus; apert. margine basali subrecto. Lat. $2^{1 / 2}-3$, alt. $1 \mathrm{~mm}$.

Hab. Suecia in Skåne (ad Sjöbo: J. W.), Blekinge multis locis (l. o. ad Ronneby prope cataractam *), Westergötland (in Billingen), Öland (Borgholm). — Norv. ad Modum et Hole. Hrec species etiam in Germania, Transsilvania et Gallia inventa certe in Dania occurrit.

\section{Gen. Zonitoides Lehm.}

Testa aperte umbilicata, convexa, striata; anfr. lente accrescentes; ultimus paullo major; apert. lunato-rotundata.

Z. nitidus Müll. (Verm. Hist. II, 1774, Helix). T. latiuscula umbilicata, supra convexior, brunnea, nitida; anfr. 5-6, convexi, ult. supra convexior, Lat. 5-6, alt. $3 \mathrm{~mm}$. 
Hab. Suec. a Skåne ad Medelpad (saltem usque ad $63^{\circ}$ 1. b.). - Norv. meridionalis et media. - Dania vulgaris. Fennia usque in Lapponiam et ad Mare glaciale frequens.

Forma borealis Cless. (Malak. Blätt., 1878), t. depressa, vix convexa, sutura profundiore, canaliculata; lat. 6, alt. $21 / 2 \mathrm{~mm}$. Suec. ad Galtström in Medelpad (62 1. b.).

Var. parisiacus Mab. (Hist. mal. bass. Paris, 18 1 1): t. late umbilicata, convexa, anfi: convexi, ult. magnus, rotundatus; lat. $7-71 / 2$, alt. $31 / 2 \mathrm{~mm}$. - Suec. in Möllevången prope Nalmö.

Z. norvegicus B. Esm. (Nyt Mag., 1879). T. modice umbilicata, utrinque subærualiter convexa. virescens, anfr. $4^{1} / 2-5$, convexiusculi, ultimus subtus convexior. Lat. $7-7^{1 / 2} / 2$, alt. $3^{1 / 4}-3^{3} / 4 \mathrm{~mm}$.

Hab. Norvegia ad Unstad in Bamle, prope Langesund, sub plantis in collo aprico.

\section{Gen. Hyalinia Agass.}

Testa umbiicata, depressa; anfr. 4-7, regulares, ultimus maximus, depressus; apertura rotundata v. ovalis.

† T. minor (lat. $3-5 \mathrm{~mm}$.), concolor, anfi. $4\left(-4^{1} / 2\right)$.

H. pura Ald. (Cat. North. Moll., 1830, Helix). T. convexiuscula, subtilissime striatula et spiraliter lineata, cinereo-alba, absque nitore; anfr. ultimus major, rotundatus, vix depressus, non dilatatus, sutura impressa, non marginata; apert. subovata. Lat. $4-4^{1} / 2$, alt. $2-2^{1 / 2} \mathrm{~mm}$.

Hal. Suecia meridion. et media usque in prov. Dalarne passim. - Norv ad Modum. - Dania late distributa.

Var. lenticularis Held (Isis 1837, Helix): t. depressior, levigata, spiraliter lineata, anfir. ult. rotundior, non dilatatus, apert. rotundata. - Suec. in Blekinge. - Dania in Möen.

H. hammonis Ström (Trondhj. Selsk. Skr., 1765, Helix). T. depresso-convexiuscula, dense striata, corneo-brunnea (foeta castanea), pone aperturam fulvida, nitida; anfr. ultimus dilatatus, depressus, sutura linearis, marginata et crenulata; apert. rotundato-ovata, paullo depressa, interdum labiata. Lat. $3 \frac{1}{2}-4^{1} / 2$, alt. $2 \mathrm{~mm}$. - Hx. striatula Gray 
1821 (nomen), Hx. nitidosa Fér. 1822 (nomen), Hx. pura Malm 1851.

Hab. Suecia a prov. Skine in Lapponiam superiorem. Norvegiu tota usque in Finmarken. - Dania tota. - Fennia usque ad Mare glaciale.

Forma viridula Mke. (Syn., 1830), t. virescens, hyalina. - Præcipue in regionibus arcticis.

H. petronella Ch. ap. Pfr. (Monogr. Helic. IlI, 1853, Helix). T. convexa, dense costulato-striata, virescens (fœeta cinerea), pone apert. fulvido-albescens, hyalina, nitida; anfr. ult. rotundatus, non dilatatus; sutura ut præcedens; apert. rotundato-lunaris. Lat. $4^{1} / 2^{-}-5^{1} / 2$, alt. $2^{1 / 2--3} \mathrm{~mm}$.

Hab. In regionibus borealibus etiam summis vulgaris, in Suecia per regiones montanas usque ad Skåne, primo rarior deinde rarissima, descendit. - Norvegia tota. - Fennia tota.

H Teste major (lat. 6-1S mm.), subtus (circe umbiticum) albescens.

*) Spirce orbitu parca, anfi. (̌- 6 .

H. lucide Drap. ('Tabl. Moll., 1801). T. anguste umbilicata, circa umbilicum excavatula, spira convexa, subtectiformis; anfr. primi regulares, ultimus multo major, demum valde dilatatus; sutura impressa; apert. oblongo-elliptica, perobliqua, depressa, margine columellari forte curvato. Lat. 12-14, alt. $6 \mathrm{~mm}$. - Hx. draparnaldi Bk. 1837.

**) Spira orbitu magna.

H. ville Strob. Giorn. di Malacol., 1853). T. modice umbilicata, spira plana; anfr. lente accrescentes, ult. major, nou dilatatus; sutura canaliculata; apert. ovali-rotundata. parum obliqua, margine colum. parum arcuato. Lat. 14, alt. . $\mathrm{mm}$.

Hab. Hanc et precedentem speciem in Suecia prope ad Kalmar, certe cum saburra navium introductas, inveni.

H. cellaria Müll. (Verm. Hist. II, 1874, Helix). T. eylindricoumbilicata, porcellanea v. virenti-cornea, spira depressa; anfr. sat regulares, ult. major, rotundato-depressus, non dilatatus; sutura impressa, marginata; apert. ovali-lunaris. Lat. 12 , alt. $4 \mathrm{~mm}$.

Hab. Suecic passim a prov. Skåne ad Stockholm (Haga et Sabbatsberg: J. W.) et Upsala (P. T. G.). - Norv. ad Bre- 
vik, Porsgrund, Langelund et Bergen. - Dania multis locis et in insulis et in Jylland. - Fennia meridionalis in paucis locis (ut Åland, Kastelholm, Karelen).

Forma albina, albido-cœrulescens, subopaca. - Rara.

Var. silvatica Mörch (Syn. Moll. Dan., 1864): t. sat convexa. satura impressula, dense striata, apert. intus, pracipue margine columellari, late tenueque labiata; lat. 11-13, alt. 5-6 mm. - - Suec. ad Kalmar et Carlskrona. - Dania in silva ad Fredriksdal * in Sjelland, in Möen et ad Veile in Jylland.

H. angulata nov. sp. T. mediocriter et perspective umbilicata (umbilicus ab apice omnes anfractus regulariter angusteque accrescentes præbens, ad aperturam ne minime dilatatus), depressa, convexiuscula, apice paullo prominula, sub epidermide tenui fulvida albescens, nitida, dense tenuissime striatula; anfr. 5, convexi, regulariter accrescentes, ultimus penultimo circa $1 / 4$ latior, ultimus lentissime acerescens, supra convexiusculus, infra convexior, at planatus, supra medium obtuse angulatus, angulo fere ad aperturam conspicuus; sutura impressa, marginata, antice recta; apertura horisontalis, late lunari-ovata, extus rotundata, margine columellari arcuatulo. Diam. $7^{1} / 2$. alt. $3^{1 / 2} \mathrm{~mm}$.

Hab. Dania in Kjöbenhavn (in horto botanico vetero: H. L.).

H. nitidula (Drap.??) Rossm. (Iconogr. f. 24, 1835?, f. 256, 1838!) T. aperte umbilicata, supra rufa v. rufescens, subtus albescens, firma, spira convexa, supra depressa; anfr. sublente regulariter accrescentes, ultimus rotundatus, utrinque convexus, ne minime dilatatus, sutura sat impressa; apert. rotundata, margine basali arcuato; umbilicus lente regulariter dilatatus. Lat. $7-9$, alt. $3^{1 / 2}-4^{1} / 2 \mathrm{~mm}$.

Hað. Suecia in Skåne, Blekinge, Småland, Öland et Gotland, passim, rarissime. - Norv ad Kristiania et Bergen. Dania multis locis et in insulis et in Jylland.

H. alliaria Mill. (Ann. Phil. n. s., 1822, Helix). T. subaperte umbilicata, rufocornea, nitidissima, tenuis, tenuissime stria- 
tula, spira convexiuscula; anfr. regulares, ultimus rotmndatus; sutura plana, tenue marginata; apert. rotundata. Lat. $6-7$, alt. $3-3^{1} \frac{2}{2} \mathrm{~mm}$. Animal nigrum, allio oleraceum.

Hal. Suecia passim rarissime a prov. Skîne ad Upsala (in Hortu botanico: P. T. C.). - Norv. ad Arendal, Modum, Hole, Norderhoug, Herstad etc. inventa dicitur, si recte determinata. - Dania multis locis in insulis et peninsula; Islandia.

Mut. viridula Jeffr. - Rarior.

Var. anceps W. (Fauna Sueciæ, 1871): t. anguste umbilicata, depressior, rirescens, anfr. sat celeriter accrescentes, ult. paullo compressus, apert. diagonalis, lunato-ovalis, margine colum. levissime arcuato; lat. 6 , alt. :3 mm. - Suec. in urbe Lund (in loco Paradislyckan dicto).

H. sylvicola Paul. ap. W. (Fauna der Binnenconch. 1, 1886), forma scanica IT. l. c. T. excavato-umbilicata, late convexa, olivaceo-brunnea; anfir. regulares, ultimus penultimo duplo latior, subtus planatus; sutura impressula, marginata; apert. oblongo-ovata, margine basali ab insertione substricte arcuatulo; umbil. lentissime ampliatus. Lat. 10, alt. $5 \mathrm{~mm}$.

Hab. Suecia ad Belteberga (in silva prope fontem) in Skåne.

H. upsaliensis W. (Verh. zool.-bot. Ver. Wien, 1892). T. sat anguste umbilicata, subdiscoidea, olivaceo-micans, rufobrunnea, pracipue ad suturam impressam et marginatam dense striata; anfr. primi lente, cieteri forte accrescentes, ultimus extus rotundatus, subtus planatus et in centro late concavus; apert. rotundato-ovata. Lat. 12 , alt. $4^{1} / 2 \mathrm{~mm}$.

$H a b$. Suecia in Upsala (in Horto botanico, unde numerosa specimina benigne misit Prof. Gleve).

H. nitens Mich. (Compl. moll. 1831, Helix). T. dilatate ac sat late umbilicata, depressa, brunneo-lutescens v. rufescens; anfr. primi lente accrescentes, ultimus depressus, antice valde et subito dilatatus; apert. ovato-elliptica margine basali ab insertione subverticali arcuato; umbilicus demum celeriter ampliatus. Lat. 9, alt. $4 \mathrm{~mm}$. 
Hab. Suecia in Skåne (Lund, Belteberga, Smedstorp, Ringsjön etc.), Blekinge, (Ronneby, Carlskrona, Valjö), Småland (Nydala), Södermanland (Ålberga), Dalsland (Heden et Norra Bäckebo: J. H.), Westergötland (Borås, Kinnekulle, Hunneberg, Mösseberg), Gotland (Klintehamn: E. N.), Stockholm (Haga: J. WV.). - Norvegiu ad Langesund. - Dania in plerisque silvis fagineis in Sjalland et Bornholm.

Forma helmii Gilb. ab Gray (in Turt. Manual, 1840), t. unicolor, lactescens. - Dania ad Kongekilden in Sjælland et ad Kanegaard in Bornholm.

Var. olearis W. (Nachr. bl. Mal. Ges., 1883): t. aperte umbilicata, valde oleo micans, olivaceo-brunnea, subtus vix pallidior, dense tenuissimeque spiraliter lineata, anfr. sat regulares, ultim. antice parnm dilatatus, sutura non marginata, crenulata, apert. margine basali leviter arcuato; lat. 9, alt. $5-51 / 2 \mathrm{~mm}$. - Suec. Öland ad Borgholm et in Blekinge (Romneby in silva fag. pr. Pehrsborg*). - Dania ad Frederiksdal prope Kjöbenhavn.

Var. lundensis W. (Fauna d. Binnenconch. 1, 1886): t. firma, vix nitidula, utrinque dense regulariter striatula, rufescenticornea, subtus albida, sutura non marginata, apert. elongato-ovata; lat. 10 , alt. $5 \mathrm{~mm}$. - Snecia in urbe Lund.

\section{Fam. Helicidae.}

Maxilla verticaliter costata, margine crenato.

\section{Subfam. Arionina.}

Testa externa mulla (interna sub clypeo granulis catcareis suppleta). Corpns animatis per totam longitudinem cum pede conjunctum, orificium respirat. antemedianum. clypers granulosus, fovea candalis mucipara.

\section{Gen. Arion Fér.}

† Animal nagnum, non fasciatum, sudore non colorato, limbo pedis lineis transversis fuscis.

A. ater L. (Syst. Nat. X, 1758, Limax pr. p.) A. corpore toto superne grosse squamoso, squamis elongato-lanceolatis apicibus alternantibus, clypeo postice subtruncato, supra omnino atrum. Long. 100-120 mm. 
Hab. Suecia merid. et media, saltem usque in Westmanland et Upland vulgaris, regionibus campestribus in Skåne, Öland etc. exceptis, ubi rarus (ad Borgholm vulgaris et solus). Norv. merid, et media. -- Dania vulgaris. - Fennia? - Fide clar. Pollonera hee species et sequens solummodo in his terris occurrunt. - In Ifö Scanix specimina usque ad $150 \mathrm{~mm}$. longa vidi.

\section{Mutatlones:}

mellius Jensen (Indberetning, 1872), supra ater, clypeo antice et lateribus pallide brunneo, abdomine lateribus albo, capite cum tentaculis cinereo-nigrescente, limbo pedis aurantiaco. - Norv, ad Bergen.

cinereo-nebulosus Jensen 1. c., clypeo antice, lateribus corporis et solea obscure cinereo-punctatis, dorso albescente, limbo luteo. - Norv, ad Nis.

albus L. (Syst. Nat. XII, 1767, Limax), totus albus v. tentaculis et limbo interdum coloratis, hoc sæpe lineis transversis subnigris; sæpe flavidus. - Suecia in Skine, Blekinge, Halland, Bohuslän, Westergötland, Nerike, Westmanland et ad Stockholm inventus, in utraque provincia sæpissime sporadicus et rarus, locis tantum nonnullis frequens. Norv. meridionalis raro observatus. Dania in Sjelland, Bornholm et Jylland.

A. empiricorum Fér. (Hist. Moll., 1819). A. corpore toto superne rugoso, rugis per totum corpus porrectis subparallelis, densissime forteque flexuosis, clypeo postice subrotundato, versicolor, sed apud nos sæpissime atrum. Long. 80$120 \mathrm{~mm}$. - L. ater Lin. pr. p.

Hab. Sueciu, Norvegia et Dania passim, ubique rarior quam A. ater (in Blekinge ad Ronneby vulgaris et solus).

\section{Mutationes:}

miilleri Moq.-Tand. (Hist. Moll. II, 185̃), ater, carina dorsali pallide virente. - Dania sin nemoribus» (Müll.).

marginatus Moq.-Tand. 1. c., niger, limbo lutescente, aurantiaco vel coccineo, subtus albus. - Norv. ad Laurvig. - Dania » in horto Frederiksdalensi rarior» (Müll.).

it Animal mediocre, utrinque srepissime fascia fusca ornatum.

*) Dorsum abdominis rotundatum.

a. - Limbus pedis transversim plus minusve nigrolineatus; fascia lateralis clypei et abdo- 
minis continua, si adest; sudor luteus $\iota$. autrantiacus.

A. rufus L. (Syst. Nat. X, 1758, Fauna Su. ed. 2., 1761, excl. ș̣n., Limax). Supra rufus v. rufescens, dorso, medio clypei et abdominis fusco, utrinque non $\mathrm{r}$. obsoletissime et diffuse fasciatus; dorsi squamie angustæ, obtusæ, crenatæ v. rugulose, sulcis profundis disjunctr; clypeus dense granulosus; tentacula nigra; solea integra. Long. 40-70 mm. - L. ater var. $\delta$ Müll. 1874! L. rufus Nilss. 1882 (excl. synon.), A. rufus TI. 1873, Prolepis fuscus Malm 1868 pr. p. (t. 2 f. 3).

Hab. Suecia meridionalis et media rarior, at late distributa. - In Norv. ad Bergen et Hardanger. - Danic in horto ad Fredriksberg. - Fennic in Aland, in par. Muurla, Ingo et ad Kirjavalaks.

Obs. Certissime errore L. rufus L. ad A. atrem L. ducitur, enim mutatio hujus rufa Mich., in terris meridionalibus Europæ multo quam atra vulgatior r. sola, in Suecia numquam ab ullo reperta est, quod satis superque sit!

Tar. laris mh.: supra brunnescenti flaridus, efasciatus, lateribus infia medium flavidus, clypeus obsoletissime granulosus $v$. potius lævis, postice truncato late rotundatus. dorsi squamæ anguste lanceolatæ, utrinque acuminatæ, obsoletæ, sulcis levissimis disjunctæ, levibus pedis transverse fuscostriatus; animal in spiritu $30 \mathrm{~mm}$. longum. - Snecia in Jemtland (ad Kirokom: E. N.).

Var. fuscus Müll. 1. c.: ssupra rufescens. dorso clypei \& abdominis macula longitudinalis fusca, utrinque linea nigricans clypei sinuata, subtus albus, tentacula nigra; long. \& lin." (15 mm.) Müill. - Suecia merid. et media rulgaris, septemtrionalem versus usque ad Herjedalen (Tunnäsdalen et Midtådalen), Jemtland (Brasta), Öfrer-Luleå Sarestö, et Luleå Lappmark (Qrickjock). - Norr. tota usque in Ostfinmarken ad Mare glaciale. - Dania rulgaris. - Fennia tota. - Animal raro quadrifasciatum.

Var. cinctus Müll. 1. c.: ssuccini coloris supra absque omni macula, subtus totus albus, clypeus et abdomen dorso striga cinerea cingitur; long. 2 unc.s - Mïll. -- Hab. cum rar fusco. - L. fasciatus var. $\varepsilon, \zeta, \eta$ Nilss.

Var. Urunneus Lehm. (Schn, u. Musch. Stettin, 1873): semiteres, ochraceus v. badius, supra fuscus, lateribus pallidior, 
fasciis lateralibus nullis; (clypeus maculis fuscis nebulosis obscuratus, fasciis carens, dorsum fuscatum, interdum confuse zonatum»: Pollon.!); long. 45 , lat. $7 \mathrm{~mm}$. - Norvegia (H. Simroth).

A. limacopus IV. (Exposé crit., 1871). Supra rufobrunneus, lateribus sensim pallidioribus; clypeus irregulariter rugulosus; tentacula fusca; solea sulcis 2 longitudinaliter tripartita, partibus lateralibus lineis transversis, parte mediana subelevata. Long. $40-50 \mathrm{~mm}$.

Hab. Suecia in Blekinge (ad amnem prope cataractam", Ronneby) et Skåne (ad Stehag).

b. - Limbus pedis non v. obsolete fuscolineatus, fascice laterales nulle, sudor flavus $r$. luteus.

A. flavus Nilss. (Hist. Moll. Su., 1822). Sat gracilis, flavus r. dorso leviter cinerascens, capite et tentaculis nigris, limbo pedis flavescente, unicolore, solea albida. Long. 30-40 mm. Hab. Suecia in Skåne et Blekinge in plerisque silvis fagineis, sed sat rarus, in Smăland (fide Nilsson). - Nort. in Ringerige. - Dania prope Kjöbenhavn (in Dyrehaven prope Bellevue).

A. citrinus II. (Exposé crit., 1871). Citrinus, supra dorso vix visibile obscurior, cervice et tentaculis nigricantibus, clypeus elongatus, dense granulosus, corpus squamis oblongis, limbus pedis pallidus vix transverse nigrostriatus, solea alba. Long. $45-50 \mathrm{~mm}$. In statu contracto corpus priecipue animalis junioris aculeis obtectum videtur. Species maxime uncinis radulae et systemate reproductivo, a clar. Babor in Prag descripto, insignis.

Hab. Suecia in fagineis plerisque prov. Skåne et Blekinge (locus orig. ad Öfvedskloster * in Skåne). - Norv. in Ringerige (Herfjellet). - Dania? (verosimiliter in fagineis).

c. - Limbus pedis numquam transierse nigrolineatus: fascia lateralis clypei postice ascendens, ab illa corporis sejuncta: sulor pallide citrinus.

A. nilssoni Pollon. (Atti Accad. sc. Torino, 1887). A. hortensi similis, sed griseoflavescens, supra medio fuscatum, rugis angustis, dorso crassius verrucoso, clypeo brevissimo. Long. 
$\max .55 \mathrm{~mm} . \quad-$ Limax fasciatus Nilss. $\alpha, \beta, \gamma$ pr. p.; Prolepis hortensis Malm (Limacina t. 2 f. 5). Præcipue notis anatomicis singularibus distincta.

Hab. Vide sub A. bourguignati.

? A. hortensis Fér. (Hist. Moll., 1819). Nigrescens, fasciis lateralibus superne lineis longitudinalibus flarescentibus terminatis, inferne sensim evanescentibus, limbo flaro v. aurantiaco, solea pallide cinerea. Long. $30-35 \mathrm{~mm}$.

Hab. Vide sub A. bourguignati.

***) Dorsum abdominis linea longitudinali merlia elevata alba: sudor liquosus $i$. erystallinus.

A. bourguignati Mab. (Arch. malacol. I, 1868). Cinereus, dorso fuscato, fasciis lateralibus fuscis infra prorsus terminatis, limbo pedis pallido, vix conspicue atrolineato, solea albida. Long. 30-40 mm. - Limax fasciatus Nilss. pr. p.

Hab. Arion nilssoni, hortensis et hæe species apud nos, ut etiam in plerisque aliis terris, sub nomine hortensis confusi sunt, qua causa distributio eorum incerta est. Scimus formam, quam omnes auctores scandinavici A. hortensem nominaverunt, in Suecia a Skåne saltem usque ad $64^{\circ}$ lat. bor., in Norvegia meridionali multis locis et in Dania saltem in Sjæelland et Jylland sat frequenter occurrere, sed suspicor ut A. bourguignati et nilssoni sub hoc nomine sæpissime (si non semper) sumti sint. Dies docebit an ita sit.

Observationes nomnulla systematica ad Limacina et Arionina borealia:

Limax flaw L. (exclus. synon. Listeri!) = L. agrestis mutatio argillaceo-flava, dense brunneo-maculata, apud nos omnium Limacidum ubique vulgatissima. Cfr. W. in Fauna Suec. p. 69!

Limax flaves Mïll. pr. p., vclypeo brevi tumido rugis concentricis instructo») = Limax tenellus Nilss.

Limax flavus Müll. altera pars (»long. $11 / 2$ unc., supra flarus absque omni macula, subtus albus, tentacula lineaque inter hec et clypeum nigra, clypeus imprimis flavissimus absque rugis concentricis» - in nmbrosis Dania et Norvegia) $=$ Arion cinctus Müll. var. sensit, et verosimiliter jure, O. Mörch.

Limax flavus ("L.») Mörch et Eulimax favus (» L.») Malm = L. variegatus Drap., species in Snecia numquam reperta! 
Limax tenellus Müll. = Arion ater, pullus!

Limax succineus Miill. = Limax agrestis, mutatio, (supra subrufus, subtus albus») et forte etiam L. tenellus Nilss.

\section{Subfam. Helicina.}

Testa externa (ut in omnibus molluscis sequentibus) adest, forma depressa, globosa al conoidea; apertura sapissime transversalis, latitudine major. Corpus animalis parte posteriore a pede distincto, contorto et in testa incluso.

\section{Gen. Punctum Morse.}

Testa minutissima, late umbiticata, planata, apertura late lunari-rotundata, peristomate simplici, recto.

P. pygmæum Drap. (Hist. Moll., 1805, Helix). T. cornea, ubique striatula; anfr. 4, convexi, angusti, regulares. Lat. $1 \frac{1}{2}-2$, alt. $3 / 4-1 \mathrm{~mm}$.

Hab. Scandinavia tota: exiguitate invita in Dania tota, in Suecia usque ad Qvickjock in Lule Lappmark, in Norvegia usque in Vardö Finmarkixe (ad $70^{\circ} 20^{\prime}$ l. b.) inventum et in Fennia usque ad Mare glaciale

\section{Gen. Pałula Held.}

Testa late perspective umbilicata, costulata v. costuta, apertura rotundata, peristomate simplici, acuto.

P. rotundata Müll. (Verm. Hist. II, 1774, Helix). T. typice planata, costulata, brunnea, ferrugineo-maculata; anfr. $6-7$, planulati, ult. compressus, superne angulatus, subtus convexior; apert, rotundato-lunaris. Lat. 5-6, alt. $2 \mathrm{~mm}$.

Hab. Suecia merid. vulgaris, media rarior usque ad Stockholm (ad Haga, ad Stafsund in Eckerö, etc.). - Norv. meridionalis. - Dania tota.

Var. globose Fried. (Malak. Blätt., 1864): t. minor, convexa, spira elata. - Suec. in Öland (Borgholm).

Var. turtoni Flem. (Brit. Anim., 1823): t. depressissima, spira fere plana. - Suec. ad Kalmar (in loco „Balkplanens extra urbem frequens). 
Obs. P. rotundata Miill. certissime eadem est ac Helix gothica Lin. in Syst. Nat. X, 1758 (st. carinata, utrinque convexa, cornea. fasciis subferrugineis. - Habitat in Suecix nemoribus»). Pro y fasciis» tantmmmodo est legendum: fasciis transversis v. maculis. In Fauna Suecica ed. 2. 1761 hrec species oblita est. Plures auctores mirabiliter. cum Helice arbustorum L. conjungunt.

P. ruderata Stud. (Kurz. Verz., 1820, Helix). T. convexa, dense costulata, lutescenti-cornea, concolor; anfr. 4-5, ult. rotundatus, medio obsolete angulatus; apert. subovato-lunaris. Lat. $7-8$, alt. $3-4 \mathrm{~mm}$.

Hab. Suecia a Lule Lappmark usque in Smolandiam montosam frequens, deinde in Blekinge, Skåne, Öland et Gotland raro v. rarissime occurrit. In mare balthico subfossilis in terra turfosa, submarina extra urbem Ystad, Scanixe quam maxime meridionalis, ubi nullum vestigium $P$. rotundatæ, frequenter inventa. - Norv. tota et pracipue in regionibus borealibus frequens. - Dania perraro in Sjielland (ad Fredriksdal) et Bornholm. - Fennia tota usque ad Mare glaciale frequens.

?P. striatella Antony. T. pricedenti similis, sed depressior nec non distantius et fortius costata.

Hal. Hanc in America boreali indigenam speciem e »Lappland» communicavit Instit. zool. »Linnae» in Berlin.

P. rupestris (Stud.) Drap. (Tabl. Moll., 1801, Helix). T. minuta, aperte umbilicata, turbinato-depressa, dense striata, corneobrunnea; anfr. 5, cylindracei, angusti, ultimus subrotundatus; apert. rotundata. Lat. $2-3$, alt. $1 \frac{1}{2}-2 \mathrm{~mm}$.

Hab. Dania in peninsula Jylland ad Neder Knapperup (subfossilis in stratis calcis tophosa: E. Elberling).

\section{Gen. Helix Lin.}

Testa forma pervariabilis (depressa, globosa, conoidea), apertura transversa, lunaris $v$. rotundata, peristomate simplici, labiata $v$. dentata.

Subgen. 1. Vallonia Risso. T. aperte umbiticata, depressa; anfr. $3^{1 / 2}-4$, regulares; apert. subcircularis, peristomate reflexo, labiato. 
H. costata Müll. (Verm. Hist. II, 1774). T. planata, cinereoalbida, costata; anfr. ult. non dilatatus, antice deflexus; apert. perobliqua, peristomati late expanso, labiato, marginibus subcontiguis. Lat. 3, alt. $1 \frac{1}{2} \mathrm{~mm}$.

Hab. Suecia merid. et media usque ad Medelpad (63 I. b.) - Norv. usque ad $70^{\circ}$ lat. bor. - Dania vulgaris. Fennia verosimiliter tota.

Forma cyclostoma W. (Öfv. K. Vet. Ak. Förh., 1881), apertura callo alto contigua, peristomate subsoluto. - Suecia in terra turfosa submarina ad Istad prov. Skåne.

H. pulchella Mïll. (Verm. Hist. II, 1774). T. planata, alba, subtiliter striatula; anfr. ult. paullo dilatatus, non deflexus; apert. vix obliqua, peristomate late expanso, labiato, marginibus disjunctis. Lat. $2^{1} / 2$, alt. $1^{1 / 3} \mathrm{~mm}$.

$\mathrm{Hab}$. Ut procedens, sed ubique rarior.

H. adela II. (Öfvers. K. Vet. Ak. Förh., 1881). T. convexa, alba, striata; anfr. ult. vix dilatatus, paullo descendens: apert. peristomate angustissime limbato, vix labiato, marginibus disjunctis. Lat. $3-3^{1 / 3}$, alt. $1^{1 / 2}-2^{1 / 4} \mathrm{~m}$. m.

Hal. Suecia in palude bituminoso submarino baltico ad Ystad in Skåne frequens subfossilis. Vivit in alpibus bavaricis.

Subgen. 2. Acanthinula Beck. T. perforata, globosa v. turlinata, membranaceo-lamellata; apert. lunaris v. rotundata, preristomate sepines simplici.

H. Iamellata Jeffr. (Linn. Trans., 1831). T. conoideo-globosa, apice late rotundata, flavido-cornea, membranaceo-costulata; anfr. $6 \frac{1}{2}$, angusti, rotundati, suberquales; apert. depressa, lunaris, non labiata. Lat. $2 \frac{1}{3}$, alt. $2 \mathrm{~mm}$.

Hab. Suecic in Skåne (Westra Wram, Askeröd, Båstad, Askeröd, Tågra, Hallandsås et copiosiss. in Skäralid), Östergötland (Omberg ad Borghamn) et semel multis ante annis in Blekinge ad Ronneby inventa. - Dania in plerisque silvis plus minus frequens.

H. aculeata Müll. (Verm. Hist. II, 1774). T. globoso-turbinata, fusca (subatrobrunnea), lamellis mediis spiniferis ornata; anfr. $4^{1} / 2$, convexi, medio (ultimus supra medium) cari- 
nato-angulati. ultimus major: apert. rotundata. forte albolabiata. Jat. $2-2^{1}, 2$ alt. $2-2{ }^{1}{ }_{2} \mathrm{~mm}$.

Hab. Suecia in plerisque prorinciis a Skine usque ad Stockholm (ad Nackanäs, in Termdön et ad Kungshamn) sparsim et plerumque skane. Blekinge et fide E. N. ad Dalbrö in Södermanland exceptis) raro. - Dania in plerisque silris.

Tar. sutheris $\pi$. Exposé crit. 1011; t. costis obsoletis, spinis destituta, anfr. $\dot{f}$, apert. Iabro roseo. - Suecia in Blehinge (in faginea ad Pehrsbore* pr. Ronnebr: et Gotland skälsö). - Norr. ad Brerik. - Dania in Sjælland (ad Holsteinborg) et Jrlland (Linaa Westerskor'; ubique fide H. Lrnge. - Occurrit subfossitis in paludibus turfosis ad Stenstorp in pror. Skåne Sueciæ (unde specimina misit liber baro Cl. Kurck).

H. harpa Say Exped. St. Peters, 1824). T. orato-turrita. virescens $r$. corneo-lutescens. confertim striatula et distanter costulata; anfr. $4-5$. rotundati, ultimus magnus, rentro. sus; apert. rotundata, non labiata. Lat. $2-2^{1}{ }_{2}$, alt. $3^{1} 2$ -t́ $\mathrm{mm}$.

Hab. Suecia in Lapponia tota multis locis frequens. unde descendit rarior in Jemtland, Herjedalen, Dalarne (prope templum par. Lima: C. G. A.). Gestrikland (ad Gefle: Hj. Theel. Westmanland (ad Lindbo: C. H. J.) usque ad Stockholm (Stäket: O. S.. Lidingön: P. de L.. Bellerue: T. L.). - Torr. rerosimiliter tota. precipue in regionibus borealibus usque ad Taranger. - Femia tota, forte parte merid. - occidentali excepta: J. Sahlberg.

Subgen. 3. Gonostoma Held. T. aperte umbilicata. orticulata. discoidea, aretispira; apert. revticalis. triangularis. peristomate sepe bidentato.

H. obvoluta Müll. (Verm. Hist. II, 117'́). T. brunnea, pilosa. supra medio sæpius immerza. Lat. 12, alt. $5 \mathrm{~mm}$. Tariat edentata et glabra.

Hað. In Dania, ubi olim vixit. in tumulo antiquo in possessione Taareborg prope Korsör in Sjælland 1869 inrenta (C. II. P.) nec non mortua in Aerö.

Subgen. 4. Chilotrema Leach. T. aperte umbiticata, depressa. acute carinata: apert. tiansierse oualis, peristumate continus, so?uto. 
H. lapicida L. (Esst. nat. X, 1758). T. lenticularis, granulata. corneo-brunnea. fusco-maculata. Lat. 17-21. alt. 7 - 9 mm. -- Testa junior albida $=$ Helix albella $\mathrm{L}$.

Hab. Suecia a pror. Skâne usque in Dalarne et Medelpad (fere ad $63^{\circ}$ l. b.) sparsim occurrit. in rerionibus calcareis sæpius frequentissima. in forma primitiva aut rara aut ignota. Torv infra $61^{\circ}$ I. b. variis locis frequentissima. - Dania permultis locis rulgaris, multis deest. - Fennia merid. (ex. gr. Aland, Abo, Hogland et Ruskeala).

Forma meitelpatensis Cless. Malak. Blätt., 10-9. t. minor. subplana. anfractubus subtus conrexioribas: lat. 17. alt. $3^{12}$ mm. - Suecia in Medelpad ad Gaitström: C. G. An Aersson?

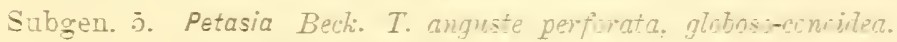
arctispira: apertura trisinuatiolunatr, obliqua. birlentata.

H. bidens Chemn. Conch. Cab., 17=03. T. corneobrunnea. granulato-striata; anfr. 6-6. ultimus albovittatus. Lat. s-9. alt. 5-b $\mathrm{mm}$.

Hut. Suecia in Skane late distributa, in Blekinge tantum ad limites scanenses, de cietero in Gland et Gotland et in plerisque provinciis usque ad Westmanland et Lpland ad tabricam Forsmark: P. de L.). sed sparsa et ubique rara. - I tnic in insulis fere ubique. in Jylland ad Aalborg. Tariat raro alba. - Fennia in Aland in par. Eckerö (A. J. Mela et in par. Birthala sp. jur. 15.59 inventum Mus. Helsingé.) - Helix fulra Mïill. p. p.!

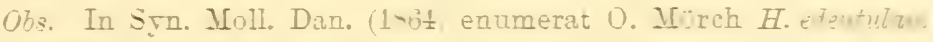
Drp. inter Mollusca danica, certe perperam. T. conoidea. cornea, peristoma intus labiatum subientatum. Diam. s mm. Tnum exemplar in Tegelverksshoren al Nrborg in ins. Fren ditate 1037 vacum et paullo detritum Lassen, in Holstein Dr. Müller.: Hec determinati erronea rstimanda sit et diagnosi et loco. H. edentula Drap. est species prorsus alpina.

Subgen. 6. Trichia Hurtw. T. umbiliata vel perf rata. glat a -

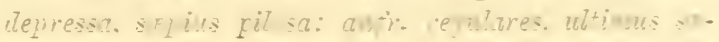

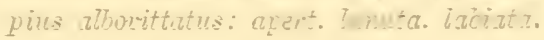

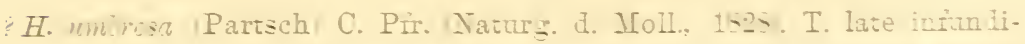
buIiforme umbilicata, depressa. vix conrexiuscula, tenuis, fallide 
cornea, striatula et sub lente densissime granulata; anfr. 5, lente accrescentes, ult. obtuse angulatus; apert. transverse ovalis, peristomate expanso. Lat. 11-13, alt. $6-6 \frac{1}{2} \mathrm{~mm}$.

Hab. Dania in Sjælland prope Holsteinborg (ubi O. Mörch dicit unum exemplar in hortu ad Bisserup lectum esse). Certe casu quodam inventa et non indigena faunie.

H. gyıata W. (Exposé critique, 1871). T. dilatate et late umbilicata, umbilico perspectivo, depressissima, fere plana, ferruginea v. obscure brunnea, pilosa; anfr. tardissime accrescentes, ultimus vix major; apert. rotundata, margine basali arcuato, leviter labiato. Lat. $8 \frac{1}{2}-10$, alt. $4-5 \mathrm{~mm}$. Hab. Dania ad Möens Klint.

Forma convexa.

H. hispida L. (Syst. Nat. $X, 1758$ ). T. umbilicata, depressoconvexa, corneo-brunnea, siepius vittata, hispida; anfr. sat lente accrescentes, ultimus compresso-rotundatus, subtus planulatus; apert. lunaris, depressa, margine basali strictiusculo, intus labio elevato. Lat. 7-8, alt. $5 \mathrm{~mm}$.

Hab. Suecia in provinciis merid. et mediis saltem usque ad $60^{\circ}$ lat. bor. passim, multis locis vulgaris, multis (ut in Blekinge, Smolandia montosa, Nerike etc.) rara v. rarissima, desuper etiam in regionibus multis et latis omnino deest. Norv. merid. vulgaris. - Dania tota. - Fennia meridionalis (Helsingfors, Åbo, Ladoga-Karelen) et in Lapponia in par. Kittilä specimen unicum J. A. Sandman invenit (K. M. L.).

Var. concinna Jeffr. (Brit. Conch. 1, 1862): t. sensim dilatate et demum sat late umbilicata, clepressa, pallida, raro-pilosa, anfr. arcti, peristoma margine basali leviter curvato, forte labjato; lat. 8-9, alt. $4 \frac{1}{2}-5 \mathrm{~mm}$. - Suec. in Skåne (Lund), Småland (Jönköping), Westergötl. (Hunneberg), Westmanl. (Westerås), certe etiam in provinciis plerisque cæteris. Dania in Sjrelland (Rude, Tuursö etc.), Fyen (Nyborg) et Jylland (Husum); regnum totum fide H. Lynge.

Var. nebulata Mke. (Syn. Moll., 1823): t. forte depressa, albida, peristomate forte labiato; lat. $71 / 2$, alt. $41 / 2 \mathrm{~mm}$. - Suec. in Kinnekulle.

Var. septemtrionalis Cless. (Jahrb. Mal. Ges., 187. ́): t. minor, umbilico angustiore et spira altiore. - Suecia a pror. Skăne ad Gefle passim. - Dania tota (H. L.). 
Var. depilata C. Pfr. (Naturges. I, 1S21): t. apertius umbilicata, parum convexa, nitida, omnino depilata, anfr, angusti, apert. depressa, margine basali stricto labiato cum columellari brevissimo angulum formante. - Suec. in Skine (Lund et Kristianstad), - Norv. ad Kristiania (ad Kongsberg). Dania ad Veile in Jylland.

Var. conica Jeffr. (Brit. Conch. I, 1862): t. parva, spira elevata, rotundato-conica, anfi. 5-6\% arctiores. - Suec ad Stockholm (Drottningholm: P. de L. et J. W.) et Upsala (in Horto botanico: P. T. C.). - Dania tota, fide H. Lynge. Var. sepulcrom W. (H. rufescens forma et H. hispida v. montana W. olim): t. depresso-globosa, convexa, fuscobrunnea, anfr. primi lente, ultimi celerius accrescentes, apert. rotundata, margine basali curvato, leviter labiato; lat. 9-10, alt. $61 / 2-7 \mathrm{~mm}$. - Suecia ad Kalmar (in sepulcreto vetero extra urbem*). Forte huc H. hispida forma in Noregic ad Kristiania, juxta viam ad Oscarshall: E. v. Martens. Forma ad plures species spectans.

Var. tardigyra W. (Verh. zool.-bot. Ver. Wien, 1892): t. infundibuliforme umbilicata, convexa, fuscobrunnea, striata, setis brevibus, arctispira, anfractu ultimo vix majore, apert. margine basali substricto, forte labiato; lat. 6 , alt. $4 \mathrm{~mm}$. Snecia in Gotland (ad Othem*) et Skine (ad Lund).

Var. mörchi W. (Fauna Binnenconch. Suppl., 1890): t. subtus dense spiraliter lineata. - Islandia (in horto ad Thorshamn).

H. liberta W. (Exposé crit., 1871). T. anguste et ad partem obtecte perforata, depresso-globosa, corneo-fusca v. rufescens, primum pilis raris brevibus munita, demum glaberrima; anfr. celeriter accrescentes, convexi, penultimus ter fere latior quam pracedens, superne profunde incurvus, ultimus magnus, vittatus; apert. rotundata, leviter labiata. Lat. 6--7, alt. $4-5 \mathrm{~mm}$.

Hab. Suecia in horto quodam ad Lund Scanize.

Obs. 1. Helix ab auctoribus germanicis et gallicis pro liberta sumta et descripta (et a me ipso in Fauna Binnenconch. II. p. 56 perperaur $_{+}$cum vera confusal alia forma est, quam sublibertam in schedis nominavi. H. liberta vera $a b$ hac et $a b$ omnibus affinibus anfractu suo penultimo lato priecipue distinguitur.

Obs. 2. "H. sericed Drap.? T. flavescens setis longis apice incurvis sat remotis. Dm. $8 \mathrm{~mm}$. Animal flavescens. H. hispida r. Alten (Erd- u. Fluss. Conch. Angsib, 1812 p. 4 t. 3 f. 6, H. hispida p. p. 
Miill. Verm. Hist. p. 74). Nonnulla specimina in Ordrupsmose et in Dyrhaven (Selandia).» Mörch Syn. Moll. Dan. p. 18. Quid sit nescio.

H. tumescens IV. (Öfv. K. Vet. Ak. Förl., 1881). 'T. anguste umbilicata, subcostulato-striata, rufobrunnea, non vittata, pilis brevibus dense hirsuta; anfr. regulares, ultimus major, rotundus, subtus convexo-tumidus; apert. rotundata, basi forte arcuato, intus circumcirca labiata. Lat. 7-8, alt. $5 \mathrm{~mm}$.

Hab. Suecia ad Stockholm (Sirishof *: C. G. A., Carlberg, Djurgården, Waldemarsudde: J. W., Drottningholm: P. de L.), Upsala (in Horto botanico: P. T. C.), Gefle (E. C.).

H. corasia mh. T. subperforata, convexa, apice prominente, rufobrunnea, zonula albida lata paullo supramediana in anfr. ult. cincta, pilis brevibus apice curvatis albidis dense obsita (postea pilis raris sublavigata); anfr. 5, regulariter accrescentes, convexi, sutura sat impressa, ult. compressorotundatus, ne minime angulatus, peripheria convexus, infra vix convexior, antice lente descendens; apert. lunato-rotundata, elabiata, peristomate tenui, recto, margine colum. superne late dilatato et reflexo; umbilicus intus angustissimus, infra duplo dilatatus, semiobtectus. Lat. fere 6 , alt. f. $4 \mathrm{~mm}$.

Hab. Suecia in Öland (Borgholm, inter lapides calcareas in declivitate boreali infra ruinas arcis: Gunhild Westerl.).

H. rubiginosa Z. ap. Schm. (Zeitschr. f. Naturw., 1853). T. umbilico angusto cylindrico, globosa, tenuis, rufescens v. fusca striata, pilis minutis albis apice curvatis sæpius dense obtecta; anfr. 41/2-5, sat lente regulariter accrescentes, convexiusculi, ultimus vittatus, subtus convexus; apert. rotundata, peristomate ubique simplici, margine columellari superne dilatato, reflexo. Lat. 5, alt. $3 \mathrm{~mm}$.

Hab. Suecia in insulis Öland (ad Kohlstad prope Borgholm) et Gotland (unde specimina in Museo Visbyensi asservantur).

Subgen. 7. Euomphalia W. T. perspectire umbilicata, depresse globosa. albido cingulata, apertura subcircularis, remote labiata. 
H. strigella Drap. (Tabl. Moll., 1801). T. cinereo-cornea, striata, fugacissime pubescens; anfr. ult. antice descendens. Lat. $13-14$, alt. $9-10 \mathrm{~mm}$.

Hab. Suecia sparsim a prov. Skâne usque in meridionalem partem prov. Dalarne. - Norv. meridionalis. - Dania tota passim, suis locis sirpius frequens. - Fennia meridionalis.

Forma minor Rossm. (Icon. f. 438), t. minor, pallida. tenuis, pellucida. - Suec. in Skine (ad Söndraby pr. Kristianstad: A. Nalm) et in Öland.

Var. subglobosa W. (Exkurs. Fauna, 188 i): t. umbilico angustiore et spira altiore. - Suecia rarior.

Var. colliniana B. (Noll. nouv., litig., 1863): t. umbilico latiore et infundibuliforme, anfractubus pulchre rotundatis, lentins et magis regulariter accrescentibus, apertura minore et labio fortiore; lat. 15, alt. $9 \mathrm{~mm}$. - Norvegia (locus specialis ignotus).

Sulfossilis (solida, glabra, spira planiuscula, umbilico latiore). Dania in strato calcis tophosæ in penins. Jylland.

Subgen. 8. Monacha Hartm. Testa subobtecte perforata. depresse subconoideo-globosa, minutissime granulata, vittata, apert. oblongu-lunaris, forte rufolatiata.

H. incarnata Müll. (Verm. Hist. II, 1774). T. rufescens; anfr. lentius accrescentes, ult. obtuse angulatus, antice deflexus. Lat. $12-13$, alt. $8-9 \mathrm{~mm}$.

Hab. Succia in Skåne sparsim, Blekinge ad Ronneby et Smaland ad Husquarna. - Daniu in plerisque silvis fagineis multo frequentior quam H. strigella.

Var. creticola Poulsen (in W. Fauna Suecica, 1873): t. umbilico magis a perto, albida $\mathrm{v}$. pallide lutea, nitida, pellncida, apert. depressa, labio roseo. - - Dania in Mröen.

Subgen. 9. Arianta Leach. Testa, obtecte perforata, depressoglobosa v. subconoidea, dense spiraliter lineata, plerumque fascia una fusea.

H. arbustorum L. (Syst. Nat. X, 1758). T. brunneo-lutescens, stramineo-aspersa; anfr. ult. ventricosus, antice siepius profunde descendens; apert. rotundata, peristomate patulo candide labiato. Lat. 20-23, alt. 17-22 mm. Hab. Succia in provinciis plerisque passim frequens sal- 
tim usque in Lule Lappmark $\left(67^{\circ}-68^{\circ}\right.$ l. b.), ubi fere ad terminum nivalem ascendit et vulgatior est supra regionem betulinam, sub forma alpicola, quam in vallis infrajacentibus. In multis regionibus vastis (ut in maxima parte prov. Blekinge et Westerbotten, circa Stockholm etc.) omnino desideratur. Norv. ubique frequentissima usque in Ostfinmarken. - Dania tota passim, suis locis semper frequens. - Fenmia meridionalis et media, usque ad Vasa et Paltamo (A. J. Mela).

Forma minor, lat. 17, alt. $12 \mathrm{~mm}$., t. colore typica, tenuis. Præcipue in regione boreali Scandinaviæ.

Forma morbosa-albina Rossm., t. pallide lutea v. flavoalbina, fascia obsoleta seu nulla, colore griseoflavescente sub epidermide tincta. - Suecia in Gotland et Skine (ad Lund).

Forma efasciata W., in regionibus certis prævalens.

Forma pallida W., lineis candidis. - Rarior.

Forma lutescens Dum. \& Mort., pallide flava, translucida, stramineo-marmorata, intus lacteo-marmorata, efasciata. - Rara forma in prov. subborealibus.

Var. calcarea Högb. (K. Sv. Vet. Ak. Handl,, 1841): t. calcarea, crassa, solida, fuscocastanea, luteomaculata, obscure purpurea intus; lat. 22-25, alt. $16-21 \mathrm{~mm}$. - Suecia fere ubique in Gotland et in insulis adjacentibus Karlsöarne. - Dania in ins. Möen.

Forma creticola Mörch (Syn. Moll. Dan., 1864), magna (lat. 27 mm)., persolida. - Dania.

Var. picea (Z.) Rossm. (Icon., 18337): t. tenuissima, transparens, fuscobrunnea v. castanea, unicolor v. subunicolor, apert. labio angusto tenui; lat. 15-18, alt. 12-14 $\mathrm{mm}$. - Suec. in Wermland (Trossnäs: amic. C. Fristedt). - Norv. in Österholm (Trondfjeld) et ad Kristiania. - Dania ad Lille Klint in Möen.

Var. septentrionalis Cless. (Regensb. Cor'r. Blatt, 1882): t. tenuis, sat depressa, distincte rimata, colore typica; lat. 20 -25, alt. 14--18 $\mathrm{mm}$. - Suecia a Skãne ad Medelpad rarior. - Norv. ad Skien.

Var. trochoidalis Roffirn 1868: t. alta, trochiformis; lat. 22, alt. $21 \mathrm{~mm}$. - Suecia passim. - Norr., Grötö.

Var. conoidca W. (Exposé crit., 1871): t. subperforata, conica, irregulariter subcostulata, fasciata et maculis pallide luteis ad lineas transversales densas confluentibus, anfr. 61/2; lat. $23-24$, alt. $25-26 \mathrm{~mm}$. - Suecia ad Lund.

Var. alpicole Fér. (Hist. géner. t. 27 f. 27 , t. 39 B. f. 4): t. alta, 
conoidea, solidior, flavida, distincte striata, subefasciata; forma minor, lat. 13--14, alt. 11-12 mm. (H. alpestris Z. ap. Rossm. Icon. 1835). - Suec. et Norv, in partibus borealibus passim.

Var. rutis Mïhlf. (Rossm. Icon. f. 78, 1837) aff:: t. sat aperte umbilicata, subcostulato-rugosa, spira planiuscula; lat. 20 -23, alt. $14-15 \mathrm{~mm}$. - Norv. ad Fredriksvrern. - Dania in Sjalland ad Leersöen prope "Lygten" et ad Rudesdal.

Var. depressa Held (in Chemn. Conch. Cab.) aff.: t. semiobtecte umbilicata, depressa, anfiactu ultimo fortius dilatato; lat. 23-25, alt. 15 - $15 \mathrm{~mm}$. - Suecia in Skine passim. Norv, ad Skien.

Var. Impmicola Mab. (Bull. Soc. Philom. Paris, 188.); t. globosa, depressula, solida, sat crassa, apice elato, flavida v. rufescens, immaculata, sæpius anguste fasciata, anfr. lente regulariter accrescentes, ult. rotundatus; lat. 17-18, alt. 13-14 mm., apert. 1. 9. a. $81 / 2 \mathrm{~mm}$. - Islandia borealis (fide clar. Servain).

Var: thamivaga Mab. 1. c.: t. globosa, tenuis, fasciata et maculata, apice rotundata, anfr. primi regulares, penultimus fortius accrescens, ult. roluminosus, supra cylindricus, infra ventricosus, apert. profunde excisa (1. $11 \frac{1}{2}$, a. $12 \mathrm{~mm}$.); lat. 20, alt, $18 \mathrm{~mm}$. - Dania (fide clar. Servain, ad quem O. Mörch specimina misit).

Var. foroeli B. ap. Serv. (Bull. Soc. Mal. Fr., 1889): t. rimata, conica, colore varia, anfr. superi sat celeriter accrescentes, nlt. magnus, cylindricus, apert. rotundata (lat. et alt. 11 mm.), margine colum. parum dilatato; lat. 22, alt. $18 \mathrm{~mm}$. - Dania (ficle clar. Servain, cui O. Mörch specimina communicavit).

Var. gotlandice W. (Nachr. blatt Mal. Ges., 1894): t. grandis, tenuis, pellucida, rufobrunnea, valde ventricosa, fasciata, parce leviterque maculata, anfr. ult. precipue subtus valde ventrosus, apert. magna, semicircularis, forte excisa, peristomate tenui, limbato; lat. 25, alt. $17 \mathrm{~mm}$., apert. 1. 13, a. $13 \mathrm{~mm}$. - Suecia in Gotland (ad Rosendal).

Var. celandica W. l. c.: t. semiobtecte umbilicata, pallide flavescens, dense maculata, intus lactea, apert, marginibus forte arcuatis, rectis, tenue labiatis, marg. colum. brevi verticali, superne dilatato-reflexo; lat. 20 , alt. $16 \mathrm{~mm}$. Suecia in Öland (ad Borgholm).

Subgen. 10. Eulota Hartm. Testa umbilicata, subglobosa, lineis densissimis spiralibus sculpta, scepe fascia 
5.t C. A. Westerhone, Synopsis Molluscorum Extramarinor. Seandin.

fusca, apert. subrotunda, intus tenue diffuse labiata.

H. fruticum Müll. (Verm. Hist. II, 1774). T. globoso-turbinata, rufescens v. lutescens; anfr. $6^{1 / 2}-7$, ultimus rotundus. Lat. 18-21, alt. 12-14 mm. - Variat rufa, pallide cornea et pallide lutea.

Hab. Succir a Skåne usque in Helsingland (ad Söderhamn, $61^{\circ}-62^{\circ}$ 1. b.). - Norv. meridion. et media. - Dania tota passim. - Fennia merid. et media usque ad Solovetsk (K. M. L.) et Kalkkiman ad Torneå (A. J. Mela).

Forma minor, lat. 14, alt. $11 \mathrm{~mm}$. - Jemtlaud (Brunflo: E. N.).

Forma conoidec W. (Exposé crit., 1871), t. conoidea, acuminata, alba; lat. 19 , alt. $20 \mathrm{~mm}$. - Suecia in Dalarne (acl Säter*: C. G. Andersson) et in Gotland (in juniperetis ad Sundre). - Fennia in Åland.

Forma ander'ssoni Cless. (Malak. Blätt XXV), t. minor, globosodepressa, spira planulata; lat. $17-18$, alt. $12 \mathrm{~mm}$. - Suecia in prov. Dalame (Risbergs grufra* et Osmundsberget) et Medelpad.

Forma insularum mh., t. anguste et margine columellari reflexo paullo obtecte umbilicata, conoidea, rufescenti-cornea, anguste fasciata, rarius concolor, epidermide sæpius detrita; lat. $15-16$, alt. $13-15 \mathrm{~mm}$. - Suec. in Blekinge (in insulis parvis maris baltici ex. gr. prope Risanäs*).

H. schrenki Midd. (Reise in Sibir. Il 1, 1851). T. minor, depressior, tenuis, diaphana, obsolete transversim et spiraliter lineata, subtus non inflata, typice corneo-albida, rufo-unifasciata; umbil. angustior; anfr. $5^{1 / 2}-6$, convexiusculi; apert. leviter v. vix labiata. Lat. 15 , alt. $9-11 \mathrm{~mm}$.

Hab. Sucia ad lacum Sarraure supra Qvickjock in Lule lappmark $\left(67^{\circ}-68^{\circ}\right.$ l. b.) specimina juniora in Aconito septentrionali invenit 1891 et benigne communicavit Prof. H. V. Rosendahl. - Norv?? Forte huc »Eulota fruticum», specimen semimaturum in Museo in Kristiania e Lofoten asservatum, de quo loquit ing. Birgithe Esmark in Tromsö Museums Aarsh. 1882. - Fennia merid. in regione montosa prov. Ryska Karelen (J. Sahiberg).

Subgen. 11. Xerophila Held. T. cornea, scepius calcarea $v$. porcellanea, plus minusve umbilicata, unicolor 
थ. fasciata et scepius subtus lineis fuscis pluribus, peristoma rectum.

† Striatella II. T. anguste umbilicata, depressoglobosa; anfractus ultimus rotundatus, obsolete angulatus; apertura rotundato-lunata.

H. striata Müll. (Verm. Hist. II, 177 1). Var. nilssoniana Beck (Index Moll., 1837). T. infra sat dilatate umbilicata, convexiuscula, irregulariter costata, opaca, crassa, albido-cinerea v. flavescens, unicolor, unifasciata v. multolineata; anfr. $11 / 2-5$; apert. non v. levissime labiata. Lat. $8-11^{1} / 2$, alt. 6-7 mm. - H. ericetorum Nilss.

Hal. Sreciu in campis quam maxime apricis et apertis, pracipue lapidosis in Westra Landtborgen insulac Öland, passim copiose.

? H. unifasciata Poir. Coq. de l:Aisne, 1801). T. anguste umbilicata, globoso-depressa, dense minute striata, albida, unicolor v. varie fuscofasciata; anfr. 5, convexiusculi, ultimus magnus, rotundatus, vix deflexus; apert. rotundato-lunaris; peristoma intus valide labiatum, margine basali rectiusculo cum columellari late patulo. Lat. $6-7$, alt. $4 \mathrm{~min}$.

Hab. Dania in ins. Fyen inter Nyborg et Holckenhavn, unicum specimen (fide O. Mörch). Valde dubia incola faunæe danicæ.

? H. conspurcata Drap. (Tabl. moll., 1801). „T. anguste umbilicata, depressa, subcarinata, corneo-cinerea, minutim variegata, peristoma rectum, simplex, diam. 5\%1/2 min." (Mörch); t. capillaceo-costulata, hispidula, anfr. $4-5$, depressi, apert. rotundato-lunaris; lat. $5-6$, alt. $31 / 2 \mathrm{~mm}$.

Hab. Dania in ins. Fyen, nonnulla specimina inter Nyborg et Holkenhavn (cl. Lassen fide O. Mörch). Etiam hæc meridionalis species ralde incerta est.

Obs. Potius hæc testa fuit Helix intersecta Poir. (H. caperata Mont.) ut etiam opinat clariss. E. de Martens (Sitz. ber. Berlin, 1882, testa quæ in Britannia et Belgia occurrit ut etiam ad Sonderburg in insula Als, prope ad Fyen, inventa est. Hæc opinio maximam probabilitatem obtinuit detectu hujus ipsius speciei nuperrime in Selandia meridionali.

H. intersecta Poir. (Coq. de l'Aisne, 1801). T. mediocriter umbilicata, depresso-globosa, confertim striata, sordide albida, fasciis corneis siepe interruptis ornata; anfr. 6, ultimus desuper visus penultimo vix latior, extus declivis, angulatus, infra convexior; apertura late lunata, albolabiata, 
labio insertionem marginis colum. non attingente. Lat. 8, alt. $4^{2} / 3 \mathrm{~mm}$.

Hab. Dania in Sjælland (ad Faxe Ladeplads, in plantatione abiegna parva mare versus: H. L., m. Julii 1896).

it Helicella Mor.-Tand. T. late perspective umbilicata, depressa; anfr. ultim. cylindricus; apert. subcircularis, lunate, intus labiata.

H. obvia Hartm. (Gasterop. Schweiz, 1840). T. in primis anfractubus dense striatula, plus minus distincte, saltem passim spiraliter lineata, candida, subatro-v. fuscobrunneofasciolata, strigata v. maculata; apert. rotundato-lunata, labiata; umbilicus mediocris, parum dilatatus. Lat. 14--16, alt. $8-9 \mathrm{~mm}$.

Hab. Norvegia exemplar unicum mortuum in silva ad Kristiania legit prof. Sars (fide E. de Martens), in Zirkonbrottet ad Fredriksværn specimina plura a. 1841 invenit C. M. Poulsen. Certe introducta.

H. ericetorum Müll. (Verm. Hist. II, 1774). 'T. levissime irregulariterque striatula, numquam spiraliter lineata, vix nitida, Iutescenti-albida, fasciis pallide hepaticis, rarissime interruptis; apert. parum excisa, sxpe subovata, parum labiata; umbilicus latus et forte dilatatus. Lat. 14 , alt. $7 \mathrm{~mm}$.

Hat. Dania in Sjelland inter Bistrup et Stavnsholt prope Fuursö, inter Frederiksdal et Birkeröd et ad Farum inventa.

Subgen. 12. Tachea (Leach) Gray. T. exumbilicata, subglobosa, fasciis 1--5 sapius distinctis; anfr. ult. tumidus; apert. perobliqua, late angulato-lunaris, margine basali stricto, calloso.

H. nemoralis Müll. (Verm. Hist. II, 1774). T. margine exteriore aperturæ nigrolimbato, pariete nigro, colore extus bene terminato. Lat. 20-23, alt. $16--17 \mathrm{~mm}$.

Hab. Suecia in Skåne sat vulgaris, preterea sparsim et plus minus raro, ut in Blekinge (ad Carlskiona), Smilland (ad Kalmar), Gotland (circa Wisby), Westergötland (Göteborg, Billingen, Kinnekulle), Södermanland (ad Hjelmaren: A. von Post), Stockholm (ad Haga et Drottningholm), tandem in Jemtland 
(Oviken in Storsjön: R. Hägg). - Norv. occ. passim frequens (ad Bergen, Hardanger, Stavanger etc.). - Daniu vulgaris. - Fennic ad Helsingfors (A. J. Mlela).

Forma creticola Mörreh (Syn. Moll. Dan., 1864), ponderosa, gigantea, albescens, umbilico interdum sat aperto; lat. 30 mm. - Snecia in Skrine ad Benestad. -- Dania in Möens Klint. *

Forma albolabiata W. (Fauna Europ. 1876), apertura labio roseoalbo v. albo. - Dania in Dyrehavskov * prope Nyborg, in Bornholm, ad Taarnberg prope Korsör.

Var. marginata W. (Exposé crit., 1871): t. peristomate brunneolimbato, intus albolabiato, margine columellari acuto, stricto, tenui, a margine exteriore incisura profunda disjuncto. - Suecia ad Kalmar (in sepulcreto vetero*), ad Malmö (in Möllevången).

Var. porrecta W. (Exposé crit., 1871): t. sutura antice recta, adeoque apertura magis rotundata. margine extriore supra fascias duas ultimas affixo. - Suecia ad Malmö (in Möllevången).

Variat colore ut: 1. lntea, 2. carnea, 3. hepatica, 4. lilacina, 5. incarnata (fasciis interruptis), 6. virescens (sutura alba), 7. castanea, \&e.

H. hortensis Müll. (Verm. Hist. II, 1774). T. peristomate aperture albolimbato et albolabiato, pariete cum testa concolori. Lat. 16--20, alt. $12-14 \mathrm{~mm}$.

Hiec testa certissime $H$. nemoralis L. est! Locus originalis est Ciaxa Oelandiee, ubi d. 15 Juni 1741 vidit et quem locum semper (in Syst. Nat. X et Fauna Suec. ed. 2) citavit (»Iter Oel. p. 127»). In tota hac insula, a me multoties et diligenter inquisita, H. nemoralis Müll. \& Auct. numquam inventa est!

Hab. Suecia per provincias omnes plus minus frequens saltem usque in prov. Dalarne. - Norv, meridionalis et media. - Dania tota. - Fennia meridionalis.

Forma minor Jeffr. (Brit. Conch. I, 1862), t. lutea, 5-fasciata v. unicolor; lat. 14, alt. $9 \mathrm{~mm}$. - Snec. ad Lutterhorn in ins. Fårö prope Gotland

Forma ludviciana "d'Aumont", Drt., t. minor, tenuissima, pellucida, membranacea, interdum fasciis 5 pallide luteis opacis. - Snecia ad Ronneby et in Jemtland (Oviken in Storsjön: R. Hägg') inventa. - Dania ad Frederiksdal et Linaa Westerskov; ubique fide H. Lynge. Islandia. 
Forma hybrida Poir. (Prodr., 1801), t. siepius rufa, unicolor, margine peristomatis interno carneo, violaceo v. subroseo. Suec. et Dania passim rarior. - Norv. ad Langesund et Brevik.

Forma crassa W. (Fauna Binnenconch. II, 1889), t. crassa, ponderosa, spira suhconica, anfr. ultimus parum descendens, apert. minor. - Suecia in Skåne (nt ad Benestad).

Var. perforata W. (Exposé crit,, 1881): t. lutea. 5-fasciata (fasciis 3 superis ad apicem productis), umbilico distincto. - Suec., ins. Gotland in juniperetis prope templum WVamlingbo.

Var. margineila C. G. Westerl. ap. W. (Fauna Binnenconch. Suppl., 1890): t margine columellari aperturæ acuto, stricto, tenui, a margine exteriore incisura profunda disjuncto. Suecia ad Kalmar (in sepulcreto vetero) et in Jemtland (Oriken ad Storsjön: R. Hägg). - Dania in ins. Möen.*

Subgen. 13. Pomatia Bcck. T. magna, globoso-conica v. ovatoglobosa; anfr. ultimus permagnus, inflatus; apert. magna, subverticalis.

H. adspersa Müll. (Verm. Hist. II, 1774). 'T. imperforata, ruguloso-malleata, griseolutescens, castaneofasciata, fasciis maculatis; peristoma late expansum et reflexum, incrassatum, album. Lat. 36-40, alt. 30--36 mm.

Hab. Dania in Kristiansvold ad Kjöbenhavn (H. Lynge).

H. pomatia L. (Syst. Nat. X, 1758). T. semiobtecte umbilicata, sat obsolete denseque spiraliter lineata, rufescens v. flavescens, siepius obsolete hepaticofasciata, globoso-ovata, spira brevis, obtuse conoidea, apert. rotundato-ovata, margine exteriore arcuato, columellari superne late reflexo. Lat. $35-40$, alt. $30 \mathrm{~mm}$.

Hab. Suecia in Skåne, multis locis copiosissima, Småland (ad Gransbo par. Säby), circa Stockholm et Upsala, nec non in Westmanland et Nerike (Nora) et aliis locis. - Norvegia ad Kristiania (fide Friele, false fide B. Esm.). - Dania fere tota. - Ubique olim a monachubus introducta.

Var. gesneri Hartm. (Gasterop. Schweiz, 1844): t. vix rimata, ovato-conica, solida, spira infra ventrosa, sursum conica, longitudinem aperturæ fere attingens, anfi. ultim. ad suturam longe profundeque descendens, apertura orata mar- 
Acta Societatis pro Fauna et Flora Fennica, XIII, n:o 7.

gine exteriore leviter arcuato; lat. 37 , alt. 4 h̆ $\mathrm{mm}$. - Suecia ad Wordsätra et Linnés Hammarby.

\section{Subfam. Pupina.}

Testa ovata $v$. clongata, nitidula, raro nitens; anfractus sapius lente v. reguluriter acerescentes; apertura longitudinalis, columella non tiuncata.

\section{Gen. Buliminus (Ehrenb.) Beck.}

Testa anyuste rimuta, ovato-oblonga; apertura ovalis, dimidiam longitudinem non superans, marginibus inaqua. libus, columellari stricto, reflexo.

B. montanus Drap. (Tabl. Moll., 1801). T. ovato-conica, fuscula, striata et minute granulata; apert. late ovata, limbo intus rufo. Long. 14-15, lat. $5 \mathrm{~mm}$.

Hab. Srecia ad Rosenlund prope Jönköping (J. E. Ketterstedt) et ad partem meridionalem montis Omberg (K. Ahlner).

B. obscurus Miill. (Verm. Hist. II, 177' 1, Helix). T. ovato-oblonga, fusca, striatula, non granulata; apert. ovata, limbo intus albo. Long. $8-10$, lat. $3^{1 / 2}-4 \mathrm{~mm}$.

Hab. Suecia in Skåne passim, Öland (Borgholm et Tveta), Gotland fere ubique, Småland (Rosenlund), Westergötland (Kimmekulle et Mösseberg), Östergötland (Omberg et ad Mjölsefall), Nerike (Hamrarne par. Göthlunda et ad Apelvik). - Norv. ad Langesund, Modum et Brevik. - Daniu tota. - Fennia (verosimiliter in parte merid.-orientali prov. Karelen, fide Nord. \& Nyl. in Finl. Moll.).

Var. oblongus IV. (Exposé crit., 1871): anfr. penult. precedentem subæquans, ultimus antice rectus, apert. parva; long. 11, lat. $3^{1 / 2} \mathrm{~mm}$. - Suecia in Småland (Rosenlund ") et Öland (Tveta).

\section{Gen. Pupa Drap.}

Testa cylindrica v. ovata, sapissime perobtusa; apertura parna $\left({ }^{1} / t-1 / 3\right.$ long. tot.), sapissime dentata, plas mimus iotundata, marginibus aqualibus. 
† Dextrorsa.

*) Apertura margine columellari suturecto.

1. Lauria Gray. T. ovato-cylindracea, rotundato-obtusa; apert. iamellis 1-2.

P. cylindracea Da Costa (Test. Brit., 1778, Turbo). T. fulvocornea, glabra, nitida; apert. plica parietali superne incrassata et cum margine exteriore connexa, peristomate plane reflexo, albolabiato. Long. 3-4, lat. $2 \mathrm{~mm}$.

Hab. Suecia in Gotland ubique frequenter. - Norv. in muris superioribus arcis Sverresborg ad Bergen et Lillesand (ad litora in Skoversöen frequens). - Dania in Sjielland, Möen, Bornholm et Jylland, passim frequens.

Forma inermis W. (Fauma europæa, 1876), apertura edentata. - Suecia in Gotland (Visby).

Var. anconostoma Lowe ap. Pfr. (Mon Heliceor. II, 18is) : apert. lamella parietali concolori, tenni. superne obsolete incrassata et a margine disjuncta, peristoma limbo angusto colorato; long. 4, lat. $2 \mathrm{~mm}$. - Suecia in Gotland (Visby).

Var. montigena W. (Exkurs. Fauna, 1884): t. minor, obscura, ventrosa, spira brevi rotundata, columella superne lamella transversa et intus denticulo conico. - Suecia in Skine (in monte Skäralid).

2. Torquilla (Faure-Big.) Stud. T. fusiforme-conica, acuminata; apertura lamellis $6-8$.

P. avenacea Brug. (Enc. meth. Il, 1792, Bulimus). T. rufobrunnea, substriata; anfr. 7-8, convexi; apert. 7-8 plicata: $2-2-3$ (h. e. 2 in pariete, 2 in columella et $3-4$ in palato), 1. \& 4. in palato parvi. Long. 7, lat. $2 \frac{1}{2} \mathrm{~mm}$.

Obs. Hre species eadem est ac Tubo musconum L. et descriptione ("testa magnitudine tritici, apertura ovato-acuminata") et e loco originali (Triberga borg in Öland, "Iter Oeland. p. 99", a Linné semper citato). Postea ab eo cum aliis speciebus, ut forte musconum Müll. et edentula Drap., confusa.

Hab. Suecia in insulis Öland et Gotland ubique vulgaris, in Westergötland (Kinnekulle) et Östergötland (Omberg). - $D a$ nia sinter lapides calcareas formationis transitoriæ Ins. Bornholm (Beck)» dicit O. Mörch in Syn. Moll. Dan. In Bornholm non occurit, affirmat H. Lynge in lit. 
Acta Societatis pro Fauna et Flora Fennica, XIII, n:o 7.

Forma paucidens W. (Fauna Bimnenconch. III, 1887), apertura plicis palat. solummodo 2. - Suec, in Öland et Gotland passim trequens.

Var. subcereana W. (Exposé crit., 1871): apert. 8-plicata: 2-2 - $i$ (his subrqualibus, marginem non attingentibus); long. $71 / 2-8$. lat. $21 / 2 \mathrm{~mm}$. -- Suecia in Öland (Borgholm).

Var. subhordeum W. (Fauma Binnenconch. III, 1887): t. ninor, at anfr. 7-8, lævigata, apert. 6-plicata: 2-2-2; long. 5, lat. $2 \mathrm{~mm}$. - Suecia in Öland (Borgholm).

+t) Apertura margine columellari curvato.

3. Pupilla Lcach. T. ovato-cylindracea, obtusa; apert. semicircularis, edentata $v$. denticulis 1-2 nodiformibus, labiata, marginibus expansinesculis.

P. muscorum Müll. (Verm. Hist. II, 1774, Helix). T. rufobrunnea, lavigata; anfr. 7-8, vix convexiusculi, ultimus antice parum ascendens et callo albido valido cinctus; apertura edentata. Long. $3^{1 / 2}$, lat. $1^{3} / 4-2 \mathrm{~mm}$.

Hab. Suecia »a Scania usque in regiones alpinas Norrlandice et Lapponize dicit Nilsson in Hist. Moll. Snec.; terminus borealis milii cognitus est Funnäsdalsberget in Herjedalen. Norv. tota usque in Ostfinmarken. - Dania tota. - Fennia usque in Lapponia rossica (K. M. L.).

Forma unidentata C. Pfr. (Gasterop, 1821), apertura denticulo obtuso profunde in pariete. - Typo frequentior.

Forma elongata Cless. (Excurs. Fauna ed. 1, 1876), longior, magis cylindracea, anfractubus 8 ; long. $31 / 2-41 / 3$, lat. 2 mm. - Suec. in Öland raro.

Var. bigranata Rossm. (Icon., 1839): apertura dente obtuso medio parietis (raro dificiente) et denticulo crasso nodiformi in palato. - Suecia multis locis (ut ad Romneby, in Mösseberg, ad Säter, in Lindeklint Gotlandiæ, ad As in Södermanland, ad Gefle et usque ad Östersund in Jemtland).

Var. lundströmi W. (Sibir. Moll., 1876): t. brunnea, nitida, anfr. 7, convexi, ultimus antice valde ascendens, callo transversali angusto concolori, apert. margine externo in pariete profundius affixo; long. $3 \frac{2}{3}$, lat. $1^{3} / \mathrm{t}$ mm. - Suecia ad Upsala (P. T. C.) et ad Östersund in Jemtland (E. N.). Norv. in insulis Lofoten* dictis, - Islandia.

4. Sphyradium (Ag.) Charp. T. cylindracea, raro subconoilea, apertura rotundata, edentata, peristomate acuto, simplici. 
P. edentula Drap. (Hist. Moll., 1805). T. cylindraceo-ovata, sublievis; anfr. 7, vix convexiusculi, 1-4 lentissime accrescentes, 5-6 latiores, subiequales, ultimus paullo altior et latior. Long. $2^{1 / 2}$, lat. $1^{1 / 4} \mathrm{~mm}$.

Hab. Suecia per totum regnum saltem usque ad Qvickjock in Lule Lappmark. - Norv. meridion. et media. - Dania tota. - Fennia meridion. et media vulgaris in silvis abietinis tenebrosis. Specimina matura (anfr. 7-8) ubique rarissima sunt.

Tar. turritella IV. (Malak. Blätt., 1875): t. conica, a basi attenuata, anfr. superi 5 lente accrescentes, nulli æquales. Suecia passim cum typo, sed multo rarior. - Fennia, Jakobstad.

P. inornata Mich. (Compl., 1831). T. omnino cylindrica, elongata, obtusa, subtilissime striata; anfr. 8, convexi, lentissime accrescentes, ultimis duobus æequalibus. Long. 4, lat. $1 \frac{1}{2} \mathrm{~mm}$.

Hab. Fennia ad Frugård (E. N.).

P. columella G. de Nits. (Würt. Fauna, 1830). T. cylindracea, distincte striata; anfr. 7, convexi, 1 \& 2 celeriter accrescentes, apicem obtusum formantes, $3-6$ æequales, ultimus ventrosus, magnus. Long. 3, lat. $1 \mathrm{~mm}$.

Hab. Suecia borealis saltem inter $67^{1} / 2^{\circ}-61^{\circ}$ lat. bor. et in Gotland (Klintehamn: E. N.). - Norv. usque ad limitem ultissimum (Vardö, ubi frequens: B. Esm.). - Fennia ad Jakobstad, Lyserholmen (Museum Helsingf.).

5. Isthmia Gray. T. minuta, cylintrica, gracillima, albirla, obtusa, anfi. 5. convexi, lente accrescentes, apertura rotundata.

P. costulata Nilss. (Hist. Moll. Suec., 1822). T. regulariter costulata et striata, pallide fulva (mortua alba); anfr. 6-7, ultimus antice callo albido valido transverso; apert. lamella parietali 1 , dente columellari 1 acuto et tuberculo ovali profunde in palato; peristoma intus valide albocallosum. Long. 2, lat. $1 \mathrm{~mm}$.

Hab. Suecia in Skåne (ad Esperöd), Blekinge (ad Sölvesborg), Östergötland (ad Spellinge par. Westra Harg), Cotland 
multis locis, Öland fere ubique copiosissime inter lapides calcareas et folia putrescentia. - Dania in Laaland (ad Knuthenborg) et Sjalland (ad Charlottenlund et frequens ad Faxe Ladeplads prope ad litora maris: H. L.).

P. odontostoma IV. (Fauna Europ., 1876). T. dense regulariter striata, pallide fulva; anfr. $6-7$, ultimus callo nullo v. obsoleto; apert. denticulo profunde in pariete et tuberculo guttiformi superne intus in palato; peristoma tenue. Long. $2^{1 / 2}$, lat. $3 / \pm \mathrm{mm}$.

$\mathrm{Hab}$. Norvegia in arce Akershus ad Kristiania.

P. minutissima Hartm. (Neue Alpina, 1821). T. dense regulariter striata, corneo-albida; anfr. 5-6, ultimus callo nullo v. obsoletissimo; apert. edentula; peristoma tenue. Long. 2, lat. $2 / 3 \mathrm{~mm}$.

Hab. Suecia in Nerike (ad pagum Kofta par. Glanshammar), Östergötland (Omberg), Södermanland (Dalbyö: Erl. Nordensk.), Westmanland (Westerås), Upland (Harg), Gotland (Visby et Hoburgen nec non in insulis Stora et Lilla Carlsön). - Norv. in Malmöen.

6. Alcea Jeffr. T. minuta, ovata, cornea $v$. brunnea, obtusa, sapius sublevigata, anfr. $1^{1 / 2}-6$, ultimus rotundatus, major, apert. semiovalis.

*) Cervir antice vix impressus, nec callo cinctus; apertura omnino elubiata.

P. genesii Gredl. (Tirols Conchyl., 1856). T. ovata, brunnea, nitida. lævigata; anfr. $4^{1} / 2$, tres ultimi lati, lente accrescentes, convexi, sutura profunda, antice ascendente disjuneti, penultimus subventrosus, ultimus ad basin postice constrictus, forte rectiuscule declivus; apert. edentula, margine exteriore superne forte angulato, infra subrecte obliqua. Long. $1^{2} / 3$, lat. $1 \mathrm{~mm}$.

Hab. Suecia in Westergötland (locus specialis ignotus) et in Jemtland (ad Östersund, $68^{\circ} 10^{\prime}$ 1. b. frecquens: E. N.) -- Subfossilem lib. baro Cl. Kurck hanc speciem in paludibus turfosis ad prædium suum Petersborg Scaniæ meridionalis (e determinatione prof. F. Sandberger) invenit. 
P. dicæa n. sp. T. cylindraceo-ovata, sub forti lente dense striatula, flavescenti-cornea; anfr. regulariter accrescentes, convexiusculi, sutura profundiuscula obliqua infra ascendente disjuncti, ultimus postice oblique subplanulatus, forte constrictus, antice utrinque dilatatus; apertura semiovata, edentula, pariete obliquo, margine exteriore regulariter arcuato, columellari dilatato, patulo. Long. $1^{2} / 3$, lat. $1^{1} / 3 \mathrm{~mm}$.

Hab. Suecia in Södermanland (Dalbyö: E. N.).

P. celata W. T. subperspective perforata, obesa, cylindraceoovata, obtusa, fusco-rufa, apice albida, sub lente dense striata; anfr. $5^{1} / 2$, convexi, sutura impressa, supra obliqua, infra horizontali disjuncti, tres ultimi lentissime accrescentes, antepenultimus pracedente fere duplo altior, ultimi duo fere requales, ultimus antice et postice fere reque altus, postice regulariter convexus; apertura semiovata, edentula, pariete subhorizontali, marginibus rectis, exteriore regulariter arcuato. Long. 2, lat. $1 \frac{1}{2} \mathrm{~mm}$.

Hab. Suecia in Södermanland (Dalbyö: E. N.).

obs. Hac descriptione accurata illa quæ errore singulari sub nomine celate in Nachrichtsbl. Mal. Ges. 1894 p. 173 data omnino deleta sit.

P. daliaca W. (Fauna Binnenconch. III, 1887). T. oblongo-cylindrica, rufescenti-cornea, nitidula, liuvis; anfr. 5, primi 2 parvi, 2. duplo brevior quam 4., ultimi tres subrequales, ultimus angustus; apert. dente conico valido superne in columella. Long. $1^{7} / 8$, lat. $1 \frac{1}{3} \mathrm{~mm}$.

Hab. Succia in Dalsland (locus specialis ignotus).

Var. dalecarlica n.: t. cylindrica, brunnea, vix striatula, anfr. $5-6$, lente regulariter accrescentes, ultimi duo subæquales, convexiusculi, ult. basi rotundatus, sutura medio obliqua, ad aperturam subhorizontalis, apertura semiovata, dente 1 parietali remoto brevissimo albo, 1 in fance fere ad basin, nodiformi, immerso, raro etiam denticulis $1-2$ punctiformibus fuscis obsoletissimis in palato medio extus, peristoma margine columellari subverticali, reflexo, exteriore superne forte curvato, deinde oblique rectiusculo, recto; long. $1 \frac{1}{2}-2$, lat. $1 \mathrm{~mm}$. - Suecia in prov. Dalarne (ad Leksand et ad Fu par. Mora: E. N.). 
P. pineticola W. (Exposé crit., 1871). T. ovato-cylindracea, lente attenuata, rufobrunnea, nitida, striatula; anfr. 5, convexi, sutura subhorizontali, profunda; apert. pariete forte declivi, inferne dente conico. Long. $2^{1 / 3}-2^{1 / 2}$, lat. $1^{1 / 2} \mathrm{~mm}$.

Hab. Suecia in Smiland (Tenhult ;), Westergötland (Borås: P. T. C.) et Westmanland (in loco Gröna gången ad Sala: P. T. C.).

P. gemma mh. T. cylindraceo-oblonga, supra medium lente attenuata, obtusa, castanea, nitida, sublævis; anfr. 6, convexi, tres ultimi lati, parum inxquales, superiores angusti (penult. precedente plus quam duplo altior), ultimus antice infra dilatatus, basi gibboso-cristatus; sutura supra obliqua, infra horizontalis; apertura (marginibus forte nondum omnino terminatis) subtriangulata, infra angustata, 1-2dentata: dente palatali nodiformi infra medium marginis, denticulo minutissimo supra medium; peristoma simplex, margine exteriore supra medium angulatim producto, superne forte arcuato, infra medium rectiusculo, columella recta, verticali ad basin incrassata, marg. columellari superne (pone columellam) dilatato et reflexo, infra columellam forte retrorsum arcuato; callus palatalis et cervicalis desunt; inter anfractum penultimum et ultimum linea alba transversalis, forte e labio tenui interno. Long. $2^{1} / 2$, lat. $1 \frac{1}{3} \mathrm{~mm}$.

Hab. Suecia in Södermanland ad Dalbyö (E. N.). Tantum unicum specimen hujus egregic speciei vidi.

P. ovoidea II. (Exposé crit., 1871). T. ventricosa, ovalis, medio lata, densissime striatula, nitida, corneolutea, pellucida, spira late conica; anfr. 5, sutura superne forte declivi, infra vix ascendente; apertura dentibus tribus minimis: 1 -1-1. Long. 2, lat. $1 \frac{1}{2} 2 \mathrm{~mm}$. Hab. Suecia in Småland (ad Tenhult).

P. arctica Wallenb. (Malak. Blätt., 1858). T. ovata, sublacrigata v. obsolete striatula, castaneo-rufescens v. fulvo-fusca, nitidula; anfr. $5-5^{1} / 2$ convexi, ultimus basi tumido-cristatus, sutura profunda, angusta, infra parum ascendente; 
apert. 2-3-dentata: 1-1-1 (0), dentibus concoloribus, brevibus, profundis, parietali minima, peristoma incrassatulum, margine exteriore ab insertione regulariter areuato, supra medium non producto. Long. $2^{1 / 2}$, lat. $1 \frac{1}{2} \mathrm{~mm}$.

Obs. In speciminibus lapponicis dens palatalis, semper perexiguus, plerumque prorsus abest; in specim. e terris magis meridionalibus dentes palatales sæpe 2, distantes, sed sæpius obsoletæ: forma quadividentata.

Hab. Suecia in Lappland et Herjedalen multis locis, in Södermanland (ad Mölby prope Gnestad: Erl. Nordenskiöld), Stockholın (ad Nackanäs: Hj. IV. \& E. N.) nec non in Småland (ad Tenhult). - Norv. in Ostfinmarken (usque ad Nordcap). Fennia ad Olenitsa et Tsjapoma in Lapponia Rossica (K. M. L.) Kalkkimaa, Kiiminki, Iisalmi (A. J. Mela) et certe aliis locis.

Var. extima W. (Sibir. Moll, 1877): t. late perforata, oratooblonga, brunneo-cornea, anfr. $51 / 2$, ult. antice callo pertenui concolori, apert. omnino edentata; long. $2^{3} / 4-3$, lat. $13 / 4 \mathrm{~mm}$. - Suecia, Jemtland ad Offerdal et Södermanland ad Dalbyö (E. N.) Antea in Sibiria ad Baklonowskij inventa.

P. alpestris Ald. (Trans. Soc. North. II, 1830). T. cylindracea, sub lente eleganter striatula, lutescenti-cornea, nitida; anfr. 5 , sutura sat profunda, medio descendente, infra bene ascendente; apert. 4 -dentata: $1-1-2$, dentibus albis (palat. sat longis, approximatis, lamelliformibus, parietali alto, conico-rotundato, semper præsente), margine exteriore superne in angulum obtusum curvato, supra medium angulatim producto. Long. $2^{1 / 4}$, lat. $1^{1 / 5} \mathrm{~mm}$.

Hab. Suecia per totum regnum usque in Lapponiam superiorem (ex. gr. ad Qvickjock, $67^{\circ}-68^{\circ}$ lat. bor.), plerisque locis copiose. - Norv. fere ubique usque in Westfinmarken. Dania ad Kjöbenhavn (Sorgenfri: H. L.) - Fennia tota frequens.

Forma mitis W. (Fauna Europ., 1873), apertura unidentata (1 -0-0), bidentata $(1-1-0)$, v. tridentata $(1-1-1)$. - Suec. in Skåne (Öfvedskloster), Blekinge (Ronneby) et Stockholm (Kungshamn et Vermdön: C. H., Haga: P. T. C., Stïket: J. W., \&c.), Jemtland (Östersund, Kall etc.: E. N.). - Norvegia passim cum typo. - Fennia ad Kantalaks in 
Lapponia Rossica (J. Sahlberg), Helsingfors et Härtonäs (K. M. L.).

Forma septentrionatis mh., t. haud parum major (ut P. arctica), fortius striata, dentibus palat. brevibus, concoloribus. Regio borealis et passim in Suecia media.

Var. shuttleworthiana Ch. ap. Pfr. (Mon. Heliceor, II, 1Sis): t. subovata, striatula, corneo-brunnea, nitidula, anfi. 5, sutura tenui, infra vix ascendente, apert. 4-dentata (1-1-2), dentibus concoloribus, palat. brevibus, sæpe minimis; long. $1 \%$, lat. $1 \mathrm{~mm}$. - Suec. in Skåne (Belteberga) et Upland (Dannemora: P. T. C.). - Dania in Sjælland (in stagno ad Ordrup: H. L.).

**) Cercix antice non callo cinctus; apertura intuss forte labiata, dens palat. labio adharens.

P. eremia mh. 'T. tumido-ovata, ventrosa, fusca, lavigata, anfr. ultimo antice rugoso-striata; anfr. 5, convexi, penultimus antepenultimo inulto major, ultimo extus ventroso paullo brevior; apert. subsemirotundata, 3-dentata, dentibus sat validis: 1-1-1, d. pariet. compresso, d. colum. conico, d. palat. crasso, tuberculiformi, callo v. labio albo forti adherente, peristoma marginibus callo cum callo aperturali adhaerente conjunctis, marg. exteriore supra arcuato, infra rectiusculo. Long. 2, lat. $1^{1 / 2} \mathrm{~mm}$.

Hab. Srecia in Medelpad ad Ànge (E. N.). Tantum unicum specimen vidi.

***) Cervix pone aperturam callo transversali cinctus, ante quem depressus.

a. - Dentes palatales liberi.

P. ronnebyensis W. (Exposé crit., 1871). T. oblongo-ovata, lufobrunnea, nitidissima, dense striatula; anfr. $5^{1 / 2}$, convexi, penultimus tertio $1 / 3$ major, ultimus in statu maturo callo pertenui concolori non impresso cinctus, sutura valde decliva, infra ascendente; apert. typice 4 -dentata: $1-1-2$ (dent. concoloribus, palat. immersis, brevissimis, longe discretis, d. parietali parvo, d. colum. minimo, acuto, rufo), pariete valde obliquo. Long. $2^{1 / 2}$, lat. $1 \frac{1}{2} \mathrm{~mm}$. - Variat dente colum. nullo et dentibus pariet. 2, minimis.

Hab. Saccia in Blekinge (ad Ronneby in faginea pr. Pehrsborg). 
P. lilljeborgi IV. (Exposé crit., 1871). T. ovata, ventrosula, castanea, v. obsolete dense striatula; anfr. 5, convexi, ultimus sulco lato non profundo inter callum superne tenuem basi gibbosulum rufum et aperturam; apert. 4-5-dentata: 11 (2, inferiore minimo) -2 dentibus palat. profundis, brevibus. altis), margine exteriore non v. vix impresso. Long. 2 $2^{1 / 4}$, lat. $1^{1 / 4}-1^{1 / 2} \mathrm{~mm}$.

Ha乙. Suecia in Skåne (ad Ringsjön), Blekinge (Ronneby prope Tresjön *, Långasjön etc.), Småland (Tenhult), Östergötland (Mjölsefall), Nerike (ad molinam prope Willingsberg). Dalarne (Långhyttan par. Husby), Jemtland (frequens ad Östersund: E. N.) Norrland, Lappland (ad Kurcoqueick, Strömnäs, PithoIm, Piteå, Lmeå). - Norv. in Börseöen pr. Skien. -Fennia ad Hirvilaks in Kuopio, Sääksmäki, Säïminki et Kannusjärvi (Mus. Helsingf.).

Var. merita W., apertura $4-5$-dentata (1-1-3 v. 2), dentibus minimis, nodiformibus, anfr. ultimus callo tenuissimo rufo v. perobsoleto, sulco nullo post aperturam. - Suecia ad Östersund in Jemtland (E. N.). - Fennia ad pagum Rissalanranta "(K. M. L.) Cfr. P. romebyens.

Var. globula n., t. subglobosa, ventrosissima, brunneo-castanea. striatula, anfi. 4, perconvexi, sutura profunda, penultimus maximus, antepenultimo triplo major, ultimo major et preesertim latior, ultimus antice dilatatus, callo transversali tenui concolori, postice sulculis parvis, apertura dentibus 4: 1 parietali parvo, profundo, 1 columellari ad basin columellæ, 2 palatalibus, punctiformibus, inter se distantibus. submarginalibus; long. 2, lat. $2 \mathrm{~mm}$. - Suecia ad Dalbyö in prov. Södermanland (E. N.).

P. antivertigo Drap. (Tabl. Moll., 1801). T. ovata, rentrosa. castanea, sublacvis; anfr. 5, convexiusculi, ultimus sulco angusto profundoque inter callum et aperturam; apertura typice 7-dentata: 2-3-2 (his lamelliformibus), margine exteriore supra medium forte impresso. Long. 2-21/2, lat. $1^{1} / 2-1 \frac{3}{4} \mathrm{~mm}$.

Hab. Succia per totum regnum (forte provinciis occidentalibus exceptis?) usque in Pite Lappmark. - Norv. ad Skien. Hvalöerne, Kragerö. - Dania tota. - Femia in insulis Aland in Kuopio, Karelen ife. 
Forma sexdentata Mont., apertura dente superiore columellari evanescente.

Forma octodentata Stud,, apertura dentibus palatalibus 3.

Forma ferox W. (Exposé crit., 1871), apert. 9-11-dentata: ? (3) $-3-4$ (2 elongati et 2 parvi) v. $3-3-4(5)$. - Passim raro.

Var. seminulum IV. (Exposé crit., 1871): t. minima, ovalis, anfr. 4, apert. 5-6-dentata: 1 (2)-2-2; long. $1 \frac{1}{2}$, lat. $1 \mathrm{~mm}$. Suecia in prov. Östergötland inventa.

b. - Dentes palatales aniice callo (labio) mofo interne adhorentes.

P. moulinsiana Dup. (Cat. Moll. Extramar. Galliæe, 1849). T. ovata, medio ventricosa (anfr. penultimus tertio $1 / 3$ altior et latior), levigata, rufocornea v. fulva; anfr. 5, convexi, ultimus antice callo albido valido cinctus; apertura 4 -dentata: $1-1-2$ (his brevibus), margine exteriore medio producto et leviter impresso. Long. $2^{3} / 4-3$, lat. $1^{3} / 4-2 \mathrm{~mm}$. Hab. Dania in Sjæelland (ad Veile Sö prope Holte).

P. gravida W. (Fauna Suecica, 1873). T. conico-ovata, basi ventricosa, sursum attenuata (anfr. penultimus tertio duplo latior et altior), virescenti-brunnea, striatula; anfr. $4^{1} / 2$, convexi, ultimus antice callo rufobrunnea; apertura 4 dentata: 1-1-2 (his lamelliformibus), margine exteriore non impresso. Long. $1^{3 / 4}$, lat. $1^{1 / 4} \mathrm{~mm}$. Hal. Norvegia ad Börsesö prope Skien.

P. pygmæa Drap. (Tabl. Moll., 1801). T. ovato-cylindracea, castaneo-rufa, levigata; anfr. 6, convexi, sutura infra recta; apert. semiovata, 5-dentata: 1 (altus)--2 (sup. conicus, inf. punctiformis, fere basalis) - 2 (breves, gibbosi), margine exteriore vix producto, vix impresso. Long. 11/2-2, lat. $1 \mathrm{~mm}$.

Hab. Suecia in plerisque provinciis saltem in Upland et Westmanland (Var. in Jemtland), in meridionalibus multo rarior quam in mediis. - Norv. meridion. et media passim. - D $D \ell^{-}$ nia sparsim. - Fennia ad Raseborg et Nordsjö.

Forma quadridens W. (Exposé crit, 1871), apertura dentibus $\dot{f}$ (dente columellari infimo defecto). - Cum typo, sed multo rarior, usque ad Östersund in Jemtland. 
Var. athesina Gredl. (Tirols Conch., 1856): anfr. ultimus pone apert. fere non impressus, apert. 6-dentata (scil. dente palat. supero tertio parvo). - Suecia in Skåne (ad Ringsjön). Södermanland (ad Dalbyö: E. N. et Âs: A. de P.), Stockholm (Lidingön ad Skäsätra: V. L.), Upland (ad Harg), Wärmland (ad Frossnäs: am. C. Fristedt) et ad Fleringe in Gotland (P. 'T. C.).

Forma bificla mh., apertura dente columellari superiore conico bifido v. geminato. - Suec. ad Dalbyö in Södermanland (E. N.).

P. substriata Jeffr. (Trans. Linn. Soc., 1830). T. breviter ovata, corneo-flavida, costulato-striata; anfr. $4^{1 / 2}$, convexi, ult. callo fulvido, medio impressus, sutura infra ascendente; apert. 6-dentata: 2-2--2. Long. $1^{3 / 4--2}$, lat. $1^{1 / 4} \mathrm{~mm}$.

Hab. Suecia in Skåne late distributa et sat vulgaris, de cretero sparsim et multo rarius per provincias fere omnes usque in Dalarne, Gestrikland (Gefle ad Lugnet et Hocksjöl: E. C.) et Jemtland (in Frösön: Coll. Cleveana, vulgaris ad Östersund: E. N.). — N'orv. ad Raaen, Langesund, Brevik et Molde. - Donic passim. - Fenmia ad Lahtis in Hollola (J. Sahlberg), Sääminki, Satakunta, Yläne et ad Nordsjö (Mus. Helsingf.), ad Träskïnda et Villnäs (E. N.).

Var. monas W. (Exposé crit., 1871): t. minutissima, oralis, fusca, anfr. $4 \frac{1}{2}$; long. $1 \frac{1}{2}$, lat. $1-1 \frac{1}{4} \mathrm{~mm}$. - Suecia in Blekinge (Ronneby*), Westergötland (Mösseberg) et Östergötland (Omberg). - Norv. ad Molde.

H Sinistrorsa.

7. Vertigo Miill. T. magnitudine et forma Subgen. Aliere aequalis.

P. pusilla Müll. (Verm. Hist. II, 177', Vertigo). T. elongatoovata, lrevigata, corneoflavida; anfr. $5-5^{1 / 2}$, convexiusculi, ultimus antice callo lato cinctus, basi paullo attenuatus; apert. semiovalis, 6-8-dentata: $2-2$ (4, inferioribus minimis) -2. Long. $21 / 2$. lat. $1 \frac{1}{4}-11 / 2 \mathrm{~mm}$.

Hab. Suecia a Skåne usque in Westmanland passim plus minusve frequenter, saltem usque ad Östersund in Jemtland (ubi rara: E. N.). - Norv. merid. et media. - Dania tota. Fennia meridionalis et media (Kuopio) late distributa. 
P. tumida IT. (Malak. Blätt., 1867). T. ventricoso-ovata, obtusa, basi ad dextrum tumida, fusca, striatula; anfr. $4^{1 / 2}$ -5 , convexi, ultimus antice pallidus, callo pertenui, basi compressiusculus: apert. late inæqualiter cordata, 4--6dentata: 2 (1)-2 (1) -3. Long. 17/s, lat. $1 \frac{1}{2} \mathrm{~mm}$.

Hab. Suecia ad Ronneby* et Borgholm. - Norv. in Malmöen. - Dania in Linaa Vesterskov.

P. collina W. (Exposé crit., 1871). T. ovata, cornea, striatula, spira brevi, subconica; anfr. $5^{1} / 2$, planiusculi, ultimus medio impressus, basi tumidus; apert. subcordata, tantummodo dentibus palatalibus 2 lamelliformibus. Long. 2, lat. 11/4 min.

Hab. Suecia ad Ronneby rarissima.

P. clevei IV. (Fauna Binneneonch. IIl, 1887). T. ventricosoovata; anfr. $5 \frac{1}{2}$, convexi, penultimus infra tumidus, ultimus altitudine aqualis, multo angustior; apert. truncato-ovata, margine exteriore medio stricto, obliquo, 2-dentata: 1-1 -0. Long. $1 \frac{2}{3}$, lat. $1 \mathrm{~mm}$.

Hab. Succia ad Sala (in loco Gröna gången dicto: P. T. C.).

P. angustior Jeffr. (Trans. Linn. Soc., 1830). T. ovalis, obtusa, infra attenuata, regulariter striata, fusca; anfr. 51/2-6, perobliqui, ultimus sulco longitudinali ad aperturam porrecto præditus; apert. triangulari-cordata, 4-dentata: 21-1 (longa). Long. $1^{7} / \mathrm{s}$, lat. $7 / 8 \mathrm{~mm}$.

Hab. Suecia (in Skåne tantum subfossilis in terra turfosa submarina ad Istad) in Gub. Calmar ad Böke par. Söderåkra, Blekinge (in ins. Werkö juxta Carlskrona, in Navalia regalia, Tvâgölingarne etc.), Bohuslän (Christineberg), Öland multis locis, passim frequens, Gotland multis locis, Westergötland (Mösseberg, frequens), Södermanland (Dalbyö: E. N. et Ås: A. de P.), Stockholm (Tullgarn: E. N.) et usque ad Östersund in Jemtland (ubi invenit am. E. N.). - Norv. ad Kristiania (in Lindöen \& Malmöen) et Asker. - Dania in Sjalland (Kjöbenhavn, Holsteinborg \&c.) et Jylland (ad Horsens: H. L.).

Var. producta IV. (Fauna Europæa, 1876): t. elongato-subovata, castanea, anfiactus convexi, ultimus postice rectus, per- 
obliquus, basi albogibbosus, sulco profundo subbipartitus, antice productus; long. 2, lat. $1 \frac{1}{4} \mathrm{~mm}$. - Suecia ad Borgholm.

Var. gothorum W. (Famna Europæa, 1876): t. breve ovalis, sat ventricosa, anfr. $41 / 2-5$, vix convexiusculi, ultimus medio impressus, basi parum gibbus, peristoma callo junctum: long. 1\% $1 \frac{1}{3}$, lat. $1 \mathrm{~mm}$. - Suecia ad Carlshamn (ad Bellerue*).

P. callista nov. sp. T. cylindracea, fusco-castanea v. rufobrumnea, dense striatula; anfr. 6, convexi, antepenultimus et præcedens omnino cylindracei, sutura profunda sejuncti, penultimus maximus, ultimus postice forte recteque decli. vis, medio sulco profundo transversali in duas partes divisus, unam majorem superam angustam cylindraceam et alteram perbrevem basalem forte tuberosam albam prope aperturam, ab hac sulco lato separatam; sutura perob liqua, ad aperturam non ascendens; apertura ad sini strum posita, subovalis, dentibus v. plicis subduabus, una breviore exteriore marginali, altera profunde intrante fere cum priore continua, plica palatali longa, intus forte curvata, columella callosa et labio calloso rufo profundo intra marginem exteriorem; peristoma rectum, margine exteriore superne forte arcuato et supra medium angulatim producto. Long. $1 \frac{1}{2}$, lat. ${ }^{2} / 3 \mathrm{~mm}$.

Hab. Suecia ad Dalbyö in prov. Södermanland (E. N.).

P. otostoma W. (Exposé critique, 1871). T. cylindrica, fulvobrunnea, striatula; anfr. $6 \frac{1}{2}-7$, convexi; apertura parva, auriformis, pariete valide obliquo, plicis palatalibus 2 lamelliformibus et denticulo infra in palato. Long. $2^{2 / 3}$, lat. $1 \frac{1}{3} \mathrm{~mm}$.

Hab. Suecia in Småland (ad Tenhult in collo Augustenburg dicto cum P. arctica, P. pineticola et P. ovoidea).

\section{Gen. Balea Prid.}

T. sinistrorsa, turvito-conica, apert. semiovalis, subpiriformis, vix ${ }^{1 / 5}$ longitudinis totius attingens, pariete superne extus tuberculo munito $v$. sublamellato.

B. perversa L. (Syst. Nat. X, 1758, Helix). T. striatula, niti- 
dula; anfr. $9-10$, convexiusculi, ultimus costato-rugosus, ad basin angulato-rotundatus; apert. ovato-piriformis, tuberculo parietali munita, marginibus callo tenui junctis. Long. 8-9, lat. $3 \mathrm{~mm}$.

Hab. Suecia in ins. Gotland et Öland vulgaris, passim in Wester- et Östergötland, raro in Blekinge (Ronneby etc.), Småland ad Kalmar (in vallibus arcis et urbis frequens), Nerike (in monte Ullaviklint par. Kil) et Westmanland (ad Vesterås). Norv. meridion. et media, passim. - Dania tota, passim. Fennia ad Nordsjö par. Helsinge et in Åland (ad Kastelholm, var. quadam f. Nord. \& Nyl.).

Var. suecana W. (Fauna Europ., 1878): t. turrita, a basi sensim longe attenuata, dense striata v. striatula, vix nitidula, anfi. 10-11, planulati, apert. piriformis marginibus sæpe callo conjunctis, interdum peristoma solutum), pariete fere in medio forte tuberculato; long. $9-101 / 2$. lat. $21 / 2-3 \mathrm{~mm}$. Suecia in Gotland vulgaris (1. or. in muris urbis Visby *) in Öland rara, in Westergötland (Kinnekulle), in Småland (Kalmar. - Norv. in ins. Ormöen et Malmöen in sinu Kristianiafjord.

Var. rayiana Bourg. (Rev. et Magaz. Zool., 1857): t. brevis, obesa, conico-turrita, sericina, olivaceo-cornea, confertim striata et strigillata, anfr. 8-9, ultimus extus ventrosus, basi rotundatus, apert. rotundata, denticulo minuto ad marginem; long. $7-71 / 2$, lat. $3 \mathrm{~mm}$. - Suecia in Blekinge (in horto in Carlskrona), Bohuslän (ad Lysekil: P. T. C.). -Norv. ad Fredrikshald.

Var. lucifuga (Leach) B. l. c.: t. elongata, conico-turrita, nitida, dense forteque striata, olivaceo-cornea, antr. 7-8, convexi, sutura profunda, apert. rotundata, marginibus longe sejunctis, vix callo pertenui connexis, tuberculo minuto prope marginem; long. 6 , lat. $1 \frac{1}{2} \mathrm{~mm}$. - Suecia cum typo, sed multo rarior a Skåne usque ad Stockholm (Kungshamn frequens, Wermdön, Lilla Björknäs, Stora Angsviken etc.) - Norv. ad Frederikshald.

Tar. polita W. (Fauna Europ., 1878): t. anguste subperforata, obscure cornea, polita, regulariter striata et crebro strigillata, anfi. \&, convexiusculi, sutura protunda, apert. oblongo-semiovata, tuberculo minuto; long. 7, lat. $21 / 3 \mathrm{~mm}$. Suecia ad Rommeby (ad Risanäs in muris frequens).

Var. pirostona W. (Nachrichtsbl. Mal. Ges., 1893): t. rimato- 
perforata, striatula, raro-strigillata, cervice fortius striata, apert. piriformis, subsoluta, marginibus continuis, lamella supera brevi et alta loco tuberculi aliarum formarum et sinulo instar Clausiliarmm distincto; long. 7, lat. $2 \mathrm{~mm}$. Suecia in Halland (ad Frillesås: A. J. J.).

\section{Gen. Clausilia Drap.}

T. sinistrorsa, fusiformis, apertura piriformis, $(1 / 5-1 / 6$ long. totius), semper lamellis, plicis et in fundo ossiculo elastico singulari munita.

+ Lamella spiralis a lamella supera disjuncta.

:) Lunella nulla; clausilium apice emarginatum.

1. Clausiliastra Möllend. T. sublcevis; peristoma continum, connexum; plicce palatales 3-6, incequales.

Cl. Iaminata Mont. (Test. Brit., 1803, Turbo). T. rufocornea. nitida, cervice depressa; anfr. 10-11, convexiusculi; apert. ovali-piriformis, plicis palat. 3 (1. 3. sat longi, 2. brevissima, 3. remota). Long. 17 , lat. $4 \mathrm{~mm}$.

Hab. Suecia passim a Skåne usque in Dalarne (ubi hinc inde frequens) et Helsingland (Arbrå). - Norv. meridion. et media saltem ad $64^{\circ}$ lat. bor., passim frequens. - Dania tota vulgaris. - Fennia meridionalis passim.

Forma minor W., t. pallide cornea, long. 12, lat. $3 \frac{1}{2} \mathrm{~mm}$.

Forma albina Pfr., t. virescenti-albida, pellucens. - Suecia raro (in Skåne et ad Jönköping).

Forma castanea W. (Mon. Claus., 1878), t. obesior, apert. perobscura, lamellis et plicis tenuibus, callo obsoletissimo. - Suecia raro (ex. gr. ad Stockholm *).

Var. plicifera W. (Exposé crit., 1871): t. ventrosula, rufobrunnea, apert. plicis palatal. $5-6$ (1. 2. 5. longis, 3. 6 . brevibus. 4. exigua, 1. 2. 5. 6. callo albo profundo adhærentibus (rel 1. longa, 2. 6. brevibus, 3. 4. exiguis); long. 16, lat. $4 \mathrm{~mm}$. - Suecia ad Lillö prope Kristianstad et in Öland ad Borgholm. - Dania ad Sorgenfri pr. Lyngby (H. S.). - Fennia in Karelia Ross. (J. Sahlberg).

Var. propinqua W. (Exposé crit., 1871): t. gracilis, longe attenuata, nitida, lutescenti-cornea, anfi. 12, superi convexi, plicæ palat. 4 (1. longa, 2. 4. breves, 3. exigua, 1. 4. cailo adhærentes); long. 19-20, lat. $3 \frac{1}{2}-4 \frac{1}{2} \mathrm{~mm}$. Suecia in Skĭne (Belteberga). 
Tar. pirostoma W. (Exposé crit., 1871): t. pallida, palato fusco, aufr. 11, plamulati, ultimus antice subplicata, peristoma subsolutum, plicæ palatales 4 ; long. 17, lat. $4 \mathrm{~mm}$. - Suecia in Skine in faginea ad Belteberga).

**) Lunella distincta; clausilium integrum.

2. Alinda Test. T. costulata; apertura basi canaliculata; plicre. palatal. superu 2 longa, plica suturalis tenuis.

Cl. biplicata Mont. (Test. Brit., 1803, Turbo). T. ventrosula; lamella infera horizontalis; lunella dorsalis v. subdorsalis, arcuata; plicie palat. extus valde divergentes, inferior longius producta. Long. $16--17$, lat. $4 \mathrm{~mm}$.

Hab. Suecia in Skåne (Esperöd, Öfvedskloster, Blommerröd), Blekinge ad Carlskrona. - Norv. in insula Manger prope Bergen. - Dania in Sjælland multis locis et (fide H. Lynge) in Jylland, in ins. Möen vulgaris.

Forma sordida Z., t. minor, anfr. 10-11; long. 12-13, lat. $3^{1 / 2}$ mm. - Suecia in Skåne (Öfvedskloster).

Forma armata W. (Exkurs. Fauna, 1881), interlamellare plicis 1-4 munitum. - Suec. ad Carlskrona. - Dania in Möen et (fide H. Lyıge) in horto ad Basnæs.

Var. austera W. (Exposé crit., 1871): lamella infera antice plerumque bifurcata, lunella lateralis valde arcuata; long. 15 -18, lat. $31 / 2$ mm., v. $191 / 2-3 \mathrm{~mm}$. - Suec. in Gotland (Hoburgen).

Cl. plicata Drap. (Hist. Moll., 1805). T. gracilis; lamella infera profunda, oblique ascendens; lunella lateralis, subrecta; plice palatales parallelæ; peristoma intus pliculis confertis circumdatum. Long. 14-18, lat. 3-4 mm.

Hab. Succia in Gotland (Hoburgen). - Dania in Sjælland (Fuursö), Möen et Bornholm vulgaris.

Forma implicata Bz. (Siebenb. Verh., 18כ̌1), t. peristomate, præsertim margine exteriore, implicato. - Cum typo, sed rarior.

Var. elongata Ad. Schm. (Rossm. Icon. f. 708): t. gracile elongata, subcylindrica, anfr. $15-16^{1} / 2$; long. ad 19 , lat. $3 \frac{1}{3}$ mm. - Suec. in Hoburgen rara.

it Lamella spiralis vudimentalis $v$. nulla, lunella valida usque ad suturam porrccta, clausilium integrum.

3. Papillifera (Hartm.) T. albida, sutura albopapillifera. 
CI. nilssoni W. (Exposé crit., 1871). „T. fusiformis, albida r. cinerea, tenuiter striata; anfr. 9, vertice obtuso, sutura papillis albis crenulata; apert. rotundato-ovata, patula, columella biplicata, interstitio plicarum et peristomate lavibus. Long. 11, lat. $3 \mathrm{~mm} . »$ (Nilss. Hist. Moll. Su., 1822: Cl. papillaris).

Hab. Suecia »in silvis frondosis, prosertim fagineis, ad Fröllinge in Hallandia reperta 1814» (Nilss.); Alibi et postea numquam visa.

tht Lamella spiralis cum lamella supera conjuncta (exc. Cl. sejuncta et connectens).

4. Cusmicia Brus. T. striata $v$. costulata, basi distincte cristata et sulcata, apert. basi intus canaliculata et callosa, plicee palat. superce 1-2 (1. longa, 2. breiissima $v$. nulla), lunelia brevis, claus. integrum.

Cl. parvula Stud. (Coxe Trav. 1789, Helix, nomen, \& Kurz. Verz., 1820). T. gracillima, cylindrico-fusiformis, subtilissime striatula ad lavigata, raro strigillata; lamella infera profuncla, raro antice subfurcata; plica palat. supera ultra lunellam valde arcuatam sat longe producta; callus tenuis plica (callus) basalis validus. Long. 9, lat. $2 \mathrm{~mm}$.

Hab. Dania in peninsula Jylland (in silva ad Marseliborg 2 specimina lecta fide 0 . Mörch).

Cl. dubia Drap. (Hist. moll., 1805̃). T. ventrosula, striata r. costulata, strigillata, spira brevi, lente attenuata; lamella infera perobliqua, stricta, retrorsum anguste bifurcata, antice bigibba; callus basalis tenuis. Long. 13, lat. $3 \mathrm{~mm}$. Hal. Suecia in Skåne (Belteberga), Blekinge (Ronneby, rarissime), Dalarne passim et Jemtland (ad Öresundet et Alsen). - Norv. ad Kristiania (et Srinvär?). - Dania in Bornholm (et Möen? Negat H. Lynge). - Fennia ad Mäntsälä.

Forma subspeciosa IV. (Fauna Suec, 1878), long. 14, lat. 3 mm. - Suecia in Dalarne. - Dania in Möen (Lille Klint* sat frequens).

Forma gracilis TT. 1. c., t. gracilis, cylindraceo-fusiformis, corneo-fusca, dense strigillata; long. $10-11$, lat. $21 / 4^{-2} 2^{1 / 2} \mathrm{~mm}$. - Suecia in Dalarne. 
Forma minor IV. l. c., t. typica, sed long. 10-11, alt. $2^{1 / 2} \mathrm{~mm}$. - Snecia in Dalarne.

Var. obsoleta Ad. Schm. (Krit. Grupp., 1857): t. ventrosa r. gracilis, fuscocerasina, lamella infera perobliqua, subocculta, callus palat. et plica subcolumellaris obsoleti. Suec. in Dalarne. - Norv. ad Asker et Bergen.

Tar. Lifurca n.: t. subventrosa, forte costata, nigrescentibrunnea, lamella infera antice dilatata, nee gibbosa, intus late bifurcata, ramis aque fortibus, callus internus margini parallelus, infra intus pliciformis; long. 12, lat. $2^{1 / 2}$ mm. - Suecia in prov. Småland ad Tenhult.

Cl. bidentata Ström (Trondhj. Selsk. Skrifter, 1765, Turbo). T. gracilis, densissime striatula ad sublevigata, parcius strigillata, spira lente attenuata; Iamella infera subhorizontalis, arcuata, sæepius retrorsum late bifurcata, antice furcata, v. deltoidea, callus basalis pliciformis, fortis, albus. Long. 9, lat. $2^{1 / 2} \mathrm{~mm}$.

Hab. Suecia ubique a Skâne saltem usque in Jemtland (Areskutan et Offerdil). - Norv. tota usque in Westfinmarken. - Dania tota. - Eennia verosimiliter tota.

Var. septentrionalis Ad. Schm. (Kirit. Grupp., 1857): t. curta, rentricosa, obscura, subtilius striatula; long. 8 , lat. $21 / 2 \mathrm{~mm}$. - Cum typo, sed rarior.

Var. exigua W. (Exposé erit., 1871): t. cylindraceo-fusiformis, subtiliter striatula, vix strigillata, apert. parvula; long. 8, lat. $2 \frac{1}{2} \mathrm{~mm}$. - Suecia meridionalis et Dania cum typo, sed raro. - Norv. ad Modum et Fiskum.

Var. subrugosa W. 1. c.: t. fusca, interdum crebro-strigillata, subtiliter striatula, anfi. ult. antice costulato, basi subbicristato, eristis sat validis, lamella infera sepissime simplex, intus arcuata, antice stricta, marginalis, subtus sæpiss. duplicata, interlamellare sæpe pliciferum, lunella valde arcuata, callus palat. fortis; long. 8-10, lat. $2 \mathrm{~mm}$. - Suecia in Öland (Borgholm *), et Gotland. - Norv., Modum, - Dania in Bornholm et Jylland (H. L.).

Var. erronea IV. i. c.: t. parva. plica palatalis supera ultra lunellam areuatam longissime producta. - Suecia in Skåne (ad Klinta* prope Ringsjön). - Norv, ad Modum. - Dania in Bornholm.

Tar. retracta n.: t. ventrosula, fusca, dense striata, in anfr. ultimo densius costulato-striata, lamella infera immersa, alta, arcuata, simplex, plica palat. supera ultra lamellam 
producta, callus basalis tenuissimus r. nuflus; long. 10, lat. $2 \frac{1}{2} \mathrm{~mm}$. - Fennia ad Villnäs (E. N.).

CI. cruciata Stud. (Kurz. Verz., 1820). T. ventrosula, sat distanter costulata v. costata, interstitiis transverse rugosis, longe strigillata, spira lente longecpue attenuata; lamella infera arcuata, horizontalis, retrorsum et sæpius antrorsum furcata; callus basalis distinctus. Long. 9, lat. $2^{1 / 2} \mathrm{~mm}$. Hab. Suecia in Jemtland (ad Östersund frequens: E. C. \& E. N.). - Norv ad Skovlökken prope Veiensten in Gausdal. - Fennia multis locis (ex. gr. Kuopio, Hiltulanlaks, Niemiskylä, Hirvilaks, Nordsjö, M̈̈ntsälä, Kuanlampi, Kivinebb, Walamo, etc.).

Cl. sejuncta IV'. (Exposé crit., 1871). T. ventroso-subclavata, costulata, spira brevi, celeriter subconcave attenuata; lamella supera a spirali disjuncta, infera arcuato-subhorizontalis, postice et sxpe antice bifurcata; plica palat. supera ultra lunellam producta; callus basalis tenuis vel nullus. Long: $12-13$, lat. $3 \mathrm{~mm}$.

Hab. Sueciı in Skåne, Östergötland, Westergötland, Nerike et Södermanland passim in regionibus petra calcarea substratis. - Norv. ad Ekeberg prope Kristiania et inter Skien et Porsgrund, rarissima. - Dania late distributa et passim frequens.

Forma major Ad. Schm. (Krit. Grupp., 1857); long. 131/2-15. lat. $3 \frac{1}{4}-31 / 2 \mathrm{~mm}$. - Suecia in Skåne (Belteberga).

Forma minor Ad. Schm. 1. c., long. 10, lat. $2^{3 / 4} \mathrm{~mm}$. - Suecia in Westergötland (Mösseberg).

Var. truncatula W. (Fauna Suec., 1878): t. corneo-Havida, aufr. planulati, sursum lente declivi, infra abrupti, peristoma incrassatum, productum. - Dania in Sjælland (ad Holsteinborg;.

Var. ommce W. (Exposé crit., 1871): t. cornea, costulato-striata, apert. piriformis, basi profunde canaliculata, peristomate libero, sinulo parvo depresso, lamella supera $\lambda$ formis. plica palat. supera ultra lunellam longe producta; long. 13. alt. $3 \frac{1}{4} \mathrm{~mm}$. - Suecia in Omberg:

Tar. personata IV. 1. c.: t. fere rufobrunnea, subtiliter costulato-striata, apert. parva, rhomboideo-ovata, marginibus callo conjunctis, lamella infera profunda, retrorsum fur- 
cata, antice bigibbosa, plica palat. supera ultra lunellam arcuatam longe producta; long. 11, lat. $3 \mathrm{~mm}$. - Suecia in Ski̊ne (Belteberga).

Deviatio plica palatali supera pollicari vel sesqui pollicari elongata, in gyrum testam circumcingente. - Dania in Lolland et in Sjælland ad Frederikslund prope Holte.

Cl. connectens $\mathrm{W}$. (Exposé crit., 1871). T. fusiformis, costulis eminentibus sinuatis æcualibus seulpta, spira attenuata; apert. basi profunde canaliculata, lamella supera parva, a spirali disjuncta, infera magna, simplex, plica palat. supera non ultra lunellam producta; callus palatalis distinctus. Long. 16, lat. $3^{1 / 2} \mathrm{~mm}$. - Facie C.l. biplicatam bene in mentem vocat.

Hab. Suecic in Skåne ad Westra Wram.

5. Pirostoma Vest. T. basi obsolete cristata et sulcata, apertura rotundato-piriformis, nec canaliculata nec callesa, de cutero ut Cusmicia.

Cl. ventricosa Drap. (Hist. Moll., 1805). T. ubique a equaliter costulata, ad suturam raro-strigillata, nigricanti-rufa v. brunnea, spira longe attenuata; anfr. 11-12, ultimus basi tumidus, subcarinatus, vix sulcatus; apertura rotundato-piriformis, lamella infera $\backslash /$ formis, interlamellare læve. Long. 17-20, lat. $4-4^{1} / 2 \mathrm{~mm}$.

Hab. Sucia in Skåne multis locis, Småland (ad Rosenlund prope Jönköping), Westergötland (Kinnekulle), Östergötland (Omberg), Dalsland (Dingelvik). - Norv. ad Brevik. Dania in Sjæelland frequens, Möen, Bornholm etc., nec non in parte orientali peninsulæe Jylland.

Forma tumida Ad. Schm. (Krit. Grupp., 1857), t. curta; long. 16, lat. $11 / 2 \mathrm{~mm}$. - Cum typo passim.

Forma gracitior Ad. Schn. 1. c., long. 171/2-19, lat. 32/3. mm. - Dania in Sjælland (Frederikslund pr. Holte) et Möen. Forma major W.; long. 22-23, lat. $4^{1} / 2-5 \mathrm{~mm}$. - Suec. in Skåne (Skäralid).

Cl. rolphi (Leach) Gray (Med. repos., 1821). T. confertim costulato-striata, anfractu ultimo latius costulata, cerasino-brunnea v. cornea, raro strigillata, spira breve attenuata; anfr. 9--11, ultimus crista et sulco sat distinctis; apert. rhom- 
boideo-piriformis, interlamellare sæpius minute plicatulum. Long. 12-15, lat. $3 \mathrm{~mm}$.

Hab. Novvegiu ad Kristiania (in arce Akershus) et Asker.

Cl. plicatula Drap. (Hist. Moll., 1805). T. costulata, brunnea, raro strigillata, spira attenuata; anfr. 10-13, ultimus basi transverse tumidus (ne minime cristatus vel sulcatus); apert. rotundata, lamella infera $\times$ formis, interlamellare pliculosum. Long. 12-13, lat. $2 \frac{2}{3}-3 \mathrm{~mm}$.

Haz. Suecia a Skåne usque in Dalarne (ad fodinam Bisbergensem et ad Spjutbo). - Norvegia meridion. et media. - Dania tota. - Fennia meridionalis.

Forma curta Ad. Schm. (Krit. Grupp., 1857), t. curta et rentricosa, long: 10-12, lat. 3-31/2 mm. - Suecia in Skåne (Belteberga). Östergötland (Omberg), Dalarne (Süter), $\mathrm{C}_{\mathrm{p}}$ land (Ekbyholm: E. N.). - Norr. - Fennia ad Ladoga (Mus. Helsingf.).

Forma major W. (Exposé crit., 1871), anfl. 14-15, apertura longa, piriformis; long. $17-19$, lat. $22 / 3-3 \mathrm{~mm}$. - Suecia in Skåne (Belteberga * et Skäralid), Östergötland (Omberg'), Westergötland (Falköping).

Forma labiosa Rossm.. peristomate valde incrassato. - Raro.

Var. fallaciosa W. (Exposé crit., 15:1): lamella infera antice truncata, bigibba, superne e medio ranis 2 valde diver. gentibus, interlamellare prorsus læve. - Suecia in Skåne (Belteberga).

Var. leucostoma W. l. c.: apertura, faux et plicæ lacteæ, plicæ interlam. 3-4 argutæ. - Norv. ad Drammen et Modum.

Var. extensa IV. (Monogr. Claus., 1878): t. gracilis, spira sensim valde attenuata, anfi. $14-15$, superi 6 subæquales, cæteri lente accrescentes, ultimus basi rotundatus, non gibbosus, sutura impressa, apertura oblongo-piriformis, lamella infera antice pliculis 2 divergentibus; long. 14, lat. $21 \% 2 \mathrm{~mm}$. - Suecia in Skåne (ad Belteberga).

Var. scanicu TW. (Exposé crit., 1871): t. subtiliter striatula, ceracino-brunnea, anfr. 9, ultimus antice costulato-striatus lamella infera profunda, valida, simplex, antice truncata, medio ligibba; long. 9, lat. $2 \mathrm{~mm}$. - Suecia ad Belteberga.

Var. oreas W. l. c.: t. distanter costulata, rufobrunnea, spira brevi, lamella infera antice longe dichotoma, plica palat. ultra lunellam longe producta, callus palatalis validus, albus; long. 13-15, lat. $3 \mathrm{~mm}$. - Suecia ad Belteberga. 
Var. cmule W. 1. c.: t. argute costulata, cervice obsoletissime cristato et sulcato, lamella infera remotissima, intus valde elata, simplex, antice abbreviata, pliculis 2 longe productis, lamella spiralis vix cum lam. supera conjuncta; long. 10, lat. 2-3 mm. - Suecia in Öland (Borgholm *), Westergötland (Mösseberg), Upland (Ekeby: E. N.), Gotland (Hallebras).

Var. cruda Z. ap. A. S. (Krit. Grupp. eur. Claus,, 1857, f. 50): t. ventrosulo-fusiformis, sat argute costulata, lamella infera simplex, antice non (vel obsolete) progrediens, interlamellare omnino lieve (rel obsolete uniplicatum); 1. 9-10, d. $21 / 2 \mathrm{~mm}$. - Suecia in Småland (ad Rosenlund prope Jönköping) et in Skåne (ad Belteberga). - Dania ad Sælsö: H. L.

Subfam. Stenogyrina.

$T$. ovato-oblonga $v$. elongata, polita, anfractus celeriter accrescentes, ultimus longissimus, apertura verticalis, anguste ovata, elongato-acuminata, $1 / 3-1 / 2$ longitudimis totius attingens, peristoma rectum, incrassatum.

\section{Gen. Cionella Jeffr.}

\section{Subgen. Zua Leach.}

T. ovato-oblonga, fusca, apertura $1 / 3$ longitudinis, tenue rufolabiata, columella brevis paullisper truncata.

C. (Z.) lubrica Müll. (Verm. Hist. II, 1774, Helix). T. fulvocornea, lævis; anfr. 6, convexi, ultimus basi rotundatus. Long. 6, lat. $2 \mathrm{~mm}$.

Hab. Suecia per totum regnum fere ubique, saltem in Lule Lappmark $\left(67^{\circ}-68^{\circ}\right.$ lat. bor.). - Norv. usque in Westfinmarken (saltem ad Tromsö). - Dania vulgaris. - Fennia tota usque in Lapponia Rossica (Imandra, Olenitsa, Varsuga, Tsjapoma: K. M. L.).

Forma nitens Kok. ap. Gallenst. (Kärnth. Conch., 1852), t. magna, firma, nitidissima, pallide castanea; long. 7-8, lat. $3 \mathrm{~mm}$. - Suecia in Blekinge (ad Ronneby, rarissime) et in Pite Lappmark (ubi sæpe inventa). - Fennia in Fuolajärvi Lappmark.

Forma ovata Jeffr. (Brit. Conch. I, 1852), t. ovalis, fuscobrun- 
nea; long. 5-6, lat. $2 \frac{1}{2}-3 \mathrm{~mm}$. - Suecia passim raro cum typo.

Forma viridula Jeffr. 1. c., t. subhyalina, splendida, vitrea, viridula. - Suecia in Skåne (ad Ringsjön) et ad Stockholm (in Wermdön).

Forma exigua Mke. (Syn. Meth., 1830), t. typo gracilior, oblongo-cylindracea, anfr. 5 , apertura angusta, peristomate forte albo-incrassato; long. 4--5, lat. $1 \frac{1 / 2}{-15 / 6} \mathrm{~mm}$. - Cum typo, multis locis prævalens. Fennia tantummodo ad Sammatti inventa (Mus. Helsingf.).

Var. nilssoni Malm (Göteb. Samh. Handl., 1850) : t. gracilis, cylindrica. albido-cornea, subhyalina, anfr. 5; long. 5, lat. $1^{5} / 6 \mathrm{~mm}$. - Suecia in Skåne (ad Ignaberga pr. Kristianstad).

Var. collina Drt. ap. Moq.-Tand. (Hist. Mal. Fr., 1855): t. par"va, ovato-oblonga, fuscovirescens $\mathrm{v}$. fuscorufescens, apertura piriformis, peristomate parum incrassato; long. $3-4$, lat. $1 \frac{1}{2}-2 \mathrm{~mm}$. - Suecia passim.

\section{Gen. Cæcilianella Bourg.}

T. gracilis, cylindracea, alba, hyalina, apertura 1/2 longitudinis totius subcequans, sursum longe et angustissime attenuata, margine tenui, columella basi tmuncata.

C. acicula Müll. (Verm. Hist. II, 1774, Buccinum). T. fusiformecylindracea; anfr. 7 , planulati, sutura perobliqua. Long. $5-6$, lat. $1^{1 / 4}-1^{1 / 2} \mathrm{~mm}$.

Hab. Srecic in Skåne (Lund), Småland (Jönköping), Öland (Högsrum). - Norv ad Kristiania (Aur. \& Arth. Krause 1880). - Dania in et circa Kjöbenhavn, ad Rude, Roeskilde et Holsteinborg, nec non (fide H. Lynge) in Jylland (ad Aarhuus et Viborg).

\section{Sectio 2. Diplotrema W.}

M. orificiis org. genit. externis discretis.

\section{Fam. Succinidr.}

T. imperforata, tenuis, oblongo-ovata, pellucida, spira acuminata, apertura magna, sape maxima (longitudinem fere totam testa occupans), verticalis, peristomate simplice, recto. 


\section{Gen. Succinea Drap.}

T. anfiactubus 3--4, ultimo maximo, convexo $v$. ventroso, apertura ovali.

1. Neritostoma Tilein. T. anfractu ultimo convexo v. ventroso, apertura late ovata, dimidiam testce longe superante. (Maxilla fuscobrunea, tridentata).

S. putris Lin. (Syst. Nat. X, 1758, Helix). T. ovalis, succinea, tenuis, spira late conica. acuta; anfr. convexiusculi, penultimus subtus convexinsculus, ultimus supra a sutura fere regulariter convexus, sutura levis, demum sepius forte profundeque descendens. Long. 15-17, lat. $7-9$, apert. l. 10-11, lat. $7 \mathrm{~mm}$.

Hab. Suecia ubique usque in Lule Lappmark (ad Qvickjock: H. Boheman). - Nore. usque in Ostfinmarken (ad Altenfjord sub $70^{\circ}$ lat. bor. invenit J. E. Z.). - Dania frequens. Fennia vulgaris usque ad Patsjoki in Enare Lappmark.

Forma gigantea Bourg. (Mon. Succ. fr., 1877), t. acute elongata, vix ventricosa, striata, sæpius rubra, apertura piriformis, sursum attentuata, basi rotundata; long. 19-21, lat. 11-12 mm. - Suec. in Öland (Treta). - Fennia ad Jynkkï (J. A. Sandman), mut. pallida in Åland, mut, rubra in Hogland (Mus. Helsingf.). - S. putris v. grandis Haz.

Var. limnoidea Pic. (Moll. Somme, 1840, f. Bourg. = clessisiniana Haz.) forma gotlandica $\mathrm{W}$. (Fauna Binnenconch. $\mathrm{V}$, 1885̃): t. longa, recta, acute conica, rugoso-striata, persolida et crassa, anfr. 4 , planati, apert. verticalis, ovalis; long. 25-27, lat. 11-12 mm. - Suecia in Gotland (ad Kopparviksdam et Etelhem). - Dania ad Bornholm (ad Stammershalle: Coll. Clev., et in Möens klint: H. L.). Femia in Aland, Hogland, Satakunta, Iläne (Mus. Helsing.f.).

Var. charpentieri Dum. \& Mort. (Cat. Moll. Sav., 1858): t. ovata, irregulariter rugulosa, spira brevi, late conica, anfr. : 3 (1. minimus, 2. brevis, convexus, ultimus magnus, ventrosus); long. 15, lat. 10, apert. long. 11, lat. $7 \mathrm{~mm}$. - Suecia in Blekinge (Romneby etc.), ad Borås et in Upland (ad Jürfra: V. L.).

Var. ferussina Moq.-Tand. (Hist. Moll. Fr., 185̃ō): t. parva, obesa, ovoidea, tenuis fragilisque, spira brevis, late conica, anfr. 3, superi minuti, obliqui, ultimus supra aperturam 
fortius convexus, apert. late piriformis; long. 9-11, lat. 51/2-6, ap. long. 6-7, lat. 4--5 mm. - Suecia in Fyrisån ad Upsala: P. T. C. - Fennia ad Itkoniemi in Kuopio: K. M. L.

Var. subglobosa Pascal (Cat. Moll. Haute-Loire, 1873): t. ventrosa, plicato-striata, tenuis, pallide flava, spira breris late conica, apert. magna, elliptico-rotundata, columella medio concava; long. 17-18, lat. 9, ap. long. 12, lat. $7 \mathrm{~mm}$. Suecia in Blekinge (Ronneby), Jemtland (ad Kall: E. N.) et ad Strömnäs pr. Piteå. - Dania in Möen (ad Lislund: $\mathrm{H}$. L.) - Fennia in Hogland et ad Jyväskylä (Mus. Helsingf.).

Forma drouetia Moq. Tand. (Hist. Moll. Fr., 1855), t. pallide flava, spira paullo longiore, anfr. minus convexis, apertura ovali. - Suecia in Skåne (ad Fogelsång prope Lund), Bohuslän (ad Qvistrum: P. T. C.), in Dalarne et in Gestrikland (ad Gefle). - Dania ad Ringstad.

Var. radiata C. G. W. apud W. (Fauna Binnenconch. Suppl., 1890): t. succinea, strigis transversis fuscis et albis alternantibus, spira pallide rosea, acuta, quam apertura semper duplo breviore, anfi: penultimus convexus, sutura antice lente profundeque descendens, plica columellaris alba; long: 14, lat. 8, ap. long. 10, lat. $6^{1 / 2} \mathrm{~mm}$. - Suecia in rupibus exterioribus maris baltice provinciæ Blekinge.

Var. trianfracta Da Costa (Brit. Conch., 1778, Turbo): t. late ovata, firma, levigata, cinereo-flavida, spira brevi, obtusa, anfi. convexi, sutura profunda, antice parum obliqua, apert. late ovata, columella arcuata, albocallosa; long. 15-16, lat. $9-10$, apert. long. 11, lat. $6 \mathrm{~mm}$. - Suecia in Blekinge. - Dania ad Rönne, Stampen, Wellingau etc., nec non in Bornholm.

Var. temporalis W. (Exposé crit., 1871): t. elongato-ovata, pertenuis, fragillima, striatula, pallide flavovirescens, intus margaritacea, anfr. 4, ultimus ad suturam planatus, sutura subcapillacea, antice subhorizontalis, brevius producta; long: 16-17, lat. 8-9, apert. long. 11-12 mm. - Suecia ad Ronneby (ad Herrstorpsjön).

Var. charpyi Baud. (Journ. Conch. XV, 1879): t. ventricosissima, spira brevi, conica, acuta, anfr. convexi, ultimus infra attenuatus, apert. elongato-ovata, $2 / 3$ longit. totius. Suecia in Gotland, Småland (ad Vernanäs), Dalarne et ad Stockholm (Bellevue).

S. parvula Pascal (Cat. Moll. Hte Loire, 1873). T. ventrosoovata, sat firma, spira perbrevi obtusiuscula; anfr. 3, con- 
vexi, primus tuberculo similis, sutura minus obliqua; apertura maxima, margine columellari curvato. Long. 8-11, lat. $6-7$, ap. long. $6^{1 / 2-}-7^{1 / 2}$, lat. $4-4^{1 / 2} \mathrm{~mm}$.

Hab. Suecia in Blekinge (Ronneby), Östergötland (Brunnby), Dalarne (Hedemora), Stockholm (Skärsätra in Lidingön: V. L.), Medelpad, Westerbotten et ad Torneå, semper in vicinitate proxima vel etiam in litoribus maris. - Fennia in palude Maljalampi in Kuopio (Mus. Helsingf.).

S. groenlandica Beck (Index Moll., 1837). T. elongata, sat firma, vix nitidula, pallide cornea, albido-strigata, spira scalaridea; anfr. 3, penultimus valde convexus; apertura ovalis, columella subrecta, margine exteriore arcuato. Long. S, lat. $5^{1 / 2}$, ap. long. 5 , lat. $3^{1 / 2} \mathrm{~mm}$.

Hab. Istandia. »S. Sp. grönlandica aff. Long. 11, lat. 7 mm., long: apert. 6, lat. $4^{1 / 2} \mathrm{~mm}$. - Dania, exemplar unicum laesum a Bierremark in ins. Lolland» (Mörch Syn. Moll. Dan.).

2. Amphibina Hartm. T. anfiactu ultimo convexiusculo, supra infraque angustato, apertura anguste ovatu, dimidiam teste longe superante (1Laxilla fuscoflaviria. medio dente prominulo).

S. pfeifferi Rossm. (Icon. I, 1835). T. obliqua, elongata, striata et striatula, spira brevi, conica; anfr. penultimus subtus extuspue ventrosus, superne plus minusve planatus, sutura demum minus obliqua; inter parietem et columellam angulus distinctus. Long. 12, lat. 6, ap. long. 8--9, lat. $5 \mathrm{~mm}$.

Hab. Suecia ubi forma typica Rossmässleri (Icon. f. 46) rarissime loco unico (in litore lacus Svinösjön in Dalarne) inventa est.

Var. elongata Hartm. ap. Sturm (Faumula Helv. VI, 1829): t. spira elongata, acuta; long. 15-16, lat. $7-8$, ap. long. 10, lat. $6 \mathrm{~mm}$. - Suecia a Skåne usque in Lappland, multis locis frequentior quam S. putris. - Norv. tota usque in Westfinmarken. - Dania tota vulgaris. - Fennia tota usque in Lapponiam.

Var. brevispirata Baud. (Journ. Conch., 1877): t. spira perbrevi, anfr. 3, celerrime accrescentes (1. minimus, 2. sat convexus, 3. maximus, elongatus, convexiusculus), apert. maxima, in- 
fra elongata; long. 9--11, lat. $5^{1 / 2}$, ap. long. $7-71 / 2$. lat. 4 mm. - Suecia ad Ronneby (in Herrstorpsjön). Göteboro (in Qvillebäcken), Jöuköping (in Lillsjön), Westergötland (Mösseberg), Upland (ad Upsala et in Lenna sjö). — Fennia in Åland ad Jomala, in amme Borgå (Mus. Helsingf.) et in Esbo-Löfö (K. M. L.).

Forma costellata mh., t. dense costnlata. - Suecia ad Stockholm (Nacka*, Carlberg: J. W.), in Södermanland (in lacu Sillen prope Charlottendal: A. d'A.) Dalarne (ad Hedemora) et Jemtland (ad Pilgrimsstad: E. N.).

Var. nilssoniuna Cless. (Malak. Blätt., 1579): t. ovata, solida, sat crassa, ochracea ad fuscorufa. spira brevis, acuta, anfr. $3--31 / 2$, perconvexi, sutura profunda, apert. rotundato-orata; long. 10-11, lat. 51/2-6, ap. long. $6^{1} / 2$, lat. $4 \frac{1}{2}$ mm. Snecia in Östergötland (in lacu Tåkern), ad Stockholm (Norrtull: P. de L.), Upland (Upsala), Medelpad. - Dania in Bormholm. - S. ochracea W. pr: p. olim.

Forma major long. 12-15, lat. $6-71 / 2$, ap. long. 7-9, lat. 5-6 mm. - Suecia in Gotland (Bunge).

Var. mediolanensis Villa (Cat. Moll. Lomb., 1844): t. elongatoovata, firma, tenue striatula, perobliqua, spira brevis, anfi. vix 3, conrexi, ultimus ad sinistrum et antice rentrosus, sutura profunda, antice subhorizontalis, columella concara; long. 8-9, lat. $4 \frac{1}{2}$, ap. long. 5-6, lat. $3^{1 / 2} \mathrm{~mm}$. - Suecia in Öland (ad Kohlstad prope Borgholm, sat frequens).

Var. diphya W. (Fauna Binnenconch. $\mathrm{T}, 1855$ ): t. tenmissima, perobliqua, ad sinistrum perconvexa, spira albida, anfr. ult. brumnescens, striatulus, a medio extus flavescens et forte rugoso-plicatus, basi longe attenuatus, apert. intus margaritacea, marginibus callo albo conjunctis; long. ad 12, lat. $6^{1} / 2$. ap. long. 9, lat. $5 \mathrm{~mm}$. - Suecia in Skåne (ad Sjöbo: J. W.) et ad. Stockholm (Norra Brumnsviken * prope Roslagstull: J. WW., ins. Tegelön: P. de L.).

Tar. rentricosa Pic. (Moll. Somme, 1840): t. elongato-ovalis. spira dimidiam apertura attingente, anfr. convexi. nltimus supra aperturam rentrosus, apertura superne acuta. infra dilatata et elongata, columella arcuatula. - Suecia in Lappland (Muoniovaara). - Femnia (Lapponia Rossica ad Ḱusräka: Mus. Helsingf.).

Tar. propinque Band. (Journ. Conch., 1877): t. elongata, anfr. nltimus validus, ventrosus, spira brevis, contorta, apertura magna, elongato-ovata, plus quam $2 / 3$ longit. totius; long. 12-22. lat. 7-12 mm. - Suecia ad Testerås. 
Var. elata Baud. (Journ. Conch., 1877): t. elongata, gracilis, angusta, obliqua, striatula, fuscoseracina, spira longa, acuta, forte contorta, sutura perobliqua, anfr. penultimus ralde, ultimus parum convexus, apert. ovalis, infra angustata; long. $10-15$, lat. 5-7, ap. long. 6-9, lat. $4-6 \mathrm{~mm}$. - Suecia in Öland (in stagno ad Petgärde: P. de L., et ad Tveta).

Var. contortula Baud. l. c.: t. parva, firma, elongato-ovata, dense striata et irregulariter plicata, spira longa, anfr. $3{ }_{1}^{1}$, sutura profunda; long. 8-9, lat. $4-41 / 2$, ap. long. 5, lat. 3 $-3 \frac{1}{2}$ mm. - Suecia in Skåne (Lund), Blekinge, Öland (Borgholm). - Norv. in Hole et Tromsö. - Dania ad Kjöbenhavn (in horto botanico, in fossis circa urbem et ad Rosenborg). - S. intermedia Bean.

Var. contorta W. (Exposé crit. 1871): t. oblonga, solida, striata, spira elongata, acuminata, apice rosea, anfi. $3 \frac{1}{2}-4$. valde contorti, supremi conrexi, ultimus elongatus, juxta suturam forte descendentem planulatus; long. 12-16, lat. 6-7, ap. long. 7-8, lat. $5 \mathrm{~mm}$. - Suecia in Gotland (Träskhedarne* in par. Bunge et Fleninge) nec non in Westerbotten (Piteå). - Norv, in Stensfjorden. - S. ochracea IV. pr. $\mathrm{p}$ olim.

Var. gracilis Ald. (Mag. Zool. a. Bot., 1837): t. membranacea, paliide flava, subcylindrica, anfractus convexi, spira obtusa; long. 15. lat. $61 / 2 \mathrm{~mm}$. - Dania, Sjælland (in hortu ad Dronninggaard).

Var. subelegans W. (Nachr.-blatt Mal. Ges., 1894): t. perangusta, elongata, tenue striata, pallide succinea, anfr. 3, ult. basi longe attenuatus, longus, angustus, marginibus subparallelis, penult. convexus, sursum conico-attenuatus, sutura inferne peroblique descendens, apert. Ionga, sursum attenmata, infra dilatata, columella subrecte in marginem columellarem transiens; long. 7, lat. 3 mm., apert. long. $51 / 2$ mm. - Dania in Fyen (ad Odense: H. L.). - Fennia ad Sysmä (Mus. Helsingf.).

S. Ienta $\mathbb{T}^{\mathrm{T}}$. (Öfvers. K. Vet. Ak. Förh., 1881). T. parum oblicqua, subpiriformis, tenuissima, flexilis, subplicata et tenue striata, spira brevissima, contorta; anfr. penultimus parvus, convexus, ultimus sursum planatus, sutura parum obliqua; apertura obliqua, infra dilatata, basi retusa. Long. ó, lat. $4^{1} / 2$, ap. long. $6^{1 / 2}$, lat. $4 \mathrm{~mm}$.

Hab. Suecia in Skåne (Kristianstad) et Blekinge (Ronneby et Nättraby). 
Forma de lavali, testa long. 14. lat. 8, ap. long. 9, lat. $7 \mathrm{~mm}$. - Suecia in Östergötland (Eksund *: P. de L.).

S. altaica Mart. (Sitz. ber. Ges. Berlin, 1871) var. norvegica W. (Fauna Binnenconch. V, 1885). T. elongato-ovata, rugosostriata, alboflavida, spira elongata, valde contorta, obtusa; anfr. $3^{1 / 2}-4$, convexi, sutura obliqua, profunda; apert. superne non angustata, altitudine spiræ, columella curvata. Long. 12, lat. 7 , ap. long. $6-6^{1} / 2$, lat. $4 \mathrm{~mm}$.

Hab. Norvegia in Lofoten (ins. Renö). Islandia (Feddersen). - S. pf. v. contortula in Exk. Fauna 1884.

3. Lucena Oken. T. anfractu ultimo convexo, apertura late ovali v. rotundata, vix spira breviore. (Maxilla pallide fulva, unidentata).

S. oblonga Drap. (Tabl. Moll., 1801). T. gracilis, tenuis, at firma, striatula, virescenti-lutea, spira sat alta; anfr. 4, ultimus parum convexus, sutura obliqua, profunda; apert. anguste ovata, $1 / 2$ testre long. æquans. Long. 7, lat. $3^{1 / 2}-4$, lat. $3-4 \mathrm{~mm}$.

Ha乙. Suecia in Småland (Jönköping), Östergötland (Omberg), Bohuslän, Stockholm (Humlegården, Nacka), Westmanland (in Björnö), Helsingland (Arbrå: E. C.) et Jemtland (Östersund et Offerdal: E. C. \& E. N.). - Norv. in Malmöen, Lindöen, Hole, Nordenhoug. - Dania fere tota passim.

Forma impura Hartm. ap. Sturm (Faunula Helv., 1829), t. parvula, ovato-oblonga, leviter striata, limo sæpissime obducta, spira brevis, anfr. 3, subtorti, apert. rotundato-ovalis, long. 6-7, lat. 4, ap. long. $3 \frac{1}{2}-4$, lat. $3 \mathrm{~mm}$. - Suecia passim cum typo (et ad Stora Råby prope Lund? = S. arenaria Nalm). - Dania in Sjælland (Söndermarken). - Pullus?

*S. agonostoma Kstr. (Bamberg. Ber. II). T. elongato-ovata, gracilis, solida, crassiuscula, spira elongata, acuta, forte contorta; apert. angusta, marginibus callo albo conjunctis, exteriore intus labiato. Long. 7, lat. $3-3^{1} / 2$, ap. long. $3^{1 / 2}$ -4, Iat. $2^{1} / \mathrm{s} \mathrm{mm}$.

Hab. Suecia in Blekinge (Sölvesborg inter ruinas arcis et mare: C. Brovallius). - Norv. ad Hole.

+S. Iutetiana Mab. (Hist. mal. bas. Paris, 1870). T. late ovata, 
tenuis et fragilis, subplicato-striata, virescens, spira breve lateque conica; anfr. 3-31/2, rotundato-convexi, ultimus obliquus, valde ventricosus; apert. rotundato-ovalis, ${ }^{2} / 3$ long. testie non attingens, columella curvata, celeriter superne intrans. Long. 7 , lat. 4, ap. long. 4, lat. $3 \mathrm{~mm}$.

Hab. Dania ad Kjöbenhavn (in hortu poss. Olsson ad Österbro). - S. crosseana W. in Exk. Fauna 1884.

S. borealis n. sp. T. minuta, angusta, oblongo-conica, irregulariter striata v. striatula, alba v. albido-succinea, spira producta valde contorta, acuminato-conica; anfr. $1-41 / 2$ convexi, extus infra ad suturam profunde immersam obliquam constricti, ult. elongatus, convexiusculus; apert. longitudine spiræ, irregulariter-oblonga, basi rotundata et recedens, margine exteriore ab insertione forte curvato, columella strictiuscula, cum pariete angulum formante. Long. 6, lat. $2^{3} / 4 \mathrm{~mm}$.

Hab. Suecia in Jemtland ad Östersund (E. N.).

S. arenaria Bouch. (Cat. Moll. Pas-du-Cal., 1838). T. elongatoovata, sat crassa et firma, rufosuccinea, rugoso-striata, spira elongata, forte contorta, late conica; anfr. $3^{1 / 2}-4$, perconvexi; ultimus ventricosus, sutura profunda, antice subhorizontalis; apert. rotundato-ovalis, intus polita, longitudine spirse requalis; margine exter. forte curvato, m. colum. superne reflexiusculo. Long. 7-8, lat. 3-4, ap. long. 32/3-4, lat. $2^{1 / 2}-3 \mathrm{~mm}$.

Hab. Suecia in Gotland (Träskhedarne par. Bunge, Bungenäs in Ruthe, Snäckgärdet pr. Visby, Ejketräsk etc.), Öland ad Borgholm (in Borga hage) et in Jemtland (ad Östersund: E. N.) - Norv. ad Jerken, in Langö, in Helgoöen in lacu Mjösen. Dania in Lolland (Bjerremark) et ad Præstö.

Forma impura, t. limo obducta, long. 5--6, lat. 3-31/2, ap. long. $2 \frac{1}{2}-3$, lat. 2-21/2 mm. - Cum typo. - Pullus?

Subordo 2. Hygrophila Fér.

1I. pulmonata, tentaculis :2, contractitibus, basi oculiferis. 


\section{Fam. Anriculida.}

11. tervestria, tentaculis brevibus cylindraceis. T. ovatoollonga, apertura dentata.

\section{Gen. Carychium Müll.}

T. minima, alba, apertura obliqua, longior quam lata.

C. minimum Müll. (Verm. Hist. II, 1774). T. nitida; anfr. 5$5^{1} / 2$, convexi, ultimus maximus; apert. lamella infra medium parietis et denticulo in columella, peristomate reflexo, margine exteriore forte labiato, labio supra medium dentato. Long. 2, lat. $1 \mathrm{~mm}$.

Hab. Suecia sat vulgaris a Skåne saltem usque ad Östersund in Jemtland (unde miserunt cl. E. C. \& E. N.). - Nort: meridion. et media. - Dania vulgaris. - Femia in Åland, Pargas, in par. Mäntsälï, ad Borgå, Kuopio, in par. Kiiminki et Paltamo (A. J. Mela).

\section{Fam. Limnaida.}

M. aquatilia, tentaculis planis, triangularibus v. subulato-setaceis. T. forma pervariabitis, apertura semper. edentata.

Subfam. Limnaina.

$T$. dextrorsa, oblonga, ovata $v$. subglobosa. Animal tentaculis planis, triangularibus.

\section{Gen. Amphipeplea Nilss.}

T. pallio animalis ommino involuta, tenervina, fragitlima, nitidissima.

A. glutinosa Müll. (Verm. Hist. II, 1774, Buccinum). T. subglobosa, subplicato-striata, pallide lutescens, tenuissime spiraliter lineata; anfr. 3, ultimus ampullaceus totam fere testam efficit; apert. maxima. Long. 10, lat. 8, ap. long. 9, lat. $7 \mathrm{~mm}$. (18-13-16-13 mm.).

Hab. Suecia in Skåne multis locis, Blekinge (Romneby). Halland (Skottarp), Westergötland (Göteborg et in amne Mölndal), Nerike (in lacubus Tisaren et Sottern), Södermanland (in Öknasjön), Stockholm (Wermdö), Lpland (Upsala), Westmanland 
(in Mälaren ad Wiksäng et in lacu ad Axholm), Wermland (in amne ad Forsevik), Dalarne (in Sarbosjön par. Gustaf). - Dania in Sjælland multis locis nec non in Falster (Stubbekjöbing: H. L.). - Femia late destributa (ut ad Lojo, Säïksmäki, Jyväskylä, Kajana, Hyrynsalmi, Kianto, Viborg, Kallavesi, Rantasalmi, Laitila, Varsuga in Lapp. ross. \&e.).

Gen. Limnæa (Brug.) Rang.

T. pallio animalis incluso (ut apud genera serpentia).

1. Lymnus Montf. T. elongato-ovata v. turita, tenuis, at firma, clathrata, spira elongata, acuta, srepe aperturce longitudine cequalis, anfractus 6-S, ultimus magmus, inflatus, apert. intus pallirla, albida.

L. stagnalis Lin. (Syst. Nat. X, 1758, Helix). T. sat solida, pallide cornea, nitidula, sub lente tenuissime densissimeque spiraliter lineata, spira turrita, obscuriore; anfr. 7 , ultimus elongato-ventricosus, superne angulatus; apertura ovata. Long. 50-60, lat. 26-29, ap. long. 26-34.

Hab. Suecia per maximam partem regni saltem usque in Lule Lappmark $\left(67^{\circ}-68^{\circ}\right.$ lat. bor.), tamen in legionibus certis (ut prov. Blekinge fere tota, circa Göteborg etc.) omnino desideratur. - Norv. Lota usque in Ostfinmarken (Pasvig). - Dania tota vulgaris. - Fennia tota, limitem Lapponix summum attingens.

Var. subulata W. (Exposé crit., 1871): t. gracilis, elongatoconica, spira subulata, quam apertura sæpe multo longiore, anfr. omnes 8-9 elongati, parum convexi, apertura oblongo-acuta; long. 40-50, lat. 14-19, ap. long. 18-20, lat. 9-10 mm. - Suecia in Skåne (Kristianstad), Östergötland (in Skogssjön), circa Stockholm (in Ältsjön et testa alba in Flatsjön: Hj. W.) et in Upland (Upsala). - Dania ad Kjöbenhavn, Sorö et Hörby nec non in Bornholm (in Store Geddesö: H. L.). - Fennia ad Impilahti et Luhanko (Mus. Helsingf.). - L. subula Parr. in se., L. subulata Dumk. ap. Küst. (1862) forma obseura et L. raphidia Bourg. (1860) e figura forma abnormis scalaridea est!

Tar, producta Colb. (Ann. Soc. Mal. Belg., 1859): t. solida, spira quam apertura longiore, acuminata, anfi. 9, lentis- 
sime accrescentes, planulati, ultimus panllo ventricosus et angulatus, apertura ampla, superne ei extns obtuse angulata, margine exteriore fere verticalis; long. 65, lat. 29, ap. long. 51, lat. $17 \mathrm{~mm}$. - Snecia passim. - Fennia ad Luhanko et in lacu Kolmisoppi ad Kuopio (K. M. L.).

Var. sophronic W. (Nachr. bl. Mal. Ges., 1893): t. cormeo-lutescens, concolor, forte malleata, spira longe et acute attenuata, apert. retrorsum obliqua, peristomate plus minusve reflexo; long. 45, lat. 20, ap. long. $20 \mathrm{~mm}$. - Suecia in Östergötland (in lacu Skogssjön*: Dr. H. Nerén). Testa forma omnino cum figura 175 varietatis ad Budapest Hungaria lecte in Iconographia Kobelti congrua.

Var. westerlundi Loc. (Conchyl. Fr., 1893): t. sat temuis. flavescens, spira elongato-conica, acuta, anfr. 7-8, lente regulariter accrescentes, superi planulati, nlt. plerumque convexus, supra planatus, non angulatus, apert. quam spira brevior, elongato-ovata; long. 50, lat. 18-20, ap. long. 23-25, lat. 10-12 mm. -- Passim, in Suecia usque ad Randijaur in Lule Lappmark. - Dania in Sjælland (inter Frederiksberg et Lilleröd: H. L.). - Femnia in Ảland (in palnde ad Torpby: Mus. Helsingt:). - L. st. var. vulgaris W., nec Jeffr.

Forma neréni WV. (Nachr. bl. Mal. Ges., 189f): t. cornea, striatula, strigis nonnullis eburneis ornata, apert. intus late eburneo-labiata, margine exteriore supra medium exciso, infra medium forte areuatim producto; long. 46, lat. 20, ap. long. 25, lat. infra med. $15 \mathrm{~mm}$. - Suecia prope ad Skeninge* frequens.

Var. colpodia Bourg. (Specil. Malac., 1862): t. cornea, striatula et sæpe ad suturam longitudinaliter transverseque sulcis tessellato-concavis, spira elongata, acuminato-conica, anfr. parum convexi, ad suturam marginatam parum obliquam planulati, apert. oblonga, acuta; long. 50, lat. $20 \mathrm{~mm}$., ap. long. 30, lat. $15 \mathrm{~mm}$. - Dania.

Var. yotlandica W. (Nachr. bl. Mal. Ges., 1891): t. crassissima, albida, striatula, anfi: 7, ultimus medio planatus, antice duplicatus, lamina interiore producta, margine supra medium late concavo, infir medium forte producto, peristomate recto, margine colum. in laminam latam perforationem subtegentem reflexo, marginibus callo lato tenui junctis. Occurrit sub formis duabus: 1. diffusa, spira productiole, acuta, tenui, apertura basi dilatata, margine columellari retrorsum arcuato, t. long. 36, lat. 20, ap. long. 23, lat. inferne $15 \mathrm{~mm}$. - 2. retusa, spira breviore, basi lata, 
superne forte acuminata, apert. majore, latiore, basi angustata, margine columellari recto, verticali, anfractu ultimo ventroso, t. long. 39, lat. 25 , ap. long. 25 , lat. $15 \mathrm{~mm}$. - Suecia in Gotland: (Coll. Cleveana).

Var. elegans Leach. ap. Turton (Brit. Moll., 1831): t. tenuis, flavescens, spira subulato-conica, strigis variciformibus parcis, sutura alba, apert. margine exteriore incrassato, sæpe pallide aurantiaco; long. $29-32$, lat. $12-14$, ap. long. $16-18$ mm. - Suecia in Skåne (Lund Hjelmslund in Rönneå et ad pagum Öja). -- Dania ad Kjöbenhavn. - Fennia ad pagum Västiniemi (Nilsiä) in Savolax (Mus. Helsingf.).

Var. roseolabiata Wolf ap. Sturm (Fauna VI. I., 1803): t. tenuis, brunnea, sæpe extus nigricans, intus lactea, margine exteriore aperturæ intus roseolabiata, spira conica, mediocris; long. 47-50, lat. 20-22 mm. -- Suecia in Skåne (Lund, Malmö \&c.), Upland (Upsala). - Dania in insulis late distributa, in Jylland ad Aarhuus.

Tar. tor'sa IV. (Exposé crit., 1871): t. magna, spira longa, antr. superioribus convexis, sutura profunda disjunctis, ultimo rentroso, superne angulato. - Suecia in Ostergötland (A. T. G.) et in Dalarne (Säter: $)$.

Var. turgida Menke (Syn. Moll., 1830): t. subovata, obesa, subrudis, spira brevi, acute conica, anfr. ultimus superne obtuse angulatus, subtruncatus, apert. quadrangularis, columella profunda sinuata; long. 40, lat. 26, ap. long. 23, lat. 12-13 mm. - Suecia passim (precipue in Skane et Östergötland). - Dania passim. - Fennia ad Jynkkä pr. Kuopio (Mus. Helsingf.).

Vax. borealis Bourg. (Specil. Mal,, 1862): t. ovalis, nitida, distincte spiraliter lineata, tenuis et junior fragilis, brumea, spira late conica, sensim acuminata, $1 / 2 \ldots 2 / 3$ longitudinis aperturx, anfr. 6- $71 / 2$, ult. ventrosus, superne rotundatus, sutura alba; long. 43, lat. 23, ap. long. 25, lat. $13 \mathrm{~mm}$. (41 $-23-20 ; 50-27-31 \mathrm{~mm}$ ). - Suecia in Gotland (Burträsk et Storsäf par. Mistermyr), Dalarne (Sätex), Jemtland (Lockne et Alfensjön), Lule Lappmark (Qvickjock) et certissime in multis locis adjacentibus. - Fennia vulgaris.

Vax. lacustris Stud, ap. Cox (Trav., 1789, Buccinum): t. solida, sæpius malleata v, clathrata, spira perbrevis, celeriter ab anfractu nltimo ventroso atteuuata, sæpe vix $1 / 3$ longit. aperturæ attingens, anfr. ult. superue sæpius subtransversus, long. 28-30. lat. 20, ap. long. 22, spira long. $6-7 \mathrm{~mm}$. (39-30-29-13 mm). - Suecia in lacubus in Skåne, Óstergötland, Nerike, Öland et Gotland. - Dania multis locis 
(ut ad Birkeröd, Juelsborg, Juulssö pr. Silkesborg, Höjby, Vibol'g).

Var. nordmanni IV. Nachr. blatt Mal. Ges., 1894): t. non limata, rotundato-ovata, irregulariter striata, cornea, tenuis at solida, spira brevissima, late conica, apice breviter acuminata, anfr. 5, ult. maximam testie partem oceupans, ventrosus, sutura usque ad apicem horizontalis, apert. late ovata, margine exteriore ab insertione forte arcuato, columellari curvato, pariete brevi, convexo; long. 25, lat. 20. ap. long. 20, spira long. $7 \mathrm{~mm}$. - Fennia ad Walamo* (A. v. Nordmann) et ad Pyhäjärvi (Mnus. Helsingf.).

Var. melia Hartm. 184 (Kob. Iconogr. f. 1235): t. firma, cornea v. rufescens, lrvigata, spira quam apertura brevior, conico-acuminata, anfr. ult. sat ventrosus, non angulatus, apert. infra rotundatus, margine exter. ab insertione regulariter arcuatulo-descendente, columella incrassata, forte contorta; long. 35, lat. 20, ap. long. 25, spira long. $10 \mathrm{~mm}$. - Suecia in Skåne (Ringsjön). Certe etiam in aliis locis. Bene observandum est hane formam in lacu Ringsjön eodem loco ac et promiscue cum forma omnino contraria, sc. maxime gracillima var. raphidiæ, occurrere.

Var. alata W. (Exposé crit., 1871): t. magna, flavescens v. pallide cornea, spira brevis, acuta, anfr. ult. ventricosoampliatus, superne obtuse angulatus, apert. amplissima, intus splendida, obscurior, peristoma auriculatim dilatatum, margine exter. superne horizontali, columellari infra extenso; long: 5̃5, lat. 29-33, ap. long. 33-35, lat. $23 \mathrm{~mm}$. - Suecia in Småland (in lacu Möen * ad Degla). - Fennia ad Harjula pr. Kuopio (K. M. L.).

Var. bottnica Cl. (Malak. Blätt., 1878): t. late rimata v. umbilicata, tenuis at firma, striata, sæpius lineis spiralibus elevatis, cinereo-flavescens, spira angusta, subscalaris, anfr. ult. subventrosus, supra sat transversalis, apert. elongata, sat angusta, margine exter, medio substricto, columellari rectiusculo, superne late reflexo; long. 18-23, lat. 10-13, ap. long. 9-13, lat. $\check{-}-\mathrm{S} \mathrm{mm}$. - Suecia et Fennia ad et prope litora Sinus Bottniei.

Var. baltica Lindstr. (Gotl. nutida moll., 1868): t. rimata, tenuis at firma, ovata, acuta, pallide fulvo-brumnea, anfr. 5 -6 , convexiusculi, ult. ventrosus, apert. magua, margine exter. forte arcuato, columellari infra arcuato, superue late reflexo; long. 22-24, lat. 12 , ap. long. 15-16, lat. 8 mm. (long. 30 , ap. 16, sp. 16; $30-17$ v. 20,-10 $\mathrm{mm}$ ). -- 
Acta Societatis pro Fauna et Flora Fennica, XIII, n:o 7.

Snecia ad litora maris baltici saltem usque ad Stockholm (ubi frequens et multiformis in Wermdö).

Var. livonica Kob. (Icon. N. F. f. 186-195, 1884): t. non v. vix rimata, solida, pallide cornea, regulariter striata, interdum subcostulata, raro malleata, spira elata, gracilis, anfr. 7, penult. tumidulus ut nltimus leviter humerosus, apert. longe ovatus, infira retusa, plica columellaris sæpe obliterata; long. 24-25, lat. 12-1', ap. loug. $15 \mathrm{~mm}$. - Fennia ad Esbo-Löfö. (Mus. Helsingf.). Vix dubito quin var. $\gamma$. Limnære stagnalis in Finlands Mollusker» p. 52 t. 3 f. $11 \mathrm{c}$ (de qua dicitur: ubique ad lapides totius litoris Fennir usque in teniis exteriolibus affixa) huc referenda sit.

Obs. Melius forte sit L. bottnicam et livonicam formarum loco var. balticre habere.

2. Gulnarra Leach. T. subovata, tenuis, spira brevis c. brevissima, apertura magna, scepius multo longior quam spira, anfr: 4-5, ultimus maximus, scepe inflato-venticosus, apert. intus pallida, non $v$. albolabiata, margine exteriore rerticali.

L. auricularia Lin. (Syst. Nat. X, 1758, Helix). T'. latiuscule rimata (sed rima sxpissime parte superiore reflexo et depresso marginis colum. plus minus obtecta), rotundato-ovata, spira brevi, angusta, conica, acutissima; anfr. ultimus inflatus, dilatatus, postice superne convexus, angustatus; sutura levis; apert. supra infraque rotundata et subxque lata, pariete brevi, sinu colum. profundo, margine super. horizontali v. breviss. ascendente, exteriore arcuato, subrecto. Long. 25, lat. 20, ap. long. 20, lat. $15 \mathrm{~mm}$.

Hab. Suecia late distributa, vulgaris in Skåne, Westergötland, Östergötland et.circa Stockholm, sparsim in Nerike (in Hjelmaren et Tisaren), rarius in Blekinge (in Långasjön pr. Ronneby et Emmahultsjön), Småland (in lacu Noen ad Degla), Wermland (ad Carlstad), Dalarne (in Åmungen par. Husby), Westmanland (ad Westerås), Upland (ad Upsala). In insulis (iotland et Öland non occurrit. - Norv. raro (Kristiania, Fure in Tyrifjord, Tveterelven in Transby). - Dania fere tota vulgaris. - Fennia meridionalis, nec non in ultima boreali parte ad Mare glaciale (ad Veskonine et Lueschjaure formas magnas invenit clariss. Ch. Rabot). 
Var. ventricosa Hartm. (Gasterop. d. Schweiz, 1811): t. rimata, ampullacea, tenuissima, fragillima, subhyalina, sublævigata. spira (anfr. 3 superi) minuta, prominula, mucronata, apert. ovata, columella curvata, sinus colum. sat profundus, peristoma rectum, margine exteriore ab insertione forte arcuato. - Suecia, ex. gr. in lacu inter Järfva et Sundbyberg (J. W.) et ad Stora Wärtan (Hj. W.) prope Stockholm. - Fennia in Ladoga et ad Laitila, Satakunta (Mus. Helsingf.).

Var. concilians W. (Fauna Binnenconch. T, 1855): t. non rimata, tenuis at firma, tumida, sordide virescenti-cinerea, striatula, spira conica crassa, celeriter acuminata, acuta, anfr. ult. maximus, inflatus, superne transversalis, apert. supra transversalis, intus corneo-flavida, nitidissima, mar. gine superiore horizontali $\mathrm{v}$. ascendente, columella prominente contorta, sinu sat valido, plica lata tota adpressa; long. ad 40, lat. 30, ap. long. 30, lat. $20 \mathrm{~mm}$. - Suecia in Nerike (in lacu Tisaren). Kob. lcon. N. F. f. 184-185.

Var. conalis Villa ap. Dupuy (Hist. Moll. Fr., 185́1): t. oblique orata, tumida, spira brevissima, late conica, acuta, axis longitudinalis perobliqua. apert. magna, superne angustata. obtuse angulata, infra valde dilatata, late rotundata, margine superiore brevi horizontali, exteriore oblique descendente; long. 20--30, lat. 15-20, ap. long. 18-23, lat. 13$15 \mathrm{~mm}$. - Suecia in Östergötland (lacus Tåkern). - Dania ad Viborg.

Var. elongata Kob. (Icon. f. 1518), aff.: t. oblonga, spira brevi, acuta, anfr. penultimus convexus, ult. subtus inflato-convexus, extus convexus, basin versus longe attenuatus, a pert. margine exteriore leviter regulariterque curvato, columella longa, reflexa, margine colum. arcuato; long. 20, lat. 11, ap. Iong. 15, lat. $10 \mathrm{~mm}$. - Suecia ad Stockholm in Brunnsviken (Hj. IV.).

*L. ampla Hartm. (Gasterop. d. Schweiz, 1844). T. subglobosa, spira brevissima, subimmersa, subulata; apertura amplissima, subcircularis, intus fulvida, nitidissima, margine superiore ascendente, sape valde super apicem producto, exteriore late patulo v. reflexo, sinu columellari et plica fere obsoletis. Long. 20, lat. 18-20, ap. long. $20-25 \mathrm{~mm}$.

Hab. Srecia ad Stockholm (Carlberg et Brunnsviken: Hj. W.) et Westervik, in Roslagen (in lacu Erkan) et in Östergötland (in lacub. Tåkern, Ensjön \&e.: J. W.) - Dania in Sjicl- 
land (Tiustrupsö pr. Skjerkör, Gyrstingesö pr. Ringstad, Bavelsesö). - Fennia in lacu Lojo et in Ylijärvi ad Uskela, Munrila (Nus. Helsingf.).

Forma pemile Mörch (Syn. Moll. Dan., 18644), t. semipollicaris, suborbicularis, planulata, limo terrugineo obducta, labro reflexo. . - Dania in Bavelsesö.

Var. plicata mh.: t. parva, rufescens, anfi. 4, apice prominulo, columella arcuata, plica prominente; long. 12, lat. 10, ap. lg. $10 \mathrm{~mm}$. - Strecia in Skåne (ad Malmö) et ad Stockholm (in Brunnsviken *: V. L.). Kob. Iconl. f. 12.t8.

Var. monnardi Hartm. l. c.: t. tenuis, spira immersa, margine aperturae superiore multo humilior, a fronte non conspicua, apert. amplissima, margine superiore valde ascendente, columella et pariete brevissimo fere in linea recta obliqua, sinu obsoleto, margine exteriore valde arcuato, plane dilatato; long. et lat. $25 \mathrm{~mm}$. - Suecia in Skåne passim, in Östergötland (in lacu Tåkem).

L. mucronata Held (Isis, 1836) var. alpestris Cl. (Exk. Fauna, 1876). T. non v. anguste rimata, subcirculari-ovata, tonuissima at firma, cornea, spira brevi, basi lata, celerrime acuminata, mucronata; anfr. 5, convexi, sutura impressa, antice horizontali, ultimus maximus, antice convexus, postice globoso-tumidus, subtus forte attenuatus, levissime declivis; apert. magna, margine exteriore et columellari longo ac reflexo regulariter areuatis, sinu distincto. Long. 20, lat. 12, ap. $\mathrm{lg} .14-15 \mathrm{~mm}$.

Hab. Femia in Kuopio (in lacu Kallavesi: K. M. L.). Specimina omnia, quæ a loco citato vidi, tandem aperturam monstrosam habuerunt.

L. lagotis Schr. (Fauna Boica III., 1803, Buccinum). T. vix rimata, ovata, tenuis, spira elongata, acuminata, forte contorta, sutura canaliculata, antice breviter ascendente; anfr. ultimus sat ventricosus, infra elongatus et dilatatus; apert. superne rotundata, margine breviter horizontali, angustata, $2 / 3-v_{i x} 3 / 4$ longitudinis testæ. Long. 17-20, lat. 10-12, ap. $\lg .12-14$, lat. $8 \mathrm{~mm}$.

$H a b$. Succiu sparsim a Skåne usque in Lule Lappmark (ad Qvickjock). - Norv. rarius (Kristiania, Brevik, Asker, Rormo 
in Oier, Valders etc. saltem usque in Nordlanden ut ad Akershuus: Ch. R.). Forte huc L. mucronata B. Esm. e Westfinmarken. - Dania »tota passim.» (O. Mörch in Syn. Moll. Dan. canalem ad Charlottenlund Söndremarken, stagnum ad Lundehuus et »multa alia loca» citavit; dicam vero me nullum specimen typicum in collectione formarum hujus generis e Dania ditissima amici H. Lynge detegere potuisse). -- Fennia in palude Valkeinen ad Knopio, ad Mullinkoski prope Fredrikshamn, ad Kytö in Esbo et Schlüsselburg (Mus. Helsingf.).

Var. diploa mh.: t. plus minusve aperte rimata, tenuis, sat firma, flavido-cornea, nitidula, spira elongata, celeriter acuta, anfi. 5, convexi, ultimus ventricosus, medio planatus, infra brevissime elongatus, apert. oblongo-ovata, basi rotundata, margine colum. reflexo, substricto, exteriore leviter curvato, medio rectiusculo; long. 24, lat. 14-15, ap. lg. 15-16 $\mathrm{mm}$. - Suecia in Skine (ad Stehag : Hj. W.) et Gotland (ad Kopparvik: V. L.). Kob. 1. Nachtr. Fauna Nass. Moll. 1886 t. 9. f. 4.

Var. alata Sporleder (Kob. Icon. f. 1241): t. late ovalis, apert. ampla, peristomate auriculatim dilatato, late limbato, subreflexo, margine superiore horizontali; long. 18-20, lat. 13 -14, ap. lg. $15 \mathrm{~mm}$. -- Suecia in Östergötland (Borghamn).

Var. anderssoni Cl. (Malak. Blätt., 1878): t. tenuissima, fragilis, striatula, spira mediocri, acuta, anfr. ult. inflatus, superne forte dilatatus et transversus, apert. magna, margine exteriore circulari, plica columellari lata; long. 14, lat. 11, ap. lg. 10, lat. $7 \mathrm{~mm}$. - Suecia a prov. Nedelpad septemtrionem versus ad litora maris. - Fennia ad Getha Alandiæ, ad Löfö in Esbo, in Korpo (Pensar), in litoribus marinis (Mus. Helsingf.).

Var. margaritacea W. (Sver. Moll., 1865): t. tenuissima et fragillima, pellucida, flavescenti-cornea v. succinea, spira unnc brevissima, nunc paullo producta, supra fortissime attenuata, acuta, anfr. ultinus ventrosus, apert. magna, intus plus minusve margaritaceo-nitens, margine exteriore ab insertione forte arcuato, columellari forte curvato, pariete convexo, infra extus columellam acutam, contortam depresso; long. 1621, lat. 11-16, ap. lg. 11-16, lat. 9-10 mm. - Suecia in Skine (Säbyholm), Blekinge (in Li̊ngasjö* ad Ronneby), Smi̊land (ad Hofryd), Östergötland (in Kilarpsjön ad Helgeslätt).

Var. amicula W. (Fauna Suecix, 1873): t. perforata, ovata, 
striata, anfr. 5, ultimus ventricosus, spira exserta, arcte contorta, acuta, apert. fere semicircularis, margine exteruo regulariter arcuato, margine colum. longo, subrecto, pariete brevi, simu profundo; long. 19-21, lat. 12-14, ap. lg: 15-16, lat. 9-10 $\mathrm{mm}$. - Suecia in Skåne (ad Örtofta ${ }^{*}$ et in Höjeă frequens). -- Dania in Bornholm.

H. prisca II. (Öfvers. K. Vet. Ak. Förh., 1881). T. sat elongata; anfr. 4, ultimus a dorso visus valde obliquus, extus perangustatus, al rotundatus, aperturam versus forte et priesertim infra dilatatus, convexo-tumidus; spira valde contorta, forte attenuata, a tergo visa perangusta; anfr. penultimus longus, sursum attenuatus, basi inflexus, ultimus obliquus; apert. magna, margine exteriore forte arcuato, columellari longo, subrecto. Long. 7, lat. vix 5, ap. lg. $4^{1} / 2 \mathrm{~mm}$.

Hab. Succia in Skåne (ad Stenstorp, in paludibus turfosis, sulbfossilis).

HL. ampulla Küst. (Chemn. Conch. Cab., 1852) forma lavis m.: t. aperte lateque rimato-perforata, ampullaceo-ovata, tenuis, fragilis, olivaceo-flavida, levigata (ap. typum »regulariter striata, striis superioribus cariniformibus»); anfr. convexi, tres superi spiram parvam subobtusam formantes, ultimus validus, superne tumido-ventrosus, ad suturam impressam planatus, infra medium forte angustatus; apert. ovata, superne perobtusa, margine exteriore ab insertione (ad v. infra medium anfr. pen.) forte arcuato, stricto, columellari curvato, supra reflexiusculo, sinu col. obsoleto. Long. 24, lat. $18-20$, ap. $\lg .18-22$, lat. $13-15 \mathrm{~mm}$.

Hab. Suecia in Jemtland (Storsjön: E. N.).

L. ovata Drap. (Hist. Moll., 1805). 'T. rimata, ovata, tenuis, spira breve lateque conica, obtusa v. obtusiuscula, sutura conspicua, antice horizontalis; anfr. ult. convexus, elongatus; apert. elongato-ovata, superne acuminata, margine exteriore ab insertione descendente. long. 20, lat. 1', ap. lg. 15 , lat. $8 \mathrm{~mm}$.

Hab. Suecia tota usque ad summum septemtrionem sub una alterave forma, typica major ubique rarior, forma minor 
vulgaris. - Norv, tota usque ad $70^{\circ}$ lat. bor. - Dania et Fennia ubique.

Var. intermedia Mich. (Compl., 1831): t. elongato-fusiformis, tenuis at firma, corneo-flavescens, spira elongata, sat gracilis, acuta, anfi: convexi, ultimus infra sensim attenuatus sutura antice obliqua, apert. oblongo-ovata, marg. exter. leviter curvato, columella curvatula, reflexa, adpressa; long. 20, lat. 10-12, ap. lg. $13 \mathrm{~mm}$. - Suecia in Upland (V. L.). - Dania ad Besserup (Steenbuch).

Var. surcula mh.: t. rimata, elongato ovata, fusco-cornea, nitida, firma, dense striata, spira elongata, late conica, obtusiuscula, anfr. tres ultimi fortissime accrescentes, convexi, penultimus magnus, ventrosulus, ultimus ventricosus, infra sat celeriter et breve attenuatus, rotundatus, sutura descendens, apertura oblongo-ovata, superne obtusa, margine exteriore ab insertione leviter arcuato, basali rotundato, columellari superne dilatato et reflexo; long. 17-25, lat. 11 $-1 \cdot$, ap. long. 10-15, lat. 8-11 mm. - Suecia in Jemtland (ad Kall: E. N.). - Fennia ad Mjätusova prope flumen Svir et ad Seurujärvi (Mus. Helsingf.).

Var. fontinclis Stud. ap. Charp. (Cat. Suisse, 1837), forma fennica mh.: t. elongato-ovata, tenuis, cornea, spira producta, basi lata, anfr. convexiusculis. superioribus tribus minutis, ultimo a sutura convexo, basi sensim longeque attenuato, apert. orata, basi late rotundata, marginibus sat curvatis, columella acuta; long. 24-25, lat. 14-15, ap. lg. 19, lat. 11-13 mm. - Fennia in Kuopio in sinu Pitkälaks et ad Leväis* et Kallavesi: Mus. Helsingf.). Forma typica (in Helvetia et Bavaria) suturam antice obliquam et anfractus sursum depresso-attenuatos habet, talem apud nos non vidi.

Var. vulgaris C. Pfr. (Naturgesch., 1828): t. minor, conicoovata, tenera, argute striata, pallide v, rufo-cornea, spira brevis, acuminato-conica, anfi. ultimus medio sat convexus, sursum attenuatus, apert basi rotundata, superne angustata, columella panllo curvata, margine exteriore leviter arcuato; long. 10-15, lat. 8-10, ap. lg. 7-10 mm. - Passim.

Var. sericina C. G. W. ap. W. (Fauna Binnenconch. Suppl., 1890): t. ovata, pertenuis et fragilis, sericina, densissime striatula, et plicatulo-striata, flavido-brunnea, spira perbrevis, anfr. ultimus superne et medio perconvexus, infra attenuatus, apert. infra longe producta, margine exteriore forte arenato, columella curvata; long. 12-14, lat. 9-10, 
ap. lg. 9-11, lat. $6-7 \frac{1}{2} \mathrm{~mm}$. -- Snecia in Blekinge in Härsjön (ad Ronneby).

Var. amicola C. G. W. ap. W. 1. c: t. orata, dense striatula, aperturam versus sipe forte striata. opaca, fuscocomea. intus nitidissima, flavida, spira perbrevis, anfi. superi minimi, anfr. ult. tumido-convexus, superne plus minusve transversus, apert. magna, margine exteriore ab insertione forte arcuato, columellari curvato, albo-callosulo; long. 15 -18-20, lat. 10-12-13, ap. lg. 12-141/2--16, lat. 9-10\% $-11 \frac{1}{2} \mathrm{~mm}$. - Suecia in amne in urbe Romneby* et ad Kristianstad.

Var. subinfute W. (Nachr, blatt Mal. Ges., 1894): t. magna, tenuis, striata, spira basi lata, breve producta, forte acuminata, anfr. ult. elongatus, postice forte convexo-rentrosus, apert. subovata, magna, margine exteriore extense arcuato, subverticali; angulo profundo inter parietem et colunellam verticalem; long. 26-30, lat. 20, ap. lg. 18-23, lat. 12-15 mm. - Suecia ad Stockholm (E. N.).

Var. raboti W. (Nachr. blatt Mal. Ges, 1894): t. ovata, firma, sublevis, flavida, rimata $v$. sepius late perforata, spira brevi, late conica, obtusa, anfr. convexi, ult. maximus, super aperturam tumido-convexus, postice rentricosus, sutura lente descendens, apert. longa, anguste ovalis, margine exter. verticali v. infra paullo producto, leviter arcuato, pariete convexo, plica colum. obsoleta, columella longa, sub. verticali, reflexa; long. 20-22, lat. 15-16, ap. lg. 15-16, lat. $10 \mathrm{~mm}$. - Norv. in Nordlands Amt (Raneu) et in Ostfimmarken (Pasvig *) legit clariss. Ch. Rabot ut etiam in penins. Kola Russiar. Facie et forma bene cum fig. 1252 in Iconographia Kobelti congruit.

Var. lindströmi W. (Exk. Fauma, 1884): t. umbilicata, forma succiner magne elongatæ brevispirate, tenuis et fragilis, plicatula, cinereo-cornea, spira acute conica, anfi. 5, planati, ad suturam perobliquam subangulati, penult. sursum forte attenuatus, nltimus longus, medio late depressus, apert. basi rotundata, margine colum, late reflexo, exteriore medio stricto; long. 30, lat. 20, ap. 1g. 21, lat. $10 \mathrm{~mm}$. - Snecia in ins. Gotland (ad Klinta).

Var. obtusa Kob. (Malak. Blätt, 1870): t. apertura ampla, semicireulari, peristomate late limbato, columella obliqua, substricta, fere implicata, L. amplæ similis, sed spira late conica, obtusa, et margine superiore apertura horizontalidescendente; long. 22, lat. 20. ap. lg. 18, lat $12 \mathrm{~mm} .-$ 
Suecia in Östergötland. - Dania in Bornholm. Certe in pluribus locis.

Var. ampullacea Rossm. (Icon., 1835): t. inflato-ovata, tenuis, flavescens, subplicato-striata, spira brevissima, acuta, anfr. ultimus fere totam testam efficit, apert. late ovata, acuminata, peristomate recto; long. 16, lat. 1'́, ap. lg. 13, lat. $10 \mathrm{~mm}$. - Suecia passin raro a Skine ad lacum Mailaren. - Dania in Jylland (in Hastrup Skjernaa: A. F.). - Fennia in Barösund, Sammatti, Pyhäjärvi, Kropio (Savisaari), Gorki \&c. (K. M. L. et Mus. Helsingf.). I, ovata Nord. \& Nyl. Finl. Moll. t. IV. f. 43 a.

Forma patula Da Costa (Test. Brit,, 1778, Turbo), t. oblongoovata, firma, spira perbrevis, convexo-conica, apice punctiformis, sutura canaliculata, apert. superne acuta v. sinuata, pariete longo, columella brevi. margine exteriore leviter arcuato; long. 18, lat. 14, ap. lg. 16, lat. $10 \mathrm{~mm}$. - Snecia in Blekinge (Emmahultsjön). - Dania in Laaland. - Fennia ad Pyhäjörvi in Österbotten, ad Esbo et in Ảland ad Saltvik (Mus. Helsingf.).

Forma lacustris Leach in Syr. Moll. Brit. 1852. Beat. O. Mörch in Syn. Moll. Dan. p 41 huc refert formam ad Fuursö »in Sjælland Danie lectam» spira brevissima, apertura ampla, long. 11, lat. 8, ap. lg. 8, lat. $5 \mathrm{~mm} .{ }^{2}$, si non e vero nescio).

Forma hemispharica Küst. (Chem1. Conch. Cab., 1852), t. subglobosula, striatula, tenuis, spira vix prominula, anfr. 4 , convexi, ultimus ventrosus, apert. ampla, rotundata, margine exteriore valde arcuato; long. 10 , lat. 9 , ap. $\lg .9$, lat $6 \mathrm{~mm}$. - Snecia in litoribus ad ostium fluminis Götaelf.

Var. bulla W. (Nachr. blatt Mal. Ges., 1894): t. subglobosoorata, plus minusve aperte rimata, tenuis et fragilis, flavida, anfr. forte ventrosi, ultimus infra vix attenuatus, spira lata, brevis, obtusa, apert. ampla, marginibus valde arcuatis, exteriore superme descendente, lamina lata alba adpressa conjunctis; long. 20, lat. 15, ap. lg. 15. lat. 11 mm. - Fennia borealis ad lacum Enare (Ch. R.).

Var. balthica Lin. (Syst. Nat. X, 1758): t. subrimata, tenuis at firma, late ovata ad semiglobosa, striatula v. sipe rugosostriata, seriatim malleata et lineis spiralibus elevata flavescenti-cornea $v$. fusco-virescens, spira perbrevis, acntiuscula, sutura tandem ascendens, anfr. penult. superne subtransversus, ult. ventricosus, apert. magna, ovata, infra ampliata, pariete brevi, convexo, perist. subpatulo; long. 12-1i, lat. $7-10$, ap. $1 \mathrm{~g} .10-12$, lat. $6-8 \mathrm{~mm}$. - Suecia 
ad litora maris a prov. Bohuslän saltem usque ad Medelpad (i2 ${ }^{\circ}$ l. b.). - Dania ad litora insularum et peninsula. - Fennia ad Esbo (Mus. Helsingf.). Vagat hæc forma inter lagotem et ovatam, facie et characteribus nunc illi nunc luxic proxima.

Forma provalens mh.: t. limata, crassa, rufescenti-cornea, lineis spiralibus in utroque anfractu pluribus elatis, apert. margine exteriore regulariter arcuato; long. 19, lat. 13, ap. lg. $13 \mathrm{~mm}$. - Fennia, Esbo ad Lill-Löfö ad littora marina (Mus. Helsingf.).

Var. succinea Nilss. (Hist. Moll. Suec., 182-): t. subrimata, ovata, tenuis et fragilis, striatula v. lavis, fulvolutea, nitidissima, spira brevis, obtusinscula, anfr. penult. sursum attenuatus, ult. convexus, elongatus, apert. ovata, superne acuta; loug. 9, lat. $5^{1} / 2$, ap. $\lg .7-8$, lat. $\{\mathrm{mm}$. - Suecia ad litora ma. ris inter Skanör* et Carlskrona. - Dania ad Kristinehavn (ad arcem).

Var. steenstrupi Cl. (Malak. Blätt., 1878): t. tenuis, pulchre straminea, intus lactea, spira brevissima, obtusa, anfr. ult. ventrosus, t/5 longitndinis occupans. apert. elongato-ovata, peristomate continuo; long. 5-6, lat. 4, ap. lg. 'f, lat. 3 mm. - Islandia.

Et tamen restat magna copia formarum præcipue intermediarum.

L. peregra Müll. (Verm. Hist. II, 1774, Buccinum). T. saepius perforata, oblonga v. ovato-oblonga, sat solida, spira conica, crassa, sat elongata, obtusa; anfr. ult. oblongus, convexus; sutura profunda, antice descendens; apert. anguste ovata, sursum sensim attenuata, acuta. Long. 20, lat. 12, ap. lg. 12, lat. $9 \mathrm{~mm}$.

Hab. Suecia tota usque ad summum septemtrionem. Norv. tota usque in Westfinmarken. - Danic tota. - Fennic tola.

Forma labiata Rossm. (Icon. f. 5í), t. minor, apertura margine exteriore intus labio albo plano munito. - Sparsim ubique. L. marginata Auct. mult., non Mich.

Var. paludinoides Mörch (Syn. Moll. Dan., 186í): spir‘a subturrita, anfractus inflati; long. 23, lat. 14, ap. lg. 14, lat. 9 mm. - Dania Kjöbenhavn (in horto botanico).

Var. ambiguce W. (Öfrers. K. Vet. Ak. Förh., 1881): t. angustissime rimata, ovato-conica, corneo-fulvida $v$. alhescens, 
spira producta, angusta. acuta, valde contorta, sutmra profunda, anfr. convexi, supremi subangrulati, ultimus elongatus, apert. ovata, infra dilatata, margine superiore brevissime subhorizontali, columella late reflexa; long. 12-13, lat. 6-71/2, ap. lg. $71 / 2-8$, lat. $5-6 \mathrm{~mm}$. - Suecia in Skåne (Ö)tofta*), Upland (ad Gineo: V. L.) et Dalarne (ad Rättvik: E. N.). - Fennic ad Leväis prope Kuopio, ad EsboSumparn, nec non ad Warsuga in Lapponia rossica (Mus. Helsingf.).

Var. maritima Jeffr. (Brit. Conch. I, 1862): t. oblongo-elongata, solida, cornea, nitidula, spira producta, acuta, anfi. superi convexi, ultim. parum convexus, infra attenuatus, sutura sat profunda, apert. elongato-ovata, supra acnta, infra rotundata; long. 12, lat. 6, ap. $\mathrm{lg} .7 \mathrm{~mm}$. - Suecia in Öland (Borgholm). Taylor Journ. of Conch. 1891 p. 297 fig.

Forma contigua mh., t. marginibus aperturæ callo albo elevato contiguis. - Jemtland, Suecia (ad Offerdal: E. N.).

Var. meta W. (Exk. Famna, 1884): t. elongato-ovata, tenuis, pellucida, cornea v. rufo brunnea, nitidnla, striatula et densissime spiraliter lineata, spira conica, acuta, contorta, forte attenuata, anfr. convexi, apert. ovata, basi rotundata; long. 11, lat. 7; ap. $\lg .7$, lat. $4 \frac{1}{2} \mathrm{~mm}$. - Suecia in Blelinge (Romneby*, Nättraby etc,).

Forma firma mh., t. solida, crassa, nitidissima, fulvido-alba, spira brevis. - Suecia in Jemtland (ad Ostersund: E. N.).

Var. petronia W. Nachr. blatt Mal. Ges., 1893): t. rimata, solida, corneo-brunnea, passim cœrulescens, obsolete obtuse striata et sub lente forti densissime spiraliter lineata, nitida $\checkmark$. nitidissima, spira producta. forte contorta, acuminata, anfi. convexi, sutura profunda, ultimus a sutura tumido convexus, apert. ovata, margine columellari reflexo, albido-incrassatulo; long. 15, lat. $8-9$, ap. lg. lat, $6 \mathrm{~mm}$. - Suecia in Södermanland (ad Mölnbo in puteo vetero: d'A.) et Stockholm (ad Nacka: d'A.), in Östergötlaud ad Borghamn *: V. L.).

Var. alpicola W. (Nachr. blatt Mal. Ges., 1875): t. perforatorimata, elongato-ovata, crassa, solida, violaceo-cormea, albido-subnebulosa, strigis nonnullis albis et fuscis juxtajacentibus ornata, densissime striatula, sericina, spira attemato-conica, anfr, ult. medio planulatus, apert. oblongoovata, acuta, intus lutescenti-cornea, late ebmrneo-labiata, splendida; long. 19, lat. 11, ap. lg. 10, lat. $6 \mathrm{~mm}$. - Fennia ad Pargas (E. N.). 
Var. curta Cl. (Exk. Fauna, 1876): t. ovata, obesa, spira brevis, regulariter late conica, anfr. superi altitudine lente, latitudine forte accrescentibus, ultimus ventrosus. Magni. tudo varia. - Snecia in Skåne (ad Ringsjön).

Var. atrata Chemn. (Conch. Cab. IX): t. elongato-ovata, sub. cylindrica, spira elongata, crassa, obtusa, contabulata, anfr. ult. oblongus, partum conrexus, apert. oblonga, sat brevis, leviter corulescenti-labiata; long. 18-23, lat. 10-12, a]. lg. 10-1千, lat. 5-9 mm. - Suecia in Lule Lappmark ad Qvickjock solummodo supra regionem arbortum copiosissime (de Wallenb.), in provineiis mediis et meridionalibus magis magisque raro, ad Stocklıolm (Haga), in Östergötland (Skeninge), Blekinge (Romneby), Skåne (Hör) et in Öland (in stagnis in locis apricis Alvaren dictis). - Dania ad Svendborg.

Var: orthoplewa C. G. West. ap. W. (Famna Binnenconch. Suppl., 1890): t. tenuis at firma, corneoflavida, striatnla, in anfr. ultimo malleata et aperturam versus rugoso-striata, spira perbrevis, anfr. superi convexi, ult. superne subtrun. catus, medio planatus, postice convexus, apert, margine supero breviter horizontali, exteriore subverticali, stricto, basali arcuato; long. 19, lat. 11, ap. lg. 15, lat. $9 \mathrm{~mm}$. Suec, ad Stockholm (Dalarö: J. W.). Var. fulvæ L. proxima.

Var. recollata Anders. ap. W. (Öfvers. K. Vet. Ak. Förh., 1881): t. ovata, dense striatula, olivacea. nitida, anfr. convexi, ultimus tumido-convexus, spira brevissime conica, obtusissima, apert. ovata, margine exteriore et basali arcuatis, columellari late reflexo, columella sinuata; long. 8, lat. 5, ap. lg. $5 \mathrm{~mm}$. - Ad litora sinus Baltici inter $65^{\circ}-66^{\circ}$ lat. bor., Sneciæ ad Torneå, Fennix ad Uleåborg lecta.

Var. geisericola Beck (Amtl. Ber. Kiel, 18fi): t. ovata, striatula, corneo-rufescens, spira conica, acuta, $2 / 3$ longit. totius occupans, anfr. forte convexi, ultimus ventrosus, strigis nonullis brunneis, apert. intus fulva, nitida; long. 10, lat. $6-7 \mathrm{~mm}$. - lslandia.

Ille inter numerosas forma principes sunt.

3. Limnophysa Titz. T. oblongo-turrita, solida, spira longitutine aperture v. longior; anfr. ultimus convexiusculus, numquam ventrosus, margo aperture exterion an basin valde retrocedens.

L. palustris Müll. (Verm. Hist. II, 1774, Buccinum). T. imperforata, fuscocornea, strigis nonnullis intus albis extus fu- 
scis ornata, sat crassa et solida, densissime striatula; sutura perobliqua, sæpius alba; anfr. 6-7; apert. nunc longitudine spiræ, nunc paullo brevior, intus polita, flavida v. brunnea, labiis distantibus fuscovinosis. Long. 22, lat. 9-10. ap. $\lg .10 \mathrm{~mm}$.

Hub. Suecia meridionalis et media passim. - Norr. merid. et media rarius. - Dania sat vulgaris. - Fennia typus rarus.

Var. fusca C. Pfr. (Naturgesch., 1828): t. tenuior, fusca, ovatooblonga, tenuissime granulata, spira late conica, paullo longior quam apert., anfractus medio convexi, sursum attenuati, apert. intus plerumque fuscoviolacea; long. 15, lat. 7, ap. lg. $7 \mathrm{~mm}$. Variat major et minor. - Ubique vulgalis usque in Westfinmarken et summam Lapponiam Sueciæ et Fenniæ.

Var. flaidida Cl. (Corr. bl. Ver. Regensb., 1873): t. solida, corneo-flavida, striatula, nee non rugis tenuibus transversis et longitudinalibus, spira elongata, anfr. vix convexiusculi, apert. sæpe albolabiata et columella rufobrunnea; long. 15 -20, lat. 7-9, ap. 1g. 7-9, lat. 4-5 mm. - Passim.

Var. corves Ginel. (Syst. Nat. XII, 1788, Hx.): t. solida, elongato-ovata, sæpe irregulariter clathrato-costulata et malleata, fuscocornea v. nigrescens; anfr. 7-8, superi subplanulati, ultimi duo latissimi, spira conica, crassa, acuta, longitudine aperturx, quæe intus castanea, nitidissima; long. 24-35, lat. 12-15, ap. 1g. 13-17, lat. 7-9 mm. - Suecia prasertim in Skåne et in provinciis mediis. - Dania passim. - Fennia in par. Johannes (Mus. Helsingf.).

Var. corviformis Bourg. ap. Serv. (Malacol. Balaton, 1881): t. precedenti æqualis, sed spira gracile elongata, acuta, aperturam longitudine multo superante, anfr. tribus ultimis forte elongatis, sutura profunda; long. 35-43, lat. 14-18, ap. lg. 15-18 $\mathrm{mm}$. - Suecia in Östergötland (Skeninge), ad Stockholm (Löfholmen), \&c. - Dania in Jylland (ad Aalborg). - Fennia ad Uleåborg et Taipalsaari Nord. \& Nyl. Finl. Moll. t. IV f. 45), nec non in Aland, in palude Torphy (Mus. Helsingf.).

Var. litoralis mh.: t. elongato-clavata v. conico-turita, pallida, spira longiuscula v. longa, turrita, anfr. convexi, sutura profunda, v. superne subtruncati, ad suturam constricti, apert. anguste ovalis; long. 17-18, lat. 7, ap. lg. $6-7 \mathrm{~mm}$. v. long. 2t-26, lat. 9, ap. lg. $10 \mathrm{~mm}$. - Suecia et Fennia 
Acta Societatis pro Fauma ef Flora Fennica, Xll, n:o 7.

ad litora maris usque ad Torneå. - Dania subfossilis ad Meilgaard. - I. pal. v. maritima Cl. (nec Jeffr.).

Var. stenostoma W. (Exk. Fauma, 188i): t. umbilicata, sublanceolato-elongata, sursnm perangustata, anfr. 4\%/2, superi conrexi, ultimus transversim subangulatus, basin versus longe attenuatus, apert. angusta, margine columellari subrecto, reflexo; long. 7 , lat. 3 , ap. lg. $3 \frac{1}{2}$, lat. $1 \frac{1}{2} \mathrm{~mm}$. - Snecia in Skåne (subfossilis in paludibus turfosis ad Stenstorp).

Var. turicula Held (Isis, 1836): t. tenuis at firma, spira elongato-conica, acuminata, lougior quam apertura, anfr. leviter convexiusculi, lente accrescentes, sutura tenui; long. 20-24, lat. 9-13, ap. lg. 9, lat. $5 \mathrm{~mm}$. - Suecia passim rarior (optima ad Rosenlund pr. Jönköping). - Norv. in Fjeldfröskelv. - Fennia ad Kuopio (K. M. L). Hrec et var. litoralis sunt in Fauna Moll. Suee. (1878) sub nomine I. attenuatie Say var. desidiose Say (cui simillime) descripte.

4. Leptolimnaea Swains. T. cylindrico-turrita, spirct duplo triplove longior quam apertura, que sapissime labio albo nitido instructa est, margo exterior al basin forte retrocclens.

L. glabra Müll. (Verm. Hist. II, 1774, Buccinum). T. imperforata, tenuis, fuscocornea; anfr. 7-8, lente accrescentes, convexiusculi; apert. ovato-oblonga. Long. 9-10, lat. 3, ap. lg. 3-4, lat. $2-21 / 2$ mmı.

Hab. Suecia a prov. Skâne, ubi latius distributa, in provinciis occidentalibus (Halland, Bohuslän, Dalsland et Westergötland) non raro occurit; de catero rarissima in Blekinge (ad Carlshamn et Ronneby) et Småland (ad Kalmar). - Norv. passim, ut ad Kristiania (olim copiosissime ad Akershuus), Skudenas, Lillesand, Bamble, Sandefjord, \&c. - Dania in Sjalland (Gulskov: O. Mörch, Klampenhorg, Frederiksdal, Rüidershegn, Örderup: H. L.) et Jylland (Viborg: Feddersen).

Var. labiata W. (Exposé crit., 1871): t. brevior, elongato-conica, acuta, anfr. 6, infimus brevior et magis convexus, peristoma intus albomarginatum. - Suecia passim eum typo.

Var. elongata (Jeffr.?) W. (Exposé crit., 1871): t. subcylindracea, pallida, magis minusve nitida, spira quam apertura duplo longior, anfi. convexi, sutura sat profunda, alba, apert. superne angustata; long. 1i, lat. 4, ap. lg. 5, sp. lg. 
10 mm. - Norr. ad Bamble. - Dania ad Rudeshegn prope Holte.

Var. clavata W. (Fauna Binnenconch. V, 1885): t. minor, gracilior, turrita, acuta, v. subulato-conica, anfr. ult. cum spira comparatus multo major quam in typo, apert. parva, angusta, albolabiata; long. 8-10, lat. 3-4 mm, - Suecia rarissime (olin in stagno ad Ronneby lecta). L. subulata Anct. mult. (nec Kickx).

5. Fossaria W. T. elongato-ovata, spira acute conica, quam apertura paullo longior, anfr. ult. ventrosus, multo prevalens, apert. margine exteriore verticali.

L. truncatula Müll. (Verm. Hist. II, 1774, Buccinum). T. aperte rimata, fusca; anfr. 5-6, perconvexi, superne ad suturam profundam truncatuli; apertura ovalis, pariete sat transversali. Long. 9, lat. 4, ap. $\lg .4 \mathrm{~mm}$.

Hab. Sueciu tota usque in summam Lapponiam passim. - Norv. tota usque ad $70^{\circ}$ lat. bor. - Dania sparsim vulgagaris. - Fennia sat volgaris. Testa quamquam late distributa et suis locis vulgaris, regiones multie amplæeque sunt, ubi plane desideratur.

Var. labrosa W. (Exk. Fauna, 1884): t. minor, oblongo-orata, anfi. 5 , convexi, tres superi lentissime, ultimi celeriter accrescentes, sutura profunda, superne horizontalis, infra perobliqua, apert. sinu columellari profundo, margine colum. subrecto, reflexo, exteriore forte albolabiato; long. 5-6, lat. $2 \frac{1}{2}-2^{3} / 4$, ap. lg. $21 / 2-3 \mathrm{~mm}$. - Suecia in Skåne (ad Lund).

Var. mörclii W. (Exk. Fauna, 1884). t. firma, anfr. superne angulati, ultimus medio canaliforme impressus v, canali concavus; long. $7 \mathrm{~mm}$. - Dania in Vendsyssel.

Var. gothica W. (Fanna Binnenconch. V, 1885): t. profunde umbilicata, albida, spira turita, acuta, anfr. ult. ventricosus et dilatatus, superne angulatus, apert. elongato-ovalis, supra et infra angustata; long. 11, lat. $5 \frac{1}{2}$, ap. $\lg .5 \mathrm{~mm}$. Suecia in Östergötland (ad Lumna). L. tr. v. ventricosa W. olim (nec M. Tand.).

Var. major Moq.-Tand. (Hist. Moll. Fr. II, 1855): t. magna, pallida brumea, spira longa, anfr. perconvexi, sat celeriter accrescentes, ult. tumidus, superne sæpe angulatus, apert. orata, paullo ad dextrum obliqua; long. 15, lat. $8 \mathrm{~mm}$. Suecia in Skane passinn. 
Var. maximella Colb. (Matér. Faune mal. Belg., 1859): t. magna, solida, pallide brunnea, spira elongata, anfr. perconvexi, superi lente, ultimi duo celeriter accrescentes et infra elongati, apert. magis elongato-ovata; long. 11-14, lat. 51/261/2. ap. lg. 51/2-61/2 mm. - Suecia in Skåne, Östergötland, ad Upsala etc. raro. - Norv. ad Formo, Justöen. -- Fennia ad Kuopio (sec. K. M. L.).

Var. microstoma Drt. ap. Baudon (Moll. Oise, 1852): t. major, spira elongato-conica, anfr. perconvexi, sutura profunda, regulariter accrescentes, apertura margine columellari forte reflexo; long. $11-12$, lat. $4 \frac{1}{2}-51 / 2$, ap. lg. $41 / 2-51 / 2 \mathrm{~mm}$. - Snecia in Dalarne (Süter et Hedemora). - Norv. in Tromsö. - Dania in Bornholm, - Lapp. ross. Kaschkarantsa (sec. K. M. L.).

Var. goupeli Moq.-Tand. (Hist. Moll. Fr., 1855): t. ovato-conica, solida, striata, leviter malleata et lineis paucis spiralibus elevatis, interstitiis planatis, anfr. ult. superne angulatus, oblique truncatus, infra constrictus, superi medio angulatoconvexi, spira producta, sutura canaliculata, apert, anguste ovalis, supra infraque obtusa, margine exteriore medio stricto, columellari late reflexo, rima umbil. aperta; long. 11-13, lat. 5-61/2, ap. Ig. $5 \frac{1}{2}$, lat. $31 / 2 \mathrm{~mm}$. Variat minor. - Suecia in Dalarne (ad Säter) et Stockholm (inter Järfva et Sundbyholm: J. W.).

Var. schneideri B. Esm. (Tromsö Mus. Aarsh, 1882): t. aperte perforata, turrita, fuscobrunnea, rufescens, spira fere duplo longior quam apert, anfr. 6, celeriter accrescentes, ultimus superne depressus, infra subtruncatus, sutura profunda, apert. margine exteriore longe in anfr. penultimo affixo; long. 10, lat. $41 / 2 \mathrm{~mm}$. - Norv. in Westfinmarken (in Flöjfjeld).

Var. oblonga Puton (Moll. Vosges, 18.4): t. magna, basi angusta, sat solida, pallide brunnea, spira elongata, magis acutata, anfractus lentius acclescentes, parum convexi, apert. anguste orata, non labiata; long. ad 11 , lat. ad $41 / 2$ mm. -- Suecia ad Upsala.

Var. compressa B. Esm. 1. c.: t. elongato-turrita, flavidobrunnea, anfr. celeriter accrescentes, levissime convexiusculi, superne angulati, truncati, ultimus planulatus, apert. ovalis, superne rotundata; long. 10 , lat. $4^{1} / 2$, ap. lg. $4^{7} / 10 \mathrm{~mm}$. - Norv. ad Bergen. - Fennia in Kannusjärvi (sec. K. M. I., Mus. Helsingf.).

Var. lapponica W. (Famna Binmenconch. V., 1885): t. parva, gracilis, nitida, cornea, spira quam apert. longior forte con- 
torta, anfr. 5, superi lente accrescentes, perconvexi, ultimus leviter convexus, penultimo tantum $1 / 3$ longior, hic antice et postice, e latere visus, æque convexus, sutura perobliqua, apert. ovalis, margine colum. dilatato; long. $5-6$, lat. 2-21/3 mm. - Suecia in Jemtland et in Lappland ad arcum polaren. - Norv. in Nordland (in valle Vefsin: Ch. R.).

Var. minima Colb. (Matér. Faune Moll. Belg., 1859): t. minima, rufescenti-cornea, nitida, spira acute conica, anfr. 5, convexi, sutura profunda, ultimus ventrosus, penultimo duplo longior, hic antice (supra aperturam) valde convexus, postice subrecte constrictus, apert. rotundato-ovata; long. 5, lat. 3, ap. $\lg .2 \frac{1}{2} \mathrm{~mm}$. - Suecia in Skane (ad Lund), Dalarne (ad Norrby par. Hedemora), ad Upsala, Östersund (E. N.) et Piteă. - Fennia ad Rantasalmi (Mus. Helsingf.). Forma gracilis fere cylindraceo-oblonga, acuta, apert. oblonga; long. 6, lat. $3 \mathrm{~mm}$. - Suecia in Blekinge (Romneby) et Östergötland (ad Nyby). Var. microstoma W. olim.

\section{Subfam. Plyysina.}

T. sinistronsa, ovalis v. oblonga, nitida. Animal tentacullis longis setiformibus.

\section{Gen. Physa Drap.}

1. Bulinus Adans. T. ovalis, ampullacea, spira brevi, obtusa, anfractus 4, ultimus ventrosus, apertura ovalis. (Animal. margine pallii supra testam reflexo, 3-7-digitato).

Ph. fontinalis Lin. (Syst. Nat. X, 1758, Bulla). T. striatula. Hab. Suecia vulgaris usque in prov. Dalarne. - Norv. meridionalis et media passim. -- Dania vulgaris. - Femnia meridionalis et media et ad litora Sinus Fennici (K. M. L.).

Forma normalis, t. anguste ovalis, sæpe fuscofulvida, spira brevissima, apert. oblongo-ovalis, sæpe lanceolata, infia elongata, angulata, margine columellari longo, subrecto; long. 8, lat. $5 \mathrm{~mm}$. (ex. $\max$.)

Forma typica, t. ovalis v. ovata, pallide fulva, spira obsoleta, apert. ovato-oblonga, infra rotundata, superne angustata et acuminata, margine columellari brevi, curvato; long. 6-8, lat. $3 \frac{1}{2}-5 \mathrm{~mm}$.

Var. Zulla Müll. (Verm. Hist. II, 1774, Planorbis): t. late ovalis, spira conico-convexa, prominens, obtusa, anfr. ultimus 
magnus, ventrosus, ad suturam planulatus, obtuse angulatus, callus parietalis latissimus; long. 10-13, lat. $6-8 \mathrm{~mm}$. - Suecia in Skåne passim. - Dania ad Kjöbenharn, Frederiksdal et prope Aarhuus.

Forma grandis W., long. 20, lat. $13 \mathrm{~mm}$. -- Suecia ad Ronneby. Var. oblonge W. (Exposé crit., 1871): t. anguste ovalis, spira elevata, producta. - Dania in Jylland (ad Eveldrup).

Ph. semiglobosa W. (Exposé crit., 1871). T. late ovalis ad subcircularis, semiglobosa, fulvida, striatula, rugis incrementi numerosis iniqua; spira brevissima; apertura inferne rotundata, superne angustata. Long. $8-9$, lat. $6-6 \frac{1}{2} \mathrm{~mm}$. Hab. Succia ad Nacka* prope Stockholm. - Fennia ad fretum Halsholm in Ingo (Mus. Helsingf.).

2. Nauta Leach. T. ovato-oblonga, spira conica, acuta, anfractus 6. uttimus elongatus, apertura angusta, superne acuta. (Animal margine pallii simplici, non reflexo).

Ph. hypnorum Lin. (Syst. Nat. X 1758, Bulla). T. oblonga, subfusiformis, nitidissima, fulva v. rufescens, lavigata; spira longitudine aperture subequans. Long. 12-15, lat. 4-5, ap. $\lg .81 / 2-9 \mathrm{~mm}$.

Hab. Succia in Skåne (multis locis), Blekinge (solummodo in parte quam maxime occidentali), Östergötland (ad Lunna, Nyby, prope Omberg \&c.), Westergötland (ad Göteborg passim, Kinnekulle ad Mörkeklef et Hjelmsïter), Nerike (ad Örebro), Stockholm (passim rarior), Upland (ad Upsala), Westmanland (ad Westerås), Dalarne (Säter), Gotland (ad viam inter Fallingbo et Barlingbo). In Öland deest. - Norv. ad Kristiania, Ringebu, Justöen, \&c. - Dania in multis locis. - Fennia meridionalis non rara, specimima ad Tjudi, $62^{\circ} 30^{\prime}$ lat. bor. (J. Sahlberg).

Forma albescens Mörch (marmorata Gm.), Dania ad Præstö, Fuirendal et Sorö.

Forma achatina Gm. (Syst. Nat. XII, 1788), t. gracilis, nitidissima, fuscofulva. - Suecia ad Kristianstad. - Dania in Lolland (Höibygaard).

Forma minor, t. long. 7, lat. \& mm. - Suecia passim cum typo (ex. gr. Skine in Ringsjön, Östergötland in Tåkern, Stockholm prope Roslagstull \&c.) 
Var. perissiania Dup. (Catal., 1849, Ph. subopaca Dup. Hist. Moll. t. 22 f. 2, non t. 25): t. angusta, opaca, spira vix dimidiam aperturæe attingens; long. 10, lat. 4, ap. ]g. $7 \mathrm{~mm}$. - Suecia ad Kristianstad. - Dania in Sjælland (ad Frederiksdals Kro) et Laaland (ad Rye Mölle).

\section{Subfam. Planortina.}

T. Tiscoidea, spira plana, anfiactubus omnibus sape utrinque conspicuis. Animal tentaculis longis setaceis.

\section{Gen. Planorbis Guett.}

1. Spirodiscus Stein. T. magna (lat. 25-35 mm.), supra profunde concava, anfractus teretes, validi, apertura reniforme-rotundata.

PI. corneus Lin. (Syst. Nat. X, 1758, Helix). T. obseura; anfr. $4-5$, celeriter accrescentes, ultimus tumidus, dilatatus; apert. perobliqua, reniformis. Lat. $28-35$, alt. $12-15 \mathrm{~mm}$.

Hab. In Succia hæc species distributionem singularem habet: in prov. Skåne fere tota usque ad terminum quam maxime orientalem (Sissebïck), quaın paullisper tantum transit, sæpius frequens occurrit, deinde non nisi in provinciis circa Mälaren, ubi sæepe vulgaris, et denuo transilet provincias superiores usque ad Storsjön in Jemtland. In provinciis occidentalibus ut in tota Norvegia desideratur. - Dania tota vulgaris. - Fennia sat rarus, ad Lojo, ad Monrepos in Viborg, in Ladoga, Uguniemi, Kuopio inventus. (Pl. nordenskiöldi Bourg. Cir. W., Fauna Moll. Su. p. 373).

PI. elophilus Bourg. (Amén. Malac., 1859) var. ammonoceras W. (Exposé crit., 1871). T. pallida, anfr. 6-7, lente accrescentes, ultimus cylindraceus, non dilatatus; apertura parum obliqua, rotundata, parum excisa, v. ovali-lunata. Lat. 28-32, alt. $12-13 \mathrm{~mm}$.

Hab. Suecia in Skåne (Trollenäs, Svenstorp etc., Öland (Borgholm, Resmo* etc.), Gotland (in amnis ad Dede et Gothem, Mästermyr, Länne Ke.). - Dania in Sjalland et Bornholm passim. - Fennia multis locis, ad Pitkäjärvi in lacu Lojo, 
Myllykylä in Sammatti, ad Nygârd in Viborg, in Ladoga ad Kronoborg, Onega, in Neva ad Schliisselburg, Mus. Helsingf.).

PI. cryptomphalus nov. s]). T. inter congeneres parva, sat regulariter dense tenueque striata, albida v. fusco-comea, non spiraliter lineata, sat tenera, supra planiuscula, in centro ipso, anfractu interiore immerso, peranguste impressa, demum oblique intuenti profunde perforata, spira parva, subtus spira minima, medio cavatula; anfr. $4^{1 / 2}$, ultimus forte accrescens, ampliatus, supra convexus, peripheria rotundatus, subtus planulatus, pone aperturam planatus, ad suturam canaliculatam plus minusve obtuse anyulatus; apertura vix oblicua, subhorizontalis, sursum paullo versa, rotundata, parum excisa, margine superiore extense arcuatulo, exteriore forte curvato, basali subrecto, infra subauriculato, marginibus in pariete conjunctis. Diam. 10, alt. 5; d. 15 , a. $6 \mathrm{~mm}$. - Pl. stenostoma B. f. minor (l'l. similis Bz. p. p.) magnitudine et forma similis, sed t. supra late profundeque, regulariter et aperte infundibuliformeumbilicata, spira utrinque duplo major, anfr. ult. utrinque copaliter convexus, cylindraceus, apertura adeoque alia \&c. Hab. Danic in stagno quodam in Besperengen prope Kjöbenhavn (H. S.).

2. Tropidiscus Stein. T. mediocris (lnt. 10 -20 mm.), anfi. ultimus angulatus $v$. carinalus, aperture ovalis, extus angulata.

PI. umbilicatus Mïll. (Verm. Hist. II, 1774). T. cornea, fusca; anfractus 6, convexi, sat lente accrescentes, ultimus extus rotundatus, ad basin filiforme carinatus v. angulatus ( margo testac extra testam exseritur»: Lin.); apertura rotundatoovalis. Lat. $12-20 \mathrm{~mm}$.

Hab. Suecia sparsim vulgaris a prov. Skåne saltem usque in prov. Jemtland (ubi in fluvio liagundaelf copiose occurrit). Sunt tamen regiones magne (ut maxima pars prov. Blekinge) in quibus here testa ommino desideratur. -- Norv. meridionalis passim. - Dania vulgaris. - Fennia meridionalis el media (Knopio) et borealis (Arkangelsk). 
114 C. Testerlund, Synopsis Molluscorum Extramarinor. Scandin.

Forma filocinctr W. (Exposé crit., 1871), t. carina expressa filiformi, utrinque sulco impresso terminata.

Forma ecarinata $W^{T}$. 1. e., t. anfractu nltimo aperturam rersus tereti, carina evanescente.

Forma labiata IV. 1. c., t. apertura intus labiata, labio albo, nitido, sat valido.

Forma catina W. (Exk Fauna, 188. ), t. supra subplana, subtus plus minusve concara.

Monstrositates multæ (ut $\alpha$. t. suj)ra concava, subtus convexa; $\beta$. t. anfr. ultimo descendente, apertura centro adnatæ; $\gamma$. t. anfr. passim sejunctis, ultimo demum libero, etc.), sequentes quam maxime memorabiles:

1. T. pyramidalis, rufobrunnea, anfr. 6, lineares, superne truncati, ultimus fere acutangulus, convexus, apert. circinnata, marginibus conjunctis, columellari ad unbilicum profundum et apertum late reflexo; alt. 7 . lat ad basin $5 \mathrm{~mm}$. - Suec. ad Ringsjön in Skine. Woodward Manual of Moll. f. 97 optima.

2. T. pyramidalis, squalida, cinerea, anfr. 5, convexi, sibi invicem fere perpendiculariter impositi, 4 \& 5 , in ipso apice depressi, apertura circinnata ac centrum perforatum; "long. $2 \frac{2}{3}$, lat. 11/2 lin.» - Dania in fossa limosa ad Frederiksdal (O. Nörch). Nerita contorta Müll. in Verm. Hist.

PI. carinatus Müll. (Verm. Hist. II, 177f). T. calcarea, pallida; aufr. 5, celeriter accrescentes, ultimus dilatatus, extus compressus, acute carinatus; apertura anguste ovalis, acute angulata. Lat. $10-16 \mathrm{~mm}$.

Hab. Suecia in Skăne fere ubique, in Blekinge tantummodo in maxime occidentali parte (in lacu Wesan ad fammelstorp et in Sissebäik), Smilland (Jönköping, Westraby, Romelsjö), Öland (Resmo), Ciotland (Dalhemsâ par. Löjsta), Östergötland multis locis, Westergötland (in lacu Kopparsjön par. Mölltorp), Nerike (Skebäck et in Markkärret, Mellösa et ad Broby frar. Viby), Stockholm (in lacu Trehörningen par. Brännkyrka, Nacka), Upland (ad Upsala), Dalarne (par. Gustaf et ad Sïter), Melsingland (ad Bollnäs, Björktjärn). - Dania vulgaris. - Fennia ad Jalguba in Karelen, Räinä in Kuopio et Riistavesi in stagno Välisalmi (K. M. L.).

Forma nummularis Möreh (Syn. Moll. Dan., 1864), t. compressima, foliacea, inrequaliter flexa. - Dania rarior. 
Var. disciformis Jeffr. Linn. Trans. XVI, L830): t. plana, lutescens, carina mediana, anfractu ultimo magno, aperturam versus dilatato. - Snecia et Dania passim.

Var. turgidus W. Exposé erit., 1871): t. obscura, supra convexa, subtus plana, inferne carina tenui, anfr. rapide accrescentes, sutura profunda disjuncti, apert. rhombea sæpe labio lato lacteo mmita. - Suecia in Östergötland (ad Mjölsefall).

Var. dubins Hartm. (Nene Alp., 1821, Gasterop. Schweiz, 1S4́: t. fuscescens, sat firma, quam formæ typicce magis inflata et rotundata, carina submediana, apert, minus depressa, angulis minus acutis. - Suecia rarior (Småland ad Nybro etc.) - Dania in Nörreaa, ad Mors, Randers etc.

. D. Diplodiscus mh. (Gyrorbis Agass. 183i, non Fit:. 18.33). T. minor (lut. 3-10 mm), anfractus lente it. lentissine accrescentes, ultimus angulatus, apertura cordato-oratis r. rotimeleta.

PI. vortex Lin. (Syst. Nat. X, 1758, Helix). T. supra coneava, sublus planissima v. conrexiuscula; anfr. 7, convexi, subtus plani, ultimus penultimo duplo latior, ad suturam angulato-convexus, extus depressus, ad basin acute angulalus (»ipse testic angulus acutus est»: Lin.); sutura supra profunda, antice recta, subtus linearis; apert. transverse subcordato-lanceolata. Lat. 10-12 mm.

Hab. Sueciu per provincias plerasque (in Blekinge nonnisi ad termines Scanico) usque in prov. Dalarne, in Skâne sat vulgaris, de cetero passim. - Norv. ad Kristianial et Bergen. - Dania vulgaris. - Eennia saltem ad $64^{\circ}$ lat. bor., precipne in provincis meridionali-occidentalibus.

Var. compressus Mich. Complem., 1831): t. depressissima, tenuis, fragilis, anfiactu ultimo medio angulato-carinato. Passim.

Var. diseoides Reinh. (Nachr. blatt Mal. Ges., 1870): t. rufescens, srepe limo fusco obtecta, utrinque medio concarinscula, anfr, 5-6, supra convexi, sutura profundiuscula, subtus subplanulati, sutura leri, ultimus ad suturam convexus, ab initio decliris, omnino ad basin acute angulatus, subtus planulatus, apert. cordato-ovalis; lat. $4-5 \mathrm{~mm}$. Suecia ad Ultuna prope Upsala et in lacu Takern prov. i)stergötland. 
Var. discus (Parr.) Rossm. (Icon. f. 965, 1859): t. nitida, supra plana, subtus medio concava, anfr. 5, interiores utrinque medio obtuse angulato-convexi et suturam utrinque profundam versus declivi, nltimus pone ad basin obtuse angulatus, supra convexus et ad suturam subangulatus, extus ab initio saltem ad dimidiam subproruptus, subtus convexiusculus, apert. obliqna, panllo deflexa, vix cordata, peristoma lamella tenui continuum; lat. $5 \mathrm{~mm}$. - Suecia in Skogssjön prov. Östergötland; ad Thorsburgen in Gotlancl (V. L.). - Fennia in Luhanko, Sääminki, Kallaresi ad Kuopio in simu Fennico in Esbo, in Hogland \&e. (Mus. Helsingf.).

Var. goïsi W. (Exposé crit., 1871): t. utrinque æque concava. anfr. 6, primi utrinque convexi, ultimus penultimo duplo latior, supra convexus, subtus planus, antice deflexus, sutura utrinque impressa, apert. deflexa, subcordato-ovalis, marginibus callo junctis, extericre superne forte arcuato; lat. 6-8 mm. - Suecia in Östergötland (ad Mjölsefall et in Tåkern).

Var. poulseni W. (Malak. Blätt., 1874): t. supra late concava, subtus plana v. convexa, anfr. ult. extus depressus, acute carinatus, duplo latior penultimo, subtus dense striatus, omnes anfr. ad suturam profundam canaliculatam deelivi. apert. obtuse angulata; lat. $9 \mathrm{~mm}$. - Dania ad Kjöbenhavn" et in Jylland.

PI. vorticulus Trosch. (De Limneis, 1853, WV. in Mal. Bl., 1874). T. supra plana, subtus late profundeque concava; anfr. 5 , superi convexi, subtus convexiusculi, ultimus extus non depressus nee compressus, ad basin obtuse angulatus; sutura antice descendens; apert. ovalis, deflexa, extus obtusa, marginibus callo forli junctis. Lat. $3^{1 / t^{-}-4^{1} / t^{2}} \mathrm{~mm}$.

Hab. Suecia in palude bituminosa submarina baltica extra urbem Ystad in Skåne, subfossitis. - Daniu in Jylland (ad Proostlund: Cless., Horsens: H. L.), Möen et in Sjæelland (Krogerup et ad Arresö: H. L.).

PI. charteus Held (Isis, 18:37) var. bavaricus IT. (Malak. Bläitt., 187 f). T. utrinque plana ad concaviuscula; anfr. 5--51/2, utrinque convexi, sutura canaliculata antice recta, ultimus utrinque convexiusculus, penultimo paullo latior, periphe- 
ria compressuo et medio angulato-carinata; apertura ovalis, extus obtuse angulatus. Lat. $5-7 \mathrm{~mm}$.

Hab. Sueciu ad Kristianstad.

PI. spirorbis (Lin. fere certe!) Mlüll. (Verm. Hist. II, 1774). T. solida, rufo-comea, polita, utrinque medio concaviuscula; anfr. 5, lente accrescentes, supra perconvexi, sutura perprofunda, infra convexi, sutura profunda, ultimus dimidia parte latior, teres v. basi obsoletissime angulatus; apertura rotundata, intus albolabiata. Lat. $5-6 \mathrm{~mm}$.

Hab. Suecia passim a prov. Skåne saltem ad Dalelfven in Dalarne. - Norv. meridionalis (ad Arekilen, Kirkö, Hvalöarna). - Dania passim. - Istandia. - Fennia meridionalis.

Forma major W. (Fauna Suec, 1873 et Mal. Blätt., 1874), t. anfr. 6-7; lat. 7-s $\mathrm{mm}$. - Pl. septemgyratus Mörch et WV. olim.

Var. leucostoma (Poiret?) Mill. (Moll. Mains et Loire, 1813): t. supra in centro impressa, anfr. 6, lentissime accrescentes, supra convexi, subtus planulati, nltimus rix latior, ad basin obtuse angulatus, apert. ovato-rotundata, extus angulata, leviter labiata; lat. $5--6 \mathrm{~mm}$. - Distributio eadem ac typi, sed occurrit frequentior (in Blekinge ut typus rarissime) et in Fennia usque in Lappmarken (ad Knolajärvi).

Forma major. W. Fauna Binnenconch. V, 1885), t. major, anfr. 7, apert. elabiata. - Rarior. - Pl. septemgyratus Dup., Moq.-Tand., Bourg. Ec.

Forma perizii Graëlls ap. Dupuy (Hist. Moll. t. 25 f. 6, 1849), t. magis depressa, anfl. 7-8, angustissimi, ult. basi obsoletissime angulatus; lat. $6-7 \mathrm{~mm}$. - Rarior. Var. gracilis Gredl.

PI. dazuri Mörch (Amer. Journ. of Conch., 1868). T. utringue concaviuscula, solida; anfr. 5, sat celeriter accrescentes, utrinque convexi, ultimus dilatatus, rotundatus, penultimo duplo latior; apertura depresso-rotundata, intus valide albolabiata, marginibus callo forti junctis. Lat. $5-6 \mathrm{~mm}$.

Hab. Dania ad Rude et Fjurendal Skov prope Skjelskör (Steenbuch et H. L.).

4. Bathyomphalus Agass. T. parva (lat. $4^{1 / 2}-6 \mathrm{~mm}$.), sitpa in centro impressa, anfr. 6, anguste verticaliter com- 
118 C. A. Hesterlund, Synopsis Molluscorum Extramarinor. Scandin.

pressi, extus teretes, ultimus vix major, sutwa canaliculata, apert. verticalis.

PI. contortus Lin. (Syst. Nat. X, 1758, Hx.). T. subtus infundibuliformis (exeavatio xque lata ac profunda); apertura anguste lunaris. - Lat. $\AA^{1} / 2$, alt. $2 \mathrm{~mm}$.

Hab. Suecia tota, saltem usque in Lule Lappmark, ad $67^{\circ}$ $-68^{\circ}$ lat. bor., frecuens (prov. Blekinge, ubi rarior, excepta). - Norv. meridionalis et media. - Dania tota vulgaris. Femmia meridion. et media saltem ad $66^{1} / 2^{\circ}$ lat. bor. (in Tetrina in Lapp. ross.) sat vulgaris.

Forma major. W.. anfr. $81 / 2$; lat. $6 \mathrm{~mm}$.

Forma labiata W. (Exk. Fauna, 1884), apertura labio crasso albo munita. - Snecia precipue in regionibus calcareis (in Öland frequenter). - Norv. in Tangraldbek in Bambla.

Var. difformis WT. (Fauna Binnencouch. V, 1885): aufr. 6, intus truncata, extus depressa, ultimus dilatatus, ad suturam obtuse angulatus, deinde leviter impressus, in peripheria distiucte angulatus, apertura semiorata; lat. $4^{1} \frac{1}{2} \mathrm{~mm}$. Suecia in lacu Tåkern (ad Nyby) prov. Östergötland.

PI. dispar IV. (Exposé crit., 1871). T. subtus medio concava (excavatio multo latior ac profunda); anfractus subtus multo angustiores quam in pricedente specie, ultimus antice paullo dilatatus; apertura rotundato-reniformis. Lat. $4^{1 / 2}$, alt. $1 \frac{1}{2} \mathrm{~mm}$.

Hab. In Suecia a Jemtland (Östersund: E. N., Krokon: P. Olsson), Nledelpad et Helsingland descendit per provincias medias passim (passim frequens, ex. gr. in piscina cyprinacea ad Forsmark in Roslagen, in hortu pastoris in Norra Bäckebol: in Dalsland etc.) et magis magisque rarus usque ad Blekinge (Ronmeby). - Norv. ad Tangvald (?) et ad Vestra Aker pr. Kristiania (Höijer in coll. H. L.). - Donia in Sjælland (ad Fumrsö: H. L.).

5. Gyraulus Agass. T. parva (lat. 5-- 8 mm.), sape hispidu $v$. clathrata; anfi. $1-5$, plus minusve coleriter accrescentes et depressi, ultimo majore; apertura horizontalis, obliqua, ovalis.

+ Spira para; anfractus celeriter acrescentes, ultimus maynus, forte dilatatus. 
* T. utrinque in medio punctiforme immersa, "utrinque aque umbilicata" Miill.).

PI. albus Nü̈ll. (Verm. Hist. I1, 177'). T. albida, clathrata, "supra planiuscula, subtus convexa» (Müll.); anfr. $31 / 2^{--1}$; apertura depresso-rotundata, peroblicjua ("pars superna ultra inferam valde extensa" Müll.), expansa, subdeflexa. Lat. $3-4^{1} / 2 \mathrm{~mm}$.

Hab. In Succia typus Mïlleri valde sporadice oceurpit et locis tantum paucis, sed in is more congenerarum frequenier inventus est. Locus fuam maxime borealis a fuo typum vidi est Bollnäs in Helsingland. - Norv. etiam sparsim oceurrit circiter ad Dovre usque (62 lat. bor.). - Dania, ubi locus originalis samnis Fredrichsdalensis" prope Kjöbenhavn (Müller). Femia in Aland (Finnby), ad Fredrikshamn, in Impilahti ot ad Kínopio (Mus. Helsingt.).

Var. hispintus Drap. (Tabl. Moll., 1801): t. tenuis, albescens, spinulis fiagilibus et caducis, armato oculo elongato-conicis in lineis spiralibus hispida, apert. larga, ovali rotundata; lat. 4-5 mm. -- Suecia et Norvegia passim. - Dania - vulgaris in aquis stagnantibus" (Pl. albus Mörchl). - Femnia mericlionalis, omnium sui generis forma maxime distributa (fide Nort. \& Nyl.).

Var. cinctutus W. (Exposé crit,, 1871): t. tomento albescente ubique tecta, pallida, tenera, subtus concaviuscula, anfi. 4- $\mathbf{1}^{1} / 2$, ultimus depressus, utrinque convexus, medio sub. angulatus et sæpe carina membranacea debili cinctus, apert. transterse ovali-rotundata; lat. $6-7 \mathrm{~mm}$. - Suecia in Wermland (ad Carlstad *: Cl. L.), in Jemtland (ad Krokom: P. Olsson). - Norv. ad Kristiansand, Arendal, Ramnäs, Sandetjord, Moss, vulgaris in Österdalen et frequens in fluvio ad Ringsted, Hedemarken (fide B. Esmark).

Var. alatus W. (Exk. Fauna, 1881): t. cristis membranaceis pluribus parallelis spiralibus cincta. - Suecia in Skăne (in Rönneå ad ()ja).

Var. spurius mh.: t. costulato-striata, sublenticularis, extus utrinque equaliter forte compressus, medio acute angulatus, anfr. primi teretes, angustissimi, ultimi tho pone snturam profundam angulati, intus prerupti, ult, antice dilatatus, apert. ovata, perobliqua, marginibus callo forti conti- 
120 C. A. Westerlund, Synopsis Molluscorum Extramarinor Scandin.

muis; lat. $5 \mathrm{~mm}$. - Dania in Sjolland (Faarup Sö ad Veile: H. L.).

**) T. subtus concava.

PI. socius W. (Öfvers. K. Vet. Ak. Förh., 1881). T. clathrata, tenuis, virescenti-cornea; anfr. 4, ultimus superne obtuse angulatus, extus depressus, peripheria compresso-rotundatus, subtus convexus, antice descendens; apertura perobliqua, rotundato-ovalis, narginibus tenue junctis. Lat. 4-$5^{1 / 2} \mathrm{~mm}$.

Hab. Succia in Skåne (Örtofta), Blekinge (Ronneby, in rivulo ad Torneryd *), Roslagen (Söderby: P. de L.), Södermanland (Dalbyö: E. N.), Stockholm (Alviken: J. W.), Helsingland (Arbrö: E. C.).

PI. arcticus Beck (Index Moll., 1837). T. non clathrata, corneoflavida v. albida; anfr. $3 \frac{1}{2}$, celerrime accrescentes, convexi, sutura canaliculata, ultimus rotundatus, obsoletissime angulatus; apert. obliqua, hippocrepiformis, marginibus callo albojunctis. Lat. $5 \mathrm{~mm}$.

Hab. Suecia in Pite Lappmark (ad Jörn: E. H.) et in Jemtland (ad Östersund: E. N.). — Norv. in Tromsö stift. - Fcnnia in lacuna ad Kuopio (Mus. Helsingf.).

it Spire sat magna; anfractus sat irregulariter accrescentcs, ultimus magnus, sed non dilatatus.

*) T. non $v$. obsolete clathrata.

PI. strömi W. (Öfvers. K. Vet. Ak. Förh., 1881). T. firma, rufobrunnea, utrinque subplana, medio impressa, dense striata; anfr. $4^{1} / 2$, penultimus antepenultimo triplo latior, ultimo $1 / 8$ angustior, ultimus compressus, medio obsolete angulatus (interdum membrana prieditus); apertura perobliqua, rotundato-ovalis, marginibus callo forti, interdum soluto, conjunctis. Lat. $71 / 2-8 \mathrm{~mm}$.

Hab. Suecia in Wermland (ad Carlstad) et in Lappland sparsim. - Norv. ad Eker (in Rökkebjergstjern). - Fennia in Österbotten (Uleåborg et Nuottasaari) et ad Kittilä in Lappland (Mus. Helsingf.). 
PI. borealis (Loven) (I. Malak. Blïtt., 1874). T. rufobrunnea $\mathrm{r}$. cornea, supra in medio immersa, subtus umbilicata. firma. dense striata; anfr. $\hat{t}^{1}{ }_{2}$. celeriter accrescentes, regulariter convexi, penultimus inflatus, citeris altior, ultimus duplo major, utrinque subcqualiter conrexus, antice strictus; apertura parum obliqua, rotundato-oralis; late sed leviter albolabiata. Lat. $6-\overline{\mathrm{t}} \mathrm{mm}$.

Hal. Sueciu in Lapponia tota sat frequenter, nec non in Jemtland, Herjedalen et Helsingland. - Nort. fere tota usque ad Varangerfjord vulgaris. - Femia media et borealis in Lapp. ross. (Kusräka $66^{1} 2^{\circ}$ lat. bor., Mus. Helsingf.). - Subfositis in fossis turfosis Scaniu meridionalis (ut ad Stenstorp) frequenter. - Sin. Pl. rossmassleri $\mathbb{1}$. olim in Exposé crit. et Fauna Suec.

Forma mino: TI.. lat. 5-6, alt. $1_{2}^{12} \mathrm{~mm}$. - Suecia in Dalarme ad Aresta. Orsa et in Ljusterån prope Säter: C. G. A., in Wermland (ad Car'stad), Upsala (P. de I.) et Östergötland Eriksberg in Srartgölen .

Tar. amigyius W. (Exk. Fauna, 18ś) : t. dense et temue striatula, anfractubus perangustis. - Torr ad Hole et Clreöen in Stensfjord.

Tar. frigidus W. (Famna Binnenconch. pal. Reg. T, 1soj: t. major. tenuis et fragilis. pallida (albida r. virescens. supra in centro rix impressula, subtus leriter concara. striatulia et temissime spiraliter lineata, anfi. $i^{1}$, infimi lente regnlariter, ultimi celeriter accrescentes, supra convexi, subtus conrexiuseuli, penultimus vix 5 . non altitudine ultimi, apert. non labiata, sæpe horizontalis; lat. $\bar{\tau}-10 \mathrm{~mm}$. Suecia borealis passim in Lappland, Härjedalen et usque in Helsingland ad Arbrå: E. C.) - Torv. passim, ut in Tromsö stirt Ch. R.' et Osttinmarken. - Femmia ad Enareträsk * frequenter et maxima 'A. W. M. et Ch. R.).

Tar. reflexus TI. Fauna Binnenconch. pal. Reg. T, 1s85): t. subtus profundius latiusque concava, anfr. ultimi celeriter accrescentes, ultimus sat compressus, supra convexior, antice profunde forteque descendens, apert. perobliqua, tepressa, cordato-orata, callo continua; lat. 6-10 mm. Nor'v. ad Modum.

P. gredleri Bz. ap. Gredl. (Tirols Moll. II, 1859\%. T. solida, lutescenti-cornea, sape sordide obtecta, supra in medio 
122 C. A. Westerlund, Synopsis Molluscorum Extramarinor. Scandin.

immersa, subtus umbilicato-concava, transversim miscue subtiliter et fortius striata, vix conspicue clathrata; anfr. 4, regulariter accrescentes, convexi, ad suturam profundam obtuse angulati, nlt. lente accrescens, antice descendens, supra multo convexior, subtus planulatus, siepe inter lineas 2 -3 elatas spirales tenue malleatus; apert. oblique ovalis, sape tenue albolabiata et subtiliter nigrolimbata. Lat. $5-6 \mathrm{~mm}$.

Hab. Suecia in Pite Lappmark, Jemtland et Herjedalen. - Norv. ad Kristiania (frequenter), Drammen, Laurgaard \&c. PI. glaber Jeffl. (Trans, Linn. Soc., 1830). T. colnea, tenue striatula, subtus fere plana; anfr. $41 / 2 \ldots 5$, sat celeriter accrescentes, depresso-teretes, ultimus penultimo multo latior, fere cylindraceus, antice rectus; apert. vix oblicpua, rotundata, marginibus conjunctis. Lat. $4-5 \mathrm{~mm}$.

Hab. Suecia in Blekinge (Ronneby), Gub. Calmas (kjelltorp par. Förlösa), Dalarne (in stagno ad Alkroken et in lacu Ljustern: C. G. A.). - Norv. ad Kristiania el Bergen. - D niu in Sjalland (Kjöbenhavn ad V'estervold, ad Nixsö), Fyen frequens, Bornholm. - Istandia. - Fenmia in Kallavesi ad Kuopio et Hirrolaks (K. M. L.).

**) $T \cdot$ clathrata.

Pl. draparnaldi Shepp. (Trans. Linn. Soc., 1828). T. tenuis, albido-connea, lineis tenuibus elevatis spinalibus, subtus umbilicata; anfr. $4^{1} / 2$, primi sat celeriter accrescentes, ultimus latus, sed demum lentius accrescens, depressus, medio et ad suturam profundam obtuse angulatus; apert. ovalis, basi subcordata, extus rotundata, perobliqua. Lat. $6-6^{1 / 2} \mathrm{~mm}$.

Hab. Succia in Skåne (Hjelmslund), Blekinge (Ronneby), Westergötland (Borås), Wermland (Carlstad), Westmanland (Olofsfors). - Norv. in Jödderen. - Damia in Sjalland (ad Sterrede). - Fenniu in Satakunta ad pagum Tehmalais (Mlus. Helsingf.).

PI. limophilus $\mathrm{W}^{\top}$. (Malak. Blält., 1867). T. corneo-lutescens (animale foeta coccineo-rufa), striata et lineis spiralibus tenui- 
bus clathrata, supra in centro paullo impressa, subtus latins umbilicata; anfr. $41 / 2-5$, primi lente, ultimi duo celeriter accrescentes, omnes utrinque aqualiter convexi, sntura utrinque profunda, ultimus penultimo duplo latior, peripheria rotundatus; apert. depresso-rotundata, valde oblicua, margine columellari valde excavato. Lat. $5 \mathrm{~mm}$.

Hab. Succiu in Skåne (Kristianstad, Bosarp), Blekinge (Romneby in Herrstorpsjön *), Westergötland (Borås), Uplind (U1tuna), Norrland (Sandön ad Piteâ), Lappland (Hluoniowaaral ad $68^{\circ}$ lat. bor). - Norv. ad Valders et in Ciudbrandsdalen. Fennia ad Kuopio et Uleảborg (Mus. Helsingf.).

PI. concinnus IV. (Öfvers. K. Vet. Ak. Förh., 1881). T. brunnea, tenue clathrata, supra sat profunde in centro immersa. subtus subplana v. medio leviter depressa; anfr. $4-5$, inferi lente accrescentes, utrinque forte convexi, teretes, sutura profunda, ultimus fortius accrescens, regularis, utrinque ixque convexus, superne obtuse angulatus, extus depressiusculus, medio carina membranacea pricditus; apert. cordato-ovalis, perobliqua. Lat. $5 \mathrm{~mm}$.

Hub. Sueciu in Skâne (Hjelmslund), Blekinge (Ronneby ad ammem pr. cataractam:), Öland (Borgholm, rarissime, nullus alius Gyraulus in insnla inventus), Dalarne (Wiabürk), Wermland (Carlstad), Westmanland (Olofsfors). - Norv. in Öjer, Ringebn ete. - Femia ad Nikola pr. flumen Svir et in Yläne ad laterariam Tourula (Mus. Helsingf.).

Var. gothicus W. (Exposé crit., 1871): t. supra in medio mmbilicata, subtus omnino plana, tenuissime clathrata, anfi. sat celeriter accrescentes. omnes tantum subtus visibiles, ubi convexi, sutura canaliculata, ultimus carina tenni in medio, apertura perobliqua; lat. $5 \mathrm{~mm}$. - Suecia in Westergötland (ad Pirkum" par. Sandhem et ad Mösseberg).

PI. correctus mh. T. supra plana, medio levissime depressa, suJ)tus medio umbilicata, striata et leviter clathrata; anfr. $\tilde{5}$, utrinque forte convexi, priceipue antipenultimus, lente legulariter accrescentes, ultimus cylindricus, penultimo vix $1 / 4$ latior, superne in suturam profundam declivis, antice descendens; spira orbitu magna (major quam apud omnes affi- 
124 C. A. Westerlund, Synopsis Molluscorum Extramarinor. Scandin.

nes pracedentes, minor (fuam sequentium); apertura rotundata, parum obliqua, perparum excisa. Lat. $5 \mathrm{~mm}$. Hab. Fennia ad Harjula in Kuopio (K. M. L.).

Pl. cochlearis mh. I'. supra convexiusculo-plana, subtus late concava, tenue spiraliter lineata, precipue subtus forte striata; anfr. 5, primi lente accrescentes, penultimus antepenultimo plus quam duplo latior, ultimo vix $1 / 3$ minor, convexiusculi, ult. utrinque convexiusculus, infra medium angulatus; sutura angustissima, canaliculata; spira maxima ; apert. rotundata, parum obliqua, marginibus callo conjunctis. Lat. $6^{1 / 2} \mathrm{~mm}$.

Hab. Suecia in Jemtland (ad Östersund (E. N.).

PI. gelidus mh. T. utrinque medio aequaliter sat profunde concava, pulchre densissime striata et lineis tenuibus dense clathrata, firma, rufobrunnea; anfr. 4, teretes, valde convexi, sutura profunda disjuncti, primi lentissime, nltimi duo forte accrescentes, ultimus subteres, non dilatatus, nec descendens; apert. ovato-rotundata, intus forte albolabiata, marginibus callo junctis. - Lat. $1--5 \mathrm{~mm}$.

Hab. Suecia in Jemtland: P. Olsson (Mus. Regni. Holmice).

6. Armiger Hartm. T. parea (lat. 2-4 mm.), subtus late ditatate umbilicata, anfi. 3, celerime acerescentes. nltimus compressus, angulatus, apertura obliqua, dellexa, rotunclato-ovata, marginibus in latere testo inferiore contiguis.

PI. crista Lin. (Syst. Nat. X, 1758, Nautilus). "T. apertura or. biculata, anfractibus contignis, articulis annulatis. dorso spinosis» (Lin, l. c.), tenuissima, albida v, pallide fulva. Lat. $2 \mathrm{~mm}$. - Pl. crista r. spinulosus Cl.

Hab. Suecia in Skîne (ad Malmö et Lund).

Var. imbricatus Mïll. (Verm. Hist. II, 177í): t. alba, pallicla v. cinerea, lineis transversis ligamentorm instar elevatis cincta, bie in margine prominentes carinam denticulatam recldunt. (Miill. 1. c.); lat, 2-21/2 $\mathrm{mm}$. - Suecia sparsim a Skåne usque ad Pitea in Lappland, multis autem locis (ut in Blekinge) rarissimus $v$. desideratur. - Norv. ad Kristiania, Fredrikstad et Skien. - Dania in Sjælland et Jylland rarus. - Fennia meridionalis et media (ut ad Hirvolahti in Kuopio: K. M. L., Helsingfors et Borgå: Mus. Helsingf., Uleăborg', \&c,).

Pı. nautileus Lin. (Syst. Nat. XII, 1767, Turbo). "T. plinniuscula, 
anfractibus anmulatis dorso cristatis (Lim. l. c.), solidula. cornea. tenuissime striata et obsolete costulata. margine obtuso lievis. Lat. 3-1 nmm.

Destributio eadem ac imbricatus, sed multo sirpius nccurit, tamen etiam ut ille sparsim.

i. Hippeutis Agass. T. parla (lnt. 3-in mm.). lentirularis. anfiactus 3-t. repressissimi, ultimus forte ampliatus, apertura repressa. horizontalis. subcordata. maiginibus pariete intrante disjunctis.

PI. fontanus lightf. (Phil. Trans., 1786, Helix). T. unbilico angusto, centrali. circulari, profundo, anfractus parum pratbente; apertura perohliqua (margine exteriore fortissime rotundate producto. columellari medio recto, horizontali), anguste extenseque cordata, pariete valde intrante excisa. extus angulata; anfr. $4-4^{1}$,2. priores lente accrescentes. ultimus extus utrinque subiequaliter compressus. fere medio angulatus. L. 4 -6 $\mathrm{mm}$.

Hab. Suecia meridionalis et media saltem in pror. Mestmanland. - Tor $r$, ad skien, Arendal. Moss. - Drmir tota passim. - Ecmira ad Emkarby in Aland (Nus. Helsingf.).

Tar. cuptucess Bourg. Malaǩol. dlgér., 156ı́): t. spira et umbilico angustioribus, anfr., uitimo supra depresso-convexo, all basin angulato, infra fere plano. - Suecia ad Ronneby. - Dania in Sjwlland ad Holsteinborg.

Ous. Nullo modo dubito, quin Helix complanata Lin. Syst. Nat. I et Fauma Suecia ed. 2 ) huc referenda sit ( t. parra admolum, subcinerea, subdiaphana, tenuis. supra conrexa, subtus plana omuino, margo qui anfractus cingit; respicit latus planius s. inferius, apertura semicordata, orato-acuminatas). sed melins ad varietatem euphoum quan al tryum, adeoque nomen Lightforti adoptari. Auctores sunt plures, qui in hac specie magnum firmum Pl. umbilicatum Müill. Hs. plauorbem Lin.) riderunt. $\mathrm{Cl}$. Hanley (in Ipsa Linnæi Conchrlia) nomen Linnæi rexarit. quod in collectione Linnæi species plures sub hoc nomine congregatip sunt. Qvid inde? Sic in ommi collectione, et mirabile sane esset si non in illa, post discremina quæ habuit. Experientiam candem amiciss. Locard pluries in collectione Draparnaudi in Wien asservata, de qua nuperrime (in Ipsa Draparnandi Conchylia, Paris 1A95) magna eruditione tractavit, fecit. Ergo, de speciebus dubiis non precipue e collectionibus auctorun relictis, ne quidem e speciminibus au. thenticis e rariis temporibus, sed e descriptionibus eormu et e 
locis originalibus testarmm judicandum sit. Sic multoties loca originalia testarum a Linnæo citata loca obscuxa in scriptis suis enodarunt.

PI. riparius W. (Sver. Moll., 1865). T. lutescens, transverse eleganter striala, umbilico lente dilatato omnes anfractus optime prabente subtus late concava; apertura sat oblique cordato-elliptica, extus perobtusa, pariete paullisper excisa, margine exteriore leviter arcuate producto, columellari arcuatulo; anfr. $3\left(3^{1} / 2\right)$, ultimus utrincae aequaliter planatus. Lal. ¿3 $\mathrm{mm}$.

Hab. Succia in Skåne (ad Kristianstad et in Lingsjön ad Bosjökloster), bilekinge (ad Ronneby in Herrstorpsjön *), Westergötland (ad Göteborg), Upland (ad Ultuna et in silva ad Nordby: II. L.), Öland (ad Kohlstad prope Borgholm, nec non ab amiciss. A. Ahlrot inter Halltorp of Woxtorj). - Dania in Sjalland (Fumrö, Sorösö, Donse Dam, Frederiksdal, ad Arresö: H. I.).

Pl. leptostoma $\mathbb{W}^{\mathrm{T}}$. (Nachr. blatt Mal. Ges., 1894). T. carneobrunnea, lineis transversis et spiralibus tenuibus sub lente densissime clathrata, umbilico forte dilatato anfractus bene prebente modice concaviuscula; apert. parum obliqua, oblongo-ovata, basi subcordata, marginibus subrequaliter arcuatis, cxtus rotundato-obtusa; anfr. $3^{1 / 2}$, ultimus supra ab angulo perobtuso suturam propriore intus extusque declivus, subtus convexiusculus. Lat. $3 \mathrm{~mm}$.

Hub. Fennia ad Rantasalmi (Mus. Helsingf.).

8. Segmentinus Flem. T. parva (lat. $5-8 \mathrm{~mm}$.), apertura perobliqua, cordata, fauce in omne cetute lamellis ternis (1 prictali et 2 lateralibus) translucentibus coarctata.

PI. nitidus Mïll. (V'erm. Hist. II, 1774). T. supra convexa, medio subplana, subtus plano-convexa, medio sat late umbilicala, nitidissima, rufofulva; anfr. 4, celeriter accrescentes, ultimus ad basin obtuse angulatus, subtus a margine intus declivis; apert. late cordata, depressa Lat. $5-6 \mathrm{~mm}$. Hab. Srecia in Skåne et Blekinge multis locis, Öland (ad Stora Dahlby), Småland (Jönköping, UTlfsborg, W†ärnanäs), Hal- 
land, Nerike (inter Åkerby et Äkerbytorp), Södermanland, Stockholm (ad Nacka: (i. Eisen), Upland (Upsala: IT. L.). - Norv. ad Bergen (fide Friele). - Dania tota. - Fonnia merid. (Aland, Helsinge, Sordavala, Saimen ete.).

Var. clessini W. (Fauna Snec, 1873): t. convexinscula, medio impressione punctiformi, subtus plano-convexiuscula, medio anguste umbilicata, spira parva ( $1 / 4$ lat. totius), anfr. ult. utrinque extus subequaliter compressus, margine snbmediano acuto, subtus ab umbilico extus declivis, apcrt. elongato-cordata; lat. $7-8 \mathrm{~mm}$. - Stecia in Skine, Små land (in Lillsjön* ad Jönköping), Upland (ad Forsmark in Roslagen), (O)and (ad Borgholm). - Dania in Bornholm. Var. oclandicus W. (Fauna Binnenconch. pal. Reg. V, 185.5): t. supra convexa, foveola in medio, subtus subplana, anguste sed profunde umbilicata, spira angustior quam anfiactus ultimus, anfr. $\mathfrak{1}^{1 / 2}-5^{1 / 2}$, superi immersi, perangusti, exteriores dno celeriter accrescentes, vix convexiusculi, sensin extus declives; lat. $51 / 2-61 / 2$ mm. - Snecia in Öland (Borgholm in Kungsträdgården *).

\section{Subfam. Ancylina.}

T. patelliformis, alsque spira.

\section{Gen. Ancylus Geofir.}

T. depresso-lateque conoidea, apice verticis supissime submediano, reflexo.

1. Ancylastrum Moq.-Tand. T. sinistralis, mitraformis, corneo-calcarea, late conoidea, intus nitida, apice paulisper ad dextrum.

A. simplex Bue'hoz (Aldr. Loth., 1771). T. ovata, postice distincte angustior, concava, ad $1 / 3$ totius longitudinis extra apicem extensa, supra antrorsum extense convexa, lateribus convexiusculis, subtiliter radiatim striata, apice a margine posteriore remoto; apertura rotundato-ovata. Long. 5, lat. 4, alt. $2 \frac{1}{2}-3 \mathrm{~mm}$. (A. fluviatilis Müll., 1774).

Hab. Suecia meridionalis et media usque in Dalarne. Norv. ad Kristiania, Sandefjord, Asker in fluvio ad Blakstad, Jicdderen et Noss. - Dania tota sparsim. - Femia sat fre- 
quenter in parte meridi-orientali, non supra Tjudie in Karelen inventa.

Var. cornu Cless. (Chemn. Conc. Cab., 1882): t. firma, altior, lateribus plus minusve depressis, antice convexa, apex posterior, marginem posteriorem fere superjacens, sæpius tamen erosus, apertura elongato-ovata. - Suecia in Skine (ad Lund). - Dania in Lolland (Bjerremark).

Var. westerlundi Cless. 1. c.: t. sat firma, antice convexior, apex minimus, acutus, obsolete obliquus, apertura rotundatoovata; long. 6, lat. 5, alt. $3 \mathrm{~mm}$. - Suecia ad Fogelsång prope Limd.

Var. subcircularis Cless. (Mal. Blätt, 1880): t. orbitu elliptico circularis, depressa, tenuissime radiatim striata, antice convexa, postice vix concava, lateribus rectis, apice dextrorso, apertura marginibus dilatatis; long. 5, lat. 4 , alt. $21 / 2 \mathrm{~mm}$. -.- Suecia in Skåne (in lacu Ringsjön ad Klinta).

Var. gibbosus Bourg. (Baud. Mém. d'Oise, 1852): t. elliptica, postice subxquale rotundata, supra forte convexa, inflata, postice concava, lateribus modice convexis, apice vix obliquo, marginem posteriorem valde approximato, apertura ovato-elliptica; long. 5, lat. 4 , alt. $21 / 9-3 \mathrm{~mm}$. - Norv. at Kristiania, Asker, Sogneselven, Fiskum et Slkien, vulgatior quam typus. (B. Esm.).

A. danicus n. sp. T. tenuissima, alba, nitida, perdepressa, elongato-elliptica, forma (et tenuitate) A. (Velletia) lacustri L. persimilis, densissime concentrice striata (striis nune for' liorıbus nunc tenuioribus), striis radiantibus obsoletissimis ad margines plano-dilatata, apice obtusissimo, oblicquo, excentrico, margini dextro forte approximato, supra compresso, post apicem recte declivis; apertura elongato-elliptica, postice angustior. Long. $6^{1 / 2}-8$, lat. $3-4$, alt. 2 mm. IIab. Dania ad Aarhus in penins. Jylland. (H. Sell).

2. Velletia Gray. T. dextralis, sentiformis, membranacca, non nitida, apice acuto ad sinistrum.

A. Iacustris Lin. (Syst. nat., 1758, Patella). T. fuscescens, cornea; apertura oblonga, extus paullo dilatata. Long. $6-8$, lat. $3-3^{1} / 2$, alt. $2-3 \mathrm{~mm}$.

Hab. Suecia merid. et media sparsim usque in Dalarne. - Norv. meridionalis. - Dania vulgaris. - Fennia meridionalis (ut in Ảland ad Eckerö, ad Frugård, Rautus et Satakunta). 


\section{Ordo 2. Opereulata.}

Testa spivalis operculata (operculum supra pedem animalis postice aunatum).

\section{Subordo 1. Geophila.}

II. pulmonate, terrestria.

\section{Fam. Cyclostomidx.}

Testa ovato-conica vel cylindrica, operculo spirali (spiris paucis rapide accrescentibus).

Subfam. Truncatellina.

Testa cylindrica, obtusa, nitidissima, apertura oialis. sunerne acute angulata, marginibus subparallelis, operculum corneum, tenuissimum, hyalinum.

\section{Gen. Acme Hartm.}

A. polita Hartm. (Gasterop. Schweiz, 1840). T. fuscobrunnea, lavis, polita; anfr. 5-6; apert. intus forte labiata, extus callo purpurascente sat valido cincta. Long. 3---4, lat. ${ }^{1 / 2}$ $-1 \mathrm{~mm}$.

Hab. Suecia in Skåne (in prëuptis sehistaceis ad Marielund prope Ringsjön). — Dania in Sjælland (Kastrup Fredskor ad Ringsted) et Fyen (ad Odense Fjord: H. L.).

A. lineata Drap. (Tabl. Moll,, 1801). T. corneo-fusca, lineis longitudinalibus numerosis impressis, distantibus sculpta; anfr. $6-7$; apert. labiata, extus callo rufo cincta. Long. $3^{1 / 5-}$ i, lat. $1 \frac{1}{8}-1^{1 / 4} \mathrm{~mm}$.

Hab. Dania in ins. Fyen sex annotatione a cl. Möller, determinatio tamen non omnino certa»: O. Mörch); ad Odense prope sinum, unde specimen optimum in collectione am. H. Lỵnge vidi.

\section{Subfam. Cyclostomina.}

Testa ovato-turvita, acuminata, clathrata, apertura (ovata) rotumlata, peristomate continno, operculum calcureum. crassum. 


\section{Gen. Cyclostoma Lam.}

C. elegans Mïll. (Verm. Hist. II, 1774, Nerita). T. extus cinereo-cornea, intus fulvida, sæpe minutim obscure variegata; anfr. 5, convexi. Alt. 13-14, lat. $81 / 2-9 \mathrm{~mm}$.

Hab. Dania in Sjselland multis locis frequens v. frequentissima, in ins. Flatö (A. B. \& J. C.) et Bogö (H. L.) juxta Falster, nee non in Jylland.

\section{Subordo Hydrophila.}

11. branchicata, aquatitia.

\section{Fam. Paludinidre.}

Testa magna, ovato-conoidea, epidermide olivaceo, scepe fasciis longitudinalibus tribus fuscolnumneis et lineis spiralibus subtilissimis ornata, anfr, ult. ventricosus, apert. rotundato-ovuta, obtuse angulata, operculum comeum, concentrice striatum, parum immersum, nucleo laterali.

\section{Gen. Paludina Lam.}

P. contecta Mill. (Noll. Maine et Loire, 1813, Cyclostoma). T. perforato-umbilicata, globoso-conoidea, tenuis, spira apice acuto prominente (plerumque eroso), celeriter angustata; anfr. (6) 7, inflato-convexi, eylindracei, penultimus superne subplane-transversus, infra constrictus, ultim. ampliato-tumidus, sutura profundissima, canaliculata. Alt. 40, lat. 28 -30, ap. $\lg .18 \mathrm{~mm}$. (ex. max.). Testa juvenis granulata, anfr. ult. brevissime hispido et costis spiralibus tenuibus tribus, ciliis rufis recurvis munitis, ornato. - Cycl. vivipara Drp. (1805), Pal. listeri Forb. \& Hanl. (185̈3).

Hab. Suecia in Skåne multis locis, Blekinge passim, Smàland (tantum in parte merid. gub. Calmariensis) et $\mathbb{W}^{\top}$ estergötland (circa Göteborg) h. e. orientem versus ad $56^{\circ}$ et occidentem versus ad $57^{2} / 3^{\circ}$ lat. bor. - Dania in Sjæelland, Lolland et Jylland, nec non (fide H. Lynge) in Möen et Falster. - Fennia in Carelia ad Kivinebb in Sinu fennico, ad Gorki prope 
flumen Svir, in Ladoga ad Valamo, in Yskjärvi, Schlüsselburg etc. (Mus. Helsingf.).

Var. lacustris Beck (Amtl. Ber., 18. 7 ): t. ventroso-elongata, olivacea, leviter fasciata, regulariter striatula, fortius ad aperturam, anfr. 7 , teretes, apert. vix obliqua, ovalis, peristomate fere continuo; alt. 4-í, lat. 33, ap. lg. 20, lat. 17 mm. - Dania notamment lans le canal de Stovozze (Bourg.:).

Var. paludosa Bourg. (Récens. Vivip., 1880): t. vix rimata, elongata, brunnea, concolor, spira obtusa, apice tenui, anfr. 6, convexi, rotundati, celerrime accrescentes, sutura superne tenuis, infra profunda, apertura perobliqua, marginibus tenue junctis, margine columellari valido, crassissimo; alt. 35, lat. 24, ap. lg. 17, lat. $15 \mathrm{~mm}$.-- Dania dans les marais (Bourg.).

P. gallandi Bourg. var. occidentalis Bourg. l. c.: t. rimata, ventroso-elongata, olivacea, obsolete trifasciata, spira conoidea, rotundato-obtusa, apice non prominente at tenui, anfr. 3. et 4. subrequales, apert. oblonga, margine columellari solido, incrassato. Alt. 20, lat. 16, ap. lg. 12, lat. $9 \mathrm{~mm}$.

Obs. Hab. Dania (Bourg.). Formas tres ultimas e Dania beat. O. Mörch ad beat, R. J. Bourguignat misit et auctor meritissimus gallicus ut species descripsit. Locus specialis danicus V. lacustris, lapsu calami vel interpretatione falsa tristis picturæ communicatoris, non recte a Bourguignat allatus, est in sinu Lymensi ad ostium canalis Storvorrecanal dictæ (Bk.)» ut scripsit Mörch ipse in Syn. Moll. Danif. P. occidentalis ad gregem P. gallandi Bourg. (cujus typus ad Constantinopolin occurrit) pertinet et rivit etiam in Gallia et Britannia.

P. vivipara Lin. (Syst. Nat. X, 1758, Helix). T. imperforata v. obtecte rimata, ovata, solicka, pallida, virescens, distincte trifasciata, spira conica, obtusa, quam apertura paullo longior, apice tenui prominente; anfr. $5^{1} / 2-6$, convexi, lentius et regulariter accrescentes, sutura superne parum, infra sat profunda, ultimus convexus. Alt. 25-28, lat. 18 -20 (ex. max. 37-26) mm. - Nerita fasciata Mï̈ll. (1774) p. p. $=$ P. vivipara L. \& P. pyramidalis Jan!, Cycl. achatinum Drp. (1801).

Destributio hujus speciei omnino alia quam precedentis in Suecia est, limitem meridionalem suum mare balticum versus 
longe a limite boreali illius habens. Terminus borealis ultimus cognitus est ad ostium fluminis Dalelfven $\left(60^{\circ} 30^{\prime}\right.$ lat. bor.) et terminus meridionalis paullo infra $58^{\circ}$ (ad Westervik). Occurrit ergo in provinciis Östergötland, Nerike, Södermanland, Upland et Westmanland (locus orig. Linnæi »in fluvio Sahla» pone $60^{\circ}$ lat. bor.), fere ubique copiose, vel in regione orientali-boreali Querci roburis, dum P. contecta regionem Fagi sylvatica occupat. - Dania solummodo in Jylland. - Fennia ad Schlüsselburg (Mus. Helsingf.) et (fide $\mathrm{W}$. L.) in Ladoga.

Obs. Huc certe P. viripara Friele in Norske Mollusker 18503 pertinet (quod e descriptione, magnitudine - long. 20-25 $\mathrm{mm}$. - et loco ad $60^{\circ}$ lat. bor. apparet, quanquam ut auctor Lamarck citatus est: "in lacubus et paludibus passim circa Kristiania", ubi postea numquam reperta est (fide B. Esmark).

Var. nexilli Bourg. (Recens. Vivip., 1880) aff:: t. obeso-ovata,rentricosior, fusco-olivacea, nigricans, fasciis tantummodo intus distinctis, spira brevior (long. aperturæ, crassior. perobtusa), anfi. $4 \frac{1}{2}$, primi depressi, ultimi quam in typo majores, altiores, ultimus ventricosior, sụtura minus impressa, apert. fere æque lata ac longa, margine exteriore lerius, colnmellari fortius arcuatis; alt. $25-30$, lat. $20-23 \mathrm{~mm}$. - Suecia ad Sundbyberg prope Stockholm $(\mathrm{Hj}$. W.). Specimina juniora (,a. 23, l. 17 mm." B.) aperturam paullo quam spira longiorem habent.

\section{Fam. Bythiniida.}

Testa minor, ocato-conica, non fasciata, spira conica, anfi. unt. ventricosus, apert. rotundato-ovata, obtuse angulata, operculum corneo-culcareum, concentrice striatum (i. in centro spirale), terminale, mucleo subcentrali.

\section{Gen. Bythinia Leach.}

Obs. Rerisio nora hujus generis docuit synonymiam nostrarm formarum ralde confusam esse et me cogitarit omnino ad sententia. meas pristinas, in Fauna moll. Suecire 1873 p. $461-465$ expositas, redire.

B. tentaculata Lin. (Syst. Nat. X, 1758, Helix). 'T. imperforata v. angustissime rimata, sub lente densissime spiraliter lineata; anfr. plano-convexi; sutura linearis v. parum im- 
pressa, tandem lente longeque descendens; peristoma continuum affixum; operculum (ut apertura) ovatum, superne angulatum.

Typus: t. ventroso-conoidea, sat tentris, nitida, flavido-cornea, anfr. 5-6, superi 3-4 regulariter in spiram brevem late conicam attennati, ultimi duo majores, plus quam $2{ }_{3}$ altitudinis totius occupantes, ult. ventroso-convexus, apert. subovalis, margine colum. fortins incurvato, opercultum lineis concentricis tantum 1-2 elevatis, ceteris tenuissimis; alt. 8-10, lat. 5 -6 mm. - Nerita jacnlator Mä̈ll. (1774), Palud. impura Drap. (1805).

Forma gigas Mörch (Syn. Moll. Dan., 186\%), t. rimata, late orata, anf. ult. lineis transversis 2-3 elevatis, apice eroso et lamina plana suppleto; alt. 1j, lat. 9, ap. a. 7, 1. $5 \mathrm{~mm}$. - Suecia in Skàne (Höjeả). - Dania in Viborg Sö. - Fennia in Alland (in palude ad Godby: Mrus. Helsingf.).

Forma intricata W. (Nachr. blatt Mal. Ges. 18!)'́), t. minor, operculum lineis incrementi concentricis omnibus valde elevatis, interstitiis densissime striatnlis. - Suecia ad Borgholm frequens.

Forma gemina W. (Nachr. blatt Mal. Ges. 1894), t. operenlo extus spatio lato lævi vel striatulo, lineis incrementi concentricis $3-5$ internis furte elevatis.

- Suec. ad Ultuna prope Upsala frequens.

Var. producta IIke. (Syn. ed. 2, 1830): t. major, elongato-conica, solidior, cornea, anfr. 6-7, spira elongata quam apert. longior, acuta, anfir, ult. elongatus, parum convexus, apert. angristior, acutior; a. 12--15, lat. $7-8(18: 10) \mathrm{mm}$. - Suecia in Skåne et Östergötland inventa.

Var. ventricosa Mke. (Syn. ed. 2, 1830): t. vix rimata, ventrosa, tenuis at firma, cornea. nitidula. anfr. $\check{5}$, sat celeriter accrescentes, convexi, numc perdistincte, nunc obsolete superne ad suturam sat impressam planati et angulati, ult. ventrosus, snperi 3 sat abrupte in spiram brevem anguste conicam attenuati, apert. quam spira panllo brevior, rotumtato-ovata, marginibus æeque arenatis; alt. 10, lat. $7 \mathrm{~mm}$. - Snecia ad Stockholm (Nya Brumsviken: J. W.). - Fennia in Ladoga (Mus. Helsingf.). „B. inflata Hansén, telle que je l'ai compris dans ma monographie, avec Bourguignat" (Locard in lit.).

Var. bottnica (Anders.) Cl. (Malak. Blätt. 1878): t. firma, flavovirescens vel fulva, forte spiraliter lineata, rugis incre- 
menti 1-2 atris, intus aurantiaca, anfir. 5, convexi, celeriter accrescentes, apertura atromarginata, albolabiata; alt. 13-1. f, lat. $8 \mathrm{~mm}$. - Suecia in sinubus maris prov. Medelpad.

Var. sebethina (Blanc) Cout. (Ann. Soc. Linn. Lyon 1881): t. elongata, spira longe lenteque attenuata, acute conica, anfractus convexi, ult. elongatus, parum ventrosus, sutura profundior, apertura quam spira brevior, elongato-orata, acute angulata, operculum in centro distincte spiraliter contorta; alt. $9-12 \frac{1}{2}$, lat. $6-7 \mathrm{~mm}$. - Suecia in Öland (ad Borgholm).

Var. michaudi Dural (Rev. zool. soc. Cuvier 1845): t. non rimata, ovato-conica, rufescenti-cornea, spira attenuato-conica, quam apertura (interdum multo) longiore, anfr. 5-6, convexi, celeriter accrescentes (quisque spiræ fere duplo altior quam præcedens), ultimus tumido-convexus, sutura profunda, apert. late ovata; a. $11-13,1.5-7 \mathrm{~mm}$. - Suecia ad Ronneby (in lacubus Herrstorpsjön et Tresjön), Stockholm (in Djurgården: J. \& Hj. W.), Upsala (ad UItuna: IV. L.).

Forma (rel monstrositas) excavata Jeffr. (Brit. Conch. 1. 1862), t. magna, anfi. valde convexi, sutura excavata. - Suecia ad Lund, Ronneby (in Herrstorpsjön), Upsala (ad Ultuna), Gotland (ad Lummelund). - Dania in Bornholm.

Var. decipiens Millet (Rer. \& Mag. Zool, 1843): t. anfractu ultimo magno, tumido-convexo, ceteris convexiusculis, $3{ }_{1}^{1}$, superis spiram brevem anguste conicam acutamque formantibus, sutura impressiuscula, antice fortius descendens. apert. quam spira brevior, ovata, margine exteriore fortius arcuato; a. 10, 1. $6 \mathrm{~mm}$. - Suecia in Skåne (ad Belteberga et Kristianstad), Dalarne (in Brunnsjön), Gefle.

Forma opportuna mh., t. anfr. 4 superis convexis, angustioribus et spiram altiorem acutam formantibus. - Suecia ar Istad in portu. Tenet medium inter decipientem et sebethinam.

Var. soluta mh.: t. rimata, ovato-conoidea, rufescens, spira producta, conica, acuta, anfr. $5^{1} / 2$, superi convexiusculi, sutura tenui, penult. convexus, sutura antice profunda, ultimus parum major, tumido-rotundatus, apert. subverticalis, soluta, orata, superne subacute angulata, opereul. striis concentricis marginalibus crassis; a. 11, 1. 5, apert. 1. $1 \mathrm{~mm}$. - Suecia in Gotland (ad Lummelund: G. E.).

Nerita sphorica Müll. (Verm. Hist. 1774) : ,t. subglobosa, diaphama, 
alba v. cinerea, anfr. 4, convexi, infimus ventricosus, reliquis ommibus simul sumtis duplo longior et largior, cum in jaculatore (tentaculata L.) longitudine æqualis sit; diam. ${ }^{2 / 3}-2$ lin. In paludosis (Daniæ) minus frequens." Juvenis?

Hab. Typus: Suecia a prov. Skåne usque in prov. Medelpad. Norv ad Kristiania. - Dania tota. - Fennia meridionalis et media.

B. leachi Shepp. (Trans. Linn. Soc. 1823, Turbo). T. late rimata vel subperforata, sub microscopo densissime spiraliter lineata; anfr. inflati, subcylindracei, sutura profunda v. profundissima, antice subhorizontalis; peristoma continuum vel subsolutum; operculum (ut apert.) rotundatum, superne non vel obsoletissime angulatum.

Typus: t. late rimata, orato-conica, basi sat ventricosa, lutescenti-cornea, spira breviter conica, acuta, subgradata, ex anfractu ultimo ad sinistrum dilatato subite attenuata, anfr. 5 , forte convexi, teretes, subscalarati, superi intira subito constricti, sutura inferne profundissima, canaliculata, penultimus major, superos altitudine æquans, ult. rotundatus. quam spira brevior, apert. ovato-rotundata; a. $5-7,1.4-5$ mm. - B. ventricosa Gray 1821 (nomen!), B. sp. Nilss. 1822, B. troscheli Paasch 1843, B. inflata v. minor Hansén 1845 , B. similis pr: p. Stein 1850, MaIm 1855, B. Ieachi Jeffr. 1862, Reeve 1863, B. ventricosa $\nabla$. minor Mörch 1864. B. inflata Cl. 1837 (exl, syn, omn.).

Var. inflata Hansén (Ötrers. K. Vet. Ak. Förh. 1845): t. pertorato-rimata, subovata, ventricosa, tenuiuscula, pallide cornea, spira brevis; late conica, anfr. 5, valde et regulariter convexi, sutura profunda, ult. inflatus, ad sinistrum late convexus, spiram subæquans, apert. subovato-rotundata; a. $8-11,1.5-7^{3} / 4$ nmm. -- Suecia ad Norkköping (in Lillån). - Dania ad Kjöbenhavn (Nörreport). - B. similis Stein pr. p. 1850, B. inflata Küst. 1852, B. ventricosa Mörch 1864. B. transsilvanica Bielz olim, B. troscheli Bielz 1867.

Hab. Typus: Suecia in Skảne, Öland, Gotland, Östergötland, Westergötland, ad Stockholm (Djurgårdsbrunnsviken, Husarviken, Djursholm etc.) Upland (ad Upsala). — Dania in Sjælland, Fyen, Möen et Jylland. 


\section{Fam. Rissoidre.}

Subfam. Hydrobiina.

Testa minuta; operculum coriaceum v. comenm, spirale, spiris paucissimis $\left(1-2^{1}\right)$, rapide accrescentibus, profunde immersum, mucleo valde excentrico.

\section{Gien. Hydrobia llartm.}

H. steini Mart. (Arch. f. Naturges. 1858). T. rimato-subperforata, turrito-conica, cylindracea, tenuissime striatula, corneo-virescens, spira obtuso-subtruncata, longior quam apertura; anfr. 5, sat lente regulariter accrescentes, convexi, sutura canaliculata, ultimus convexo-rotundatus; apertura ovalis, superne obtusissime angulata. peristomate connexo. Alt. $3^{1 / 3}-3^{1} / 2$, lat. $1^{5} / 6-2 \mathrm{~mm}$.

Hal. Suecia in Skåne (Wesum pr. Lund, Kristianstad), Blekinge (Bastasjö \&c.), Småland (Jönköping), Gotland (Gothem), Östergötland (Kimstad et in palude ad Ljungstorp), Westergötland (Kopparsjön pr. Mölltorp, Göteborg), Nerike (Svartån pr. Hjelmaren), Upland (Upsala). — Dania ad Kjöbenhavn, Esromsö et Vorösö (H. L.).

Var. scholtzi A. Schm. (Bimenmoll. Norddeutschl. 1856): t. ovato-conica, spira vix longior quam apert., late conica, anfi. 4, altitudine lente, latitudine celeriter accrescentes, ult. ventrosus, ad sinistrum dilatatus; alt. $2-2^{3 / 4}$. lat $1 \frac{1}{2}$ -2 mm. - Suecia ad Romneby (in lacubus Herrstorpsjön \& Långasjö). - Finland ad Frugård (E. N.).

\section{Fam. Valvatida.}

Testa discoidea usque ad conoidea, apertura subcircularis, marginibus continuis; operculum multi- et arctespirale, nucleo centrali, mofunde immersum.

\section{Gen. Valvata Müll.}

1. Cincinna Hübn. T. turbinoidea, umbilico angusto, scepins ad partem obtecto.

V. antiqua Sow. (Mlag. of Nat. Hist., 1838). T. anguste, sæpe obtecte, perforata, ovato-conoidea, dense striatula, sape 
spiraliter lineata, calcarea (viva viridula), spira alta, acuminato-conica, (fnam apert. sxpius nulto longior; anfr. 5 , lente regulariter accrescentes, subteretes, ultimus siepe solutus; sutura impressa; apert. rotundata. Alt. 5-6, lat. 4. $\AA^{1} / 2 \mathrm{~mm}$.

Haל. Succia in maxime orientali parte prov. Skåne (Râbelöf, Ölsjö, Krageholm, Lyngby et Lefrasjön), in Östergötland (copiose in lacu Tâkern) et Upland. In lacubus Lefrasjön et Takern viva inventa. - Dania in plerisque lacubus majoribus.

V. pusilla Müll. (Verm. Hist. II, 1774, Nerita). T. umbilico perspectivo cylindrico, globosa v. pomacea, corneo-subviridis, striis incrementi obsoletis, spira sat elevata, apice planiusculo; anfr. 4. Lat. $3^{1} / 2 \mathrm{~mm}$. Neritinam piscinalem valde refert, at multo minor (diam. 1 lin.) et tamen anfractuum quattuor est» (Müll.) Hiec forma quam maxime V. antiquie similis, sed differt in speciminibus acque magnis umbilico cylindrico perspectivo: (Mörch).

$H a b$. Suecic in Östergötland (in lacu Tảkem), ad Stenborg prope Gefle et ad Jörn in Westerbotten. - Dania in Sjielland (Damhuussöen et in pluris locis, fide Mörch, qui hue refert V. piscinalem Nord. \& Nyl. (Finl. Moll. t. IV f. 56). - Fennia (Borgå, Taipalsaari et Paanajürvi in Kuusamo). Hice forma mihi nondum clara est.

V. lilljeborgi n. sp. 'T. rimato-perforata, subovata, dense striata; anfr. $4-4^{1} / 2$ convexi, cylindracei, non gradati, sutura parum profunda, penultimus antepenultimo plus quam duplo altior, ultimo non ventroso, nee dilatato, parum prievalente 1/4 humilior; spira ovato-conoidea, obtusa, infra latitudine altitudini subæcualis; apert. subcircularis parum obliqua, peristomate sub mediun anfr. penultimi longe abbreviato v. callo affixo tenui conjuncto. Alt. $\{-5$, lat. f $\mathrm{mm}$.

Huð. Suecia in fluvio Eyrisan ad Upsala (IV. L.).

V. piscinalis Müll. (Verm. Hlist. II, 1774). T. anguste, sapins panllo obtecte umbilicata, oblique globoso-turbinata, striatula, fuscocornea, rufa v. virescens, spira altitudine aper- 
turæ, obtusa, apice depresso; anfr. 4-41/2, sat celeriter accrescentes, rotundati, ultimi duo forte dilatati, ultimus penultimo multo major, ventrosulus, superne depressus, sutura nediocri; apert. rotundata, ad dimidium sub anfr. penultimum, margine columellari ad $1 / 3$ adnato. Alt. 5, lat. $4^{1} / 2 \mathrm{~mm}$.

Hab. Suecia a Skåne sparsim usque in Jemtland (Storsjön et Ragundaelf) et Pite Lappmark. - Norv. meridionalis et media. - Dania sat vulgaris. - Fennia tota usque in Lappland (Sodankylä in fluvio Iesiö.)

Var. borealis Milach. (Etudes moll. Moscou, 18s1): t. aperte umbilicata, turbinata, tenuis, corneo-rirescens, anfr. 4, teretes, sutura profunda discreti. temue striata, lineis spiralibus obsoletis, apert. aperta, circularis; alt. \& lat. $4 \mathrm{~mm}$. - Suecia in Jemtland (Krokom, Kall, Östersund \&c.: E. N.). - Norr. ad Tromsö (E. N.). Ostfinmarken ad Koskiniari, Wagattemjauri, Tschalmajauri, Wardukoski ete. (Ch. Ra. bot). - Femnia lappon. ad Kralajokki et Kamasjokki (Ch. R.).

Var. discors IV. (Fauna Binnenconch. pal. Reg. TI, 1886): t. ovato-conica, apice rotundato-obtuso, forte striata r. subcostata, anfr. 4, ultimi duo subrquales, sutura profunda, apert. parra, ad $2 / 3$ sub anfr. peunltimum, operculum lineis spiralibus forte elevatis, umbilicus angustus, semiobtectus; lat. 3, alt. $3 \mathrm{~mm}$. - Suecia in Skåne (Ringsjön). Var. costulata W. in Exk. Fauna, nec Dronët.

Tar, ambiqua W. (Fauna Suec., 1873): t. umbilico conico-perspectiro $\left(1-1 \frac{1}{2} \mathrm{~mm}\right.$. lato), conoideo-depressa, pallide rirescens, subtiliter et dense at regulariter et argute striata, anfi. $3 \frac{1}{2}-4$, ultimi celeriter accrescentes, ultimus magnus. ventrosus, antice latitudine et altitudine æqualis, apert. magna, tantum ad $1 / 3$ sub anfr. penult., operculum lineis spiralibus argutis; lat. 4--5, alt. $31 / 2-1$ mm. - Suecia ad Göteborg*, ad Romneby, in Dalarne (ad Tåbäck) et in Jemtland (ad Lockne: E. N.). - Fennia in Kuopio (in amne ad Jynkkä: K. M. L.).

V. cyclomphala $\mathrm{W}$. (Comptes rend. d. séances de l'Ac. des sc., Paris, 24 juin 1889). T. sat late et perspective umbilicata (umbilicus circulari-infundibuliformis), depresso-conoidea, prasina, laevis v. vix striatula; anfr. 4, convexi, suturam profundam subcanaliculatam versus constricti, ulti- 
mus cylindraceus, penultimo vix $1 / 3$ latior; apert. circulalis, anfr. penult. breve adnata. Lat. $4^{1} / 3$, alt. $2^{3} / 4 \mathrm{~mm}$. Hab. Norvegia in Ostfinmarken ad Koskiniavi (69 lat. bor.) prope flumen Pasvig (Ch. R.).

Forma halopea $\mathrm{mh}$., t. argute dense striata, apert. anfractum penultimum vix tangit; lat. 4 , alt. $3 \mathrm{~mm}$. - Fennia ad lacum Kallavesi in Kuopio.

V. alpestris (Blauner) Küst. (Chemn. Conch. Cab., 1852). T. aperte umbilicata (umbilicus intus perangustus, ad aperturam celeriter dilatatus), depresso-turbinata, regulariter tenue striata, virescens, corneoflavida v. cinerea, spira depressa, lata, obtusa; anfr. 4-41/2, celeriter accrescentes, teretes, ult. ad suturam profundam impressus, antice sæpe solutus; apert. spatio minutissino adnata v. soluta. Lat. 4-5, alt. $3-4 \mathrm{~mm}$.

Hab. Suecia vivens et frequens in Jemtland (ad Östersund: E. N. \& E. C.) et Pite Lappmark (ad Jörn: E. H.); subfossilis in Öland (in paludibus turfosis ad Borgholm) et Gotland (Likmäde ad Hemsö: E. N.). -- Femia vivens in Kallavesi prope Säyneensalo ad Kuopio (Mus. Helsingf.).

†V. glacialis W. (K. Vet. Ak. Förh., 1881). T. umbilicata (umbilicus intus cylindricus, a medio anfr. penultimi forte dilatafus, perspectivus), ovato-conoidea, calcarea, regulariter pulchre striata, spira obtusa quam apertura altior; anfr. 5 , perconvexi, sutura profundissima, ultimus plus minusve et sxpe longe solutus; apert. subcircularis v. superne obsolete angulatus. Lat. $4 \frac{1}{2}$, alt. $5 \mathrm{~mm}$. Testa sicpe scalaris, anfractubus omnibus disjunctis.

Hab. Suecia in Skåne (subfossilis in palude turfoso ad Stenstorp).

V. raboti W. (Comptes \&c. Paris, 24 juin 1889). T. minima, anguste, ad partem obtecte umbilicata, globoso-depressa, utrinque dense costulata, circa umbilicum concentrice striata; anfr. $3^{1} / 2$, convexiusculi, celeriter accrescentes, ultimus magnus, antice celeriter et forte dilatatus, rotundatus; apert. magna, circularis. Lat. $1 \frac{2}{3}$, alt. $1 \mathrm{~mm}$. 
Hab. Norvegia in Ostfinmarken ad Klistervand, $69^{\circ}$ lat. bor. (Ch. R.).

2. Tropidina Adams. T. depressa, spira mominula, subtus scepius late umbilicata.

V. nana W. (Fauna Suec., 1873). T. minima, sal anguste profunde umbilicata (umbilico subcylindraceo), subdiscoidea, spira tamen distincte supra anfractum ultimum elevata, brunnea, striata; anfr. 3-3 $3 \frac{1}{2}$, sat celeriter accrescentes, ultimus eylindraceus, aperturam versus utrinque distincte dilatatus; apert. circularis, magna, spatio lato ad anfr. penultimum affixo. Lat. $1-1^{1 / 2}$, alt. $1 / 2-^{3} / 4 \mathrm{~mm}$.

Hal. Damia. Locus originalis ignotus. - V. minuta Mörch \&. W. olim, nec Fér.

V. macrostoma Steenb. (Amtl. Ber., 1847). T. sat late et perspective umbilicata (umbilicus ad apert. celeriter dilatatus), spira convexiuscula, prominula (ad dimidiam altitud. aperturio), firma, cornea, nitidula, distincte et argute striata; anfr. $3 \frac{1}{2}$, sat celeriter accrescentes, forte convexi, ultimus subteres, subtus aperturam versus dilatatus; apert. magna, c. $1 / 3$ sub anfr. penultimum, brevissime adnata. Lat. $3^{1 / 2}$ -4 , alt. $2 \mathrm{~mm}$. (major l. $5-6$, a. $2 \frac{1}{2} \mathrm{~mm}$.).

Hal. Suecia in Skîne (Kristianstad), Östergölland (in multis locis) et Jemtland (ad Östersund: E. N.). - Norv ad Stavanger. - Dania ad Kjöbenhavn et Slagelse. - Femia multis locis (Nikola ad fl. Svir, Kenjärvi, Vosnesenje, ad Tetrina et Varsuga in Lapponia rossica, etc.: Mus. Helsingf.).

Forma mallcata W. (Exposé crit., 1871), t. lineis spiralibus elevatis, interstitiis malleatis. - Dania.

Var. umbilicata (Fitz., nomen) W. (Fama Binnenconch. pal. Reg. VI, 1886): t. umbilico magis et celerins dilatato, soldide corneo-flavida, striatula, spira fere plana, anfi. celerius accrescentibus, ultimo subtus paullo planulato, apert. fere omnino extra anfr. penultimum; lat. 5, alt. 2 mm. Snecia in Upland (ad Ultuna) et Gotland. - Dania ad Rude, Sorösö et Viborgsö. - V. spirorbis Küst. (nec Drap.), V. frigida Cl. (nec W.).

V. pulchella (Stud., nomen) W. l. c.: t. angustius umbilicata, subturbinata, substriatula, cornea, spira altior, depresse 
conica (fere altitudine aperture), truncata, anfr. 4 , sat lente accrescentes, subscalares, ultimus subtus antice dilatatus, apert. paullisper sub anfi. penultimum; lat. 1-5, alt. 3--4. mm. - Suecia in Skåne (ad Ousbyholnn). - Fennia in Saimen. - V. depressa Kob., Cl. \&e.

V. frigida WT. (Fauna Suec, 1873). T. discoideo-depressa, subtus late umbilicata, dense et argute striata, olivaceo. $v$. virescenti- colnea v. argenteo micans; anfr. 4, superi sensim, cateri celeriter accrescentes, subcylindracei, ultimus antice dilatatus, sapius humilior quam penultimus; apert. magna, circularis, ad $1 / 3$ sub anfr. penultimum et sat late adnata. Lat. 5, alt. $2 \mathrm{~mm}$.

Hab. Suecia in Pite Lappmark (ad Naustejaur:" - Fennic in Enare Lappmark (ad Joinkoski in fluvio Muonio ad templum Muonioniska).

3. Gyrorbis Fitz. 1833. T. discoidea, spira plana, subtus late umbilicata 2 . concara.

V. cristata Müll. (Verm. Hist. II, 1774). T. tenuissime striatula, brunneo-fulvida v. cinerascens, nitida; anfr. $3^{1} / 2-4$, subteretes, sat lente accrescentes, ad suturam profundam canaliculatam obtuse angulati et declivi, omnes subtus fere reque distincti ac supra; apert. circularis, omnino extra anfr. penultimum. Lat. $2-3$, alt. $1-1^{1 / 2} \mathrm{~mm}$.

Hab. Succic in Skåne et Halland passim frequens, in B]ekinge rarissime (in lacu Wesan et ad Ronneby $1 \mathrm{ex}$. inventum), in Oland, Gotland et Östergötland passim frequenter, Westergötland (ad Göteborg fere rulgaris), Nerike (in tota regione campestri vulgatissime, in regione silvestri passim), ad Stockholm et in Upland passim, in Westmanland vulgaris, in W'ermland (in lacu Wenern par. Botilsssäter) et Jemtland (ad Lockne: E. N.). Teste S. Nilsson (in Hist. Moll. Su.) usque in Lapponiam hre species ascendit, si non confusio cum V. frigida exstat.Norv. meridionalis. - Dania vulgaris. - Fennia in Åland, Geta Emkarby, ad Luhanko et in Ytterön ad Björneborg (Mus. Helsingf.). 


\section{Fam. Neritinida.}

Testa scepins crassa, semiglobosa (supra convexa, subtus plana), transversalis, columella plana, latissima, declivis, margine acuto, spira brenissima, excentrica, operculum (bispirale), articulatum calcareum, firmum.

\section{Gen. Neritina Lam.}

N. fluviatilis Lin. (Syst. Nat. X, 1758, Nerita). T. oblonga, pallide virescens (violascens v flavescens), striatula, lineis et maculis fuscis reticulata, sæpe fasciis tribus fuscis obsoletis ornata, spira brevi, depressa; apert. semicircularis, intus pallide virescens, plaga columellari pallida; operculum crocaceum, margine miniato. Long. transv. 10-12, alt. apert. $7-8 \mathrm{~mm}$.

Hab. Suecia, in provinciis litoralibus orientalibus saltem usque ad ostium fluvii Dalelfven, formæ fluviatiles et lacustres præcipue in prov. Skåne, ceteroquin forma baltica ubique prævalens vel sola, sed nulla forma in provinciis internis inventa. - Norv. passim ad ostia fluviorum et rivorum (fide H. Friele). - Dania in una alterave forma vulgaris, typica rara excepta. - Fennia tantummodo forma baltica.

Var. fontinalis Brard (Coq. de Paris, 1815): t. tenuis, pellucens, fusca, albomaculata. - Forma rivalis.

Var. lacustris Lin. Fanna Snec. ed. 2, 1761, Nerita): t. tenuiuscula, coerulescens $\mathrm{v}$. pallide virescens, variis modis picta, intus cum plaga columellari crocea $\mathrm{v}$. citrina, spira elata; long. transv. 9-12 mm. - Suecia in Skåne multis locis. - Dania in lacubus et in rivis ad lacus.

Var. spirate W. (Fauna Suec., 1873): t. crassissima, calcarea, albida, unicolor $v$. lineis fuscis reticulata, antice interdum rufostrigata et costulis transversalibus irregularibus, intus crocea, operculo et plaga colum. subviolaceis v. flavescentibus, opercul., margine sæpe atro, spira alta, elata. anfr. penult convexus, apertura marginibus sepe callo crasso junctis; long. transv. 11, alt. $9 \mathrm{~mm}$. (spec. max). - Suecia in Gotland (in palude ad Fardume " et ad Ar frequens).

Forma minor, long. tr. 7 , alt. $5 \mathrm{~mm}$. - Suecia in Gotland cum typo, Upland ad Ultuna. 
Acta Societatis pro Fauna et Flora Fennica, XIII, n:o 7.

Var. litoralis Lin. (Syst. Nat. X, 1758, Nerita): t. sat tenuis, nigrescens $v$. fusca, subunicolor v. albomaculata; long. tr. 8-10 mm. - Ad litora marina fere ubique Sueciæ, Daniæ et Femniæ intra limites allatas.

Forma baltice Beck ap. Örsted (De Regionib. veget. in Öresund, 1842), t. minor, nigrescens, unicolor v. obsolete variegata, intus cum plaga colum. pallide coerulescens, spira brevissima, depressa; long. tr. $5^{1 / 2}-7 \mathrm{~mm}$. - Suecia passim. - Dania in Sjælland (Kallebrostrand, portus Bisserup ad Skjelskör, ad Tre kronor \&c.).

\section{Cl. II. Malacozoa Acephala.}

Mollusca aquatilia hermaphrodita, capite mullo, branchiis quattuor marnis foliaceis respirantia, testa bivalvi (concha) apud nos semper cequivalvi tecta.

\section{Fam. Sphariida.}

11. pallio antice aperto, postice connato, in tubum geminem vel simplicem contractitem extenso. Concha parva. globosa, ovalis vel subtriangularis, dentibus cardin. 2-3, lateralibus iamelliformibus.

\section{Gien. Sphærium Scop.}

C. subrequilateralis, ligamento parte postica paullo longiore alnato. Animal siphonibus duobus longis, tantum basi comnatis.

(Concha mullarum quo junior eo magis compressa, quod observes ne illam pro Pisidium capius).

1. Spheriastrum Bourg. C. firma, costulata, umbonibus latis, rotundatis, ligamento externo valido, dentilus carlin. valvulce utrinsque dubus juxta positis.

S. rivicola Leach. ap. Lam. (Hist. anim. s. vert. V, 1818, Gyclas). C. ovalis, ventrosula, dense arcuatim costulatostriata, corneo-fusca, intus coerulea; umbones depressi. Long. 19-23, alt. 14-15, crass. T-9 $\mathrm{mm}$.

Hab. Dinie in Jylland (Hanherred). 
1.4́ C. A. Westerlund, Synopsis Molluscorum Extramarinol. Scandin.

2. Cyrenastrum Bourg. C. solida, costata, ligamento occulto. dentibus cardin. raluule dextra 1, sinistre 2 juxta positis.

† S. subsolidum Cl. (Öfrers. K. Vet. Ak. Förh., 1888). C. ovalis, angulis in toto orbitu nullis, modice rentrosa, subregulariter costata, sulcis concentricis pluribus priedita, umbones lati, rotundati, pars antica et positica concha subcquales. margo superior longus, curvatulus, inferior arcuatus. Long. $7^{1 / 2}$, alt. $5^{3 / 10}, \mathrm{cl}^{2}, 4^{4 / 5}-5 \mathrm{~mm}$.

Hab. Suecia, subfossitis in stratis postglacialibus ad Alnarp in prov. Skåne.

3. Corneola Cl. Concha tenuis, substriata et supius rugis incrementi nommllis. umbonibus ut Sphariastrum, liga. mento parvo $v$. fere occulto, dentibus cardin. vale. sinistre 2, pone positis, vale. dextre 1. omnibus in callo positis.

S. corneum Lin. (Srst. Nat. X, 1758, Tellina). C. subglobosa, subcordata, paullo infra medium ventrosa, sursum depressa, ad margines obtusa, corneo-cinerea, sæpe infra flara, striatula, antice paullo angustata, rotundata, postice latior, infra arcuata; umbones mediani, perlati, humiles; ligamentum obtectum; dens card. exterior valv. sinistra interiorem tantum ad dimidium tegit. Long. 10-14, alt. 8--11, cl. 7.-10 $\mathrm{mm}$.

Hab. Suecia vulgaris saltem usque ad $65^{0} 10^{\prime}$ lat. bor. Norv. mericlionalis. -- Dania et Fennia vulgaris.

Var. mucleus Stud. (Kurz. Verz., 1820): c. minor, globosa, inflata, ad margines rotundata, umbones latissimi, prominuli; long. 7, alt. 7 , cr. $6 \mathrm{~mm}$. - Suecia a pror. Skăne saltem in prov. Dalarne, rarius. - Dania tota (H. L.). -- Femia (e loco ignoto).

Forma orcula mh., c. globosa, inflata, ad margines obtusissima, infra umbones latissimos striatulos rufescentes fusca, striata et distanter sulcata, ad marginem inferum zona lutea lata; long. 13, alt. 10, cr. $9 \mathrm{~mm}$. - Norv. ad Kristiania.

Var. firmum Cl. ap. IV. (Famma Suec., 1873) : c. subglobosa, ventricosissima, sat firma, fuscocornea, sæpe fasciis Havis ornata. umbonibus latis prominentibus, dens card. exter. longissimus, interiorem omnino tegens; long. 11, alt. 81, ${ }_{2}$ (1). 
$71 / 2$ mm. - Dania in Jylland (prope Aarhuus a C. M. Ponlsen (letectum) nec non in Sjælland (Fingsted, Krogerup, Donse), Fyen (Korup) et ad Nykjöbing in Lolland a H. Lynge inventum).

S. ulicnyi II. (Naturf. Verein in Brünn, 1894). C. irregulariter ovato-cuadrangulata, paullo supra medium ventrosa, infra compressa, corneo-cinerea, fascia marginali lutea, forte striata, antice abbreviata, infra medium rotundata, postice longior, oblique truncata, pone ad basin acuminato-rotundata, margo dorsalis (inter umbones) brevis, rectus, ventralis subrectus; umbones tumidi, prominentes; ligamentum distinctum; dens card. exterior longissimus. Long. 12, alt. $10, \mathrm{cr} .7 \mathrm{~mm}$.

Hab. Suecia ad Stockholm (in lacu Trehörningen: V. L.). - Dania in Jylland (Horsens: H. L.). - S. scaldianum Auct. omnium, nec Gyclas scaldiana Norm.!

S. ovale Fér. (Ess. méth. Conch., 1807, Gyclas). C. orbiculatosubrhombea, subinæequilatera, angulata, vix ventrosula, ad margines acute compressa, eleganter striatula, nitida, flavocornea, tenuis, antice rotundata, post umbones dilatata, oblique descendens (subtruncata), marginibus (testre clausie) acutis, rectiusculis, inferiore solummodo arenato; umbones parvi, acutiusculi; dens card. exter. longissimus, dens card. valv. dextre subfurcata. Long. $7-8$, alt. $5^{1} / 2, c r .4 \mathrm{~mm}$. Forma major, I. 14, a. 7, $\mathrm{cr}^{*} .6 \mathrm{~mm}$.

Hab. Suecia sparsim a Skîne usque ad (Ovickjock in Lule Lappmark (67 ${ }^{\circ}$ lat. bor.). - Norv. circa Kristiania et ad Jedderen. - Dania in Jylland (Veile \&c.: H. L.). - Fenniu ad Kuopio (Jynkkï), in Lahafors, Borgnäs, ad Tammerfors, Carelia rossica (Tjudie): Hus. Helsingf.

S. mamillanum W. (Exposé crit., 1871). C. rotundato-ovata, parum ventricosa, maxime ad umbones tumida, deinde compressa, subtiliter striata, flavo-cornea, ad margines acute compressa, postice dilatata, truncata, orbitu rotundata, non angulata, margine infer. parum currato v. rectiusculo; umbones tumidi, obtusi, mitrellis magnis, planiusculis; dens 
card. exter. v. s. longissimus, interiorem fast omnino tegens. Long. 8, alt. $5^{1 / 2}, \mathrm{cr} .4^{1 / 2} \mathrm{~mm}$.

Hab. Srecia in Skåne (ad Vesum " prope Lund, etc.), Blekinge (ad Holmsjö), Westergötland (in rivulo Qvillebïcken pr. Göteborg), Dalarne (ad Kloster), Stockholm (in Långsjön: R. H.) Upland (ad Upsala), Jemtland (ad Offerdal: E. N.). - Norv. ad Vaale in Jarlsberg et in Fossanelv. - Dania in Sjælland (Fuursö, Gentofta, Gilleleje: H. Lynge, Kjöbenhavn) et Jylland (Veileaa: H. L.). - Fennia in Satakunta et Savonia boreali locis pluribus: in Kuopio, Tuusniemi, Nilsiä (Mus. Helsingf.).

Var. tomentosum W. 1. c.: C. straminea, dorso fusca, tomento tenui albido obtecta; long. 8, alt. 6, cr. 41/2 mm. - Suecia in Skåne (in stagno ad Wesum *), Westergötland (Borås: P. T. C.).

Var. suecicum W. (Fauna Suec., 1873): c. major, globosa, ventricosior, tenuis; long. 12, alt. 10, cr. $71 / 2 \mathrm{~mm}$. - Suecia in Skåne (Wesum *, Kristianstad), Blekinge (Romneby), Westergötland (Borås), Dalsland (Österby).

S. dupplicatum Cl. (Corr. blatt Ver. Regensburg, 18733). C. globosa, valde inflata, forte striata, flavidocornea, obtuse angulata, antice paullo angustior, breve rotundata, postice altior, oblicque subtruncata, margine inferiore regulariter curvato; umbones lati et alti, superne conchis minutis (mitrellis) forte prominentibus, convexis dupplicati; dens card. v. s. ext. interiore ad $3 / 4$ tegens. Long. 8, alt. $7^{1 / 2}$, cr. $6^{1} / 2 \mathrm{~mm}$.

Hab. Dania in Bornholm (ad llammershuus).

S. westerlundi Cl. ap. WV. (Fauna Suec., 1873). C. globosa, ventricosissima, maxime supra medium, ad margines acuta, tenue striata, sulcis pancis fuscis abbreviata, fulvocornea, nitida, infra fascia flavida, antice perbrevis, rotundata, postice brevis, marginibus omnibus curvatis, angulis obsoletis; umbones lati, superne sulco sicut mitrellatis, striatis; dens card. v. s. exterior interiorem ad dimidium tegens. Long. $71 / 2-8$, alt. $6^{1 / 2}-7, \mathrm{cr}^{2} .6 \frac{1}{2} \mathrm{~mm}$.

Hal. Suecia in Blekinge (Lângasjö ad Ronneby), in Dalarne (Dalbysjön * par. Säter, in Lillản ad Orsa), Upland (ad 
Upsala: J. E. Z., in Olandsân ad Stummelbo: P. de L., ad Ranüs: V. L.), Jemtland (ad Kall: E. N.). - Norv. in Osterdalen. - Fennia ad Kuopio (Jynkkä, Maljalampi: K. M. L.), Satakunta (Eura), ad Kittilä et in flum. Varsuga in Lapponia rossica (Nus. Helsingf.).

S. physale IV. (Nachrichtsbl. Mal. Ges., 1893). C. ovata, superne ventricoso-inflata, infra medium convexiuscula, ad margines compressa, obsolete striata, pallide citrina, antice regulariter rotundata, postice latior, rotundato-truncata, margine inferiore levissime curvato; umbones tumidi, lati, humiles, rotundati. Long. 8 , alt. $5^{1} / 2, \mathrm{cr} .4^{1} / 2 \mathrm{~mm}$.

Hab. Norvegia in Finmarken in fluvio Pasvig (et in flumine Tulom peninsulie Kola Rossiæ), invenit clariss. Ch. Rabot.

4. Calyculina (l. C. tenuissima, fragitis, striate $v$. striatulu, superne utinque angulata, margine superiore scepius recto, unbonibus infira latis, sursum anyustatis, valle prominentibus, superne mitrella duplicatis. dentibus card. vale. sin. 2, in valva dextra 1 , nullis in callo positis.

+ Umbones non inflati, nee incurvi, mitiella horizontali.

S. Iacustre Müll. (Verm. Hist. 11, 1774, Tellina). C. rhomboideoovalis, angulata, medio ventrosula, sursum lente inflexa, deorsum valde compressa, ad margines acutissima, alba v. flavescens, fragilis, nitida, antice infra paullo angustata, postice latior, subtruncata; umbones submediani, sursum breve celeriterque angustati, mitrella magna, inflata. Long. $7^{1 / 2}$, alt. $5^{1} / 2$, er. $3^{1 / 2} \mathrm{~mm}$. - Cyclas calyculata Drap.

Hab. Succia raro, ex. gr. ad Göteborg (Qvillebäcken, Mölndalsa et in »Stadsvassen»), Dalsland (in hortu pastoris ad Bäckebol: J. H.), ete. - Daniu sin lacubus et paludosis minus frequens $(0$. Müll.), Kjöbenhavn (in hortu botanico), ad Ruderhegn et ad Veile.

Var. mamillare Gass. (Moll. de liAgenais, 1849): c. minor, tenuissima, fragillima, albida, umbonibus leviter convexis, mitrellis maximis, forte inflatis. - Suecia in Östergötland, in Dalarne (ad Säter et in stagno Ruthens myra dicto). Fennia ad Furuby in Åland (Mus. Helsingf.).

S. pilacie W. (Fauna Binneneonch. pal. Reg. VII, 1890). C. ova- 
lis, non angulata, antice paullisper angustata, postice late rotundata, superne ad mitrellas ventricosa, dein convexa, ad margines compressa, acuta, tenuis, corneocinerea $\mathrm{r}$. infra flavescens, striata, nitens, margine supero leviter, infero sat forte arcuato; umbones convexi, humiles, mitrellis magnis v. maximis, horizontalibus $v$. extus dilatatis, forte striatis. Long. $4^{1} / 2$, alt. $3^{1} \frac{1}{2}, \mathrm{cr}^{2} 2^{1} / 2 \mathrm{~mm}$.

Hab. Suecia ad Ronneby (in stagno parvo pone riam ad Kallinge: et ad Stockholm (Gảngsätra: J. W.). Ceteroquin hiec species in Bohemia, Germania et Britannia, ubi tamen pro $\mathrm{S}$. ryckholti sumitur.

it Umbones non inflati. superne incurri, mitrellis intus oblique versis.

S. brochonianum Bourg. (Mon. Sphrer. Fr., 1854). C. oblongoovata, subrequilatera, angulata, tenuissima, superne ad umbones ventrosula, deinde celeriter et forte complanata, ad margines acutissima, corneocinerea, fascia et marginibus sxpe pallidis, antice deorsum angustata et producta, postice lata, oblique truncata; umbones sursum celeriter angustati, mitrellis parvis. Long. 10, alt. 8, or. $4 \mathrm{~mm}$.

Hab. Suecia ad Göteborg (in canali quadam in urbe, nec non in rivulo Qvillebäcken: C. calyculata var. A. Malm Göteb. Soc. Handl. III, 1855 cum fig.).

Var. steini A. Schm. (Zeitschr. f. Malak., 1858): c. rotundior, ventricosior, inferne compressa, superne minus distincte angulata, margine superiore curvatulo et mitrellis minimis. pars anterior conchæ multo minus producta; long. 7, alt. 6-7, cr. \& mm. - Forma major, 12-10-7 mm. - Suecia passim a Skăne usque in Dalarne (ad Säter). - Norv. meridionalis. - Dania in Jylland (Asmilds Have ad Viborg: C. MI. Poulsen, Horsens: H. Lynge); certe etian in insulis. - Fennia, ut ad ostium flum. Uleaielf, Frugård, Orimattila, Ingo et ad Finnby in ̊̊land (Mus. Helsingf).

S. ryckholti Norm. (Note nouv. Cycl., 1844, Cyclas) var. danicum Cl. ap. WT. (Fauna Suec., 1873). C. subtriangularis, incquilateralis, infra angulata, superne ventrosa, deinde lente et leviter compressa, ad margines acuta, cinereoflavida v. coerulescens, tenue striata, antice forte declivis 
et infra medium angustata, acuminata et producta, postice latissima, margine arcuato-descendens, ad basin rotundatoangulata, marg. superiore curvato; umbones lati, breves, sursum lente attenuati, mitrellis magnis, inflatis. Long. $7 \frac{1}{2}$, alt. 6 , cr. $4 \mathrm{~mm}$.

Hab. Suecia in Öland (ad Björnhofda). - Dania in Sjælland (Fuursö, Skjelskör \&c.) et Jylland (ad Viborg).

Forma major, long. $10 \mathrm{~mm}$. - Dania in Sjelland (ad Bröndsholmsdal et Boserup).

Var. strictum Norm. ap. Cl. Chemn. Conch. Cab., 1877): c. orbitu typi, ventricosissima, angulis rotundatis, umbonibus brevissimis, latissimis, mitrellis magnis; long. 5, alt $4^{1 / 9}$. cr. $3 \frac{2}{2} \mathrm{~mm}$. - Dania in ins. Bornholm.

\section{Gen. Pisidium C. Pfr.}

C. plus minusve incequitatera (antice semper longior), ligamento parti breviori insito. Animal siphone simplici, brevi, cylindraceo.

1. Fluminina $\mathrm{Cl}$. C. solida, costata, dentibus caidinatibus valvulre utrinsque duobus juxta positis.

P. amnicum Nï̈ll. (Verm. Hist. 11, 177', Tellina). C. valde inrquilatera, perobliqua, ovalis, sat ventrosa, nitida, fuscocinerea, zonis nigrescentibus, striato-sulcata, antice elongata, deorsum forte declivis et angustata, superne late rotundala, postice brevissima, subverticaliter truncata, convexiuscula, margo ventralis arcuatulus; umbones lati, parum prominentes. Long. 11, lat. 8, cr. $6 \mathrm{~mm}$.

Hab. Suecia, forma typica in Dalarne (in par. Gustaf et in Ljusterân ad Säter), in Upland (Upsala ad Flottsund) et ad Piteå. Nondum aliis locis peninsulre reperta. - Dania in Sjarland (locus orginalis Mülleri est amnis ad Fredriksdal). - Fennia in lacubus Ylüne, Saimen, Ladoga et Onega (Mus. Helsingf.).

Var. glaciale W. (Nachr. blatt Mal. Ges., 1893): c. brevior, latior, rotundior, margine ventrali forte arcuato, parte anteriore late rotundato; long. 9, alt. 7 , cr. $3^{3} / 4 \mathrm{~mm}$. - Fennia borealis circa $69^{\circ}$ lat. bor. (ad Ivalojokki ", Kamasjokki, Enare ice: Ch. R.). 
Var. elongatum Baud. (Ess. Mon. Pis. Fr., 1857): c. elongata, oblongo-ovata, valde costata, antice longius producta, umbonibus sat prominentibus, a margine posteriore magis remotis; long: 12. alt. $S_{1 / 2}^{1 /}, \mathrm{cr}^{1} \cdot 6^{1}, \mathrm{~mm}$. - Suecia in Skåne multis locis, Blekinge in maxime occidentali parte (in lacu Wesan), in Östergötland et Westergötland passim, in Upland (Upsala), Westmanland (in Mälaren et Svartån). - Dania in Sjælland multis locis (H. L.). - Fennia (in flum. Kunmo et Srir, ad Rautus et in Ladoga: Mus. Helsingf.).

Var. malni Cl. ap. W. (Fauna Suec, 1873): c. parra, subtilissime striata, sulcis nonnullis profundis fuscis, nitidissima, antice angustior, umbonibus acutiusculis, humilibus, magis a margine posteriore remotis, margine ventrali forte arcuato; long. $7 \frac{1}{2}$, lat. 6, cr. $4 \mathrm{~mm}$. - Suecia in Skåne (R⿳亠belöfsjön *, Rybelsdort' et in Ringsjön ad Ḱlinta) et Östergötland (ad Norrköping).

Var. striolatum Baud. (Ess. Mon. Pis. Fr., 1857): c. regulariter valde costata, umbonibus sat latis, a margine posteriore magis remotis, postice ad basin distinctissime angulata; long. 11. alt. S. er. $6 \mathrm{~mm}$. - Dania in Sjælland et Jylland late destributum (H. L.).

2. Rivulina Cl. C. solidula, striatula, dentibus cardinalibus valvila sinistre ¿, jurta positis, dente valv. dextro 1.

P. supinum A. Schm. (Zeitsch. f. Malak., 1850). C. valde inxequilatera, triangularis, solida, ventricosissima, tenue striata, sulcis profundioribus paucis, antice longe attenuata, forte angustata, margine superiore forte declivi, rectiusculo, apice rotundato, postice brevissima, fere verticaliter et recte truncata, margine inferiore curvatulo; umbones prominentes, paullo acuminati. Long. $4 \frac{1}{2}-5$, alt. 4 , cr. 亏े $\mathrm{mm}$.

Hab. Snecia in Upland ad Harg. - Dania in Sjælland (Sacsö: H. L.).

P. globulare Cl. ap. II'. (Fauna Suec., 1873). C. parum inaquilatera, rotundato-ovata, tenuis, ventricosa, subtiliter striata, antice brevis, rotundata, margine supero curvato, postice truncatulo-rotundata, margine inferiore sat arcuato; umbones bene prominentes, lati, rotundati. Long. 5, alt. $33^{1 / 2}$ 
-4, cr. $2^{1 / 2}-3 \mathrm{~mm}$. - Forma fere P. obtusali sed dentes alii.

Hab. Sueciu in Dahlsland (ad Hästefjord), in Dalarne (ad Säter, Hedemora ut etiam in regione alpina in Idre), ad Stockholm (Carlberg: J. W.), in Wermland (ad Arvika), Gestrikland (ad Gefle), Gotland. - Norv. meridionalis, saltem ad $62^{\circ}$ lat. bor. cum obtusali et fontinali frequentissima species sui generis. - Fennic in Kuopio (K. M. L.), ad Kinnula in Maaninka et ad Kaschkarantsa peninsulie Kola (Mus. Helsingf.).

3. Fossarina $\mathrm{Cl}$. C. tenuis, striata $v$. striatula, dentibus cardin. valvule sinistre 2, pone positis, dente valv. dextre 1 . t C. umbonibus superne plica compressa longitudinali proditis.

P. henslowianum Shepp. (Trans. Limn. Soc., 1823, Tellina). C. elongato-ovata, parum ventricosa, medio maxime, tenuis, dense regulariter striata, antice elongata, sat attenuata, rotundata, margine superiore lente longeque arcuatulo-declivi, postice brevis, rotundata, margine inferiore extense curvato; umbones prominentes paullo acuminati. Long. 5 . alt. 4, $\mathrm{cr}^{2}, 2^{1 / \mathrm{e}}-3 \mathrm{~mm}$.

Hab. Suecia sparsim et plerumque sat raro in Skåne, Östergötland, Westergötland, Nerike (ubi vulgatissima species sui generis), ad Stockholm, in Upland, Gotland et Westerbotten (in Sandön ad Piteå). - Norv. ad Bergen. ... Dania in Sjaclland pluribus locis (H. L.), Fyen (ad Nyborg).

Var. suecicum Cl. ap. W. (Fauna Suec., 1873): c. minor, oralis, parum producta et parum angustata, perlate rotundata, umbones prominentes, ad partem posteriorem magis approximati, plica umbonali valida; long. 4, alt, 3, cr. $2^{2{ }_{3}}$ mm. - Suecia ad Göteborg (in Qvillebäcken*). P. henslowianum Malm.

tt C. umbonibus non pliciferis.

*) C. rotundato-orata, umbonibus ad partem posteriorem approximatis, antice producta, rotundato-acuminata, postice abbreviata, rotundato-truncata.

a. - C. striatula.

P. parvulum Cl. ap. W. (Fauna Suec., 1873). C. orbiculatoovalis, ventricosissima, fragillima, antice rotundato-acuminata, postice rotundata, paullisper truncata, margine su- 
pero et infero valde arcuatis; umbones sat prominentes, acutiusculi. Long. 2, alt. $1 \frac{1}{2}$, cr. $1 \frac{1}{5} \mathrm{~mm}$.

Hab. Suecia in Blekinge (Ronneby in Långasjö ${ }^{*}$ ) et Helsingland (ad Saune),

Var. martensi CI. ap. W. 1. c.: c. major, solidior; long. $3^{4} / 5$, alt. $2^{4} / 5$, cr. $21 / 5 \mathrm{~mm}$. - Norv. ad Bergen.

b. - C. forte striata.

P. pulchellum Jen. (Trans. Phil. Soc. Cambr., 1832). G. oblique ovata, solida, sat ventrosa, dense et forte striata, nitida, antice paullo elongata et attenuata, margine supero forte declivi, apice rotundata, postice abbreviata, lata, subtruncata, inferne rotundato-angulata, margine ventrali arcuato; umbones subinflati, prominentes. Long. $3^{1 / 5}$, alt. $2^{3} / 5$, cr. $1^{4} / 5 \mathrm{~mm}$.

Hab. Suecia in prov. Skåne, Blekinge (loco unico ad Ronneby), Östergötland, Westergötland, Nerike, Stockholm, Upland (Ultuna et Temnaren), Westmanland (Persbo pr. Westerås), Gestrikland (ad Gefle), Herjedalen (Ytterhagdal), Dalarne (par. Gustaf et Husby), IVesterbotten (ad Piteå), Gotland (ad Visby). -Norv. meridionalis et supra $62^{\circ}$ lat. bor. multis locis. - Danic in Sjæelland (ad Priestö) et Jylland (in Nörreaa). - Femia in Satakunta in par. Virmo (Mus. Helsingf.).

P. nitidum Jen. (Trans. Phil. Soc. Cambr., 1832). C. orbiculatoovata, paullo obliqua, solidula, compressiuscula, eleganter dense striata, sulculis nonnullis sub umbones, nitidissima, antice attenuata, late rotundata, margine supero extense declivi, postice rofundata, margine inferiore sat arcuato; umbones depressiusculi, lati, rotundati. Long. $2^{4} / 5$, alt. $2^{1 / 5}$ cr. $1^{4} / 5 \mathrm{~mm}$.

Hab. In Suecia hæc species inter rariores enumeranda est, occurit in Skâne (ut in Rönneå), Westergötland (in (Qvillebäcken pr. Göteborg), Upland (Teljöbäck par. Áker), Dalarne (Lillån par. Orsa), Jemtland (ad Krokom) et hine inde in provinciis interjacentibus. - Norv. meridionalis et media multis locis, etiam supra $62^{\circ}$ lat. bor., vulgaris, in lacubus montium alte sitis usque in Westfinmarken (in ins. Andö, $69^{\circ} 33^{\prime}$ l. b.). - 
Dania tota (H. Lynge). - Fennia in flum. Yläne, ad Helsingfors, Luhanko, Sääminki, Rantasalmi, Kuopio (Mus. Helsingf.).

Var. fedderseni W. (Fauna Binnenconch., VII, 1890): c. tantum sub umbones convexinscula, de cœetero forte compressa, marginibus acutis, tenue et anguste striata, umbones magni, planoconvexi, paullo laterales, infra abscissi; long. 3, alt. $2^{3 / 4}, \mathrm{er} .1^{1 / 4} \mathrm{~mm}$, - Islandia.

P. lilljeborgi C.l. (Malak. Blätt., 1886). C. oblirue ovalis, triangularis, inflato-ventricosa, irregulariter striata. sulcis et incisuris pluribus, antice acuminato-rotundata, margine brevi declivi, postice brevissima, truncato-potundata, margine dorsali convexo, ventrali forte arcuato; umbones inflati, late rotundati, valde prominentes, sæpe superne late mamillati. Long. 4, alt. 3 , cr. $2^{1} / 2 \mathrm{~mm}$.

Hab. Suecia in Jemtland (Frösön: E. N.). - Norregia priecipue borealis multis locis inter Circulum polarem et Hare glaciale. - Istundia. - Fennia in Lapp. ross. ad Olenitsa (Mus. Helsingf.).

Var. transcersale Cl. 1. c.: c. obliruior, antice magis attenuata et acuminata, postice fortius truncata, margine rentrali longiore, antice altius ascendente, umbonilus tumido-inflatis. latioribus et magis prominentibus. - Norv. Troldwand in Trondenæs.

Tar. hoyeri Cl. l. c.: c. orata, forte striata, antice abbreriata, obtusa, postice breve rotundata, superne angulata. - Norr. in ins. Tromsö et Renö.

†P. lindströmi Cil. (Öfver's. K. Sv. Vet. Ak. Förh., 1888). G. oblique ovata, valde inrquilatera, ventricosa, sat regulariter striatula, sulcis pluribus $(4-6)$ profundioribus predita, antice producta, paullo angustata, late rotundata, postice forte rotundata, margo dorsalis convexus, ventralis forte arcuatus, umbones rotundati, prominentes. Long. 4, alt. 3, cr. $2^{1} / 2^{2}-2^{4 / 5} \mathrm{~mm}$.

Hab. Succia, subfossitis in stratis postglacialibus ad Alnarp et Hofgârd in prov. Skảne.

**) C. oblongo-orata. striatula $\because$ striata, antice paullo angrstata, rotundata, postice brevis, late rotundata, umbonibus ad partem posteriorem apmoximatis.

P. fontinale C. Pfr. (Naturgesch., 1821). C. ovata, inflato-ventricosa, tenuis, nitida, striatula, antice paullo elongata, ro- 
154 C. 4. Westerlund, Synopsis Molluscorum Extramarinor. Scandin.

tundata, margine supero arcuato, postice rotundata, ad basin obsolete angulata, margine rentrali curvato, umhones lati, prominentes. Long. 4, alt. $2^{4} / 5$, cr. $3 \mathrm{~mm}$.

Hab. Suecia meridionalis et media, saltem usque in Jemtland (Frösön: E. N.). - Norv. merid. \& media vulgaris. Dania passim. - Eennia in par. Sysmä et Salmi, ad Nikola ad fl. Srir, ad Suma in Car. ross. (Mus. Helsingf.).

Var. acuminatum Cl. ap. W. (Fauna Suec., 1873): c. parum rentricosa, antice magis acuminata, postice truncatula, umbonibus prominulis; long. $3^{1} \frac{1}{2}$, alt. 3, cr. $2 \mathrm{~mm}$. - Suecia in Skåne (Lefrasjön) et Dalarne (Säter*).

Var. flavescens Cl. (Mon. in Chemn. Conch. Cab., 1879): c. ventricosissima, parte anteriore angustiore, umbonibus ralde prominentibus; long. $4^{1 / 2}$, alt. $3^{4} / 5$ : cr. $3^{1 / 5} \mathrm{~mm}$. - Suecia in Upland (ad fabricam Forsmark: P. de L.).

P. cinereum Alder (Cat. suppl., 1837). C. elongato-ovalis, fere medio alta, subrequilateralis, antice paullo longior et angustior, rotundata, postice latius rotundata, ad vel infra medium ventricosa, inferne, valvis clausis, compressa, acuta, cinerea, ad marginem inferiorem albescens, tenuissime striatula, marginibus omnibus arcuatis, angulis nullis; um. bones prominentes, rotundati. Long. 4-6, alt. 3-4, cr. $2-3 \mathrm{~mm}$.

Hab. Dania in Sjalland (ad Bröndsholms-dal et specim. plur. e locis ignotis: O. Mörch).

P. pallidum Gass. (Descr. Pisid. Aquit., 1855). C. perobliqua, ovata, subcuneiformis, supra medium valde ventricosa, sursnm breve, deorsum longe declivis, flavidocornea, flavolimbata, striata, nitida, antice late rotundato-attenuata, mar. gine super. forte declivi, arcuatulo, postice brevis, rotundata, margine ventrali arcuato; umbones valde prominentes, attenuato-rotundati; ligamentum perdistinctum. Long. 4, alt. $3^{1 / 3},(r) .2^{1 / 2} \mathrm{~mm}$. (5-4-3 mm).

Hab. Suecia prope Lund. - Norv. in Bondtjern in Asker.

- Dania in Bornholm.

P. Iovéni Cl. (Moll. Faun. Oester.-Ung., 1890). C. oblique rotundato-ovata, rentricosissima, non angulata, flavescens, zo- 
nis multis fuscis, striatula, antice angustata, rotundata, margine superiore bene arcuato-declivi, postice brevis, late rotundata, margine rentrali ralde arcuato; umbones sat inflati. Long. $41 / 2$, alt. $4, \mathrm{Cl}^{2} .3 \mathrm{~mm}$.

Hab. Norregia in parte aretica frequens. De cactero solummodo in Helvetia, 2400 m. s. m.

P. arcticum II. (Nachr. blatt Mal. Ges., 189:3). C. oblicfue oralis, antice rotundato-angustata, postice subperpendiculare truncata, duplo brevior, superne vix ventrosula, infra medium compressa, fusco-cornea, dense costulato-striata, subsulcata, locis 3-i profundius incisa, margine dorsali et ventrali subaequaliter arcuatulis; umbones humiles, apicibus minutis connirentibus. Long. 6, alt. $4^{1 / 2}, \mathrm{cr} .2^{1 / 2} \mathrm{~mm}$. Hab. Norregia in Sydvaranger ad Bosijare prope flumen Pasvig, $69^{\circ}-70^{\circ}$ 1. b. (Ch. R.).

***) C. ollique acuminato-ovata $i$. subcuneiformis, valde incequilatera, postice brevissima, verticaliter truncata, $u m-$ bonibus valde posticis.

P. subtruncatum Malm (Göteb. Tet. Saml. Handl. 1853). C. obli. que ovalis, inflate rentricosa, maxime infra medium, deinde lineis subrectis in angulum rectum declivis, tenuissime striatula, apicibus lavis, antice plus quam duplo longior, lata, margine sensim arcuato-declivi, rotundata, margine postico leviter arcuato, dorsali et rentrali sat arcuatis; umbones late rotundati, parum prominentes. Long. $3^{1 / 4}$, alt. $2^{3} / 5, \mathrm{cr}^{2} .2 \mathrm{~mm}$. - P. subtr. r. turgidum Cl. in Mest. Fauna Suec.

Hab. Sueciu in prov. Skinne, Blekinge (Ronneby), Westergötland (Göteborg ${ }^{*}$, Östergötland, Stockholm (Djurgardsbrunn ad Blå Porten et in lacu Mälaren ad 36-39 met. prof.), Upland (Lpsala et Harg), Dalarne (in lacu Husbysjön prope Sïter), Westerbotten (ad Röknäs pr. Piteå). - Norv. ad Kristiania, Kristiansand (ad »Odderö»), Eger in Modum et ad Bergen. Diniu in Sjaelland (Donse, Sorö, Ravenzes) et Jylland (ad Horsens: H. 1..), ad Ny̧borg (fide Mörch). - Fennia in rivulo Pappilanoja in Kuopio (Mus. Helsingf.). 
Var. sphenoides West. (Fauna palärarkt. VII 1890): c. peroblique trigono-ovata $\nabla$. cuneiformis, modice ventricosa. distinctius striata, etiam in apicibus, antice rostriforme peranguste acuminata, apice pone basin, postice brevissima, alta, supra et infra angulata, verticale subrecte truncata, margine ventrali subrecto, umbones acutiores, altiores; long. $3-31 / 2$, a. $21 / 3-21 / 2$, cr. $13 / 5 \mathrm{~mm}$. - Destributio eadem ac typi. (P. subtruncatum Cless. in West. Fanna Suec. 1873).

Var. poulseni Cless. (Nal. Blätt. 1878): c. tenue irregulariter striata, corneoflavida, antice acuminata, ad aream et areolam vix angulata, umbones ventricosi, panllo prominentes; long. 3, a. 21/2, cr. $2 \mathrm{~mm}$. - Dania ad Fjerdetsen et Bygholm prope Horsens.

P. scholtzi Cless. (Malak. Blïtt. 187L). C. abbreviato-orata, postice brevissima, in linea subrecta et verticali truncata, ad basin rotundato-angulata, ventricosissima, pone ad marginem ventralem abrupte compressa, valvis angulum obtusissimum formantibus, antice late attenuato-rotundata, margine supero lente arcuatulo-descendente, ventrali strictiusculo, tenue et regulariter striata; umbones lati, valde prominentes, sæpius ad apicem late ef forte mamillati. Long. 3, alt. $2^{3} / 5$, cr. $2^{2} / 5$ mm.

Hab. Suecia ad Ronneby (in Lângasjö), ITpsala (P. T. C..). in Dalarne (Breisväg par. Orsa: P. de L., Styggforsen: P. T. C), Westerbotten (Strömnäs ad Piteå). - Norv. in Gudbrandsdal (Bakkejordet in Ringebu et "Högdatjern» in Tönset ad 840 in. alt.

Var. lapponicum Cl. ap. IV. (Fauna Snec., 1873): c. minima, globosa (æque crassa ac longa), striata et rugis increrrenti pluribus, margine inferiore areuato, umbonibus magnis, tumidis. - Suecia in Dalarne (ad Långhyttan et Kebble prope Hedemora), Jemtland (ad Östersund: E. N.) et Lappland (unde Cless. formam a cl. Keitel lectam descripsit).

:***) C. subquadriangulata, whomboidea, parte anteriore tantum paullo longiore, margine ventrali subrecto, postico aiveruto.

P. milium Held (Isis 1836). C. sapius ventricosissima, ad basin angulum obtusum formans, tenuissime striata, nitidissima, margine dorsali medio subrecto, anteriore regulariter extense arcuato et ventrali subrecto ad basin protracta et 
rotundate angulata, postice supra et infra angulata; umbones lati, tumidi, convexiusculi. Long. 3, alt. $2^{1 / 3}, \mathrm{cr}$. $1^{5 / 6} \mathrm{~mm}$.

Hað. Sueciu in Skảne (Lund, Helgeå, Rönneå), Blekinge (Ronneby plur. locis, Augerum etc.), Westergötland (in (2villebäcken ad (7öteborg et Boräs), Bohuslïn (in rivulo ad molinam Skaftö), Östergötland (Omberg, Mjölsefall), Dalsland (Billingfors), ad Stockholm (Gångsïtra: V. L.), Upland (Harg), Westerbotten (in Sandön pr. Piteá). - Norvegia multis locis, ut Bergen, Skien (in Limkjern), Arendal, Kristianssand, Kristiania, Norderhoug, Ringebu, »Kjemsö» ad Koppang, Gravdal in Valders etc. Dania in Sjalland (Jonstrup Hegn, Sörup, Kjöbenhavu) et in Jylland (Horsens): H. L., nec non in Fyen et Bornholm. Fennia in palude Maljalampi pr. Kuopio et ad Vosnesenje in Onega (Mus. Helsingfors).

Tar. oidaleum C. G. West. (Ronneby Flora och Fauna 1890: c. valde ventricosa, maxime paullisper sub medio, deinde valvis subplanis angulum maximum (c. $130^{\circ}$ ) ad marginem formantibus, forte striata et nitidissima, antice abbreviata. parte posteriore tantum paulo longior; umbones lati, rotundati, superne sæpius prærupti. -- Suecia ad Ronneby (in lacu Långasjö).

Var. unioides West. (Fauna Suec. 1873): c. orbitu Unionis crassi. parte anteriore multo longiore et acutiore, margine poste riore forte arcuato, sed margine ventrali fere recto, umbones prominentes. - Suecia in provinciis quam maxime borealibus, Lappland, Jämtland et Norrbotten. - Norregia ad Bergen et Arendal. - Fennia in Car. ross. ad Tjudi (Mus. Helsingfors).

****) (. suborbiculuto-ovalis, umbonibus magis medianis, parte anteriore tantum paullo longiore et angustiore, margine superiore et anteriore bene, posteriore et inferiore forte arcuatis.

P. obtusale (Lam?) C. Pfr. (Naturg. D. Moll. 1. 1821). C. suborbiculata, tenuis, tenuissime striata, nitidula, tantum antice superne minute angulata, sat regulariter forte ventricosa, fere infra medium maxime, valvis ad marginem ventralem angulum obtusum $\left(90^{\circ}-125^{\circ}\right)$ formantibus; umbones ralidi, convexi, lati, prominentes. Long. $3^{1} \frac{1}{2}$, alt. $2^{1} / 2, \mathrm{cr}^{\mathrm{r}}$. $2^{3} / 10 \mathrm{~mm}$. 
Hab. Suecia per totum regnum usque in Lapponiam quam maxime septentrionalem. - Norv. saltem usque in Nordlanden vulgaris. - Dania tota vulgaris. - Femnia meridionalis et media.

Var. magnificum Cless. ap. West. (Famna Suec. 1873): c. major, magis ovata, inflata, parte anteriore magis elougata, umbonibus maximis, rotundatis, valde prominentibus; long. 5. alt. 4, cr. $3 \frac{1}{2} \mathrm{~mm}$. - Suecia ad Göteborg*, ad Stockholm (Haga: Hj. W.) et in Upland (ad Harg). - Norr. ad Bergen.

Var. fragile Cless. ap. West. 1. c.: c. parra, tenerrima, ventricosissima, nitida, striata; long. $3 \frac{1}{5}$, alt. $3, \mathrm{cr} .2^{3} / 10 \mathrm{~mm}$. - Suecia ad Ronneby.

Var. solidum Cless. ap. West. 1. c.: c. solida, flavida, tenuiter striata, splendida; long. $3^{3 / 5}$, alt. 3 , cr. $2^{1 / 2} \mathrm{~mm}$. - Norvegia ad Bergen. - Dania in Sjelland (Fuirendal, Holte, Ellemose ad Valdbygaard, Silketorp).

Var. esmarkianum Cless. (Malak. Blätt. 1883): c. magna, tenuissima, forte irregulariterque striata, diasomatibus pluribus magnis, parte anteriore angustato, rotundato, posteriore abbreviato, rotundato, umbonibus latis, inflatis. - Norv. al Iristiania, Herstad in Hof et Jarlsberg.

P. personatum Malm (Göteb. Samh. Handl. 1853). C. tenuissime striata, sulcis profundioribus $1-5$ præcdita, nitidissima, compressiuscula, supra medium maxime ventricosula (valvis leviter et sat regulariter convexis, deorsum parum compressis, angulum obtuse-acutum formantibus); umbones subprominuli. Long. $3^{2} / 3$, alt. $3^{1 / 5}, \mathrm{cr}^{2} .2^{1} / 3 \mathrm{~mm}$. Hab. Suecia ad Göteborg (in rivulo ad Hofås), Ronneby etc. - Norvegia ad Romerige. - Dania in Sjælland (Borup: H. L.), Jylland (Visborg, Nörreaa). Islandia. - Fennia in Solovetsk (Hus. Helsingfors).

P. pusillum Gmel. (Syst. nat., XIll 1788, Tellina). C. orbiculatoovalis, striatula, infra striis paucis profundioribus, vix nitidula, sat solida, antice late rotundata, compressiuscula, valvis regulariter convexiusculis, ad marginem ventralem angulum subacutum formantibus; umbones lati, rotundati, prominuli. Long. $3^{1} / 2$, alt. 3 , cr. $2^{1} / 4 \mathrm{~mm}$.

Hab. Suecia ad Ciöteborg multis locis, Bohuslän (in pa- 
lude ad molinam Skaftö: P. de L.), ad Stockholm (Gảngsätra: V. L.), Westerbotten (ad Piteå). - Norv. ad Kristiania ad Hougsund in Modum. - Damia in Sjælland (Bröndholmsdal), Fyen (fide H. L.), Jylland (Viborg et Nörreaa). - Isluntia. Fennia ad Haapaniemi pr. Kuopio, Telekina in Carelia ross., Tetrina et Varsuga in Lapp. ross. (Mus. Helsingfors).

Var. feröense M[örch (Syn. moll. Dan. 1864): c. lenticularis, suborbicularis, antice prolongata, subangustata, postice arcuata, obsolete biangulata, hyalina, intus limbo lacteo; unibones ( $t$. fotalis) ovales, nitidissimi, parum prominentes, sape utrinque radio abrupto limo ferrugineo; long. $4^{1 / 4}$, alt. $4 \mathrm{~mm}$. Forma qualis in P. pusillo, umbones ut in P. nitid̄o. (Mörch). - Ins. Færö.

\section{Fam. Unionidx.}

I. pallio antice et inferne aperto, postice comato, uperturis duabus non ut siphones elongatis. Concha magna, oblonga, incequilatera.

Obs. Formas tantum nonnullas et quam maxime principes hueusque cognitas hujus valde variabilis familiæ explicare volo, sint pro lubitu uno species, altero rarietates, comprehensiones in his magis quam alibi vaguæ. Nulla pars nostræ faune tam incognita, tam inexplorata est et tamen abundant regna borealia formis generum Unio et Anodonta.

\section{Gen. Unio Retz.}

C. crassa, ponderosa, dente cardinali valve destrce simplici, valce sinistre bijartito, dente laterali sub ligamento lamelliformi longo, solitario in valva dextra, duplici in valva sinistra.

+ Umbones undulato-rugosi.

*) C. postice non dilatata. fusca, varo et obsulete radiata. crassa et ponderosa, umbones rarissime integri, supius profunde decorticati. dens card. conicus, trigonus crassus, crenatus, vel elongatus, compressus. sed crissus.

U. crassus Ritz. (Nov. test, gen. 1788). C. ovalis v. elongatoovata, olivaceovirescens, antice late rotundata, producta, postice circa duplo longior, angustata, margine posteriore arcuato-descendente cum $m$. ventrali fere toto recto aut 
medio subretuso rostrum rotundatum v. subtruncatum fere basale formante; dens card. verticalis, conicus, trigonus.

Typus: C. oralis (non orata), ventricosa, crassa, fusca v. virescens, raro obsolete radiata, margine dorsali magis enrvato, umbonibus depressis, natibus decorticatis, læribus; long. 50-60, alt. $30-37$, ext. $20 \sim 26 \mathrm{~mm}$. - Rossm. Icon. f. 126,411 .

Hab. Suecia in prov. Skåne et Östergötland. - Dania in Sjælland et Jylland. - Femia meridionalis.

Var. acutus Rossm. (Icon. f. 413, 1837): c. elongata, acute orata, ventricosa, fuscocastanea, nitida, solida, postice in rostrum basale obtuse acutum, declinatum producta, margo ventralis fere rectus, posterior forte arcuato-descendens, umbones turgidi, area elliptica, planiuscula, dens card. subcompressus, lamella crassæ; long. 80-87, alt. $47, \mathrm{cr} .36$ mm. - Suecia in Skåne (Käflinge in amne et in palude ad Dagstorp).

Var. mbens Mke. (Syn. 1830): c. brevis, subtrigono-ovata, superne subventricosa, inferne complanata, fusco-nigra, antice late rotundata, postice celeriter in rostrum basale acuminata, margo dorsalis arcuatus, ventralis rectus, umbones prominuli, decorticati, dens card. subcompressus, lamella arcuatie, exsertæ; long. 60, alt. 40, cr. $25 \mathrm{~mm}$. - Snecia in Skine (in amne ad Käflinge). - Rossm. Icon. f. 412.

U. barys mh. C. elongato-ovata, ventricosa, crassa, ponderosa, fusco-olivacea, viride radiata, antice producta, angulata, rotundata, superne regulariter extense convexa, postice $2^{1 / 2}$ longior, lente utrinque in rostrum inframedianum acuminato-rotundatum producta, margo ventralis medio rectus; umbones forte undulati v. decorticati; dentes card. ut L. crassi, sed juxta positi et multo validiores, fossula interposita valv. sin. lata, non profunda. Long. 70, alt. 45-50, cr. 38 (100-54-42: Kob., 90--50-30: Cless.) Cifr. West. Fauna paläarkt. VII, 1890 , p. 63. - U. pseudolitoralis Cless. 1875, nomen hybridum!

Hab. Dania in maxime meridionali parte peninsulæ Jỵlland (locus originalis est Tapsaaen "ad Kristiansfeld in maxime septentrionali parte Schlesvigice).

U. ater Nilss. (Hist. moll. Su. 1822). C. ovato-oblonga, antice 
ventricosa et crassa (priesertim parte inferiore antica), postice compressa, sub epidermide atro nitida, argentea, margine dorsali ventralique subparallelis et xqualiter arcuatulis (vel illo longe arcuatulo, hoc substricto v. medio retusulo), parte anteriore brevissimo et posteriore triplo longiore fere reque latis, rotundato-truncatis; umbones depressi, decorticati; dens card. dilatato-conicus, crassus, peroblicquus, intus convexus, extus planus et striatus. Long. 80 (ant. 20, post. 60), alt. 40, cr. $26 \mathrm{~mm}$.

Hab. Suecia in Skåne (in Höjeå et Köpingeå), Östergötland (in Svartån ad Boxholm, Storân ad Söderköping, Skenån ad Skeninge et forma minor ad Solberga). - - Fennia in par. Tenala (Mus. Helsingf.).

Var: subdecuvatus West. (Exposé crit. 1871): c. oblongo-orata, ventricosa, viridifusca, postice compressa, elongata, subdecurvata, rotuudato-truncata, margo ventralis plus minus retusus, umbones tumidi, dens card. compressus, crassiusculus, crenatus; long. 50-60 mm. Forma inter U. decurvatum Rossm. Icon. f. 131 et U. atrovir. Rossm. f. 206 media. - Suecia in Skåne (Käflingeå *) et Östergötland (Motala).

Var. dannemoree (Mörch) Bourg. ap. Loc. (Catal. géner. 1882): c. angusta, valde elongata, supra et infra parallela, ubique fere requaliter ventricosa, atra, crassa, antice angusta, rotundata, postice quadruplo longior, in rostrum medianum sat acutum producta, parte anteriore brevissimo, umbones parum convexi, dens card. crassissimus, trigonus, ad marginem superiorem forte approximatus, semper sine denticulo præposito, margarita flavidoplumbea; long. 70, alt. 30, cr. 25-27 $\mathrm{mm}$. - Suecia in Upland (ad Dannemora).

Var. septemtrionalis Bonrg. ap. Loc. 1. c.: c. elongatulo-ovoidea, atrovirescens, parum ventrosa, parte anteriore angustato, rotundato, posteriore $23 / 4$ longiore, paullo dilatato, in rostrum subbasale perobtusum producta, margine dorsali elongato-arcuatulo, posteriore declivi, basali rectiuseulo, umbones lati, obsoleti, detriti $v$. decorticati, dens card. sat fortis, trigonus; long. 52-55, alt. 27-31, er. 17-.21 mm. - "Cette forme appartient à un groupe de Nayades snédoises dont plusieurs se retrouvent en France" (Bourg.).

U. elegans IVest. (Nalak. Blätt. 1867). C. longa, ad tres ante- 
162 C. A. Westerlund, Synopsis Molluscorum Extramarinor. Scandin.

riores partes altitudine subrequalis, marginibus parallelis, fuscopicea, rufescens, forte reneo splendida, numquam radiata, ad umbones ventricosa, infra et præcipue rostro longo mediano, utrinque angustato apice subtruncato forte compressa; pars antica perbrevis, humilis, postica plus quam quadruplo longior, margine ventrali postice ascendente; umbones lati, humiles, decorticati; ligamentum validum; dens card. crassus, elongatus, fere longitudinalis, crenatus, lamellæ crassie. Long. 110 (ant. 20, post. 85), alt. 42 , cr. $30 \mathrm{~mm}$.

$\mathrm{Hab}$. Suecia in Östergötland (in lacubus Föllingarne*, Kisasjön et Kilarpsjön). Forma æegre interpretenda, ad tumidum vergens.

**) C. postice dilatata ct superne plus minus angulata, fuscovirescens, supe pulchre saturatius viridc radiate et fuscozonata, crassiuscula, umbones sapissime integri, dens cord. parvus, compressus, obliquus.

U. batavus Mat. \& Rack. (Trans Soc. Linn. 1807). C. oblongoovalis, postice dilatata, sat ventrosa, inferne et postice compressa, antice rotundata, postice $22^{1} / 2$ longior, in 10 strum latum, linguxforme, fere medianum, subtruncatum producta, margine dorsali areuatulo, ventrali subrecto; umbones prominuli, ventrosi; ligamentum angustum, longum. Long. 60-70, alt. 30-35, cr. $20-25 \mathrm{~mm}$. - Rossm. Icon. f. 128 a.

Hab. Suecia in Skåne (Höjeå, Köpingeå, Almaå \&c.), Östergötland (in Stångån) et Dalarne (in lacı Hyen, par. Schildvie). - Dania in Sjaclland (Ringstedaa \&e.) et Jylland.

Forma minor (long. 40-50, alt. 25̃-28 mm.), obscurior, subolivacea, zonis multis atrobrunneis, postice latior, subrhombea, margine dorsali magis curvato, basali subretuso, in Höjeå et in Skenån occurrit.

th Umbones tuberculato-rugosi, postice margine areali inferiori (callo obtuso) obliquo utrinque ab area distincti. Denscardinalis valva dextre compressus, sat crassus, longitulinalis. posteriore value sinistre valido.

U. tumidus Retz. (Nov. test. gen. 1788). C. ovato-conica, ven- 
tricosa (rostice compressa), crassa, ponderosa, brunnea vel rufofusca, siepius fuscoradiata et nigrozonata, antice lata, rotundata, postice $2^{1 / 2}$ longior, in rostrum acute conicum, medianum, utrincue forte stricteque acuminata, margine dorsali rectiusculo, antice et postice sæpius angulato, ventrali plus minus arcuato; umbones prominentes. Long. 75 , alt. 3ัว, $\mathrm{Cl}^{2} .25 \mathrm{~mm}$.

Hab. Srecia in plerisque provinciis merid. et mediis usque in Dalarne. - Mumiu in plerisque Iacubus et amnibus. Fennia merid. et media.

Forma major (Rossm. Icon. f. 402): long. 110, alt. 50, cr. 35. - Rarior.

Var. mïlleri Rossm. (Icon. t’. óf1, 1838): e. acute ovata, forte compressa, lutescens, nigricante cingulata, antice prolongata, late rotundata, postice in rostrum acutum medianum elongata, margine dorsali et ventrali subreque arcuatulis, umbones tumiduli, medio approximati, dentes card. compressi, elongati, humiles; long. 67-70, alt. 38-69, cr. 18 -20 mm. - Dania.

Forma compressa Mörch (Syn. moll. Dan. 1864): c. minus compressa, margine posteriore in rostrum breve conicum fere basale forte declivo, margine ventrali subrecto; long. 56-60, alt. 33 , cr. 22 mm. - Dania in Sjalland (Tjustrupsö et Barelsesö). - Rossm. Icon. f. 739 .

Tar. oralis Mont. (Test. Brit. 1803 , Mya): c. oblique trigonoorata, antice brevis, humilis, rotundata, post umbones margine lente regulariter arcuato-declivi et ad basin cum margine ventrali antice arcuato-ascendente, postea recto rostrum obtuse acuminatum formante, superne ad umbones rentrosula. de cretero forte complanata, lumula superne ante umbones) magna, profunda, ovalis, dentes card, et lamellæe angulum validum profundum obtusum formantes; ex. max. long. 65 (ant. 12, post. 5̌3), alt. 35, cr. $22 \mathrm{~mm}$. Suecia in Skine.

Tar. lacusti. Rossm. (Icon. f. 542, 775): c. postice magis elongata et angustata, infra post medium compressa, rostro an«uste attenuato, inframediano apice truncato, subdecurrato, suleis incrementi rostri hiantibus, margine ventrali post medium rectiusculo, ante apjeem retusulo; long. 70, alt. 3:3, cr. 2t mm. - Suecia procipue in pror. Skåne.-- Dania in Endrupsö. 
Var. limicola Mörch (Syn. moll. Dan. 1864): c. ovali-piriformis, longa, castaneo-nigricans, mnicolor, margine dorsali et rentrali subparallelis, parte posteriore medio obtuse conico, dentes card. crassi, dens card. valva dextra subtransrersalis, sed dentes sæpe morbosi et umbones sipe decorticati; long. 85, alt. 40, cr. $26 \mathrm{~mm}$. - Suecia in Skåne, Smàland et Östersötland (in lacubus fundo limoso). Forma minor et elonyata ad Stockholm (in loco Tyska botten dicto: V. L.). - Dania in Sjælland. - Fennia merid. et media. - Rossm. Icon. f. 20t, Nord. \& Nyl. Finl. Moll. f. 73 .

Var. conus Spengl. (Skrivt. Nat. hist. Selsk. 1793): c. oblique cuneiformis vel orato-piriformis, ventricosa, viridibrunnea, antice rotundata, sat prolongata, postice $2 \frac{1}{2}$ longior, in rostrum inframedianum vel subbasale angustata, margine posteriore regulariter extenso ad basin descendente, rentrali recto, antice ascendente, dens card. subrectangularis, crassus; long. 52-64, alt. 27-32, cr. $20-25 \mathrm{~mm}$. - Suecia in Skåne. - Dania in Sjælland in plerisque lacubus majoribus fundo arenoso). - Fennia, Kotojärvi in Borgnäs (Mus. Helsingfors).

Var. pictus Beck, Mörch (Syn. moll. Dan. 1864): c. oblique cuneiformis, olivaceo-viridis, ventricosa, parte anteriore brevi subrotundato, posteriore triplo longiore in rostrum inframedianum sat acutum elongato, margine superiore ad basin bene arcuato, ventrali elongato-arcuato, umbones ralide tuberculati, tumidi, dens card. brevis, subtrigonus, crassus; long. 4S-50, alt. 24-28, cr. 16-18 mm. -- Suecia in Skåne (ut in Käflingeå). -.. Dania in Ladegaardsaaen ad Kjöbenhavn.

Var. tumidulus Loc. (Contrib. XIII, 1889): c. ovalis, sat brevis, flaroviridis, obscurius radiata, mediocriter ventricosa, antice rotundato-descendens, superne angulata, postice duplo longior, in rostrum medianum, breve, sat acutum producta, margine superiore parum arcuato, descendente, ventrali elongato-curvato, antice fortius, umbones sat prominentes, dens card. subconicus, altus, tenuis, sat longus; long. 62 -68, alt. 33-35, cr. 23-25 mm. - Suecia in Skine Fennia ad Rantasalmi (Mus. Helsingf.). - Mya ovata Denovan, U. tumidus Rossm. Icon. f. 203, Dup. Hist. moll, t. 38 f. 20.

Var. saccatus Rossm. (Icon. f. 969, 1859): c. oblonga (part. posteriore ad quintuplo longiore), antice prolongata, angustata, rotundata, deinde usque post medium dilatata, po- 
stice in rostrum medianum obtusum angustata, margine superiore toto regulariter extense convexo, ventrali usque ad medium stricto, deinde saccato, postea oblique stricteque ad medium ascendente; long. 75, alt. 36, cr. 25 mm. - Suecia in Skåne (Köpingeå), Småland (in Wersjön, Bodasjön, Emån et Hulingen), Östergötland (in Kisasjön, Föllingarne, Åsunden, Kö̈fleforsån, Hargsjön, Svartån etc.).

Hit Umbones tuberculati. Dens card. valure dextrce compressus, tenuis, lamelliformis, longitudinalis, posteriore valve sin. minimo vel nullo.

U. I'ostratus Lam. (Anim. s. vert. VI, 1819). C. longa, humilis, supra et infra subparallela, pallida, antice prolongata, sæpissime late rotundata, non angulata, postice plus quam triplo longior, superne angulata, angustata, in rostrum medianum cuneiforme, siepius truncatum, raro paullisper limosum elongata, carina ligamentali distincta, angulata, margine ventrali recto $v$, retuso, postice longe recteque ascendente; umbones tumidi, prominentes; callus marginalis latus, planus. - U. pictorum Auct., nec Linné, qui varietas U. tumidi sit. Cfr. West. Fauna paliarkt. VII, 1890 p. 101.

Typus: c. juvenis rufescenti fulvida, deinde olivaceofulvida vel et præcipue luteoviridis, striis zoniformibus subrugosis fuscis; long. 90, alt. 37, cr. $27 \mathrm{~mm}$. - Rossm. Icon. f. 196.

Forma maxima: c. rugis incrementi dimidii antice fortioribus, dinuidii postici obsoletis; long. 110-115, alt. 40, cr. $30-33 \mathrm{~mm}$.

Forma pumila: long: 70-s0, alt. $30 \mathrm{~mm}$.

Hab. Sueciu in Skåne, Smâland, Westergötland, Östergötland, Nerike, Wermland, Upland et Westmanland. - Dania rarior. Forma maxima: Dania in Sjælland (Damhuussöen). Fennia supra Hallisfors in amne Aura (Mus. Helsingf.).

Var. delphinus Spengl. (Skr. Nat. hist. Selsk. 1793): c. elongato-ovata, levis, nitida, antice circularis, depressa, superne læte viridis, de cætero pallide lutea, umbouibus cinereis, latis altisque, zona fuscobrunnea circumscriptis; long. 50-80 mm. - Dania in Sjalland (in Ladegaardsaaen pr. Kjöbenharn. in amne inter Farnm Sö et Funrsö, Haldsö, Rosborgsö etc.). - Rossm. Icon. f. 71 a. b. jun. 
Var. desteayesi Mich. (Compl. hist. moll. 1831): e. elongato. orata, marginibus subparallelis, ventrali subsinuato, pars posterior altior, 21/4 longior, in rostrum acuminato-truncatum, supra et infra æque subrecte angustata, modice ventricosa, viride lutescens v. brumneo-olivaceolutea, circuitu obscurior, umbones pone marginem anteriorem, forte decorticati, margarita albocoerulea, oleomaculata; long. 70 -75 (Mörch), long. 90, alt. 34, er. $25 \mathrm{~mm}$. (Loc.). - Dania in Tönderkanal et Tingslevsö. - Rossm. Icon. f. 197.

U. limosus Nilss. (Hist. moll. Su. 1822). C. longa, fusca, antice brevis, subtruncato-convexiuscula, depressa et angulata, postice post medium usque plus minusve dilatata, deinde supra et infra æequaliter in rostrum late rotundatum vel truncatum, sxpissime crusta obtectum, longe producta, margine ventr. recto v. curvatulo, postice brevissime ascendente, carina ligamentali obsoleta; umbones lati, rotundati, prominentes; callus marginalis altus, medio obtuse angulatus.

Typus: c. in omnibus atatibus saturate colorata, brunnea seu luteofusca, striis zoniformibus subrugosis saturatius fuscis; long. 85, alt. 35, cr. $25 \mathrm{~mm}$. - Rossm. Icon. f. 199.

Forma maxima: rugre incrementi confertissimie; long. 85 -100, alt. 34-41, er. $28-30 \mathrm{~mm}$.

Forma pumila: long. 39, alt. $18 \mathrm{~mm}$.

Hab. Suecia in provinciis iisdem ac U. rostratus, sed ubique rarior. - Dania in Sjaelland, Fyen et Jylland multis locis. - Fennia ad Impilahti et Sääksmäki in Talkiakoski (Nus. Hel$\operatorname{singf.)}$.

\section{Gen. Margaritana Schum.}

C. crassa, ponderosa, dente cardinali valve dextru. simplici, valuce sinistre bipartito (vel dentibus duobus), ommibus crassissimis, conicis; dentes laterales vel lamelliformes mulli.

M. margaritifera Lin. (Syst. nat. X, 175S, Mya). C. ovali-oblonga, compressiuscula, nigrobrunnea, margine dorsali arcum circuli majoris formante, rentrali medio sinuato, unde concha subreniformis; umbones depressi, siepissime decor- 
ticati vel profunde lateque erosi. Long. 120-135, alt. $50-65$, cr, 35-40 mm. - Rossm. Icon. f. 72, 74 .

Hab. Suecia tota in fluviis et amnibus majoribus, saltem supra circulum polarem usque. - Norvegia tota usque in Ostfinmarken. - Fennia vulgaris.

Tar. elongata (Lam.?) Nilss. (Hist. moll. Su. 1882): c. multo augustior, oblonga, crassa, nigra, rudis, margine superiore postice recto, lente declivo, ventrali minus sinuato, dentes minuti, subconici, crentulati; ex. max. long. 118, alt. 50, cr. $30 \mathrm{~mm}$. - Suecia in rivulis passim (locus orig. Fröllinge in Halland).

Tar. borealis West. (Exposé crit. 1871): c. compressa, medio alta, postice valde producta, ad posteriorem ligamenti partem latissima, area distincta, carina ligamentali lata, margine ventrali subrecto; long. 127 , alt. $52, \mathrm{cr} .34 \mathrm{~mm}$. Suecia in Lule Lappmark (Jockmock, Silbojock) et forma affines in Dalarne (Rönnewallsån par. Wika).

Var. compressa West. (Fauna Suec. 1873): c. compressissima, parte anteriore brevi, rotundato, posteriore late conico, margine dorsali postice forte declivo, ventrali medio retuso, postice subrecto, impressio muscularis postica striis elevatis crebris, dentes minuti, obtusi; long. 120, alt. 60, cr. $30 \mathrm{~mm}$. - Suecia in Dalarne (Utsjövallen par. Ma$\operatorname{lin} g \mathrm{~s})$.

Tar. centricosa West. 1. c.: c. tumida, etiam parte posteriore valde ventricosa, antice et postice rotundata, margine dorsali leviter curvato, postice depresso, ventrali subrecto. medio retusinsculo, dentes acuti; long. 130, alt. 60, cr. 45 mm. - Suecia cum var. compressa.

Var. roissyi Mich. (Compl. hist. nat. 1831): affinis var. elongate, sed parte posteriore conchi longiore, altior et ventricosior, margine dorsali longiore et rectiore, ventrali magis rotundato, vix subsinuato, rostro obtuso et recurvato, dente card. parvo, non crenulato; long. 108, alt. 58, cr. 28 mm. - Suecia (fide clariss. Locard in Contrib. mal. Fr. XIII, 1889).

Gien. Anodonta (Cirv.).

C. sapissime tenuis, fragitis, dentibus nec cardinalibus nec lateralibus instructa (valvis tantummodo ligamento junctis). 
Obs Mirum in modum variat, quoad staturam et faciem, ut quis minus cautus facile crederet se plures habere species, dum unam tenet. Hac verba Linnæi de planta quadam optime in hoc genus incidunt. Tamen confiteor me non audere, experientia hodierna duce, formas mihi cognitas omnes sub species certas, quamque suo loco naturce congruo, collocare. Hoc ut multum est posterorum.

1. Euanodonta Tiest. C. plus minus ovalis, ovata, oblonga vel longe linguaformis et inflata, antice rotundata, dentibus lateralibus vel lamellis nullis.

(1) C. magna, inflata (precipue medio), abbreviata, extus intusque sulcata, late ovata, parte anteriore magno, late rotundato, posteriore $1^{3} / 4$ ad duplo longiore, margine ventrali plus minus arcuato.

A. cygnea Lin. (Syst. nat. X 1758, Mytilus). C. recta, forte convexa, superficie inacpua, olivacea, virescens, fuscozonata, antice alta, rotunda, postice $1^{3 / 4}$ longior, margo dorsalis et ventralis subparalleli, postior rectus, lente descendens, area humilis, non angulata, rostrum perobtusum, submedianum, umbones submediani, parum prominentes. Long. 185, alt. $90, \mathrm{cr}^{2} .60 \mathrm{~mm}$.

Hab. Srecia passim rarior (ex. gr. Småland in Skiresjön gub. Jönköping, Östergötland in urbe Wadstena etc.). - - Dania? - Norvegia? - Fennia in Aland et ad Rautus (Mus. Helsingf.); in lacu Ladoga?

A. eucypha Bourg. (Moll. Aceph. 1881). C. ut illa A. cygnex, sed valde ventricoso-inflata, virescenti-lutea, brunneo- et viride-zonata et tenue denseque radiata, parte anteriore breviore, posteriore $2^{1 / 4}$ longiore, rostro mediano late truncato, margine superiore arcuatulo, ventrali fortius arcuato, posteriore paullo excavato, area fortiore, compressa, angulata. Long. 150 (ant. 55, post. 95), alt. 82, cr. $55 \mathrm{~mm}$. (long. 165, alt. 90 min.: Mörch). - A. cygnea Rossm. Icon. f. 67, Mörch Syn. moll. Dan., »A. cygnea var. straminea Schlüter! Cat. p. 31» (Mörch).

Hab. Srecia in fossa areis in Wadstena. - Dania (ad Jrgerspriis, Broxö, Holsteinborg, Ragnehavesö pr. Ringstad etc.).

Ut varietates $A$. cygneæ suæ enumerat $O$. Nörch sequentes formas mihi ignotas, in Dania collectas: 
"A. lingua Yoldi (Mörch Cat. Toldi, 2, N:o 608): Area radio latissimo convexo circumscripta; long. $148 \mathrm{~mm}$. alt. $79 \mathrm{~mm}$. - Jegerspriis, Krassmose, Bornholm (spec, long. 108 m. alt. 60 m.) Yoldi".

„A. lyngbyana. T. solida, compressa, ovalis, utrinque subuqualiter roturdata, marina, natibus castaneis; rugx incrementi conferti, prominentes; long. $185 \mathrm{~mm}$, alt. $100 \mathrm{~mm}$. - Sorgenfri; spec. minus (long. 149 mm., alt. 72) ab Klaringen ad Söborg Söe (in Museo Chr. VIII).“

A. fragillima Cl. (Chemn. Conch. Cab. 1876). C. sat abbreviata, parum ventrosa, tenuis, interstitiis rugarum incrementi sublevibus, antice rotundata, infra forte declivis, postice plus quam duplo longior, in rostrum breve lateque subsupramedianum truncatum producta; margo superior rectus, elongatus, perparum ascendens, postice (ubi altitudo maxima) angulum magnum cum margine posteriore recto oblique descendente formans, margo inferior medio forte arcuatus, postice valde et subito ascendens; umbones depressæ; margarita sordide coerulescens, maculis magnis oleoflavidis. Long. 110, alt. 64, cr. $30 \mathrm{~mm}$.

Hab. Donic in Lolland (ad Gammeleje: H. L.).

(2) C. magna, ventrosa (jracipue ad umbones), ocali-elongata, parte posteriore 21/2 longiore, margine ventrali elongato-arcuato; umbones depressi.

A. fol'schhammeri Mörch (Syn. moll. Dan. 1864). C. recta, subovali-oblonga, ventricosa, obscure olivaceo-viridis vel-rufescens, zonata, antice altissima, latissima, rotundata, non declivis, postice humilior, in rostrum medianum, mediocre, rotundatum attenuata, margo dorsalis subrectus, posterior subconcavus, ventralis medio coarctatus; area radiis elevatis utrinque binis circumscripta; umbones superne castanei. Long. 190, alt. antice 100, ud finem ligam. 85, cr. $60 \mathrm{~mm}$.

Hab. Dunia in Sjoclland (in piscina ad Donse et ad Hellebek).

A. lirata llörch (Syn. moll. Dan. 1864). C. recta, solidior, ovali-oblonga, ventricosa, viridelutea, rugis incrementi expressis, acutis lirata, interstitiis profundis, angustis, antice rotundato-declivis, postice non humilior, in rostrum sat 
170 C. A. Westerlund, Synopsis Molluscorum Extramarinor. Scandin.

elongatum, medianum, recurvatulum attenuata, margo dorsalis elongato-arcuatus, ventralis late curvatus; umbones depressi. Long. 160, alt. $80 \mathrm{~mm}$. (1. 123, a. 65, cr. 36 mm.: Locard).

Hab. Dania in Sjælland (Classens Have) et Lolland (Iille Kjöbelöv).

A. æneolina Drt. (Journ. Conch. 1893). C. oblonga, parum convexa, tenuis, fragilis, extus intusque sulcato-costata, olivacea, postice in rostrum attenuato-decurvatum vel truncatum, inframedianum producta; margo superior et inferior subparalleli, anteriore rotundato; umbones plicatuli, rubiginosi; crista humilis; impressiones interna superficiales; margarita coeruleo-ineola (infra coerulea, superne maculis srepiss. maximis pallide hepaticis vel æneis), nitida. Long. 72-84, alt. 37-46, cr. 22-26 mm. - (West. in Nachrichtsbl. Mal. Ges. 1894).

Hal. Suecia in Skåne (in Ringsjön ad ostium amnis Rönneholmsin: Hj. W'.).

A. charpyi (Dup.) Bgt. (Moll. Aceph. I, 1881). C. elongato-ovata, non obliqua, rugoso-striata et sulcata, siupe ad maximam partem erosa, fusco-olivacea, v. rufescens, forte et regulariter rentricosa, valvulis non hiantibus, antice rotundatodeclivis, postice $2 \frac{1}{2}$ longior, in rostrum perobtusum medianum producta; margo superior et inferior subaqualiter arcuatuli, subparalleli. Long. 130, alt. 67, cr. $42 \mathrm{~mm}$.

Hab. Dania ad Krjöbenhavn (in Fredriksbergs Have: H. L.).

A. ventricosa C. Pfr. (Naturg. Il. 1825). C. elongato-oblonga, sat oblicua, fusco-virescens, zonis concentricis viridibus $\mathrm{v}$. brunneis, valde ventricosa, antice rotundato-declivis, postice $2 \frac{1}{2}$ longior, in rostrum obtusum medianum producta; margo superior subrectus, posterior concavo-descendens, inferior subrectus, postice magis quam antice arcuatus; margarita coerulescens. Long. 13̄-167, alt. 68-84, cr. $45-58 \mathrm{~mm}$.

Hab. Dania ad Hirtshal (H. L.). 
(3) C. magna. parum ventrosa, longa vel longissima, maryinibus plus minus parallelis rel convexiusculis, parte posteriore $2^{1} / 2-$ $3^{1}$. longiore; area humilis; umbones depressi.

A. sulcata (Lam.) Nilss. (Hist. Moll. Su. 1822). C. recta, ovalioblonga, ventricosa. humilis, olivaceo-viridis, fusca, extus intusque rugata, interstitiis rugarum excavata, antice fere rotundata, postice fere triplo longior, in rostrum longum. medianum, rotundato-truncatum, superne lente, infra celeriter attenuata, margo superior strictus, horizontalis, ventralis vix arcuatulus, postice recte alteque ascendens. Long. $110-150$, alt. $50-70$, $\mathrm{cr}^{2} .30-55 \mathrm{~mm}$. A. cellensis Auct. (non Mytilus zellensis Gimel. $1788=$ Mya arenaria Schröter 1779 , fide E. r. Martens in Nachrichtsbl. Mal. Ges. 1891).

Hab. Suecia meridionalis et media ad Dalarne et Upland. - Dania multis locis. - Fennia in Aland (in palnde ad bolstaholm: Mus. Helsingf.).

A. moulinsiana Dup. (Hist. moll. Fr. 18522). C. ovali-oblonga, parum ventrosa, antice perbrevis, subtruncato-rotundata, postice $3^{3 / 4}$ longior, in rostrum submedianum longum truncato-biangulatum producta, brunneorufescens, margine dorsali leviter arcuato, ventrali subparallelo usque ad finem recto; umbones marg. anterior. valde approximati, depressi, decorticati; area humilis crista vix conspicua. Long. $75-$ 90 , ait. $40-45$, cr. $20-25 \mathrm{~mm}$.

Hab. Suecia in Skane (Ifösjön ad Bomölla: Hj. II.). -Fennia in lacu Lojo in Nyland, in palude Kakskerta et in amme Yläne (Mus. Helsingf.).

A. cariosa Ḱstr. (Mon. in Cihemn. Cab. 1852). C. longa, humilis, lingureformis, olivaceo-brunnea, fusca, postice virideradiata, antice semicircularis, postice $3-3^{1 / 4}$ longior, in rostrum longum, latum, rectum, forte compressum producta, margine dorsali leviter arcuato, ventrali elongatoarcuato, medio siepius impressulo; umbones anteriores, non prominentes, late erosi; margarita alba, oleomaculata. Long. 100-125, alt. $50-58$, cr. $25-35 \mathrm{~mm}$. 
Hab. Suecia in Blekinge (in lacu Mollsjön: Hj. W.), Södermanland (in amne Gudea: Hj. W.) ef ad Stockholm (in Lidingön: Hj. W.).

(4) C. elonyata, medio (margine dorsali ascendente) dilatata, parum ventrosa, area elata, compressa, pars posterior triplo longior, rostro cuneato; umbones depressi.

A. anserirostris Kstr. 1. c. C. irregulariter elongato-ovata, obliqua, brunneo-viridis, superne et ad margines fusca, ad umbones depressos rufescens, rugis numerosis planulatis, antice circulari-rotundata, postice fere quadruplo longior, in rostrum longum, compressum, inframedianum, subrecte truncatum producta, margo superior sat forte ascendens, posterior longe profundeque descendens, ventralis planoconvexus, postice levissime ascendens. Long. 85-110, alt. $50-53$, cr. $30-38 \mathrm{~mm}$.

Hab. Suecia in Östergötland (Kilarpsjön \& Tåkern) et ad Stockholm (Djurgårdsbrunnsviken: Hj. W.). - Dania in Sjælland (in canalibus hortis Frederiksbergensis pr. Kjöbenhavn) et in Hornbacksö (H. L.). A. cellensis var. sulcata Mörch.

A. Iacuum Bourg. (Moll. Aceph. 1881). C. oblonga, virescens, zonis latis obscuris concentricis, ad margines rugosa, parte antica rotundato-descendens, postica $3 \frac{1}{4}$ longior, in rostrum cuneatum, medianum, oblique truncatum producta, margo superior forte ascendens, posterior concavo-descendens, inferior subrectus, postice alte subrecte ascendens. Long. 95, alt. 52, er. $28 \mathrm{~mm}$.

$H a b$. Suecia in Gotland (in Tingstäde träsk: V. L.).

(5) C. magna, solida, ponderosa, ventricosa, ovalis, atrobrunnea vel fuscorirescens. postice duplo longior, maraine dorsali subrecto, parum ascendente; area compressa; umo s tumidi.

A. dupuyi Ray et Drt. (Rev. Zol. 1849). C. obliqua, margine dorsali ascendente, ventricosissima, ad finem ligamenti latissima, rugosa, inferne prasertim profunde sulcata, antice late rotundata, superne angulata, postice in rostrum medianum, truncatum, obtuse biangulatum, supra et infra lineis rectis requaliter attenuata, margine posteriore recto, 
ventrali arcuatulo, postice longe recteque ascendente. Long. 100-120, alt. 55-65, cr. $10-45 \mathrm{~mm}$.

Hab. Dania in ins. Sjælland (in Emdrupsö: H. S.) et Fyen (ad Brahe-Trolleberg: A. F.). - Verosimiliter hue pertinet A. cellensis var. 3. \& minor ( C . incrassata, ventricosa, sordide olivacea, nates sxpe ferruginei, ligamentum validissimum; long. 112 , alt. $60 \mathrm{~mm} . \gg$ ) Mörch in Syn. moll. Danixe (in lacubus Gjentoftesö, Damht'issöen et Bavelsesö inventa).

(6) C. mediocris, tenuis, fragilis, complanata, breve ovalis, luteovirescens, pars postica duplo longior, margo dorsalis subhorizontalis, subrectus, ventralis totus regulariter areuatus; area alta, valde compressa; umbones plane depressi.

A. sturmi Bourg. (Moll. Aceph. I, 1881). C. subrecta, late ovata vel irregulariter rhomboidea, levigata, nitida, luteoviridis, tenue radiata, ad umbones cinerascens vel ferruginea, antice lata, rotundata, superne angulata, margine posteriore recto, celeriter descendente, postice in rostrum breve, latum, medianum compressa, ligamentum fere inconspicuum. Long. $70-90$, alt. 43-59, er. $26 \mathrm{~mm}$. (L. 106, a. $50 \mathrm{~mm}$.: A. cellensis v, intermedia Mörch).

Hab. Suecia in Blekinge (in lacu Tresjön prope Ronneby: Hj. W.) nec non in et cirea Mä̈laren (J. II.). - Dania in Söerne» ad Kjöbenharn, Ladegaardsaaen, Leersöen et Lundehuussöen.

(i) C. late ovalis-subrhombea, ventricosa, antice prolongatr, postice duplo longior, margine dorsali fere recto, ascendente, ventiali plus minus arcuato; area magna, alta, forte angulata; umbones tumidi.

A. piscinalis Nilss. (Hist. Moll. Su. 1822) pr. p. C. crassiuscula, pallide lutea, superne cinerea, natibus rubris, confertim viride radiata, striata et obscurius zonata, antice rotundata, superne angulata, margine posteriore plus minus concavo, ventrali fere regulariter et forte arcuato, postice in rostrum submedianum, subconicum, obtuso-truncatum producta. Long. 100, alt. 60 , cr. $30 \mathrm{~mm}$.

Hab. Srecic a Skảne (locus originalis est Dagstorp in fossis turfosis), ad Stockholm (in Råstadsjön: Hj. IT.). - Dumia 
ad Björnedam, Tranebjerg, Samsö, in piscina ad Skaaninge et stagno turfoso ad Hellebrek (H. L.).

A. opalina Küist. (Mon. in Chemn. Cab. 1852). C. ut piscinalis, sed medio maxime ventricosa, marginibus aculis, compressis, dense striata et plane sulcata, in area et ad oras lamellata, antice late rotundata, pars posterior in rostrum cuneatum latum, truncatum fere, inframedianum, recurvatulum producta, crista sape humilior, margo inferior elongato-curvatus. Long. 100, alt. 67, cr. $30 \mathrm{~mm}$. (125-69 —ú mm.). - (A. radiata v. paludosa Mörch.).

Hab. Dania in Sjiclland (in fossis circa Kjöbenhavn, inter Farum Sö et Fuursö).

Forma subcylindrica, inflata, margine ventrali recto; long. 105, alt. 5 5 mm. - Dania in Fyen (Hofmansgave).

(8) C. late ovata, convexa, antice late semicivalaris, postice in rostrum brece v. brevissimum declive producta, margo superior arcuatus; crista dilatata, rotundata; umbones depressi.

A. retziana Drt. (Journ. Conch. 1893). C. convexo-compressa, sat solida, medio lieviuscula, nitidula, ad oras sulcatula, postice in rostrum brevissimum, surrectum, submedianum, rotundato-acuminata desinens, pallide olivacea vel late viridula, fusco-zonatula, margo superior, anterior et inferior (postice uscque ad medium) forte arcuati. Long. 75-82, alt. $50-55$, cr. $22-25 \mathrm{~mm}$.

Hab. Suecia in maxima meridionali parte prov. Skåne (unde Rossmässler clarissimo Droü̈t olim specinina communicavit), ad Stockholm (in Bredviken, Kumla et Kyrkviken in Lidingön nec non in Djurgårdsbrunnsviken: Hj. W.), in Upland (Långsjön: E. N.). - Dania in Sjæelland (Emdrupsö: H. S.). IVest. in Nachrichtsbl. Mal. (ies. 1894.

A. Ihotellerii Drt. (Journ. Conch. 1892). C. convexa, ad oras striato-lamellosa, nitidula, postice margine longo forte declivi in rostrum breve, obtusum, inframedianum v. subbasale desinens, fusca, vel pallide castanea, ad nates rubiginosa vel decorticata, margo superior valde ascendens, angulo areali alto, inferior leviter arcuatus v. subrectus, 
postice perparum ascendens. Long. 55-70, alt. 40-55, cr. 23-2' mm. (IT.).

Hab. Shecir in Upland (in Långsjön: E. N.).

A. gregalis Drt. (Journ. Conch. 1893). C. arpuiconvexa, rugosula, postice in rostrum breve, attenuato-subacutum, inframedianum desinens, castaneo-rubicata vel brunnea, ad nates rubiginosa, margo superior arcuatus, inferior vix convexus, antice arcuato-ascendens. Long. $80-100$, alt. $45-60$, cr. $30-32 \mathrm{~mm}$. (West. 1. е.).

Hab. Succia Skâne ad Dagstorp (unde Rossmiissler clarissimo Drouët specimina misit), Nermland (ad Carlstad in portu), Giotland (in palude ad Tingstäde: V. L.) et Östergötland (in Ensjön prope Norrköping: J. W.). - Fennia in lacu Lojo in amne Aura ad Hallisfors, in Aland in palude ad Torpby et ad Gorki in flum. Svir: (Mus. Helsingf.).

A. convexa Drt. (Journ. Conch. 1888). C. ovalis, convexa, supra arenata, postice in rostrum breve, attenuato-truncatulum producta, tenuis, sulcatula, olivacea; umbones depressie, plicatule; crista elata; ligamentum breve, tenue. sinu brevi; margarita pallide coerulescens. Long. 62-7.2, alt. $40-44$, cr. $20-26 \mathrm{~mm}$. Juvenis lavis et nitidissima est, pallide flavida, crista virescenti, convexior quam apud species affines in statu juvenili.

Hab. Daniu in lacubus ad Sorö, Esrom et Hvidkilde (H. L.).

(9) C. subovalis-oblonga, antice brevis, postice $3^{\circ}-4^{\circ}$ longini, medio altissima, utrinque subcequaliter in rostrum longum, intramedianum, late truncutum angustata; maryo ventralis rectiusculus.

A. rostrata (Kok.) Rossm. (Icon. f. 284, 1836). C. peroblirqua, subrhombea-oblongata, compressiuscula, fuscula, nigrocingulata, tenuis et fragilis, antice angustata, rotundata, margo dorsalis rectiusculus, valde ascendens, posterior ieque et sensim descendens, ventralis subrectus; area longissima, alta, utrinque sulcis tribus atroviridibus limitata. Long. $75-100$, alt. $43-46$, сr. $25-35 \mathrm{~mm}$. 
Hab. Suecia in Skåne (unde hanc formam accepi, sed locus specialis ignotus est).

A. spuria Yoldi ap. Mörch (Syn. Moll. Danice 1864). C. subovalis, ventricosa, solida, postice humilior, late rostrata; area subcompressa, utrinque canaliculata; margo ligamentalis ascendens, postligamentalis leviter concavus. Long. 105 , alt. $60 \mathrm{~mm}$. Inter A. ponderosam Rossm. Ic. f. 282 et A. rostratam (Kok.) Rossm. Icon. f. 284 intermedia. (Mörch). A helvetica Mörch (fide Bourg.).

Hab. Dania in Bornholm. (Mörch).

A. tremula Drt. (Journ. Conch. 1888). C. ovalis, convexa, supra infraque arcuata, antice late semicircularis, postice in rostrum attenuato-truncatum producta, solida, olivacea, sxpe obsolete viridi-radiata; umbones depressa, plicatula; crista plus minusve elata; margarita polychroa. Long. 90-95, alt. 50-55, cr. $30-32 \mathrm{~mm}$. Juvenis compressa, tenuis, nitida, pallide virescens.

Hab. Dania in amne ad Söborg (H. L.).

A. helvetica Bourg. (Mal. Quater-Cant., 1862). C. oblongo-elongata, parum ventricosa vel compressa, sat tenuis, striis incrementi sulcata, antice fusco-nigrescens, postice sordide luteola, superne late decorticata, antice rotundata, superne angulata, postice in rostrum elongatum, medianum, ascendens producta; margo dorsalis subrectus, leviter lenteque ascendens, e ligamento ad rostri extremitatem hians, margine concaviusculo, margo ventralis arcuatulus, antice ar= cuatus, postice rectus, ascendens; area compressa, humilis, angulo subnullo; umbones depressi. Long. 75 , alt. 40, $\mathrm{cr}^{2}$. 22-26 (100-105 : 45-5' : 25-32 : Bgt.) $\mathrm{mm}$.

Hal. Suecia in Edlösaviken prope Waxholm (R. H.). Celeb. Drouët determinavit.

A. frigida Drt. C. oblongo-elongata, ad umbones sat ventricosa, infra medium forte compressa, tenuis, crebrisulcata, antice castanea vel fusca, postice luteola, antice brevis, rotundata, postice fere triplo longior, in rostrum longum, late 
truncatum inframedianum producta; margo superior arcuatus, inferior totus subrectus, aliquando subretusus; nates depressie (late erose); margarita candidula, livide maculata. Long. 95-110, alt. 50-57, cr. $27-35 \mathrm{~mm}$. (W.). Hab. Sreciu in Jemtland (in Hornsjön ad Brunflo: E. N.)

(10) C. oblongo-ovata, regulariter ventricosa adeoque scepius marginibus obtusis, pars postica $21 / 2-3$ longior, rostro inframediano, recto, late truncata, margine inferiore postice vix vel parum ascendente, crista humili. non angulata; vel rostro submediano, crista altiore et angulata.

A. rossmässleriana Dup. (Ess. moll. Gers 1813). C. sat obliqua, elongato-ovata, sat tenuis, sat ventricosa, ad margines sulcata, castanea, antice declive rotundata, postice plus quam triplo longior, margo superior et posterior fere regulariter in aren longo ad rostrum subbasalem extenso juncti, margo inferior usque ad finem subrectus, antice ascendens; area humilis, crista prominula, non angulata. Long. 100-120, alt, 50-60, cr. $30-40 \mathrm{~mm}$.

Hab. Suecia in Blekinge (in lacu ad Emmahult: $\mathrm{Hj}$. W.) Södermanland (ad Kuddinge: Hj. W.), Stockholm (in Råstadsjön: Hj. W.) et Dalarne (in lacu Hönsan: G. G. A.).-Dania ad Skjoldnesholm (H. L.).

A. Iuxata Held (Isis 1837). C. obliqua, anguste sublanceolata, leviuscula, rufescenti-subnigra, nitidissima, regio umbonalis sordide lutea, antice bene rotundata, sat alta, postice $2^{3 / 4}$ longior, rostro rotundato-truncato, margo superior rectus, posterior subrectus, celeriter descendens, inferior arcuatulus, antice altius ascendens; area humilis, angulata. Long. 83 , alt. 40, cr. $26 \mathrm{~mm}$. (l. 134 , a. $70 \mathrm{~mm} .:$ Mörch). A. cellensis var. 3 llörch.

Hab. Danic in Sjrelland (Hasle, Mose in Amager) et in Bornholm (ad Tönder).

A. inoruata Kstr. (Mon. in Chemn. Cab. 1852). C. obliqua, anguste subelliptica, sat elongata, ventricosa, striatula, an nulis distinctis, nitida, olivaceolutea $\mathrm{r}$. virescens, ad umbones cinerascens, regio umbonalis ferruginea, viride ra- 
diata, antice declive rotundata, postice duplo longior, rostro rotundato-truncato, margo superior arcuatulus, ascendens, posterior subrectus, lente descendens, inferior regulariter extense arcuatus, siepe medio leviter retusus; area modica, angulata. Long. $78-85$, alt. $43-48$, cr. $25-28 \mathrm{~mm}$. (l. 100, a. $53 \mathrm{~mm} .:$ Mörch).

Hab. Suecia in Blekinge (in Tresjön prope Ronneby: Hj. IV.). - Dania in Sjælland (Albertslund ad 'Taastrup, Aerö) et Jylland (Rindsholm ad Viborg).

A. Iævigata Drt. (Journ. Conch. 1893). C. ovata, oblique ventricosa, medio infra medium sat forte compressa, lievigata, rugis incrementi paucis (c. 5), distantibus, fuscis, nitida, antice rufescens, postice virescens vel virescenti-cornea, superne postice radiis duobus fuscis, vel olivacea, postice fusca, umbonibus rufulis, margo superior arcuatus, inferior horizontalis, postice ascendens, in rostrum truncatulum medianum desinens; nates depressæ, area impressa crista media. Long. 70-80, alt. 43-45, cr. 27-30 $\mathrm{mm}$. (West. I. c.)

Hab. Suecia in »Tyska botten» lacus Mälaren* (V. L.), Södermanland (ad Dalbyö: E. N.) et in Ekolssundsriken ad Stockholm (R. H.).

A. nilssoni Kstr. (Mon. in Chemn. Cab. 1852). C. recta, sublanceolato-ovata, modice ventricosa, striatula, rugis paucis obscuris, virescens vel olivaceo-lutescens, ad umbones ferruginea et cinerascens, postice rufescens, radiis duobus latis fuscis, antice sat producta, non angulata, postice in rostrum sat longum, submedianum, latum, oblique truncatum producta, margo superior convexus, posterior abrupte subrecte descendens, inferior fere totus arcuatus, postice subrecte ascendens; area alta, angulata. Long. 106, alt. ว̃5, cr. $35 \mathrm{~mm}$.

Hab. Suecia meridionalis et media passim.

A. baudoniana Drt. (Journ. Conch. 1893). C. ovata, parum convexa, tenuis, brunneo-virescens, postice in rostrum breviter 
truncatum submedianum attenuata, margo dorsalis arcuatus, ventralis arcuatulus, hic postice ascendens; crista longa, prominula; margarita antice albicla, postice coerulescens. Long. 80-100, alt, $45-50$, cr. $23-25 \mathrm{~mm}$. (ITest. 1. c.)

Hub. Suecia in Skảne (ad Hönsinge: S. Nilsson) et Westmanland (in lacu Hellingen: V. L.).

A. fedderseni mh. C. subrhombea, solida, postice late rostrata; margo ventralis antice ascendens, postice rectus; ligamentum validissimum; vestigium distinctum dentium lateralium sub ligamento. Long, 96, alt. $57 \mathrm{~mm}$. (Mörch sub nom. A. incrassatr).

Hab. Dania in Jylland (ad Rindsholm: A. Feddersen).

A. oblonga Mill. (Mém. soc. agr. sc. Angers, 1833)? C. vix obliqua, parum ventrosa, junior vix ventrosula, antice et infra (ubi medio forte) compressa, olivaceobrunnea, rugis levibus postice elatis, postice plus quam duplo longior, in rostrum fere medianum, lente compressum, oblique truncatum, biangulosum elongata, margo superior et inferior leviter et subrequaliter curvati, ille angulo parvo v. obsoleto in marginem posteriorem longum lente declivum transiens, hic medio subretusus, postice subrecte sat alte ad rostrum ascendens; area humilis, longa, umbones rufi, depressi, leviter rugati v. decorticati. Long. 80, alt. 43 , cr. $23 \mathrm{~mm}$.

Hab. Dania in Ladegaardsaaen pr. Kjöbenhavn (A. F.) et ad Haraldsted sö (H. L.)

Cum ullo dubio amiciss Drouët ad hanc speciem specimina mea pro dolor juniora e loco danico citato duxit. Forte majore fide ad $A$. oblongam Mill. pertinent specimina majora (1. 110, a. 5̆, cr. 33 mm.) qua e lacu Tåkern in Östergötland Succir: accepi.

(11) C. obliqua, margine dorsali subrecte et srpius alte ascentente a medio alto utrinque subequaliter attemata, rostrum subme. diamu, late truncatum, margo rentralis vix cureatus, utrinum ascendens, pars posterior $2-2 \frac{1}{2}$ (ravo triplo) lonyior, area com pressa, crista sape alta, angulata. 
A. anatina Lin. (Syst. nat. x. 1758, Mytilus). C. perobliqua, ovali-lanceolata, fragilis, ventrosula vel subcompressa, rugis densis sculpta, sordide olivaceo-virescens, antice depresse rotundata, postice in rostrum breve, submedianum, late conicum, apice angulatum, utrinque lineis rectis subrqualibus attenuata, margo dorsalis subrecte ascendens, posterior sat celeriter descendens, ventralis sæpissime rectus et retusulus, postice oblique ad medium ascendens, area compressa, angulata. Long. 55, alt. 32, cr. $18 \mathrm{~mm}$. (A. anatina v. $\gamma$. Nilsson, A. anatina Rossm. Icon. f. 417).

Hab. Suecia meridionalis et media. - Dania passim. Fennia ad Rantasalmi (Mus. Helsingf.)

A. danica Mörch ap. Bourg. (Moll. Aceph. I. 1881). C. perobliqua, ovato-lanceolata, elongata, compressiuscula, tenuis, antice humillima, parva, rotundata, postice in rostrum latum producta, margo dorsalis rectus, ascendens, ventralis ante medium subretusus, utrinque sensim ascendens; area compressa, medio angulata, utrinque radıis binis subelevatis remotis circumscripta. Long. 90, alt. ad umb. 41 , postligam. 50 , cr. $20 \mathrm{~mm}$.

Hab. Dania multis locis (locus origin. est Hofmansgave in ins. Fyen, ubi frequentissima).

A. pulchella Drt. (Journ. Conch. 1893). C. oblonga, ventrosula, infra medium antice compressa, tenuis at firma, striatosulcatula, pallide castanea, parte posteriore limo calcareo valde adharente forte obtecta, in rostrum latum medianum producta, margo dorsalis arcuatus, ventralis rectiusculus; nates depressæ; crista prominula. Jong. $70-77$, alt 40 -45 , cr. $20-22 \mathrm{~mm}$. (West. l. c.)

Hab. Suecia in Gotland (in palude ad Löjsta: V. L.) et ad Stockholm (in Lidingön: Hj. W.) -- Dania in Haraldsted sö et ad Frederiksdal (H. L.).

A. tenella (Held) Kstr. (Mon. in Chemn. Cab. 1852). C. obliqua, oblonga, convexa, tenuis, marginibus acutis, olivaceo-lutea, arl umbones brunnea, antice prolongata, ovato-rotundata, 
postice duplo longior, rostro sat elongato, late conico, oblicue truncato, infra mediano, margo dorsalis rectus, ascendens, posterior longus, rectus, lente descendens, ventralis elongato-arcuatıs, antice strictiusculus; area hu. milis. Long. $70-80$, alt. $37-40$, cr. $20-24 \mathrm{~mm}$.

Hab. Suecia in Blekinge (in Lângasjö* prope Ronneby: Hj. W.) - Dania in Esrom sö (H. L.).

A. fuliginea Drt. (Journ. Conch. 1893). C. oblonga, supra medium oblique tumida, tenuis, fragilis, fusca, fuliginea, striata, postice in rostrum sat angustum truncatulum producta, margo dorsalis leviter arcuatus, ventralis medio arcuatus. postice longe lenteque ascendens; nates late decorticata fuligineo-brunnea, membranaces, pellucida; margarita coerulescens, livide maculata, polita. Long. 7 4 - 80 , alt. 40 - 1 ó, cr. $25-27 \mathrm{~mm}$. (IVest. 1. с.)

Hab. Suecia in Blekinge (in Långasjö: prope Ronneby: Hj. W., in Södermanland (in Lillsjön ad Dalbyö: E. N). et ad Stockholn (in Lidingön, Kyrkviken: Hj. W.).

A. fennica Drt. C. oblongo-elongatula, subcompressa, tenuis, nitidula, viridis, perfragilis, antice late rotundata, postice duplo longior, in rostrum medianum, surrectum, truncatum producta; margo dorsalis rectiusculus, leviter ascendens, crista elatiore, inferior arcuatus, postice forte ascendens; ligamentum tenue; laminula bene conspicua: margarita coerulea, aneo-maculata. Long. 80, alt. '13́, cr. $32 \mathrm{~mm}$.

Hab. Femiu ad Rimito (E. N.).

A. macella Drt. (Journ. Conch. 1893). C. »oblongo-lanceolata vel subrhombeo-ovata, lata, tumidula, tenuis, fragillima, ad oras striatula, olivacea, antice lata, truncata, postice in rostrum breve, medianum, rotundatum, surrectum producta, margo superior valde ascendens, posterior forte descendens, inferior arcuatulus, postice forte ascendens; crista alta, lata; nates depressa, parum erosa, translucidic, l'ubiginosie; margarita coerulea, livide maculata, lucida. 
Long. 60-67, alt. ad umb. 35, ad cristam 45, cr. 17-18 mm. (West. l. c.)

Hab. Sueria in Blekinge (in Härsjön prope Ronneby: Hj. W.).

A. subluxata Kstr. (Mon. in Chemn. Cab. 1852). C. perobliqua, ovato-elongata, subregulariter ventricosa, marginibus subobtusis, brumneorufa, umbones versus carnea, striata, rugis incrementi numerosis, antice brevissima, angusta, rotundata, postice triplo longior, rostro inframediano subtruncato, margo dorsalis subrecte ascendens, posterior longe recteque descendens, ventralis arcuatulus; area humilis. Long. 80, alt. 43, er. $23 \mathrm{~mm}$.

Hab. Suecia in Blekinge (in Herrstorpsjön prope Ronneby), Södermanland (Drefviken, Tullingesjön et Bredviken: $\mathrm{Hj} . \mathrm{IT}^{\text {.) }}$ et in Mälaren (in »Tyska botten» prope Drottningholm: V. L.). - Dania in Sjælland (Kimmerslövsö prope Ringsted, Hornbæksö, Esromsö) et Jỵlland (in Gudenaa).

A. nycterina Bourg. (Moll. Aceph. I. 1881). C. perobliqua, lanceolata, sat firma, superne ventricosa, virescenti-olivacea, antice bene rotundata, postice $2 \frac{1}{2}$ longior, rostro inframediano, rotundato. margo dorsalis arcuato-ascendens, posterior subconcave declivis, ventralis subrectus; area humilis. Long. 73, alt. 43, cr. $24 \mathrm{~mm}$.

Hab. Suecia in Skåne (ad Kristianstad: Hj. Wr.), Södermanland (Dalbyö: E. N.) et Östergötland (ad Norrköping. J. W.). - Dania in Thorsö ad Silkeborg (A. F.) et in Frederiksdals Aa. (H. L.).

A. westerlundi (Fagot) Bourg. (Moll. Aceph. I. 1881). C. obliqua, oblonga, regulariter ac modice ventricosa, pro magnitudine solida, fusca, grosse striata, antice striata, postice duplo longior, rostro inframediano, rotundato, margo dorsalis vix curvatulus, ascendens, posterior arcuatus et celeriter descendens, ventralis regulariter leviterque curvatus; crista et margo arealis inferior nulli; ligamentum brevissimum. long. 66, alt. 40, cr. $21 \mathrm{~mm}$. 
Ha7. Suecia in Skåne (ad Örtofta).

A. lanceolata Drt. (Journ. Conch. 1893). C. oblongo-lanceolata, superne usque ad rostrum tumidula, infra medium compressa, tenuis, ad oras squamosula, pallide olivacea, postice superne obscura, in rostrum attenuato-truncatum producta, margo superior leviter ascendens, posterior subrectus, sensim descendens, inferior rectiusculus, postice juvenis forte, adultus parum ascendens; nates depressæ (erosa); crista humilis; margarita coerulescens, antice albida. Long. $70-85$, alt. $40-43$, cr. $27-34$ mm. (West. l. c.)

Hal. Suecia in Blekinge (in lacu ad Emmahult*: Hj. W.). - Fennia in lacu Nurmijürvi in Nyland et in Satakunta in amne Yläne (Mus. Helsingf.)

A. limbata Drt. (Journ. Conch. 1893). C. ovata, tumidula, tenuis, lirviuscula, nitida, olivacea, obsolete viride radiata, antice attenuata, postice in rostrum breve, attenuato-obtusum desinens, margo superior longe ascendens, posterior forte descendens, inferior regulariter arcuatus; nates depressie, plicatulæ, rubiginosie; crista elata, dilatata; margarita lactea, peripheria distincte fusco-limbata. Long. 65-80, alt. 40-45, cr. $23 \mathrm{~mm}$. (TTest. l. c.)

Hab. Suecia in Skåne (in amne Keflingeå: R. H.), Westergötland (Brunnsbobäcken" ad Skara: J. W.) et in Mälaren (in Årstaviken: V. L.).

A. minima Nill. (Mem. Soc. agr. d'Angers 1833). C. obliqua, oblongo-ovalis, piceo-nigra, nitida, striatula, zonis paucis, in medio ventrosula, de catero compressa, antice sat depressa, latiuscula, rotundata, postice fere duplo longior, rostro inframediano obtusissimo, margo dorsalis subrectus, ascendens, posterior longus, rectus, ventralis utrinque arcuatus, medio subrectus; umbones forte decorticati, area compressa, medio angulata. Long. 50-60, alt. 35-37, cr. $18-20 \mathrm{~mm}$.

Hab. Suecia in W'estergötland (in Dofsabäcken et Viktoriasjön ad Skara: J. IV.), Östergötland (Kilarpsjön), Södermanland (in Långsjön: Hj. W.) et Upland (Ormgårdssjön: V. L.). 
A. mörchiana Ciless. (Mon. in Chemn. Cab., 1876). C. obliqua, subovalis, brevis, medio ventricosa, luteo-viridis, superne rufescens, area brunnea, antice abbreviata, declive rotundata, postice sesqui longior, rostro breviusculo, submediano, subangusto, apice rotundato, margo dorsalis arcuatulus, posterior subrectus, lente descendens, ventralis arcuatus, utrinque requaliter ascendens; area perangusta, angulo subnullo; ligamentum breve, forte; lunella rotundata. Long. 62, alt. 40, cr. 25 (20) mm.

Hab. Dania in Sjælland (Cilimsö, Tiessö ete.).

A. parvula Drt. (Et. Nay. Fr., 1852). C. elongato-ovalis, obliqua, rentrosula vel subcompressa, brunneo-rufescens (fuscoviridis Loc.), nitida, striatula, lineis incrementi nonnullis $(6-8)$ sat fortibus, antice brevis, angustata, rotundata, margine anteriore forte declivi, superiore arcuato, ascendente, posteriore subrecto, inferiore curvatulo, postice ascendente; pars posterior $2 \frac{1}{2}$ longior, in rostrum late truncatum paullo inframedianum elongata; area compressa, postice angulata, cristata; margarita coerulescens, superne hepatica. Long. $60-70$, alt. $32-40$, cr. 22 (40-30-14: Loc.) $\mathrm{mm}$.

Hab. Suecia in Flatensjön ad Stockholm (Hj. W.). - Dunia in rivulo ad Vigersdal (H. L.).

A. redacta Drt. C. minima, ovata, tenuis, ad marginem striatolamellosa, grisea; margo dorsalis arcuatus, in fine excisus; margo ventralis convexus; margo anticus late semicircularis; pars postica breviuscula, in rostrum breve attenuatosubacutum desinens; crista prominula, incisa; nates depressula, plicato-undatie, rubiginosx; liganentum tenue, breve, obtectum: laminulie lineares, breves; sinus lanceolatus; impressiones superficiales; margarita pallide coerulescens. Long. 60, alt. 39, cr. $20 \mathrm{~mm}$. (Drt.)

Hab. Dania in Bavelsesö et in Glumsö (H. L.). 
(12) C. obliqua, margine dorsali subrecte ascendente, rostrum breve, inframedianm, obtusum, margo ventralis regularitcr curatus. utrinque subuqualiter ascendens, pars posterior 2-21/2 longior, area modica, parva.

A. maculata Shepp. (Trans. Linn. Soc. 1820, Mytilus macula). C. parum obliqua, ovato-rhombea, fere compressa vel regulariter ventrosula, tenuis, rufescenti-viridis, radiata, antice rotundata, postice plus quam $2^{1} / 2$ longior, rostro brevi, inframediano, obtuso, desuper attenuato, margo dorsalis rectiusculus, parum ascendens, posterior concaviusculus, forte et longe descendens, ventralis regulariter arcuatulus, postice leviter ascendens; area sat magna. Long. 62 , alt. 34 , cr. $16 \mathrm{~mm}$. (70-80, 45, 20-24 mm.) Amiciss. O. Mörch in Syn. Moll. Danire sua nt typum hujus speciei figuram 281 superne ad dextrum in Iconographia Rossmässleri citavit et quattuor formas huc pertinentes retulit: 1. C. flavescens radiis obsoletis; 2. C. solida, planior, subunicolor (de qua partim amic. Clessin suam A. mörchianam creavit); 3. C. suborbicularis, complanata, flavescens, unicolor, et 4. C. obscureviridis, natibus ferrugineis, margine ventrali medio subretuso, postice subito ascendente.

Hab. Suecia in Blekinge (in Herrstorpsjön ad Ronneby).

- Dania in Sjæelland, in multis lacubus.

A. sedentaria (Nlab.) Bourg. (Moll. Aceph. I. 1881). C. bene obliqua, ovalis, ventricosa, pricipue superne, sat forte striata, ad oras squamosa, nigricans, olivaceozonata, ad umbones rufa, pars anterior magna, rotundata, infra panllo declivis, postice fere duplo longior, rostro brevi, apice rotundato, inframediano, margo superior rectus, ascendens posterior concaviusculus, ventralis totus regulariter arcuatus; area parva, lunella ligament, maxima. Long. 80, alt. 47, cr. $26 \mathrm{~mm}$. - A. inornata Mörch pr. p.

Hab. Dania in Fyen (in fossa argillacea in Hofmansgave: O. Mörch).

A. incrassata Shepp. (Lin. Trans. 1820). C. elongato-subtriangularis, ventricosa, solida, fuscobrunnea, rugosa, antice 
angustata, rotundata, superne angulata, postice triplo longior, in rostrum subbasale, late truncatum, biangulatum producta, margine conchæ superiore arcuatulo-ascendente, posterior recto-descendente, basali arcuatulo, extenso, umbones depressæ, ligamentum validum. Long. 100, alt. 56, cr. $36 \mathrm{~mm}$.

Hab. Dania ad Kjöbenhavn (in Kastelsgraven: H. L.).

A. avonica (Mont., Test. Brit. 1803) Bgt. C. subquadrangularis, antice angustato-rotundata, postice angusta in rostrum basale obtusum attenuata, margine superiore arcuato, posteriore lente subrecte descendente, basali, antice arcuatc, deinde subrecto, pars posterior $2^{1 / 2}$ longior anteriore, parum ventrosa, sat tenuis. Long. 72, alt. 46, cr. $22 \mathrm{~mm}$. (Mytilus avonensis Mont.)

Hab. Suecia ad Norrköping (J. W.) et ad Dalbyö in pror. Södermanland (E. N.). - Dania in Borup sö et in amne ad Holsteinborg (H. L.).

(13) C. obliqua, margine dorsali et posteriore in arcu longo fere conjunctis, antice anyustata, postice 3-4 longior, rosto seppius inframediano, margo ventralis subrectus, retulosus, crista et angulus fere obliterati.

A. amnica Drt. (Journ. Conch. 1888). C. perobliqua, oblongosubrhombea, elongata, medio subtumida, tenuis, griseolutescens vel pallide castanea, superne post nates fuscoviridis, sublavis et nitida, antice perangusta, declive rotundata, postice triplo vel $4^{\circ}$ longior, rostro submediano, apice rotundato, margo superior brevis, arcuatus, posterior sub. rectus, lente descendens, inferior subrectus, medio retulosus; crista elongata, angulata. Long. 63-70, alt. 31-38, c1. 23-25 $\mathrm{mm}$.

Hab. Suecia in Skåne (Ringsjön in ostio fluminis Rönneholmsån: Hj. $\mathbb{I}^{\top}$.), in Östergötland (Kilarpsjön) et Dalarne (in Husbysjön: C. G.). - Dania ad Varkevads bro et ad Hugeln (H. L.).

A. viridiflava Drt. (Journ. Conch. 1893). C. obliqua, orata, supra medium convexa, infra medium complanata, medio impressa, tenuis, viridiflava, superne post umbones fusco- 
viridis, rugis incrementi concentricis postice et infra densissimis, pars anterior lata, declive rotundata, posterior triplo longior, rosto abbreviato, inframediano, margo dorsalis totus curvatus, rentralis rectiusculus, medio retusus; regio umbonalis late decorticata; area compressa, humilis, vix angulata; ligamentum breve, flavorufum. Long. 70-80, alt. 40-50, er $25 \mathrm{~mm}$. (West. l. e.).

Hab. Fennia in lacu Kallavesi", ad Knopio, et in Satakunta (Mus. Helsingf.).

A. planulata Drt. (Journ. Conch. 1893). C. obliqua, ovalis, supra medium ventrosula, de catero compressa, tenuis, olivacea, dimidio postice fusco-nigra, multoannulata, rugis tenuibus acutis densis, antice brevis, depresso-rotundata, postice triplo longior, rostro inframediano, late truncato, margo dorsalis arcuatus, posterior rectus, longus, lentissime descendens, ventralis rectiusculus, postice paullisper ascendens; umbones depressi, decorticati; area humilis, vix angulosa; ligamentum breve, fuscocastaneum; margarita grosse maculata. Long. 85, alt. 50 , cr. $25 \mathrm{~mm}$.

Hab. Suecia in Jemtland (in Hornsjön ad Brunflo: E. N.) - Fennia in lacn Kallavesi*, ad Kuopio, et in Korpo (Pensar) (Mus. Helsingf.).

(1.i) C. perobliqua, abbreviata et alta, parum ventricosa, antice alta, rotundata, postice perbreris, sesqui longior, celeriter profundeque in rostrum subbasale desinens; margo ventralis totus subrectus; area srepe elata, angulata.

A. callosa (Held) Kstr. (Mon. in Chemn. Cab., 1852). C. oralirhomboidea, solida, striata, rngis pancis, antice olivaceovirescens, postice ferruginea; ligamentum validum, lunella parva; callus marginalis validus, latus. Long. 77, alt. max. 50 , cr. $22 \mathrm{~mm}$. (1. 90, a. 55-60 mm.: Mörch). - A. macula v. 1 Mörch (fide Bourg.).

Hal. Dania in Jỵlland (Aerö, Nonbosö, Viborgsö).

A. orbicularis Drt. C. suborbicularis, requiconvexa; crassula, striatula, lutea, brunneo-zonatula, antice late semicircularis, 
188 C.A. Westerlund, Synopsis Molluscorum Extramarinor. Scandin.

postice in rostrum breve obtusum desinens; margo superior arcuatus, inferior convexus; nates depressæ. parce plicatule (erosi); laminula brevis; margarita argentea, nitida. Long. 77, alt. 55, cr. $30 \mathrm{~mm}$.

Hab. Suecia in prov. Skåne (in Wombsjön: R. H.).

2. Pseudanodonta Bourg. C. ovalis vel rhomboidea, compressissima, antice brevissima, angusta, postice ad angulum carinalem dilatata, deinde subabrupte rostrata, dente anteriore in utraque valva lamelliformi; umbones depressi, natibus parvis, acutis.

A. complanata (Z.) Rossm. (Icon. f. 68, 1835). C. elliptico-ovata, brunneo-ulivacea, nitida, striata, rugis densis, fuscis, margo dorsalis arcuato-ascendens, angulo obtuso in marginem posteriorem longe lenteque declivum et subrectum transiens, margo ventralis extense arcuatus, ad fines sat alte ascendens, postice in rostrum submedianum, triangulareacuminatum desidens. Long. 70, alt. ad umb. 38, a. postligam 40 , cr. $20 \mathrm{~mm}$.

Hab. Suecia in Skåne (Noseå et Almaả), Småland et Östergötland pluribus locis, Lpland, Westmanland (ad Westerås in Svartan), Dalarne (in lacubus Hönsan, Hejan etc.). - Fennia ad Vanajavesi prope Tavastehus, Satakunta, ad Fredrikshamn (in Skogby) et in Aland in palude ad Torpby (Mus. Helsingf.)

A. goësi n. sp. C. a parte anteriore angusta retrorsum sensim et utrinque requaliter marginibus rectis ad tres partes vel ad angulum cristalem sat validum forte dilatata, postice et desuper fortius in rostrum latum, breve et apice subcirculari-rotundatum attenuata. Long. 65 (pars ant. 20, p. post. 45), alt. ad umb. 28, ad ang. 38, cr. $14-15 \mathrm{~mm}$.

Hab. Suecia in Westergötland (in fluvio Lidan : A. G.) et in Östergötland (in amne Svartån: A. G.).

A. klettii Rossm. (Icon. 1. 1835). C. oblongo-elongata, compressa (sæpe convexula), tenuis (sæpe solidula), sulcatula, nitida, virescens vel castanea, antice et postice subrequaliter ro- 
tundalo-obtusa, antice paullo latior, postice in rostrum attenuato-olıtusum medianum producta, margo dorsalis arcuatus, angulo subnullo, ventralis arcuatulus; nates depressæ, erosæ; margarita pallide coerulescens, livide maculata vel albida, siepe incrassata. Long. $75-85$, alt. $37-45$, cr. $16-$ $23 \mathrm{~mm}$.

Maひ. Suecia in Skåne (Kíeflingeå), Östergötland (Skenain et Svartån), Westergötland (in fl. Lidan), Upland (Länna: E. N.) et Dalarne (in lacu Hönsan par. Husby). - Dania in Tiissö, Sorösö et Viborgsö).

Obs. Descriptionem allatam e speciminibns numerosis in lacu Hönsan Dalekarliæe ab amico C. G. Andersson collectis et iis e manu proprio Rossmässleri a loco originali (Grossenhayn Saxoniw) communicatis omnino congruentibus clariss. H. Drouët cum figura correspondente ad dispositionem benigne misit.

A. microptera Borcherd. (Abh. naturw. Bremen, 1888). C. elliptica, antice et postice medio subxqualiter attenuato-angustata et obtusa, margo dorsalis subrecte et alte ascendens, area alta, compressa et alata angulum peracutum cum margine posteriore formans, margo ventralis regulariter arcuatus. Long. 58, alt. ad umb. 24, ad ang. 32, cr. $15 \mathrm{~mm}$.

Hab. Suecia in Östergötland (Kilarpsjön). - Dania in Thorsö ad Silkeborg et in rivulo ad Vigersdal (H. L.).

A. acutalis Drt. (Journ. Conch., 1894). Concha ovalis, supra medium ventrosula, infra medium forte compressa, laviuscula, nitida, viridis vel fusca, postice in rostrum attenuatosubacutum inframedianum vel subbasale desinens, margo dorsalis forte areuatum, ventralis subhorizontalis, subrectus. Long. 50-55, alt, at umb. 25 , medio 33, cr. 13 $-16 \mathrm{~mm}$.

Hab. Suecia in Dalarne (in Brunnsjön: C. G. A.).

A. nordenskiöldi Bourg. (Moll. Aceph. I. 1881). C. longa, elliptica, olivaceo-viridis, rugis fuscis, altitudine maxima accurate in medio marginis superioris, a quo utrinque sque descendens et antice et postice reque angustata et rotundata, margo 
inferior totus regulariter extense arcuatus; pars posterior quadruplo antica longior; area lata, humilis, non angulata. Long. 90 (pars ant. 24, post. 76), alt. ad umb. 40, medio 50 , cr. $18 \mathrm{~mm}$. (l. 65, a. 35 , er. $18 \mathrm{~mm}$. . A. complanata Nord. \& Nyl. Finl. Moll.).

Hab. Snecia in Östergötland (Kisasjön: A. G.). - Fennia in amne ad Frugård in Mäntsälä.

\section{Fam. Dreissenida.}

II. byssifera, pallio antice inferne (pedi) et postice (siptionibus duobus inrequalibus) aperto. C. triquetra, arquivalvis, valde incenulatera, umbonibus anticis, septo transversali interno.

\section{Gen. Dreissena Van Ben.}

\section{D. (polymorpha) fluviatilis Pall. (Voy. en Russie 1771).}

obs. Forme hujus valde variabilis speciei formis intermediis numerosis connexæ sunt. Typus, qualem N. Locard (in monographia generis, Les Dreissensia du système européen, Rev. Suisse de Zool.. T. I. 1893 cum tabulis) desripsit et delineavit, nondum certe apud nos inventus est.

Var, westerlundi Locard 1. c.: c. angusta, altior quam lata, superne fere a medio alto umbones versus oblique descendens, angulo dorsali perdistiucto, mar'gine dorsali leviter, ad rostum forte arcuato; long. 15-19, lat. 7-9, alt. 8-10 mm. - Hab. Dania ad Kjöbenhavn, præcipue in fossis circa urbem. Obs. Suspicor fere hanc cochleam tantummodo juvenem cujusdam, forte sequentis forma, esse.

Var. occidentalis (Bourg.) Loc. 1. c.: c. sat angusta, paullo latior quam alta, antice acuminata, superme longe lenteque ab angulo dorsali patulo declivis, postice longe attenuata, rostro late rotundato, compresso, lateribus leviter arcuatis, margine dorsali primum rectiusculo; long. 33-3í, lat. 17-18, alt. $11-12 \mathrm{~mm}$. (alt. $13-16 \mathrm{~mm}$. f. Loc.). - Dania ad Kjöbenhavn.

Var. locardi (Bourg.) Loc. 1. c.: c. latiuscula rel lata, antice cordato-rotundata, superne ab angulo dorsali distincto forte 
Acta Societatis pro Fama et Flora Fennica, Xlll, n:o 7.

umbones rersus declivis, margine dorsali postice declivi, rostro supra et infia angustato, vix compresso, lateribus arenatis; long. 30-33, lat. 16-18, alt. 13-15 mm. - Dania ad Kjöbenhavn.

Forma ampla mh., concha multo latior quam alta, magna; long. 36-42, lat 23-27, alt. $15-16 \mathrm{~mm}$. - Dania ad - Kjöbenhavn (Österbro.) 


\section{Addenda.}

Pag. 15 .

"Frl. B. Esmark und Stud. med. Z. Aug. Hoyer in Krristiania foederati auctores sunt scripti pro fauna arctica bene meriti »Die Land- und Süsswassermollusken des arktischen Norwegens» (in Malak. Blätter 1886).

Z. Aug. Hoyer etiam scripsit "Lidt om limnxerne» in opere periodico „Naturen", Kristiania 1886 p. 69-74, cum figuris nonnullis bonis.

Pag. 23.

Joh. Tidemand-Ruud annotationes de molluscis norvegicis nec non species plures originales magni pro fauna norvegica ponderis (de quibus infra) benigne mecum communicavit.

Nove forme et nova loca (illie ut hæc e Norvegia fide Birg. Esmark, si non aliter dicitur):

Limax maximus Lin. in Norv. usque ad $66^{\circ} 49^{\prime}$ ascendit.

mut. albus Paasch. -- Norv. ad Bamble; ad Kragerö (Tid.-Ruud).

, mut. fasciatus W. - Norr. ad Laurrik, Modum, Kristiania, Skien.

mut. cinereo-nebulosus Malm. - Norv, etiam ad Laurvik, Malmön et Skien.

Var. cinereus Lister. - Norr. etiam in insulis ad Tromsö et ad Bergen; ad Kragerö (Tid.-Ruud).

Mut. punctatus B. Esm. (J. of Conch. 1886), animal Harescenti-album, fascia lata nigra in cervice, maculis parris albis in medio clypei, postice paullo rariegata. -- Norr., Tromsö prope Areudal.

Var. unicolor Heyn.: animal totum supra nigrescenti-brunneum; - Bergen et Arendal: coerulescenti-griseum; - Bergen: maculis parvis nigris et albis conspersum.

L. marginatus Müll.

Tar. obscurus B. Esm. 1. c.: animal firmum et solidum, non pellucidum, clypeo nigro-brunneo, sape flavido-marginato, 
parte posteriore corporis fusco-flarido $\longleftarrow$ griseo-brunneo. lateribus pallidioribus, carina łavida, raro fascia laterali in clrpeo. - Norr. in Dorre, Ringerige, Laurrik, Kragerö et Lillesand. (B. Esm.) Huc:?

L. agrestis Lin.

Tar. norvegicus W. - Norv. etiam ad Eker.

Titrea crystallina Müll. - Norv. ad Liristiania, Hodum. Romerige, Westfjordalen et Stordöen; ad Kragerö (Tid.-Ruud).

V. contracta W. - Norr. etiam in Forneboskoren, Brdö. Ringerige, Asker, Brevik et Lillesand; Kragerö (Tid.-Ruud).

Zonitoides norvegicus B. Esm. - Norr. etiam ad Fragerö (Tid.-Ruud).

Hyatinia pura Ald. - Norv. etiam ad Eker et Langesund: Kragerö (Tid.-Ruud).

H. Tammonis Str. mut. viridula Ilke. - Torv ad Fagerheim prope Tönsberg.

H. lucida Drap. - Norv. ad Kragerö. (Tid.-Ruud).

H. nitidulu (Drap.??) Rm. - Norv, etiam ad Brgdö et Langesund.

H. cellaria Müll. var. silvatica Mörch (rectius compacta Jeffr. in Brit. Conch. I 1862). - Norv. ad Modum.

Helix aculeata Müll. - Norr. ad Bamble. (Tid.-Puud).

H. lapicida Lin. forma minor. - Norv. ad Bamble prope Langesund (lat. $13^{1 / 2}$, alt. 6 mm.). ad Asker prope Kristiania (lat. $13^{1}, 2$, alt. $4^{1 / 2} \mathrm{~mm}$.).

H. Tispida Lin. ascendit in Norr. saltem ad $62^{\circ}$ lat. bor.

Tar. concinina Jeftr. - Norr. ad Bygdö etc. circa Kristiania et Skien: Kragerö (Tid.-Ruud).

Var. septemtrionalis Cl. - Nor. ad Eker et Langesund.

Tar. depilata C. Pfr. - Norr. etiam ad Sandefjord. Brevik et Skien; Kragerö (Tid.-Ruud) - si vera depilata C. Pfr. et non solum f. detrita rulgaris.

Tar. conica Jeffi. - Norr. ad Brgdö, Eger; Sandefjord et Manger.

H. ericetonm Müll. - Norv. ad Bygdö prope Kiristiania specimen unicum multis retro annis inrentum est, quod in museo zoologico hujus urbis asservatur. Cierte introductum. Ruud).

Pupa cylindracea Da C. - Torv. etiam ad hragerö (Tid.- 
P. muscorum Müll. forma elongata CI. - Norv. ad Trondenæs in Hindö.

P. lilljeborgi W. - Norv, etiam ad Kragerö (Tid.-Ruud).

P. sulstriata Jeffr. - Norv. etiam ad Eker; Kristiansand et Kragerö (Tid.-Ruud).

$P$. (Vertilla) erlandi n. sp. T. circulari-perforata, oblongoconoidea, corneo-brunnea, tenue striatula; anfr. 6, regulariter crescentes, convexi, sut. usque ad aperturam obliqua, ult. callo transversali nullo, levis; apert. sinuato-oralis, pariete perobliquo, lam. 1 parietali intrante, dent. 2 colum. conicis, plicis 3 palat. crassiusculis, longis, medio impressis v. abruptis (1. emersa, sulco extus correspondente, 3. basali), marg. exteriore multo longiore arcus duos formante, superiore fortiore, producto. Long. 2, lat. $1 \mathrm{~mm}$.

Hab. Sueciu ad Dalbỵö. (E. N.).

Succinea putris lin.

Var. limnoidea Pic. - Norv. ad Fragnerdammen prope Kristiania ( 1 specimen lectum 1884 fide B. Esm).

Var. charpentieri Dum. \& Mort. - Norv. ad Brerik (Tid.Ruud).

Var, trianfracta Da C. - Norv, ad Laurvik et Skien.

Var. olivula Baud. (J. Conch. 1877): t. angusta, elongata, S. pfeifferi similis, virescenti-succinea, spira conica, acuta, autr. vix convexiusculi, apert. longa, ovata, sursum acuminata; long, 14-20 mm. - Norv. ad Jarlsberg, Hole et Mjöndalen.

Obs. Clariss. B. Esmark inter species norvegicas hujus generis S. stagnalem Gass. (Mal. de l'Aquit., 1867) in Joum. of Conch. $1886 \mathrm{p}$. 113 nescio quo jure enumeravit, dicens: volour greyish-yellow. Some specimens found in 1870 near Arendal by O. Jensen». Hæc species distinguitur t. elongato-ovata, tenuissima, obsolete costulato-plicata, subænea, virescens, spira brevissima, obtusa, anfr. 3, sutura profunda, apert. magna, columella fere ad apicem a basi intuenti conspicua; long. $41 / 2$, lat. $4 \mathrm{~mm}$ (Gallia).

S. pfeifferi Rossm.

Var. ventricosa Pic. -- Norv. in Gaasö Nordlanden. ఎLong. S, lat. $5 \mathrm{~mm}$.

Var, contorta W. - Norv. in Tromsöen.

Var. propinqua Baud. -- Norv. ad Arendal.

Var. sarsii B. Esm. (Mal. Blätt. 18s6 c. fig.): Duo specimina 
originalia vidi ex quibus t. firma, sat regulariter striata. brunnea, anfr. $3^{1} / 2$. superi convexi, nIt. vix leviter convexus, apert. orata, sursum acuminata. ad basin late rotundata, pariete in spec. minore curratulo, in majore fere in linea recta cum marg. columellari concariusculo; long. $11^{1}, 2$. lat. $6{ }^{1}, 2$, Iong. ap. $7 \mathrm{~mm}$. Figure et descriptio anctoris Esmark a superiore sat aliena non congrumt. - Norr. in ripis flum. Altenelr $\left(70^{\circ}\right)$.

S. agonostoma Kstr. - Norr. etiam ad Sundvolden in Pingerige et in Malmöen.

S. oblonga Drap. forma impura Hn. - Norv. ad Brevik. (Tid.-Ruud).

S. arenaida Bouch. - Norv. etiam ad Langesund.

Limnee stagnalis Lin. var. turgida Mke. - Norv. ad Tönset.

L. anricularia Lin. - Norv. etiam ad Lien, Skien et Krageröd.

"L. mucronata Held. - Nor'r. Vardö in Finmarken. Geh. dickschalig, gelblich weiss, Spindelumschlag fest angedrückt. L. $11^{1} / 2, \mathrm{Br}^{2}$ 10, L. d. Hünd. 10, Br. 7 mm." (B. Esm.).

L. ovata Drap.

Tar. cmpullacea Rossm. forma patulı Da C. - Norr. ad Arendal.

Var. colletti Horer (Mal. BIätt. 1886 e. fig.): t. tenuis, fuscobrunnea, pertenue striata, anfi. 5, convexi, sutura profunda disjuncti, nIt. inflatus, apert. late orata ad rotundata: long. 16-19, lat. 13-14, ap. long. 12--1í, lat. $8-9 \mathrm{~mm}$. -- Norт. ad Laxely in Porsanger. Specimina 3 orig. vidi.

Tar. balthica Lin. - Norr. ad Arendal.

L. peregra Miül.

Tar. ambigua TW. - Norr. ad Jrdderen („verosimiliter"): t. pulcherrima, nitida, tenuissime striatula, flarescenti-brunnea, spira fuscorufobrunnea; long. 17. lat. 9, ap. long. 111, 2. lat. $6^{1}, \mathrm{~mm}$. (B. Esm.).

Tar. peregro-ovata Kob. (Icon. tig. 1489): t. sat tenuis, apert. deorsum et retrorsum producta, spiram multo longitudine superans, precipue margine colum. valde recurro forma insig. nis; ex. fig. Kob. long. 19, lat. 10, ap. long. 17, lat. intra $9 \mathrm{~mm}$. - NorT. ad Salangen in Tromsö Amt et ad histrand in Porsangerfjord. 


\section{L. palustris Müll.}

Var. colombiana Serr. (Mal. Balaton, 1S81): t. non rimata, gracilis et elongata, corneo-castanea, dense tenueque striata, striis spiralibus tenuissimis, spira longa, peracuta, anfi. 7 , perconrexi, sicut tumidi, regulares, ult. convexus. sut. perprofunda, apert. obliqua, semiovata, margine colum. contorto; long. 18 , lat. 6 , apert. Iong. 8 , lat. $4 \mathrm{~mm}$. - Suecia in pror. Östergötland.

Var. septentrionalis Cl. (Verh. Ver. Hamb. 15\%5): t. temis, forte costulata, sæpe rugosa, spira quam apertura paullo longiore, anfr. 7, lente crescentes, sat forte convexi, apert. late orata; long. 16, lat. $7 \mathrm{~mm}$. Cl.) - Norr. in Östensö prope Kristiania, fide B. Esm.

L. truncatula Müll.

Var. microstoma Drt. - Norv. etiam in Helgö in lacu Mjösen, ad Ullensaker, Gudbrandsdalen, Gaasö et Syd-Staranger.

Var. oblonga Puton. - Norr. ad Asker, ad Bryn pr. Kristiania et ad Tromsö; ad Bergen (Tid.-Ruud).

Var. compressa B. Esm. - Norr. etiam ad Statellæ. Brerik et Tromsö.

Planorlis dispar $\mathbf{W}$. - Norv. ad Kragerö. (Tid.-Ruud). Pl. albus Müll. var. hispridus Drap. - Norr. ad Dröbak, Sandvigen in Asker et Bergen; ad Kragerö. (Tid.-Ruud).

Pl. borealis (Lov.) IT.

Var. angigyrus W. - Norv, etiam ad Tönset, Krikne et Ringerige.

Var. chordus n.: t. sub lente regulariter, densissime arguteque striatula, nee non spiraliter lineata et subtus plerumque lineis pancis spiralibus eleratis, anfr. penılt. initio convexus, super ult. eleratus, ult. sæpe medio obsolete angulatus, apert. obliqua, rotundata, marg. callo conjunctis, prarterea ut typus; diam. 6-8 mm. - Norv. ad Fjeldfröskelv in Maalselvdalen, Tromsö Amt. - Pl. borealis B. Esm. in Mal. Blätt. 18s6, a quo specimina 28 originalia ridi, non Pl. borealis WV, in Binn. Conch. V, 1855.

Pl. drapamaldi Sh. - Norv. etiam ad Stavanger, Frederiksstad \&c.

Pl. glaber Jeffr. - Norv. in Gaasö bei Grötö $\left(67^{\circ} 49^{\prime}\right)$ cop.

Bythinia tentaculata Lin. - Norv. in Frognerdammen prope Kristiania. Locus unicus Norvegia apud B. Esm. Valvata pisinalis Müll. - Norv. tota usque ad Pasvigselv. 
Var. discors W. - Norr. ad Ringebo in Gudbrandsclalen. Alt. $4^{7} / 8$, diam. $4^{4 / 5} \mathrm{~mm}$.

V. frigide W. - Norv., Bottneelv in Syd-Stavanger et Salonijavre in Pasvigselv. V. sibirica B. Esm.

T. femica n. sp. T. supra omnino plana, subtus in centro sat angnste at profundissime umbilicata (anfractus inferi in umbilico vix v. angustissime conspicui), anfr. penultimo dilatata, dense striata, nitidula, cornea; anfr. $3 \frac{1}{2} / 2$, celeriter crescentes, ult. validus, dilatatus, cylindraceus, antice non descendens; apert. circularis, omnino horizontalis et omnino extra anfr. penultimum, extus paullo producta, marginibus continuis, supero et infero æequaliter extus illum anfractum areuatis. Diam. $4 \mathrm{~mm}$.

Hab. Fennia ad Vosnessenje prope Onega. (Mus. Helsingf.).

Obs. V. cristatc Miill. proxima species, sed t. multo minor, umbilicus multo latior, anfractus omnes bene prebens, anfr. lentius ac. crescentes, apert. deflexa, margine supero spire parallelo, inferiore multo sub anfr. penultimum arcuato.

Spherium manillanum IV. - Norv. multis locis usque ad $70^{\circ}$ l. b.

\section{Unio trmiches Retz.}

Var. bnrysthenicus (Serv.) Kob. (Ic. f. 1950) aff:: c. margine supero subrecto levissime declivi, inferiore medio leviter curvato, postice alte ascendente, parte conche posteriore quam anteriore triplo longiore in caudam acutam supramedianam producto, c. viridis, radiis fuscis, zonis nigricantibus, umbones erose (typice tuberculis distinctissimis); long. 90, (p. ant. 22, post. 68), alt. 45. cr. $30 \mathrm{~mm}$. - Suecia ad Carlstad (cl. dr. C. Fristedt).

Anorlonta lacum Bgt. - Suec. ad Carlstad. (C. Fr.).

A. anserirostris Küst. - Snec. ad Carlstad. (C. Fr.).

A. tremula Drt. - Suec. ad Carlstad. (C. Fr.).

A. anura n. sp. C. curta, alta, late subrhombeo-ovata, supra medium parum convexa, infra compressa, regione umbonali majore vel minore sublievis, nilida, comea, tota de cæetero densissime tenuissime lamellosa, rarius zonis pluribus obscuris dense lamellosis, interstitiis lievibus flavescentibus; umbones depressie; pars anterior perhumilis, rotundatns, posterior peraltus, 
subabrupte truncatus, margine superiore forte declivi, alea alta, late triangulari, compressa, ligamento brevi, marg. poster. recto, abrupte fere ad basin descendente, cauda basali, brevissima, rotundata, marg. inferiore leviter curvato. Long. 60-65 (parte ant. $20-22$, post. $40-43$, alt. perp. 40--45, max. $46-52$, cr. $26 \mathrm{~mm}$.

Hab. Suec. ad Carlstad (C. F.).

obs. Nomen technicum veterum ,rostrum" pro parte posteriore sæepissime longe producto conchæ valde ineptum videtur, qua causa cilem hoc loco in caudam mutari.

Patula ruderata Stud. - Dan. ad Brónshöjholm in Sjæelland (Helga Rongsted, quæ antea Planorbem cryptomphalum detexit).

Pupa avenacea Brug. - Dan. ad Aakirkeby in Bornholm (Helga Rongsted). (H. S.).

P. lilljeborgi IT. - Dan. ad Kirkerarlöse in Sjalland

Clansitia dubia Drp. - Dan. ad Stege in Bornholm (H. S.). Carychium minimum Nüll.

Var. inflatum Hn. (Pfr. Mon. Auricul., 1856): t. ovata, rentrosula, sp. breve conica, ap. margine exter. forte labiato, obliquo, impresso. - Dan. ad Brónshöjholm in Sjælland (Helga Rongsted). 


\section{Conspectus}

Molluscorum Extramarinorum

\section{Sueciæ, Norvegiæ, Daniæ et Fenniæ}

eorumque destributionis intra Scandinaviam. 1897.

\section{Explicatio Sigiarum:}

D. = Dania, G. = Gothia, S. = Suecia sensu str. (media), S. $b .=$ Suecia superior (Norrland et Lappland), $N .=$ Norvegia, $\boldsymbol{N} . b$. = Norvegia superior (Nordlanden et Finmarken), $F=$ Fennia, $I=$ lslandia.

$\mathrm{Frm} .=$ forma, Var. = varietas; . = raro v. locis perpaucis occurrit, $\ldots=$ parce occurrit, $\ldots=$ late distributa, $-=$ forma plus minusve vulgaris. $X$ ante nomen $=\mathrm{sp}$. adventitia, + ante nomen $=$ sp. subfossiles. 
D. G. S. S.b. N. N.b. F. I.

Cl. 1. Malacosza C'epplalophlora.

Ordo 1. Inoperculata.

Subordo 1. Geophila Fér.

Sect. 1. Monotrema Wr.

Fam. Vitrinidæ.

Subfam. Limacina.

Gen. Limax Müll.

1. Eulimax M. T.

L. maximus Lin.

Nut. niger

albus Paasch

fasciatus

flarescens

flaridus

cinereo-nebulosus Malm

calosoma E. \& St.

leucogaster Mörch .

Tar. cinereus List. .

Mut. punctatus B. Esm.

Var. unicolor Heỹn.

Var. gyratus $\mathrm{IT}$.

Tar. bergensis IT.

2. Malacolimax Malm.

L. tenellus Nilss. .

Mut. phronotus E. \& St. .

3. Clepticolimar (Malm).

L. variegatus Drap.

f. Lehmannia Heyn.

L. marginatus Mïill.

$63^{\circ}$ 


\section{T. S. Sb. I. Li. F. F. I.}

Mut. albomaculatus II. pallens L. E Poll. rupicola L. \& Poll.

Tar. obscurus B. Esm. (Huc?)

Gen. Agriolimax (Mörch).

1. Chorolimax IT.

A. agrestis Lin.

Tar. norregicus $\|^{2}$.

2. Hydrolimax Hẹn.

A. Teydeni Hern.

A. lopris Iü̈ll.

Tar. mucronatus II.

3. Arctolimax IT.

A. Tiyperborens 11 .

Subtam. Titrinina.

Gen. Vitrina Drap.

T. pellucidu Müll. .

Var. dillmrni Jefir.

Var. perforata II.

Tar. brunnensis Llic.

T. angetice Bk.

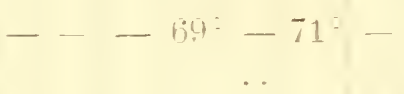

T. holmiensis II.

Subfam. Zonitina.

Cien. Conulus Fitz.

C. Fulune Mïll. .

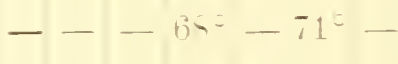

Frm. alderi Grav

Var. mortoni Jeffr.

Var. praticola Reinh.

C. fabricii (Bk.) 


\section{G. S. S J. N. N.b. F. I.}

Gen. Vitrea Fitz.

Y. crystallina Müll. .

Var. subterranea Bgt

V. contracta $\mathrm{W}$.

Gen. Zonitoides Lehm.

Z. vitidus Müll.

Frm. borealis $\mathrm{Cl}$.

Var. parisiacus Mab.

Z. norvegicus B. Esm.

Gen. Hyalinia Ag.

H. pura Ald.

Var. lenticularis Held.

H. hammonis Str.

Mut. viridula Mke.

H. petronella (Ch.) Pfr. .

$\times$ H. lucida Drap.

$\times$ H. villce Strob.

H. cellaria Müll.

Mut. albina.

Var. compacta Jeffr.

H. angulata $\mathrm{W}$.

H. nitidula (Drap.??) Rossm.

H. alliaria Müll.

Mut. viridula Jeffr.

Var. anceps II. .

H. sylvicola Paul. frm. scanica W.

H. upsaliensis $\mathbf{W}$. .

H. nitens Mich.

Mut. helmii Gilb.

Var. olearis $\mathbf{W}$.

Var. lundensis $\mathbf{I V}$. 


\section{Fam. Helicida.}

Subfam. Arionina.

Gen. Arion Fér.

A. ater Lin.

Mut. medius Jens. cinereo-nebulosus Jens. albus Lin.

A. empiricorum Fér.

Mut. mülleri M. T. marginatus N. T.

A. mefus Lin.

Var. lavis IV.

Var. fuscus Müll. .

Var. cinctus Müll.

Var. brunneus Lehm.

A. limacomes $\mathrm{W}$.

A. flavers Nilss.

A. citrimus IV. .

A. nilssoni Poll. "Suecia»

A. hortensis Fér?

A. bourguignati Mab. Destributio?

Subfam. Helicina.

Gien. Punctum Morse.

P. pygmenem Drap.

Gien. Patula Held.

P. rotundata Mïll.

Var. globosa Friedl.

Var. turtoni Flem. .

$P$. ruderata Stud. .

D. G. S. S.b. N. N.b. F. I.

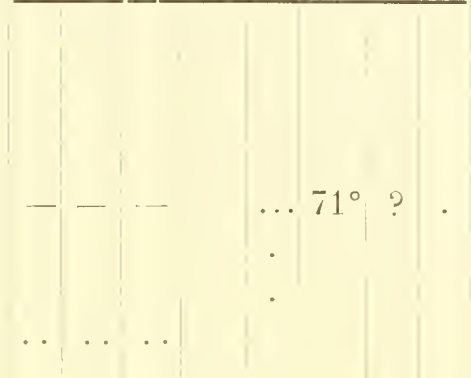

P. strictella Antony.?

$\times P$. rupestris (St.) Drap. 
Gen. Helix Lin.

1. Yallonia Risso.

H. costata Müll.

Frm. cyclostoma IV.

H. pulchella Nüll. $\because H$. adela $\mathrm{IV}$.

2. Acanthinula Bk.

H. lamellata Jeffr.

$H$. aculeata Müill.

Var. sublevis IV.

H. harpe Say

3. Gonostoma Held. (†) H. obvolute Müll. .

4. Chilotrema Leach.

H. Iapicida Lin.

Frm. medelpadensis Cl.

Frm. minor

5. Petasia Bk.

H. bidens Chemn.

6. Trichia Hartm.

H. umbrosa (Partsch)?

H. gyrate IV.

H. hispida lin

Var. concinna Jeffr.

Var. nebula Mke.

Var. septemtrionalis Cl

Var. depilata C. Pfr.

Var. conica Jeffr

Var. sepulcrorum W.

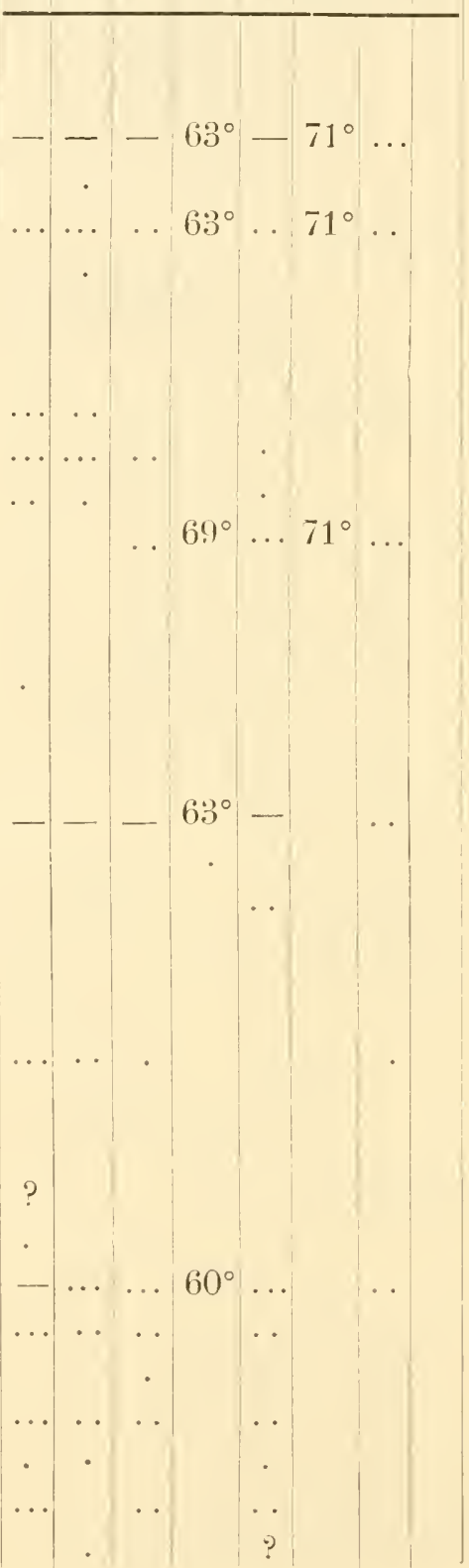


D. G. S. S.b. N. N.b. F. I.

Var. tardigyra $\mathbf{W}$.

Var. mörchi $\mathbb{I}^{\top}$.

H. liberta $\mathrm{WV}$.

H. tumescens $\mathbb{I I}^{-}$.

H. corasia W.

H. rubiginosa (Z.) Schm.

7. Euryomphala II.

H. strigella Drap.

Frm. minor Rossm.

Var. subglobosa IV.

Var. colliniana Bgt.

Subfossilis .

8. Monacha Hartm.

H. incornate Mïll.

Var. creticola (Pouls.) W.

9. Irianta Leach.

H. whustorum Lin.

Frm. minor

Frm. morboso-albina Rm.

Frm. efasciata IV.

Frm. pallida W. .

Frm. lutescens D. \& M.

Var. calcarea Högb.

Frm. creticola Mörch

Var. picea (Z.) Rossm.

Var. septemtrionalis CI.

Var. trochoidalis Roff.

Var. conoidea W'.

Var. alpicola Fér.

Var. rudis Nühlf. alf.

Var. depressa Held 
D. G. S. S.b. T. T.b. F. I.

Var. hypnicola Mab.

Var. thamnivaga Mlab.

Var. foroeli (Bgt.) Serv.

Var. gotlandica W.

Var. oelandica WV.

10. Eulota Hartn.

H. fruticum Müll. .

Frm. minor

Frm. conojdea W.

Frm. andersoni Cl.

Frm. insularum $\mathrm{W}$.

H. scherenlii llidd.

11. Jerophila Held. † Striatella IV.

H. striata Nüll. var. nilssoniana Bk. H. intersecta Poir.

† Helicella M. T. $\times H$. obvia Hartm.

H. ericetorum Müll.

12. Tachea (Leach) Gray. H. nemoralis Müll.

Frm. creticola Miörch

Frm. albolabiata WV.

Var. marginata IV. .

Var. porrecta IV.

H. hortensis Müll. .

Frm. minor Jeffr.

Frm. ludoviciana Drt.

Frm. hybrida Poir.

Frm. crassa $\mathrm{W}$ 


D. G. S. S.l. N. N.b. F. I.

Var. perforata IV.

Var. marginella (C. G. W.) W.

13. Pomatio Bk.

$\times$ H. adspersu Müll.

H. pomatia Lin.

Var. gesneri Hartm.

Subfam. Pupina.

Gen. Buliminus (Ehrbg.) Bk.

B. montanus Drap.

B. olscurtes Mlüll. .

Var. oblongus IV.

Gen. Pupa Drap.

1. Lauria Gray.

P. cylindrace Da C.

Frm. inermis W.

Var. anconostoma (Lowe)

Var. montigena $\mathrm{IV}^{\top}$.

2. Torquilla (Faure-Big.).

$P$. avenucea Brug.

Frm. paucidens $\mathrm{W}$.

Var. subcereana IV.

Var. subhordeum II.

3. Pupilla Leach.

P. muscorum Müll.

Frm. unidentata C. Pfr.

Frm. elongata C.l.

Var. bigranata Rossm.

Var. lundströmi W. 
4. Sphyradium (Ag.).

D. G. S. S.b. N. N.b. F. 1 .

P. edentula Drap. .

Var. turritella IV.

$P$. inomata Mich.

P. columella G. Mts. .

5. Isthmia Gray.

$P$. costulata Nilss.

P. odontostoma $\mathrm{W}$.

P. minutissima Hartm.

6. Hla'a Jeffr.

P. genesii Gredl.

$P$. diccea $\mathbf{I I}$.

$P$. celata $\mathrm{W}$.

P. datiaca $\mathrm{II}$.

Var. dalecarlica II.

P. pineticola $\mathrm{M}$.

P. yemma $\mathrm{IV}$.

P. ovoidea IV.

$P$. arctica Wallenb.

Frm. quadridentata IV.

Var. extima II.

$P$. alpestivs Ald.

Frm. mitis IV.

Frm. septemtrionalis $\mathrm{W}$.

Var. shuttleworthiana (Ch.) .

$P$. eremia IV.

P. romnebyensis $\mathrm{IV}$.

P. lilljeborgi $\mathrm{II}$.

Var. merita IV.

Var. globula IV.

$P$. antivertigo Drap.

Frm. sexdentata Mont.

Frm. octodentata St. 


\section{G. S. S.b. N. N.b. F. I.}

Frm. ferox $\mathrm{II}^{\text {r }}$.

Var. seminulum IV.

P. moulinsiana Dup. .

$P$. gravida $\mathrm{W}$.

P. pygmeea Drap.

Frm. quadridens $\mathbb{W}$.

Var. athesina Gredl.

Frm. bifida $\mathrm{W}^{\top}$.

$P$. substriata Jeffr.

Var. monas II.

7. Vertigo Mïil.

P. pusilla Müll.

*P. tumida W.

$P$. collina $\mathrm{W}$.

P. clevei $\mathrm{W}$.

$P$. crlandi $\mathbf{W}$.

$P$. angustior Jeffr.

Var. producta II'.

Var. gothorum W.

$P$. calliste $\mathrm{W}$.

P. otostoma $\mathrm{IV}^{\mathrm{T}}$.

Gen. Balea Prid.

B. perversa Lin.

Var. suecana IV.

Var. raviana Bgt.

Var. lucifuga (Leach) Bgt.

Var. polita W.

Var. pirostoma II.

Gen. Clausilia Drap.

1. Clausiliastra Möllend.

Cl. laminata Nont. 


\section{G. S. S.b. N. N.b. F. I.}

Frm. minor W.

Frm. castanea WV.

Var. plicifera W.

Var. propinqua W.

Var. pirostoma W.

2. Mlinda Vest.

Cl. biplicata Mont.

Frm. sordida (Z.)

Frm. armata $W$.

Var. austera IV.

Cl. plicata Drp.

Frm. implicata Bz.

Var. elongata A. S.

3. Papillifera (Hn.).

Cl. nilssoni $\mathrm{IV}$.

4. Cusmicia Brus.

Cl. parvula Stud.

Cl. clubia Drap.

Frm. subspeciosa II.

Frm. gracilis $\mathrm{W}$.

Frm. minor IV.

Var. obsoleta A. S.

Var. bifurca W.

Cl. bidentuta Str.

Var. septemtrionalis Schm.

Var. exigua IV.

Var. subrugosa $\mathrm{W}$. .

Var. erronea IV.

Var. retracta W.

Cl. cruciata St. .

Cl. sejuncta $\mathrm{W}$. 


\section{G. S. S.b. N. N.b. F. I.}

Frm. major Schm.

Frm. minor Schm.

Var. truncatula $\mathrm{W}$.

Var. ommæ II.

Var. personata $\mathbb{I}^{+}$.

Cl. comnectens $\mathrm{IV}$.

5. Pirostoma Vest.

Cl. ventricosa Drap.

Frm. tumida Schm.

Frm. gracilior Schm.

Frm. major $\|^{\prime}$.

Cl. rolphi (Leach) Gray.

C7. plicatula Drap.

Frm. curta Schm.

Frm. major 11.

Frm. labiosa Rossm.

Var. fallaciosa $\mathrm{WI}$.

Var. leucostoma IV.

Var. extensa $\mathrm{W}^{\mathrm{T}}$.

Var. scanica II.

Var. oreas IV.

Var. amula $\|^{\top}$.

Var. cruda (Z.) Schm.

Subfam. Stenogyina.

Gen. Cionella Jeffr.

C. (Zua) lubrica Müll.

Frm. nitens (Kok.)

Frm. ovata Jeffr.

Frm. viridula Jeffr. .

Frm. exigua Mke

Var, nilssoni Malm

Tar. collina (Drt.) M. T. . 
Gen. Cæcilianella Bgt.

(. acicula Müll.

Sectio 2. Diplotrema IV.

Fam. Succinidae.

Gien. Succinea Drap.

1. Neritostoma Klein.

S. putris Lin.

Frm. gigantea Bgt.

Var. limnoidea Pic. f.

Var. charpentieri D. \& M.

Var. ferussina M. T.

Var. subglobosa Pascal

Frm. drouetia M. T.

Var. radiata (C. (i. W.) IV.

Var. trianfracta Da C.

Var. temporalis $\mathrm{IV}$.

Var. olivula Baud.

Var. charpyi Baud.

S. parvula Pascal.

S. groenlandica B.

S. stagnalis Gass.?

2. Amphibina Hn.

S. pfeifferi Rossm.

Var. elongata (Hn.)

Var. brevispirata Baud.

Frm. costellata II.

Var. nilssoniana Cl.

Frm. major WV.

Var. mediolanensis Villa

Var. diphya IV.

Var. ventricosa Pic.

Var. propinqua Baud.

Var. elata Baud.

D. G. S. S b. N. N.b. F. I.

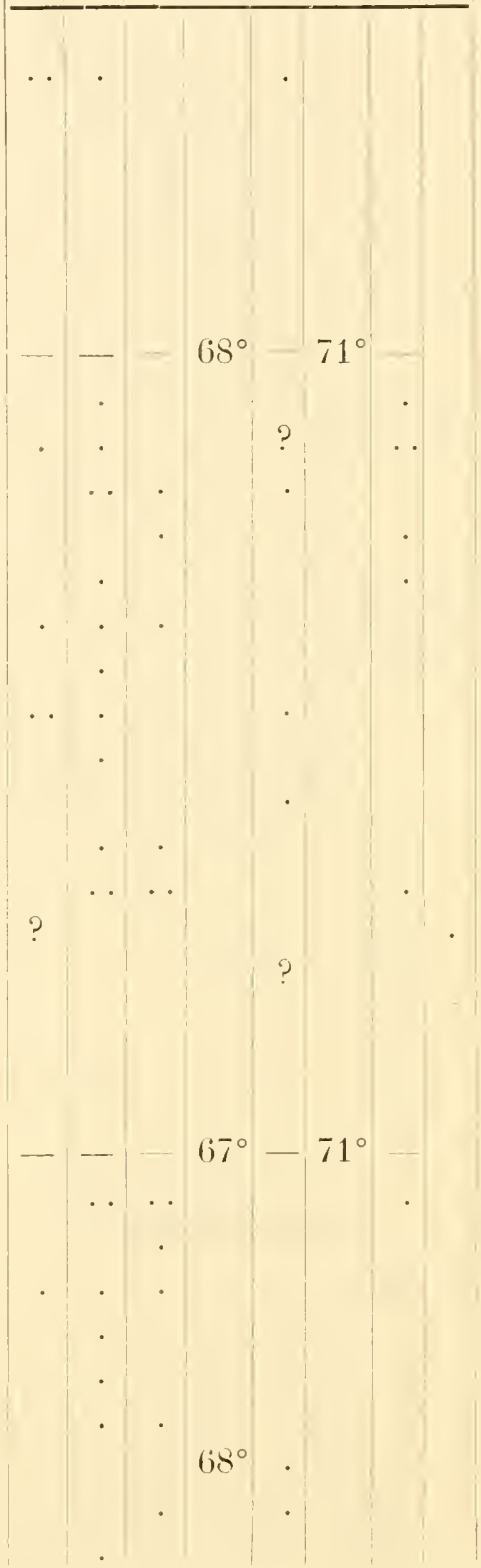




\section{G. S. S.b. N. N.b. F. I.}

Var. contortula Baud.

Var. contorta IV.

Var. gracilis Ald.

Var. subelegans II

Var. sarsi B. Esm.

S. Tente $\mathrm{IT}^{\top}$. .

Frm. de lavali

S. altaica Mts. var. norvegica $\mathbb{W}$.

3. Lucena Oken.

S. oblonga Drap.

Frm. impura (Hn.).

s'. agonostoma Kïst.

*S. lutetiane Mab.

S. borealis $\mathrm{II}$.

S. arenaria Bonch.

Frm. impura $\mathbb{I I}^{\top}$.

Subordo 2. Hygrophila Fér.

Fam. Auriculida.

Gen. Carychium Müll.

C. minimum Nlüll.

Var. inflatum Hn.

Fam. Limnæida.

Subfam. Limnceina.

Gen. Amphipeplea Nilss.

A. glutinosa Müll.

(ien. Limnæa (Brug.) Rang.

1. Lymnus Montf.

L. stagnalis Lin. 
D. G. S S.b. N. N.b. F.

Tar. sophronia II.

Var. westerlundi Loc. .

Frm. neréni II.

Var. colpodia Bgt.

Var. gotlandica IV.

Frm. diffusa II.

Frm. retusa $\mathrm{IT}^{ }$.

Var. elegans (Leach)

Var. roseolabiata (ITolf)

Var. torsa II.

Var. turgida Mke.

Var. borealis Bgt

Var. lacustris (Stud.)

Var. nordmanni II'.

Var. media Hartm.

Var. alata IV.

Var. bottnica Cl.

Var. baltica Lindstr.

Var. livonica Kob.

\section{Gulnaria Leach.}

L. auricularia Lin.

Var. ventricosa Hartm.

Var. concilians $\mathbb{I V}$.

Var. canalis (Villa)

Var. elongata Kob.

*L. rmpla Hartm. .

Frm. pumila Mörch

Var. plicata WV. .

Var. monnardi Hartm.

L. mucronata Held?

Var. alpestris C.I.

L. lagotis Schr.

Var. diploa IV. 
Var. alata Sporl.

Var. anderssoni Cl.

Var. margaritacea $I^{\text {. }}$.

Var. auricula $\mathrm{II}^{\mathrm{T}}$.

广L. prisca W.

L. ampulla Küst. form. lavis $\mathrm{IT}^{\top}$.

L. ovata Drap. .

Var. intermedia Mich.

Var. surcula $W^{T}$.

Var. fontinalis (St.) f. fennica $\Pi$. .

Var. vulgaris C. Pfr.

Var. sericina (C. G. W.) WT.

Var. amnicola (C. G. W.) W.

Var. subinflata $\mathrm{IV}$.

Var. raboti II.

Var. lindströmi $W$.

Var. obtusa Kob.

Var. ampullacea $\mathrm{Rm}$.

Frm. patula Da C.

Frm. lacustris Leach?. ?

Frm. hemispherica $k$.

Var. bulla IV.

Var. colletti Hoy

D. G. S. S.b. N. N.b. F. I.

Var. balthica Lin.

Frm. prævalens $\mathrm{W}$.

Var. succinea Nilss.

Var. steenstrupi Cl.

L. peregra Müll.

Frm. labiata Rm.

Var. paludinoides Mörch

Var. ambigua $\mathbf{I}$.

Var. peregro-ovata Kob.

Var. maritima Jeffr. 


\section{G. S. S.b. X. X.b. F. I.}

Frm. contigua 11 . . . Var. meta II.

Frm. firma IT.

Var. petronia $\mathbb{I T}$.

Var. alpicola IV.

Var. curta Cl.

Var. atrata Chemn.

Tar. orthopieura (C. G. IT.) IT.

Var. decollata (Anders.) $\mathrm{IT}^{*}$.

Tar. geisericola Bk.

\section{Limnophysa Fitz.}

L. palustris Müll.

Tar. fusca C. Pfr.

Tar. septemtrionalis $\mathrm{Cl}$.

Var. flarida Cl.

Var. corrus Gm.

Var. corviformis (Bgt.)

Var. litoralis $\mathrm{TI}$.

Var. stenostoma IT

Tar. turricula Held

Var. colombiana Serr.

\section{Leptolimncea Sw.}

L. glabra Müill.

Tar. labiata II.

Tar. elongata (Jeffr.?) W.

Tar. clarata II.

\section{Fossaria II.}

L. truncatula Miuill.

Var. labiata II.

Var. mörchi II.

Var. gothica 11 . 
I. G. $\sum \sum N Y M=1$

Var. major M. T.

Var. maximella Cob

Tar. microztoma Dis.

Var. goupli I. I.

Var. Echneideri B. Esm. .

Tar. oblonga Put.

Tar. compressa B. Esm. .

Var. lapponica $T^{\prime}$.

Tar. minima Colb.

Frm. gracilis $\mathbb{T}^{-}$.

Subtam. Plivint

Gen. Physa Drap.

1. Bulinus Adar.

Pr. Fontinatis Lin.

Frm. normalis.

Frmi. trpica

Tar. bulla Mall.

Frm. arandis II

Var. oblonga II. $P$ ? . smiglobos $\mathrm{II}^{\circ}$.

2. Inuta Leach.

P7. Then numens Lin.

Mut, albe=cens M[orkh

Frm. achatina lim.

Frm. minor

Tar. perissiana Dup.

subram. Plunotina.

Gen. Planorbis Guett.

1. Girodistze Gtein.

Pl. comene Lin.

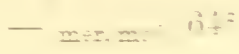




\section{G. S. S.b. N. N.b. F. I.}

Pl. elopthitus Bgt. var. ammonoceras $\mathrm{W}$.

Pl. cryptomphalus IV.

2. Tropidiscus Stein.

Pl. umbilicatus Nüll. .

Frm. filocincta $\mathbb{W}$.

Frm. ecarinata $\mathrm{IT}^{\top}$.

Frm. labiata II. .

Frm. catina $\mathrm{II}^{\mathrm{T}}$.

Pl. carinatus Müll.

Frm. nummularis Mörch

Var. disciformis Jeffr.

Var. turgidus IV.

Var. dubius Hartm.

3. Diplodiscus $\mathrm{IT}$.

Pl. vortex Lin. .

Var. complessus Mich.

Var. discoides Reinh.

Var. discus (Parr.) Rm.

Var. goësi II.

Var. poulseni IV.

Pl. vorticulus Trosch.

$P l$. charteus Held.

Pl. spirorbis (Lin.?) Müll.

Frm. major $\mathrm{II}^{\prime}$.

Var. lencostoma (Poir.) Mill.

Frm. inajor $\mathrm{WT}$.

Frm. perizii (Graëlls) Pl. dazuvi Mörch.

4. Bathyomphalus Ag. Pl. contortus lin. . 
D. G. S. S.b. N. N.b. F. I.

Frm. major $\mathrm{W}$.

Frm. labiata $\mathrm{W}$.

Var. difformis 11 .

Pl. dispar $\mathrm{WT}^{\mathrm{T}}$

5. Gyraulus Ag.

Pl. albus Nï̈ll. .

Var. hispidus Drap.

Var. cinctutus IV.

Var. alatus W.

Var. spurius IV.

Pl. socires II.

Pl. arcticus Bk.

$P l$. strömi $\mathbb{W}$.

Pl. borealis (Lovén) $\mathrm{IT}$.

Frm. minor IV.

Var. angigyrus $\mathrm{IV}^{\mathrm{T}}$.

Var. frigidus WI.

Var, deflexus II.

Var. chordus II.

Il. gredleri (Bz.) Gredl.

$P l$. glaber Jeffr.

Pl. diaparnaldi Sh.

Pl. limoplitus IV. .

$P l$. concinnus $I V$.

Var. gothicus WV.

$P l$. correctus $\mathrm{II}^{\top}$.

$P l$. cochlearis $\mathrm{II}$.

Pl. gelidus $\mathrm{W}$.

6. Armiger Hartm.

Pl. crista Lin.

Var. imbricatus Müll.

$P l$. nautilens Lin. . 
D. G. S. S.b. N. N.b. F. I.

7. Hippentis $\mathrm{Ag}$.

Pl. fontanus Lightf.

Var. eupheus Bgt.

Pl. riparius II.

Pl. leptostoma $\mathrm{II}$. .

8. Segmentinus Flem. Pl. nitidus Müill.

Var. clessini W.

Var. oelandicus IV.

Subfam. Ancylina.

Gen. Ancylus Gieoffr.

1. Ancylastrum M. T.

A. simplex Buc'hoz.

Val. cornu Cl.

Var. westerlundi Cl.

Var. subcircularis Cl.

Var. gibbosus Bgt.

A. danicus IV.

2. Velletia Gray.

A. lacustris Lin.

\section{Ordo 2. Operculata.}

Subordo 1. Geophila.

Fam. Cyclostomidae.

Subfam. Truncatellina.

Gen. Acme Hartm.

A. polita Hartm.

A. lineata Drap.

Gen. Cyclostoma Iam.

C. elegans Müill. 
D. G. S. S.b. N. N.b. F. I.

Subordo 2. Hygrophila.

Fam. Paludinida.

Gien. Paludina Lam.

P. contecta Mill.

Var. lacustris Bk.

Var. paludosa Bgt.

$P$. gallandi Bgt. var. occidentelis Bgt.

$P$. vivipara Lin.

Var. nevilli Bgt. aff.

Fam. Bythinidae.

Cien. Bythinia Leach.

B. tentaculata Lin.

Frm. gigas Mörch.

Frm. intricata $\mathrm{II}$.

Frm. gemina $\mathbb{V}^{\mathrm{T}}$.

Var. producta Mlke.

Var. ventricosa Mke.

Var. bottnica (Anders.) Cl.

Var. sebethina Cout.

Var. michaudi Duv.

Frin. excavata Jeffr.

Var. decipiens Mill.

Frm. opportuna $\mathrm{W}$.

Var. soluta IV.

B. leachii Sh.

Var. inflata Hans.

Fam. Rissoida.

Subfam. Hydrobiina.

Gen. Hydrobia Hartm.

H. steini Mlts.

Var. scholtzi Schm. 


\section{Fam. Valratidx.}

D. G. S. S.b. N. N.b.:F: l.

Gen. Valvata Mïill.

1. Concinna Hübn.

V. antiqua Sow.

*V. pusilla Müll.

$V$. lilljeborgi W.

$V$. piscinalis Müll.

Var. borealis Milach.

Var. discors II. .

Var. ambigua W.

V. cyclostoma $\mathrm{W}$.

Frm. halopea W.

$V$. alpestris (Blaun.) Küst. $\div V$. glacialis $\mathrm{W}$.

T. raboti W.

\section{Tropidina Ad.}

V. nana IV.

V. macrostoma Steenb.

Frm. malleata $\mathrm{W}$.

Var. umbilicata (Fitz.) W.

Var. pulchella (Stud.) WV.

V. frigida $\mathrm{IV}$.

3. Gyrorbis Fitz.

Y. cristata Müll.

$V$. fennica $\mathrm{W}$. .

Fam. Neritinida.

Gen. Neritina Lam.

N. Huniatilis Lin. .

Var. fontinalis Brard

Var. lacustris Lin.

Var. spirata W. .

Frm. minor. 
D. G. S. S.b. N. N.b. F. I.

Var. litoralis Lin.

Frm. baltica (Bk.)

C.. II. Malacozoa Acephala.

Fam. Sphreriida.

Gen. Sphærium Scop.

1. Sphariastrum Bgt.

S. rivicola (Leach) Lam.

2. Cyrenastrum Bgt. \% s. subsolidum Cil.

3. Corneola Ci.

S. corneum Lin.

Var. nucleus Stud. .

Frm. orcula W.

Var. firmum (Cl.) W. . . . .

S. ulicnyi $\mathrm{IT}$.

S. ovale Fér.

S. manillanum $\mathrm{IV}$.

Var. tomentosum W.

Var. suecicum Cl.

S. dupplicatum C.I.

S. vestertundi (Cil.) W.

S. physale W.

4. Calyculina Ci.

S. lacustre Miill.

Var. mamillare Gass.

S. pitace $\mathrm{IV}$.

S. brochoniamm Bgt.

Var. steini Schm. 
D. G. S. S.b.|N. N.b. F. $\mid$ I.

S. ryckholti Norm. var. danicum Cl.

Frm. major $\mathrm{W}$.

Var. strictum (Norm.) Cl.

Gen. Pisidium C. Pfr.

1. Fluminina $\mathrm{Cl}$.

P. amnicum Nlüll. .

Var. glaciale IV.

Var. elongatum Baud.

Var. malmi (Cl.) IV.

Var. striolatum Baud.

2. Tivivulina Cil.

P. supinum Schm.

P. giobulare (Cil.) $\mathrm{IV}$.

3. Fossarina Cl.

$P$. henslowianum Sh.

Var. suecicum (Cl.) IV.

P. parvulum (C.I.) WT.

Var. martensi (Cil.) $\mathrm{W}$.

P. pulchellum Jen.

$P$. nitidum Jen.

Var. fedderseni $\mathbf{W}$.

P. lilljeborgi Cil.

Var. transversale Ci.

Var. hoyeri Cl.

广P. lindströmi Cl.

$P$. fontinale C. Pfr.

Var. acuminatum (CI.) $\mathrm{W}$.

Var. flavescens Cl.

$P$. cinereum Ald.

$P$. pallidum Gass.

P. lovéni Cl. 
D. G. S. S.b. N. N.b. F. I.

P. arcticum $\mathrm{IT}^{\mathrm{T}}$.

P. subtruncatum Malm

Var. sphenoides IV.

Var. poulseni Cl.

P. scholtzi Cl.

Var. lapponicum (Cl.) IV.

P. milium Held.

Var. oidaleum C. G. II. .

Var. unioides W.

P. obtusale (Lam.?) C. Pfr.

Var. magnificum (CI.) W.

Var. fragile (Cl.) $\mathrm{W}$.

Var. solidum (Ci.) W.

Var. esmarkianum Cil.

* P. personatum Malm

P. pusillum Gm.

Var. feröense Mörch

$70^{\circ}$

\section{Fam. Unionida.}

Gen. Unio Retz.

$U$. crassus Retz.

Var. acutus Rossm.

Var. rubens Mke.

$U$. barys $\mathrm{W}$.

$U$. ater Nilss.

Var. subdecurvatus W.

Var. dannemoræe (Mörch) Bgt.

Var. septemtrionalis (Bgt.)

U. elegans $\mathrm{IT}$.

$L^{\top}$. batavus M. \& R. .

Frm. minor $\mathrm{II}$.

U. tumidus Retz.

Frm. major.

Var. mülleri Rossm. 
Frm. compressa Mörch

Var. ovalis Mont.

Var. borysthenicus (Serv.) .

Var. lacustris Rossm.

Var. limicola Mörch

Frm. elongata $\mathrm{IV}^{\top}$.

Var. conus Spengl.

Var. pictus Bk.

Var. tumidulus Loc.

Var. saccatus Rossm.

$U$. rostratus Lam.

Frm. maxima.

Frm. pumila.

Var. delphinus Speng].

Var. deshayesi Mich.

$U$. limosus Nilss.

Frm. maxima.

Frm. pumila.

Gen. Margaritana Schm.

M. margaritifera Lin.

Var. elongata (Lam.?) Nilss.

Var. borealis W.

Var. compressa W'

Var. ventricosa $W$.

Var. roissyi Mich.

Gen. Anodonta (Cuv.).

1. Euanodonta IV.

A. cygnea Lin. .

A. encypha Bgt.

A. lingua (Yoldi) Mörch .

A. lyngbyana Mörch

A. fragitissima CI. 
D. G. S. S.b. N. N.b. F. I.

A. forschhammeri Mörch

A. lirata Mörch

A. aneolina Drt.

A. charpyi (Dup.) Bgt.

A. ventricosa C. Pfr. .

A. sulcata (Lam.) Nilss.

A. moulinsiana Dup. .

A. cariosa Kï̈st.

A. anserirostris Küst.

A. lacum Bgt.

A. dupyi Ray et Drt.

A. sturmi Bgt.

A. piscinalis Nilss.

A. opalina Küst.

Frm. subcylindrica $W$.

A. retziana Drt.

A. Thotellerii Drt.

A. gregalis Drt.

A. convexa Drt.

A. tremula Drt.

A. vostrata (Kok.) Rossm.

A. spuria (Yoldi) Mörch

A. helvetica Bgt.

A. frigida Drt. .

A. rossmässteriana Dup.

1. luxatu Held

A. inornata Küst.

A. levigata Drt.

A. nilssoni Küst.

A. baudoniana Dit.

A. feclderseni IV.

A. oblonga Mill.

A. anatina Lin.

A. danica (Mörch) Bgt. . 
D. G. S. S.b. N. $\mid$ N.b. $\mid$ F. $/$ I.

A. pulchellu Drt.

A. tenella (Held) Küst.

A. fuliginea Drt.

A. fennica Drt.

A. macella Drt.

A. subluxata Küst.

A. nycterina Bgt.

A. vesterlundi (Fag.) Bgt.

A. lanceolata Drt.

A. limbata Drt. .

A. minima Mill.

A. mörchiana Cl.

A. parvula Drt.

A. redacta Drt.

A. macula Sh.

A. sedentaria (Mab.) Bgt.

A. incrassata Sh.

A. avonica (Mont.) Bgt.

A. amnica Drt. .

A. viridiflava Drt.

A. planulata Drt.

A. callosa (Held) Küst.

A. orbicularis Drt.

A. anura $\mathrm{W}$.

\section{Pseudanodonta Bgt.}

A. complanata (Z.) Rim.

A. goësi W. .

A. lilettii $\mathrm{Rm}$.

A. microptera Bocherd.

A. acutalis Drt.

A. nordenskiöldi Bgt. 


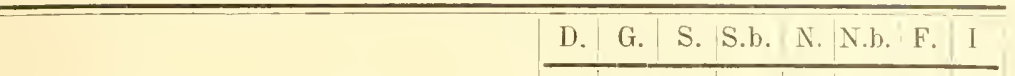

Fam. Dreissenida.

Genus Dreissena Van Ben.

D. fuviatilis Pall.

Var. locardi (Bgt.) Loc.

Frm. ampla W.

Var. westerlundi Loe.

Var. occidentalis Loc. 
Summa molluscorum extramarinorum Scandinaviæ.

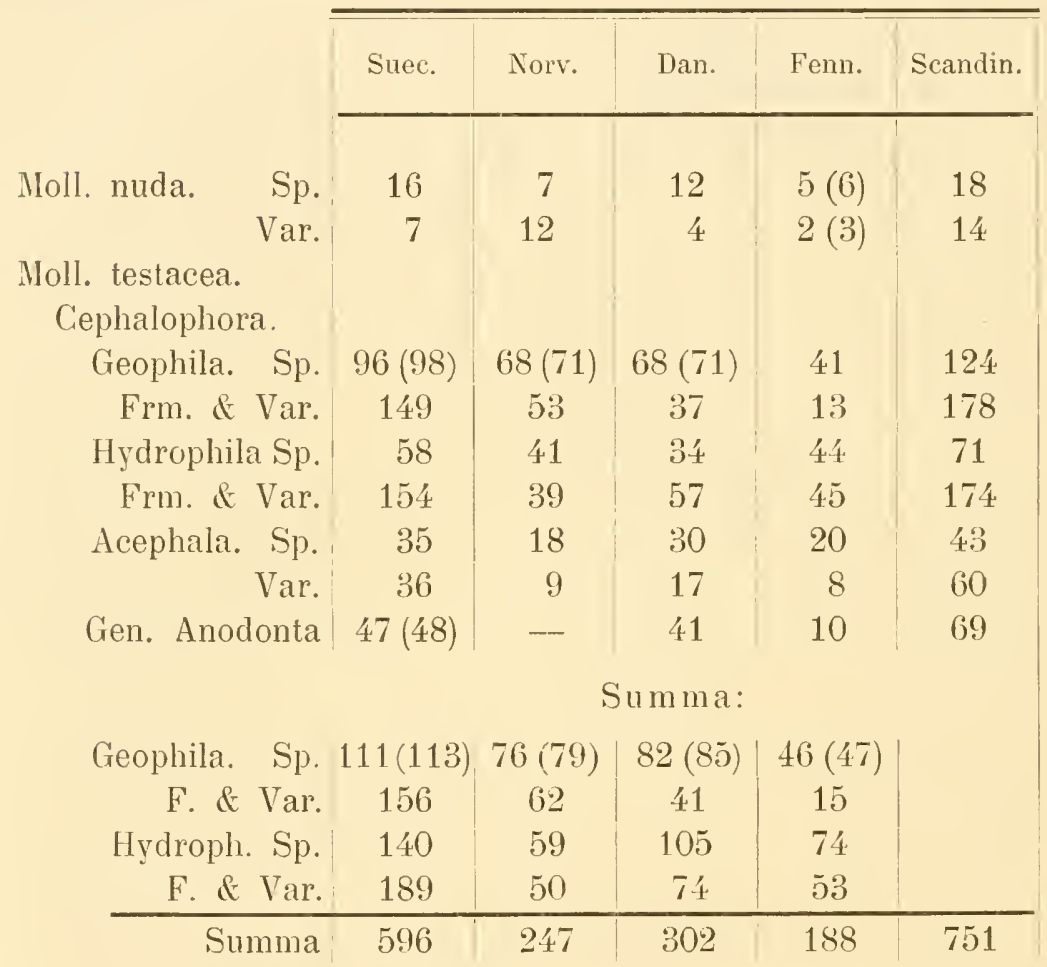




\section{Index.}

Acanthinula Bk. . . . . . 45

Acme lineata Drp. . . . . 129 polita Hn. . . . . . 129

Agriolimax agrestis L. . . . 29

heydeni Heyn. . 30

hyperboreus T. . 30

lævis Miill. . . 30

mucronatus W. . 30

norvegicus W. . 30

Alæa" Jeffr. . . . . 63

Amphibina (Hn.) . . . . . 85

Alinda Vest . . . . . 75

Amphipeplea glutinosa Müll. . 90

Ancylastrum M. T. . . . . 127

Ancylus cornu Cl. . . . . 128

danicus W. . . . $12 \mathrm{~S}$

gibbosus B. . . . 128

lacustris $\mathrm{L}$. . . . 128

simplex Buc'h. . . . 127

subcircularis $\mathrm{Cl}$. . 128

westerlundi $\mathrm{Cl}$. . . 128

Anodonta acutalis Drt. . . . 189

æneolina Drt. . . 170

amnica Drt. . . 186

anatina L. . . . 180

anserirostris Kstr.. 172

anura W. . . . . 197

avonica (Mont.) B. 186

baudoniana Drt. . 178

callosa Kstr. . . 187

cariosa Kstr. . . 171

charpyi B. . . . 170

complanata Rm. . 188

convexa Drt. . . 175

cygnæa L. . . . 168

danica B. . . . 180

dupuvi R. \& Drt. . 172

eucypha B. . . . 168

fedderseni H. . . 179

fennica Drt. . . . 181

forschhammeri

Mörch . . . 169

fragillima $\mathrm{Cl}$. . 169

frigida Drt. . . . 176

fuliginea Drt. . . 181
Pag.

Anodonta goësi W. . . . . 188

gregalis Drt. . . . 175

helvetica B. . . 176

incrassata Sh. . . 185

inornata Kstr. . . 177

klettii Rm. . . 188

lacuum B. . . . . 172

lævigata Drt. . . 178

lanceolata Drt. . . 183

lhotellerii Drt. . . 174

limbata Drt. . . . 183

lingua Mörch . . 169

lirata Mörch . . . 169

luxata Held . . 177

lyngbyana Mörch . 169

macella Drt. . . . 181

maculata Sh.. . . 185

microptera Borch. . 189

minima Mill. . . 183

moulinsiana Dup. . 171

mörchiana $\mathrm{Cl}$. . . 18 í

nilssoni Kstr. . . 178

nordenskiöldi B. . 189

- nycterina B. . . . 182

oblonga Mill. . . 179

opalina Kstr. . . 174

orbicularis Drt. . 187

parvula Sh. . . 184

piscinalis Nilss. . 173

planulata Drt. . . 187

pulchella Drt. . . 180

redacta Drt. . . . 181

retziana Drt. . . . 174

rossmässleriana

Dup. . . . . 177

rostrata Rm. . . 175

sedentaria B. . . 185

spuria Mörch . . 176

sturmi B. . . . 173

subcylindrica W. . 174

subluxata Kstr.. . 18?

sulcata (Lam.)

Nilss. . . . 171

tenella Kstr. . . . 180

tremula Drt. . . 176 


\begin{tabular}{|c|c|c|c|c|c|c|}
\hline & & Pag. & & & & Pag. \\
\hline Anodonta ventricosa C. P & & 170 & Chilotren & ma Leach & . & 46 \\
\hline ,, viridiflava Drt. & & 186 & Chorolim & $\max W$. & . & 29 \\
\hline westerlundi B. & & 182 & Cincinna & a Hübn. . . & . & 136 \\
\hline Arctolimax W... . . & & 30 & Cionella c & collina M. T. & . & 82 \\
\hline Arion albus L. . & & 39 & , & exigua Mke. & . & 82 \\
\hline$" \quad$ ater L. . $\therefore$. & & 38 &, & lubrica Miill. & . & 81 \\
\hline bourguignati Mab. & & 42 & , & nilssoni Malm. & . & 82 \\
\hline brunneus Lehm. . & & 40 & , & nitens Gallenst. & . & 81 \\
\hline cinctus Müll. . . & & 40 & ", & ovata Jeffr. . & . & 81 \\
\hline cinereo-nebulosus $\mathbf{J e}_{\epsilon}$ & & 39 & & viridula Jeffr. & . & 82 \\
\hline citrinus W. . & & 41 & Cleptolin & $\max$ (Malm) . & & 28 \\
\hline empiricorum Fér. & & 39 & Clausilia & æmula W. . & . & 81 \\
\hline flavus Nilss. . & & 41 & , & armata W. . & . & 75 \\
\hline fuscus Müll. • & & 40 & ", & II. & - & 75 \\
\hline hortensis Fér. & & 42 & , & tta Str. & - & 77 \\
\hline lævis $11 \cdot \dot{\text { म }} \cdot$ & & 40 & ", & ta W. . & - & 77 \\
\hline 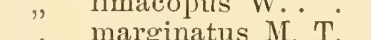 & • & 41 & " & a llont. & - & 60 \\
\hline sedius. & & 3 & " & Stir & & 79 \\
\hline mülleri M. 'T. . & & 39 & $"$ & A. S. & & 81 \\
\hline nilssoni Poll. & & 41 & "' & curta A. S. & & 80 \\
\hline , rufus $\mathrm{L}$. . & & 40 & & dubia Drp. & & 00 \\
\hline Arianta Leach . . & & $\tilde{\partial} 1$ & & elongata A. S. & & 8 \\
\hline Armiger Hn. . . . & & 124 & & erronea $\mathrm{W}$. & & 77 \\
\hline Balea lucifuga B. . . & & 73 & , & exigua $W_{0}$. & . & 77 \\
\hline , perversa $\mathrm{L}_{\text {. }}$. & & 72 & , & extensa W. & . & 80 \\
\hline pirostoma W.. & & 73 & :, & iosa W. . & . & 80 \\
\hline$"$ polita $\mathrm{W}$. . . & & 73 & , & lior A. S. & . & 79 \\
\hline ., rayana $B$. & & 73 & , & gracilis W.. . & . & 76 \\
\hline suecana $\mathrm{IV}$. & & 73 & , & implicata Bz. & . & 75 \\
\hline Bathyomphalus Ag. . . & & 117 & , & labiosa Rm. & . & 80 \\
\hline Buliminus montanus Drp. & & 59 & & laminata Mont. & - & $7 \dot{x}$ \\
\hline ," oblongus W. & & 59 & , & oma W. & . & . \\
\hline ,, obscurus Müll & & 59 & , & nilssoni WV. & . & 76 \\
\hline Bulinus Adans. . . . & & 110 & & obsoleta A. I. & . & 77 \\
\hline Bythinia bottnica $\mathrm{Cl}$. & & 133 & " & ommæ W. . . & - & 78 \\
\hline ., decipiens Mill. & & 134 & ", & oreas W. & . & 80 \\
\hline excavata Jeffr. & & 134 & " & parvula Stud. & . & 76 \\
\hline gemina W. & & 133 & " & personata W. & . & 78 \\
\hline gigas Mörch . & & 13 & $"$ & pirostoma W.. & . & $7 \%$ \\
\hline inflata Hans. & & 13 & & ta Drp. & . & 75 \\
\hline atricata Mill. & & 133 & & plicatula Drp. & . & 80 \\
\hline leachi Sh. . & & 135 & , & plicifera W. & . & 74 \\
\hline michaudi Duv. & & - 134 & , & propinqua W. & - & 74 \\
\hline ma IV. & & $13 \dot{4}$ & & retracta $\mathrm{W}$. & . & 77 \\
\hline producta Mke. & & 133 & ", & rolphi Leach & - & 79 \\
\hline aina Cout. & - & 13 & & scanica W.. & - & 80 \\
\hline W. $\cdot$ & & 134 & & sejuncta W. & & 78 \\
\hline tentaculata L. & & 13 & $"$ & trionalis & & \\
\hline , ventricosa Mke. & & 133 & & $Z .$. & . & 75 \\
\hline Calyculina Cl. . . . . & & 147 & & subrugosa W. & . & 77 \\
\hline Carychium inflatum $\mathrm{Hn}$. & & 198 & $"$ & subspeciosa II. & . & 76 \\
\hline , minimum Müll. & & 90 & " & truncatula WV. & - & 78 \\
\hline Cocilianella aci & & 82 & ., & tumida A. S. . & . & 79 \\
\hline
\end{tabular}


Pag.

Clausilia ventricosa Dxp. . . 79

Clausiliastra Pfr. . . . . . 74

Conulus alderi Gray . . . . 32

" fabricii Bk. . . . 33

, fulvus Miill. . . . 32

, mortoni Jeffr. . . . 32

$\begin{array}{rr}., \quad \text { praticola Reinh. . } & 32 \\ \text { Corneola Cl. . . . } 144\end{array}$

Cusmicia Brus. . . . . 76

Cyclostoma elegans Müll. . . 130

Cyrenastrum B. . . . . . 144

Diplodiscus W. . . . . . 115

Dreissena ampla W. . . . 191

fluviatilis Pall. . 190

locardi (B.) Loc. . 190

occidentalis Loc. . 190

Euanodonta W.

westerlundi Loc. . 190

Eulimax M. T. . . . . 26

Eulota Hn. . . . . . . . 53

Euryomphala IV. . . . . . 50

Fluminina $\mathrm{Cl}$. . . . . . 149

Fossaria W.. . . . 108

Fossarina Cl. . . . . , 151

Gonostoma Held . . . . . 46

Gulnaria Leach . . . . . 95

Gyraulus Ag. . . . . . . 118

Gyrorbis Ag. . . . . . 115

Gyrorbis Fitz. . . . . . 141

Helicella M. T. . . . . 56

Helix aculeata Müill. . . . 45 adela W. . . . . . 45

adspersa Mï̈ll. . . . 58

albolabiata W. . . . 57

alpicola Fér. . . . . 52

anderssoni $\mathrm{Cl}$. . . . 54

arbustorum L. . . . 51

bidens Chemn. . . . 47

colliniana B. . . . 51

concimna Jeffr. . . 48

conica Jeffi. . . . 49

conoidea IV. . . . . 52

conoidea W. . . . 54

conspurcata Drp. . . 55

corasia W... . . 50

costata Müll. . . . 45

crassa $\mathrm{W}$. . . . . 58

creticola Mörch . . 52

creticola Mörch . . . 57

creticola W. . . . 51

depilata C. Pfr. . . . 49

depressa Held . . . 53

efasciata W. . . . . 52

ericetorum Müll. . . 56
Helix foereli B. . . . . . 53

fruticum Mï̈ll. . . . 54

gesneri Hn. . . . 58

gotlandica W. . . . 53

gyrata W. . . . . 48

harpa Say . . . . 46

hispida L. . . . . . 48

hortensis Müll. . . . 57

hybrida Poir . . . 58

hypnicola Mab. . . 53

incarnata Müll. . . . 51

insularum W. . . . 54

intersecta Poir. . . 55

lamellata Jeffr. . . . 45

lapicida L. . . . . . 47

liberta W. . . . . . 49

ludoviciana (Drt.) . . 57

lutescens D. \& M. . . 52

marginata TV. . . 57

maroinella C. G. W. . 58

medelpadensis Cl. . . 47

morboso-albina $\mathrm{Rm} . \quad$. 52

mörchi W. . . . 49

nebulata Mke. . . 48

nemoralis Müil. . . . 55

nilssoniana Bk. . . 56

obvia Hn. . . . . . 56

obvoluta Miill. . . 46

olandica W. . . . 53

pallida W. . . . . . 52

perforata W. . . . 58

picea Rm. . . . . 52

pomatia L. . . . . 58

porrecta W. . . . . 57

pulchella Müll. . . . 45

rubiginosa A. S. . . 50

rudis $\mathrm{Rm}$. . . . . . 53

schrenki Midd. . . 54

septemtrionalis $\mathrm{Cl}$. . $\quad 48$

septemtrionalis $\mathrm{Cl}$. . 52

sepulcrorum W. . . 49

striata. Müll. . . . . 5a

strigella Drp. . . . . 51

subglobosa WI. . . . 51

sublævis IV. . . . . 49

tardigyra W. . . . . 46

thamnivaga Mab. . . 53

trochoidalis Roff. . . 52

tumescens W.. . . 50

umbrosa C. Pfr. . . . 47

unifasciata Poir. . . 55

Hippeutis Ag. . . . . . 125

Hyalinic alliaria Mill. . . 36

anceps TW. . . . 36 
$23 \pm$ C. A. Westerlund, Synopsis Molluscorum Extramarinor. Scandin.

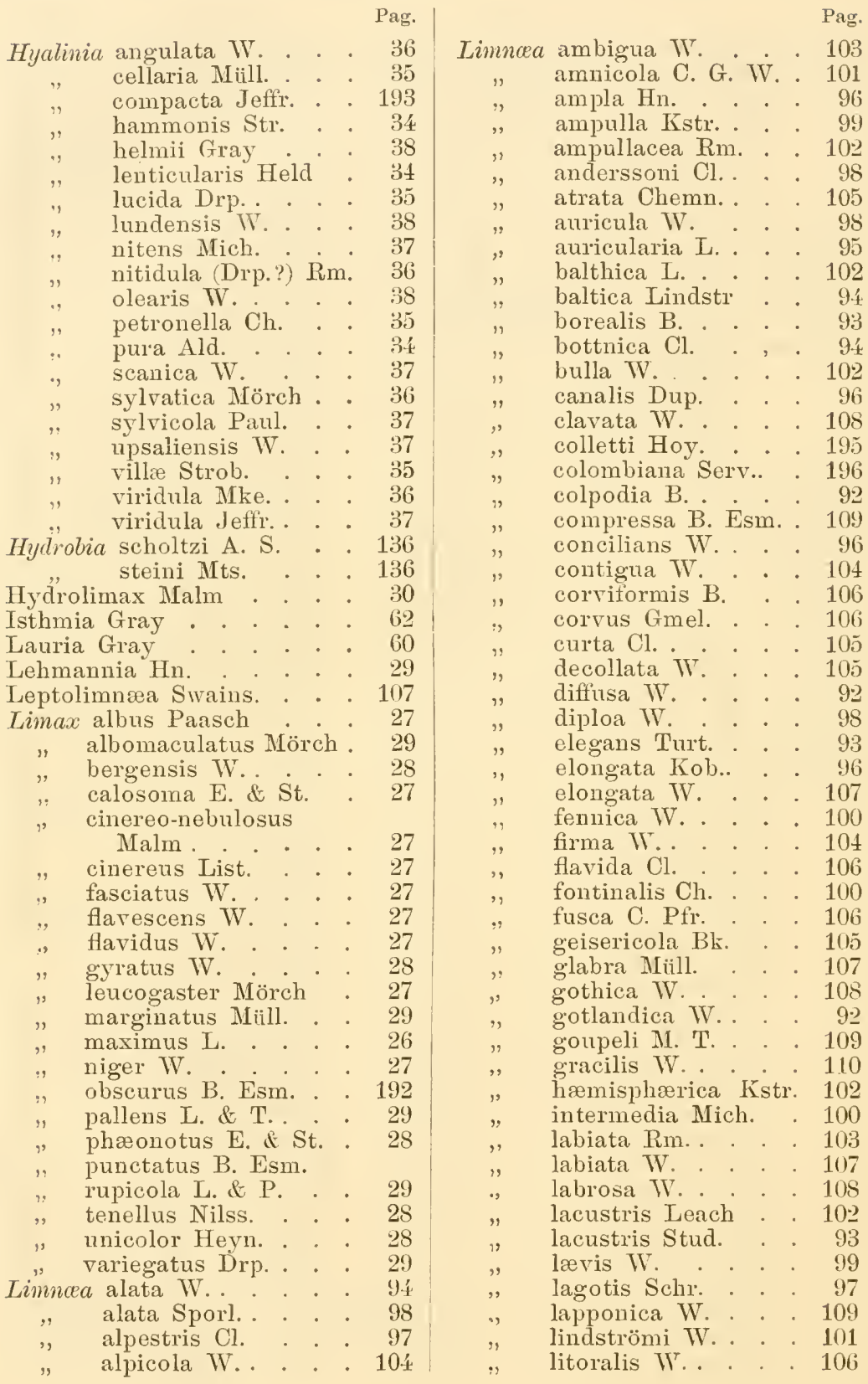


Pag.

Limncea livonica Rob. . major M. T. margaritacea M. T. maritima Jeffr. maximella Colb. media Hu. meta $W^{\top}$. microstoma Baud. minima Colb. monnardi $\mathrm{Hn}$. mucronata Held mörchi W. neréni WV. nordmanni TV. oblonga Put. obtusa Kob. orthoplenra IV. ovata Drp. paludinoides Mörch palustris Müll. patula Da C. peregra Müill. peregro-ovata Kob. petronia W. plicata $\mathrm{W}$. prævaleus W. . prisea IV. producta Colb. pumila Mörch raboti W. retusa W. roseo-labiata Sturm. schneideri B. Esm. septemtrionalis $\mathrm{Cl}$. . sericina C. G. TV. sophronia $\mathrm{W}$.

stagnalis L. . steenstrupi Cl. stenostoma WT. subinflata $\mathrm{WV}$ subulata W.

succinea Nilss. surcula WV. torsa W.

truncatula M̈̈ill. turgida Mke turricula Held. ventricosa $\mathrm{Hn}$. westerlundi Loc. . Limnophysa Fitz.

Luceva Oken

Lymnus Montf.

Malacolimax Malm Margaritacea borealis $\dot{W}$.
95

108

98

104

109

94

104

109

110

97

97

108

92

94

109

101

105

99

103

105

102

103

195

10.4

97

103

99

91

97

101

92

93

109

196

100

92

91

103

107

101

91

103

100

93

108

93

107

96

92

105

88

91

28

167 $\begin{array}{ccc}\text { Margaritacea compressa W. . } & 167 \\ \text { elongata (Lam.?) } & \\ \text { Nilss. . . } & 167 \\ \text { margaritifera L. } & 166 \\ \text { ". } & \text { roissyi Mich. } & \mathbf{1 6 7} \\ \text { ventricosa W. . } & \mathbf{1 6 7}\end{array}$

Monacha Hn. . . . . . . 51

Nauta Leach . . . . . . 111

Neritina baltica Bk. . . . . 143

. fluviatilis L. . . . 142

,fontinalis Brard . . 142

" lacustris L. . . . 142

, litoralis L. . . . 1.13

" spirata W. . . . 142

Neritostoma Klein . . . . 83

Paludina contecta Mill. . . 130

." gallandi B. . . . 131

lacustris Bk. . . . 131

isseli B. . . . . 132

" occidentalis B. . 131

", paludosa B. . . . 131

"vivipara L. . . . 131

Papillifera Hn. . . . . . 75

Patula globosa Friedl. . . 43

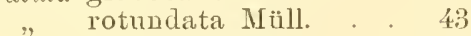

" ruderata Stud. . . 44

" rupestris (Stud.) . . 44

". striatella Ant. . . . 44

" turtoni Flem. . . 43

Petasia M. T. . . . . 47

Physa achatina Gm. . . . 110

", albescens Mörch . . 110

" bulla Müll. . . . . . 110

" fontinalis L. . . . . 110

" grandis TV. . . . . 111

". hypnormm L. . . . 111

" oblonga TV. . . . 111

" perissiana Dup. . . . 112

" semiglobosa W. . . 111

Pirostoma Vest. . . . . . 79

Pisidium acuminatum Cl. . 154

., amnicum Mäll. . . 149

amnicum Müll. . . 149
arcticum WV. . . 155

cinereum Ald. . . 154

elongatum Baud. . 150

esmarkianum $\mathrm{Cl}$. $15 \mathrm{~S}$

fedderseni IV. . . 153

feröense Mörch . 159

flavescens Cl. . . 154

fontinale Pfr. . . . 153

fragile Cl. . . . 158

glaciale $\mathrm{W}$. . . . 149

globulare Cl. . . 150

henslowianum Sh. 151 


\begin{tabular}{|c|c|c|c|c|c|}
\hline & & Pag. & & & Pag. \\
\hline Pisidium 1 & hoyeri Cl. . & 153 & Planorbis & dispar $\mathrm{IT}_{\text {. . }}$ & 118 \\
\hline,$\quad 1$ & lapponicum Cl. . & 156 & , & draparnaldi Sh.. & 122 \\
\hline " & lilljeborgi Cl. . & 153 & , & dubius Hn. . & 115 \\
\hline ", & lindströmi Cl. & 153 & , & ecarinata IV. & 114 \\
\hline , & lovéni Cl. & 154 & " & elophilus B. . & 112 \\
\hline$\because$ & maguificum Cl. . & 158 & , & euphæus B. & 125 \\
\hline " & malmi Cl. $\cdot$ & 150 & $"$ & filocinctus W. & 114 \\
\hline , & martensi Cl. & 152 & ", & fontanus Lightf. & 20 \\
\hline , & milium Held . & 106 & $"$ & frigidus WV. . & \\
\hline$"$ & nitidum Jen. & 102 & $"$ & gelidus W. & 124 \\
\hline$"$. & oidaleum IV. & 157 & " & $\begin{array}{l}\text { glaber Jeffr. . } \\
\text { goösi IY }\end{array}$ & 122 \\
\hline$\ddot{~}$ & pallidum Jeffi. & 154 & $"$ & gothicus & 116 \\
\hline , & parvulum Cl. . & 151 & $"$ & gredleri Bz. & 123 \\
\hline$"$ & personatum Malm & . 158 & $"$ & hispidus Drp. : & 121 \\
\hline , & poulseni Cl. . . & 156 & $"$ & imbricatus MIüll. & 124 \\
\hline .. & pulchellum Jen. . & . 152 & , & labiata W. . & 114 \\
\hline$"$, & pusillum Jen. . & 158 & $"$ & labiata WV. & 117 \\
\hline. & scholtzi Cl. . & 156 & , & leptostoma $\mathrm{W}$. . & 126 \\
\hline , & solidum Cl. . & 158 &, & limophilus W. . & $12 \cdot 2$ \\
\hline., & sphenoides W. . & 156 & $"$ & leucostoma Mill. & 117 \\
\hline , & striolatum Baud. & 150 & $"$ & nautileus L. . . & 124 \\
\hline , & subtruncatum Malm & 155 & $\cdot$, & nitidus Müll. . . & 126 \\
\hline ", & suecicum Cl. . . & 151 &, & nummularis Mörch & 114 \\
\hline$"$ & supinum A. S. & 150 & , & oelandicus W. . & 127 \\
\hline " & transversale $\mathrm{Cl}$. & 153 & " & perizii Dup. . & 117 \\
\hline & unioides W. . & 157 & , & poulseni TV. & 116 \\
\hline Planorbis & alatus W. & 119 & $"$ & riparius WV. . & 126 \\
\hline , & albus Müll. . & 119 & $"$ & socius W. & 120 \\
\hline$"$ & ammonoceras WV. & 112 & $"$ & spirorbis I. & 117 \\
\hline$"$ & angigyrus W. . & 121 & , & spurius W. & 119 \\
\hline , & arcticus Bk. . & 120 & $"$ & strömi W. & 120 \\
\hline$"$ & borealis (Lov.) W. & 121 & " & turgidus $W$. . & 115 \\
\hline$"$ & carinatus Mïll. . & 114 & , & umbilicatus Mü̈ll. & 113 \\
\hline ; & catina W... & 114 & ,. & vortex L. . & 115 \\
\hline , & ens Held & 116 & & vorticulus Trosch. & 116 \\
\hline$"$ & chordus IV. & 196 & Pomatia & Bk. . . & 58 \\
\hline$"$ & cinctutus W. & 119 & Pseudan & odonta B. . & 188 \\
\hline " & clessini W. & 127 & Punctum & pygmæum Drp. & 43 \\
\hline$"$ & cochlearis W. & 124 & Pupa alp & bestris Ald. . . & 66 \\
\hline$"$ & complanatus L. & 125 & " anc & conostoma Lowe & 60 \\
\hline , & compressus Morch & 115 & ans & gustior Jeftr. . & 71 \\
\hline$"$ & concinnizs IV. & $\cdot$ & ant & tivertigo Drp. . & 68 \\
\hline " & $\begin{array}{l}\text { contortus L. } \\
\text { correctus W. }\end{array}$ & $\begin{array}{l}118 \\
. \quad 123\end{array}$ & arc & ctica Wallenb. & 60 \\
\hline$"$ & cormetis L. & . 112 & ave & enacea Bruo: & · \\
\hline 2 & crista L. . . & 124 & bif & fida IV. & 70 \\
\hline & cryptomphalus W. & 113 & big & rranata Rm. & 61 \\
\hline & i Mörch & 117 & cal & llista W. . & $\dot{.}$ \\
\hline & deflexus W. & 121 & cel & lata W. & 64 \\
\hline & difformis W. & 118 & cle & vei W. & 71 \\
\hline , & disciformis Jeffr. & 115 & col & llina W. . . & 71 \\
\hline$"$ & discoides Reinh. & 115 & $\mathrm{col}$ & lumella Bens. & 62 \\
\hline$"$ & discus (Parr.) Rm. & 116 & $\cos$ & stulata Nilss. & 62 \\
\hline
\end{tabular}


Pag.

Pupa cylindracea Da C. . . 60 dalecarlica W. . . 61 daliaca TV. . . . . . 64 dicra W. . . . . 64 edentula Drp. . . . . 62 elongata $\mathrm{Cl} . \quad . \quad . \quad .61$ eremia IV. . . . . 67 erlandi W. . . . . . 194 extima H. . . . . . 66 ferox IV. . . . . . 69 gemma W. . . . . 65 genesii Gr. . . . . . 64 globula W . . . . 68 gothorum W. . . . 72 gravida $W$. . . . . 69 inermis W. . . . . 60 inornata Mich. . . . 62 lilljeborgi W. . . . . 68 lundströmi W. . . . 61 merita W. . . . . 68 minutissima $\mathrm{Hn}$. . . 63 mitis W. . . . . 66 monas W. . . . 70 montigena $W$. . . 60 moulinsiana Dup. . . 69 muscorum Müll. . . 61 octodentata Stud. . . (i9 odontostoma IV. . . 63 otostoma IV. . . . . 72 ovoidea XV. . . . 65 paucidens IV. . . . 61 pineticola W... . 65 producta IV. . . . . 71 pusilla Müll. . . . 70 pygmæa Drp. . . . . 69 quadridens IV. . . $\quad 69$ ronnebyensis IV. . . 67 seminulum WV. . . . 69 septemtrionalis W. . 67 sexdentata Mout. . . 69 shuttleworthiana Ch. . 67 subcereana W. . . . 61 sublordeum W. . . 61 substriata Jeffr. . . 70 tumida W. . . . . . 71 turritella W. . . . . 62 unidentata C. Pfx. . 61 Pupilla Leach . . . . . . 61 Rivulina Cl. . . . . . 150 Segmentina Flem. . . . . 126 Sphreriastrum B. . . . . . 1 t3 Spharium brochonianum B. 148 corneum C. . . . $144^{4}$ danicum Cl. . . 148
Sphcerium duplicatum Cl. 1.16 firmum $\mathrm{Cl}$. . . $14 \dot{4}$ lacustre Müll. . . 147 mamillanum TV. . 145 mamillare Gass. . 147 nucleus Stud. . $14 t$ orcula W. . . . 14 . ovale Fér. . . . 14.5 physale TV. . . 147 pilacre $\mathrm{WV}$. . . 147 rivicola (Leach)

Lam. . . . 143 ryckholti Norm. . 148 steini A. S. . . 148 strictum (Norm.) Cl. 1is subsolidum Cl. . $14 x$ suecicum WV. . . 146 tomentosum IV. . 146 ulienyi WT. . . 145 westerlundi Cl. $\quad 146$

Sphyradium Leach . . . 61

Spirodiscus Stein . . . . 112

Striatella W. . . . . . . 55

Succinea agonostoma Kstr. . 88 altaica Mts. . . 88 arenaria Bouch. . . 89 borealis W. . . 89 brevispirata Band. . 85 charpentieri D. \& M. $\quad 83$ charpyi Baud. . . S' contorta IV. . . . 87 contortula Baud. . 87 costellata W. . . 86 delavali W. . . . 88 diphya W. . . . 86 drouetia M. T. . 84 elata Baud. . . . 87 elongata $\mathrm{Hn}$. . . 85 ferussina M. T. . . $8: 3$ gigantea B. . . . 83 gracilis Ald. . . . 87 groenlandica Bk. . 85 impura Hn. . . . s8 impura $W^{\top} . . . \quad . \quad 89$ lenta W. . . . . 87 limnoidea Pic. . . 83 lutetiana Mab. . 88 mediolanensis Villa Si nilssoniana $\mathrm{Cl}$. . . $\quad 86$ norvegica IV. . . 88 oblonga Drp. . . . 88 olivula Baud. . . . 194 parvula Pasc. . . 84 pfeifferi Rm. . . . 8.5 


\begin{tabular}{|c|c|c|c|c|c|c|}
\hline & & & & & & ag. \\
\hline & nea propinqua Baud & & 86 & Unio tumidus Retz. & & 162 \\
\hline " & putris L. & & 83 & Vallonia Risso . . . & & $4 \dot{x}$ \\
\hline & radiata $\mathrm{C}$. G. II & & 81 & Valvata alpestris (Blaun.) & & 139 \\
\hline & sarsi B. Esm. . & . & 194 & " ambigua TT. & & 138 \\
\hline . & stagnalis Gass. & & 194 & antiqua Sow. . & . & 136 \\
\hline & subelegans $\mathrm{TT}$. & & 87 & borealis Milach. & & 138 \\
\hline & subglobosa Pasc & & Sú & cristata Müll. . & . & 41 \\
\hline & temporalis $\mathrm{W}$. & & 84 & cyclomphala $\mathrm{W}$. & & \\
\hline & trianfracta Da C & & 84 & discors IV. . & . & 138 \\
\hline & rentricosa Pic. & & 86 & fennica $\Pi^{\top}$. . & . & 197 \\
\hline Tacl & ea Leach . . . . & . & 56 & frigida TI. . & . & 141 \\
\hline Core & uilia (Faure-Big.) St & Stud. & 60 & glacialis WW. & . & 139 \\
\hline Tric & ia Hn: . . . . . & . & . & halopea $\mathrm{TT}$. & . & 139 \\
\hline Trol & idina Ad. . . . & . & 140 & lilljeborgi TI. . & . & 137 \\
\hline Trot & idiscus Stein & . & 113 & malleata $\mathrm{W}$ & & 140 \\
\hline Unio & acutus Rm. . & . & 160 & macrostoma Steel & nb. & $1 \div 0$ \\
\hline., & ater Nilss. & & & & . . & 140 \\
\hline ., & barys $\Pi$ & & 16 & piscinalis Müll. & . & 137 \\
\hline , & batavus M. \& R. & . & 16 & pulchella TV. . & . & 140 \\
\hline , & borythenicus Serr. & . & . & lla Müll. & . & 137 \\
\hline .. & ITS Mörch & . & . & ti 1 . & . & 139 \\
\hline , & conus Spengl. . . & . & . & umbilicata $\mathbb{T}^{r}$. & . & 140 \\
\hline$\because$ & crassus Retz. & . & . & Velletia Gray. . . . & . & 128 \\
\hline , & noræ B. . & . & 16 & Vertigo Müllı. . & . & 70 \\
\hline .. & delphinus Spengl. & . & 10 & Titrea contracta W. . & . & 3 \\
\hline .. & deshayesi Mich. . & . & 16 & , $\quad$ crșstallina Müll. & . & \\
\hline & WI... & . & 11 & mea B. . & . & 33 \\
\hline & stris Rm. . & ${ }^{\circ}$ & 163 & Titrina angelicæ Bk. . & . & 31 \\
\hline & limicola Mörch & . & 1( & ensis Tlicn. & & \\
\hline ,. & limosus Nilss. . & . & . & ì Jeffr. . & . & 31 \\
\hline , & mülleri Rm. . & . & . & holmiensis W. . & . & $3:-$ \\
\hline , & oralis Mont. & - & . & pellucida Müill. & . & \\
\hline & s Bk. . & . & 10 & perforata $W$. & . & 31 \\
\hline & atus Lam. & $\cdot$ & 16 & Serophila Held. & . & $5 t$ \\
\hline & s Mke. . & & 10 & Zonitoides borealis $\mathrm{Cl}$. & & 34 \\
\hline & saccatus Rm. . & & 10 & ,, nitidus Müill. & & 33 \\
\hline & septemtrionalis (B.) & & 16 & norvegicus B. E & Esr & \\
\hline ,. & subdecurvatus $\mathrm{TV}$. & $\cdot$ & 16 & "parisiacus Mab. & & \\
\hline$\cdot$ & tumidulus Loc. & & . $16{ }^{\prime}$ & Zua Leach . . . . . & & \\
\hline
\end{tabular}





